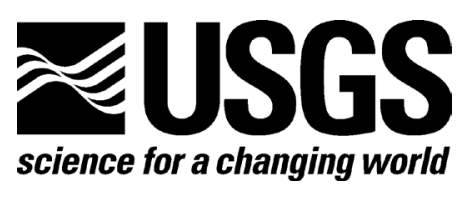

\title{
HAWAIIAN VOLCANO OBSERVATORY 1983 Annual Administrative Report
}

INTRODUCTORY NOTE BY THOMAS L. WRIGHT AND JENNIFER S. NAKATA

COMPILED BY JENNIFER S. NAKATA

\author{
SUMMARY 83 \\ SEISMIC DATA, JANUARY TO DECEMBER 1983 \\ By JENNIFER S. NAKATA, Alvin H. TOMORI, \\ Robert Y. KoyAnagi, AND Wilfred R. TANigaWA \\ CHRONOLOGICAL SUMMARY \\ BY ROBERT W. DECKER
}

OPEN-FILE REPORT 2007-1343

U.S. DEPARTMENT OF THE INTERIOR

U.S. GEOLOGICAL SURVEY 


\section{U.S. Department of the Interior DIRK KEMPTHORNE, Secretary \\ U.S. Geological Survey Mark D. Myers, Director}

\section{U.S. Geological Survey, Reston, Virginia 2007}

For product and ordering information:

World Wide Web: http://www.usgs.gov/pubprod

Telephone: 1-888-ASK-USGS

For more information on the USGS - the Federal source for science about the Earth, its natural and living resources, natural hazards, and the environment: World Wide Web: http://www.usgs.gov

Telephone: 1-888-ASK-USGS

Any use of trade, product, or firm names is for descriptive purposes only and does not imply endorsement by the U.S. Government.

Although this report is in the public domain, permission must be secured from the individual copyright owners to reproduce any copyrighted material contained within this report. 


\section{INTRODUCTORY NOTE}

The Hawaiian Volcano Observatory Summaries have been published in the current format since 1956. The Quarterly Summaries (1956 through 1973) and the Annual Summaries (1974 through 1985) were originally published as Administrative Reports. These reports have been compiled and published as U.S. Geological Survey Open-File Reports. The quarterly reports have been combined and published as one annual summary. All the summaries from 1956 to the present are now available as .pdf files at http://www.usgs.gov/pubprod.

The earthquake summary data are presented as a listing of origin time, depth, magnitude, and other location parameters. Network instrumentation, field station sites, and location algorithms are described. Tilt and other deformation data are included until Summary 77, January to December 1977. From 1978, the seismic and deformation data are published separately, due to differing schedules of data reduction.

There are eight quarters - from the fourth quarter of 1959 to the third quarter of 1961 — that were never published. Two of these ( $4^{\text {th }}$ quarter $1959,1^{\text {st }}$ quarter 1960) have now been published, using handwritten notes of Jerry Eaton (HVO seismologist at the time) and his colleagues. The seismic records for the remaining six summaries went back to California in 1961 with Jerry Eaton. Other responsibilities intervened, and the seismic summaries were never prepared.

\section{Chronology}

The following Kìlauea eruption chronology covers the two recent reports and the six missing quarters:

\begin{tabular}{|l|l|l|l|}
\hline Location & Beginning Date & Ending Date & Comment \\
\hline Kìlauea Iki crater (Kîlauea's summit) & $11 / 14 / 1959$ & $12 / 20 / 1959$ & 19 eruptive episodes \\
\hline Kapoho (lower east rift zone) & $1 / 13 / 1960$ & $2 / 18 / 1960$ & 4 eruption stages \\
\hline Halemaumau (Kīlauea's summit) & $2 / 24 / 1961$ & $2 / 24 / 1961$ & $\begin{array}{l}\text { Intermittent activity during } \\
\text { uninterrupted inflation fol- } \\
\text { lowing the 1960 eruption }\end{array}$ \\
\hline Halemaumau (Kīlauea's summit) & $3 / 22 / 1961$ & $3 / 25 / 1961$ & Same as above. \\
\hline Halemaumau (Kīlauea's summit) & $7 / 10 / 1961$ & $7 / 17 / 1961$ & Same as above. \\
\hline Heiheiahulu (middle east rift zone) & $9 / 22 / 1961$ & $9 / 25 / 1961$ & $\begin{array}{l}\text { First historical east rift erup- } \\
\text { tion at this location }\end{array}$ \\
\hline
\end{tabular}

The 1959-1960 eruptions were among two of the most spectacular Kilauea eruptions. The HVO staff was kept busy with acquisition of unusually high quantities of instrumental data and observations of the two sequences, which were separated by less than one month. Even with a year's interval before the beginning of the summit-east rift sequence in 1961, the staff never caught up, and the seismic records were set aside for later study.

A total of 1,672 earthquakes-1,106 for 1960 and 566 for 1961-are part of HVO's cataloged database. The annual listings have been appended to the $1^{\text {st }}$ Quarter Report of 1960 and to the $4^{\text {th }}$ Quarter Report for 1961 . The number of earthquakes is probably low, biased toward the larger magnitudes. The entire HVO catalog, including 1960 and 1961, is accessible from the ANSS CATALOG SEARCH site at http://www.ncedc.org/anss/catalog-search. 
UNITED STATES DEPARTMENT OF THE INTERIOR

GEOLOGICAL SURVEY

HAWAIIAN VOLCANO OBSERVATORY

SUMMARY 83

SEISMIC DATA, JANUARY TO DECEMBER 1983

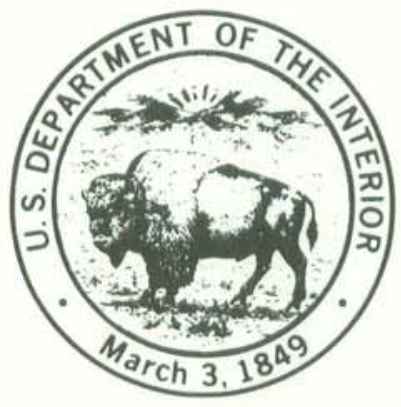

This report (map) is preliminary and has not been reviewed for conformity with U.S.Geological Survey editorial standards (and stratigraphic nomenclature).

Any use of trade names is for descriptive purposes only and does not imply endorsement by the U.S.G.S.

Menlo Park, California 1984 


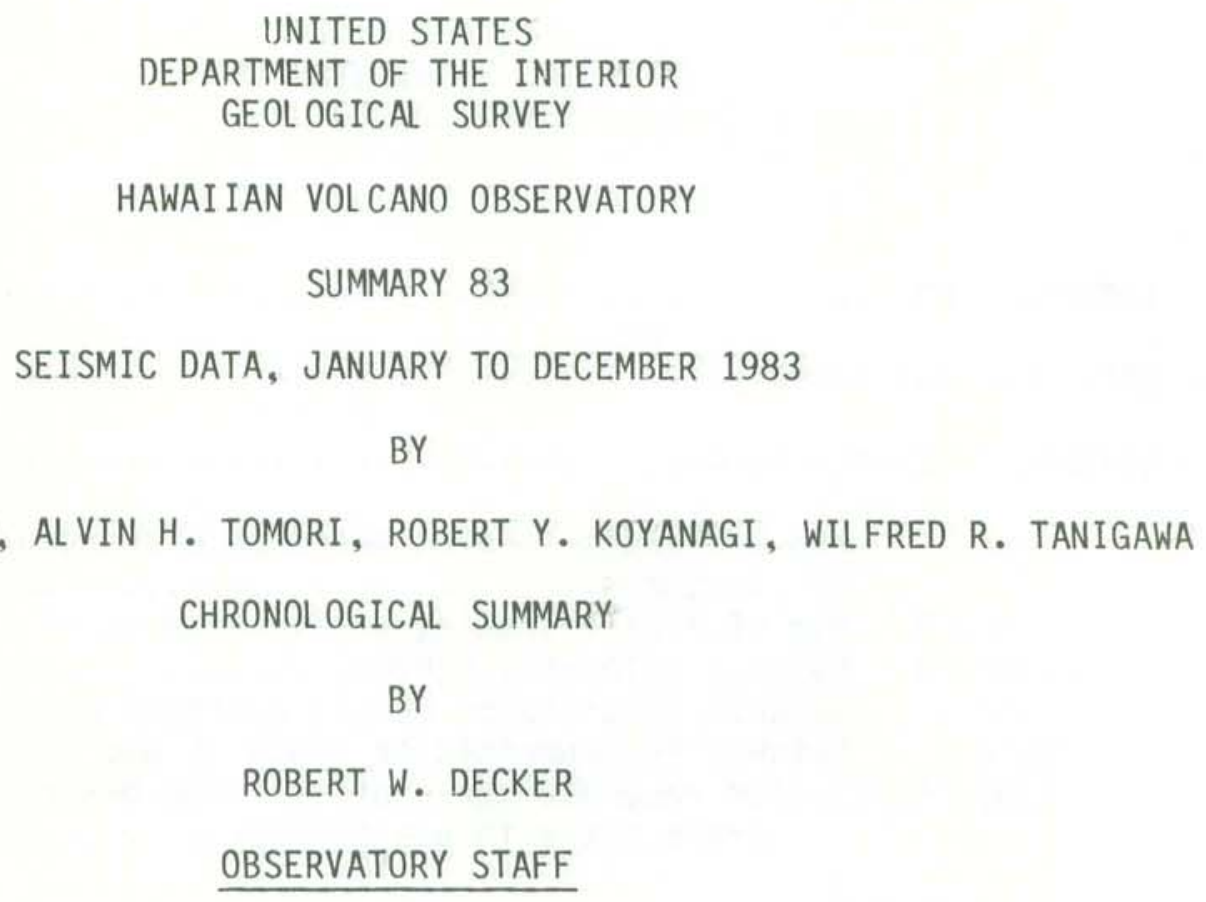
CHRONOL OGICAL SUMMARY

JENNIFER S. NAKATA, ALVIN H. TOMORI, ROBERT Y. KOYANAGI, WILFRED R. TANIGAWA OBSERVATORY STAFF

GEOL OGY

TONI D. ABADIE

NORMAN G. BANKS

CHRISTINA A. NEAL

EDWARD W. WOL FE

GEOPHYSICS

CRAIG C. CRISSINGER

ROBERT W. DECKER (SCIENTIST-IN-CHARGE

JOHN J. DVORAK

KENNETH T. HONMA

GARY Y. HONZAKI

DALLAS B. JACKSON

GEORGE KOJIMA

ROBERT Y. KOYANAGI

ALL AN J. L ARGO

JENNIFER S. NAKATA

ARNOL D T. OKAMURA

GARY S. PUNIWAI

WILFRED R. TANIGAWA

AL VIN H. TOMORI
GEOCHEMISTRY

L. PAUL GREENL AND

REGINAL D T. OKAMURA

(CHIEF OF OPERATIONS)
SUPPORT

THOMAS T. ENGL ISH DOROTHY C. FOOTER JOHN C. FORBES J.D. GRIGGS RONAL D Y. HANATANI

MARIAN M. KAGIMOTO

ROBERT A. RATHBURN

MAURICE K. SAKO

J. B. STOKES

PAUL INE N. TAMURA

AKIRA YAMAMOTO

KENNETH M. YAMASHITA

JOAN M. YOSHIOKA

AFFIL IATES

JANE BUCHANAN-BANKS (GEOL OGIST)

JOHN P. LOCKWOOD (GEOL OGIST)

RICHARD B. MOORE (GEOL OGIST)
MARJORIE SUMMERS

VICKI B. TAYL OR

FRANK A. TRUSDELL L 


\section{CONTENTS}

Page

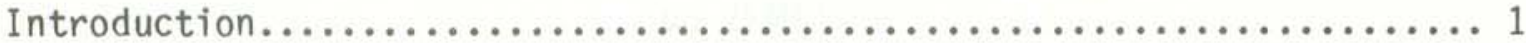

Chronological Summary........................................

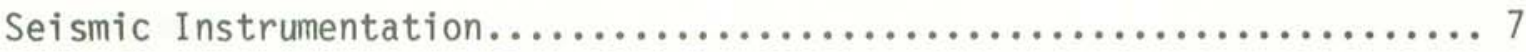

Figure 1 Map of Hawaii showing geographic and geologic

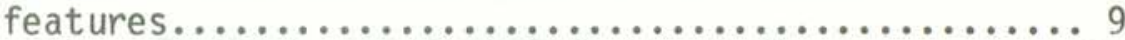

Figure 2 Map of Hawaii showing active seismic stations............10

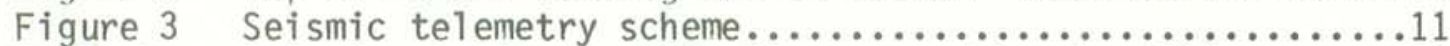

Table 1 Seismic stations on Hawaij operated by the USGS........12

Table 2 Seismic instrumentation types in use by HVO............13

Figure 4 System response curve of the four basic seismograph types in use by HVO..................14

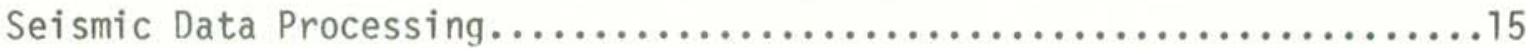

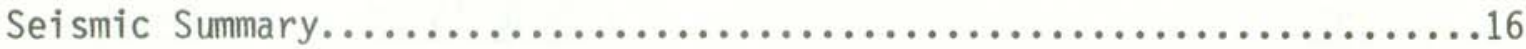

Table 3 Number of earthquakes and minutes of tremor recorded on seismographs around Kilauea...........16

Table 4 Coordinates of named regions used for classifying

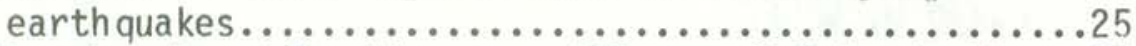

Fiqure 5 Earthquake classification map, Kilauea, $0-5 \mathrm{~km}$ depth...27

Figure 6 Earthquake classification map, Kilauea, 5-13 km depth...28

Figure 7 Earthquake classification map, Hawaii, 0-13 km depth...29

Figure 8 Earthquake classification map, Hawaii, 13-99 km depth...30

Figure 9 Epicenter map of Kilauea, depths $0-5 \mathrm{~km}, \mathrm{M}>2 \ldots \ldots \ldots . .31$

Figure 10 Epicenter map of Kilauea, depths $5-13 \mathrm{~km}, M^{-}>2 \ldots \ldots \ldots 32$

Figure 11 Epicenter map of the Mauna Loa region, depths

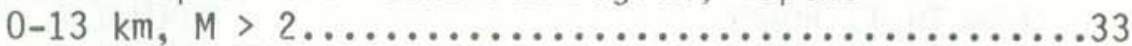

Figure 12 Epicenter map of Hawaii, depths $13-60 \mathrm{~km}, M>2 \ldots \ldots \ldots 34$

Figure 13 Epicenter map of Hawaii, depths $0-13 \mathrm{~km}, M>3 \ldots \ldots \ldots . .35$

Figure 14 Epicenter map of Kilauea caldera, depths $0-5 \mathrm{~km}$,

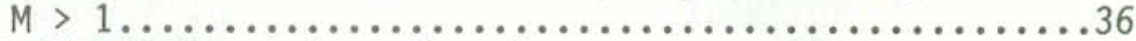

Table 5 List of all located earthquakes.........................

Table 6 List of located earthquakes of magnitude 3.0 or

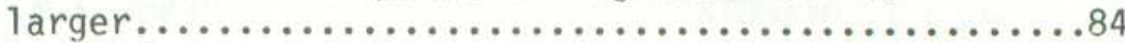




\section{INTRODUCTION}

The Hawaiian Volcano Observatory (HVO) summary presents data gathered during the year together with a chronological narrative describing the volcanic events and significant observatory related activities. The seismic summary is offered without interpretation as a source of preliminary data. The seismic summary is complete in the sense that all data routinely gathered by the observatory are included. The emphasis in collection of tilt and deformation data has shifted from quarterly measurements at a few water-tube tilt stations ("wet" tilt) to a larger number of continuously recordina borehole tiltmeters, repeated measurements at numerous spirit-level tilt stations ("dry" tilt), and surveying of level and trilateration networks. Because of the large quantity of deformation data now gathered and differing schedules of data reduction, the seismic and deformation summaries will be published separately.

The HVO summaries have been published in various formats since 1956 . Summaries prior to 1974 were issued quarterly, but cost, convenience of preparation and distribution, and the large quantities of data dictated an annual format beginning with summary 74 for the year 1974. Summary 74 includes an extensive description of the seismic instrumentation, calibration and processing used in recent years. The present summary includes enough background information on the seismic network and processing to use the data and understand the essentials of how it was gathered.

A report tabulating the instrumentation, calibration and recording history of each seismic station in the network by Klein and Koyanagi is available as a USGS open file report ("Hawaiian Volcano Observatory Seismic Network History 1950-79," U.S.G.S. open file report 80-302, 1980). It is designed as a reference for users of seismograms and phase data, and so includes and expands the information in the station table in this summary. 


\title{
CHRONOL OGICAL SUMMARY - 1983
}

by

\author{
Robert W. Decker
}

1983 was a busy year for both Hawaiian volcanic and seismic activity. The middle-east-rift eruption of Kilauea which began on January 3 continued intermittently throughout the year with 12 major phases of high-volume lava emission. Mauna Loa showed signs of unrest in terms of increased seismic activity and changing patterns of surface deformation, and the observatory was rocked and damaged by intensity VIII to IX shaking from the magnitude 6.6 Kaoiki earthquake 12 kilometers beneath the southeast flank of Mauna Loa on November 16.

The middle-east-rift eruption of Kilauea was preceded by 24 hours of high seismicity, harmonic tremor and rapid summit deflation as the feeding dike of the eventual eruption was emplaced beneath a $10 \mathrm{~km}$ segment of the east rift zone from about $1 \mathrm{~km}$ west of Makaopuhi Crater to the vicinity of Pu'u Kamoamoa. Over the next 4 to 5 days the dike extended another $6 \mathrm{~km}$ downrift to about $2 \mathrm{~km}$ east of Kalalua. The eruptive outbreak occurred on the north rim of Napau Crater and moved eastward along a line of fissures for 8 kilometers. The volume of lava erupted in the first phase was approximately 11 million $\mathrm{m}^{3}$, but the amount of summit deflation ( 60 to 70 million $\mathrm{m}^{3}$ from leveling data) indicates that at least 50 million $\mathrm{m}^{3}$ of magma moved from the summit into the newly formed dike system.

Subsequent phases of the eruption occurred from the same dike system without new seismic swarms, indicating that the continuing eruption was using portions of the same conduit system established during the first phase. Phases 2 through 12 were remarkably similar in period, volume and summit tilt changes, suggesting a repetitive process controlled by some interaction of summit magma reservoir pressure and the gas content and pressure head of the lava column in the erupting dike. The major lava emission of phases 2 through 12 ocurred on the following dates: (2) February 25-March 4; (3) March 28-April 9; (4) June 13-17; (5) June 29-July 3; (6) July 22-25; (7) Auqust 15-17; (8) September 6-7; (9) September 15-17; (10) October 5-7; (11) November 6-7; and (12) November 30December 1. The average time period between the main onsets of the major phases was 30 days; the shortest was 9 days, and the 1 ongest was 77 days. In general, the phases were of shorter duration as the eruption progressed. However, the volumes of the phases, with one exception, were remarkably similar; the average was $11.9 \times 10^{6} \mathrm{~m}^{3}$, the largest (phase 3 ) was $25.6 \times 10^{6} \mathrm{~m}^{3}$, and the smallest (phase 8) was $7.6 \times 10^{6} \mathrm{~m}^{3}$. This combination of shorter eruption duration but similar eruption volume indicates that later phases (excluding phase 1) were erupted at higher eruption rates. This conclusion is supported by field estimates of eruption rates, and by the general increase in the rate of summit subsidence and harmonic tremor measured during each subsequent phase. 
Table I. The middle-east-rift eruption of Kilauea, 1983 (Ed Wolfe).

\begin{tabular}{|c|c|c|c|}
\hline Phase & $\begin{array}{l}\text { Total Area } \\
\text { Covered } \\
\left(\times 10^{6} \mathrm{~m}^{2}\right) \\
\end{array}$ & $\begin{array}{c}\text { Total Volume } \\
\text { of Lava } \\
\left(x 10^{6} \mathrm{~m}^{3}\right) \\
\end{array}$ & $\begin{array}{l}\text { Duration and Average Production Rate } \\
\text { (total volume/duration) }\end{array}$ \\
\hline 1 & 4.8 & 11.0 & 96 hours; $104,000 \mathrm{~m}^{3} /$ hour \\
\hline 2 & 2.7 & 11.5 & $\begin{array}{r}\text { lava shield: } 360 \mathrm{hrs} ; 1400 \mathrm{~m}^{3} / \mathrm{hour} \\
\text { cone: } 181 \mathrm{hrs} ; 60,000 \mathrm{~m}^{3} / \mathrm{hr}\end{array}$ \\
\hline 3 & 7.9 & 25.6 & 288 hours; $90,000 \mathrm{~m}^{3} /$ hour \\
\hline 4 & 2.2 & 10.9 & 100 hours; $110,000 \mathrm{~m}^{3} /$ hour \\
\hline 5 & 3.4 & 12.9 & 90 hours; $143,000 \mathrm{~m}^{3} /$ hour \\
\hline 6 & 2.8 & 9.4 & 73 hours; $120,000 \mathrm{~m}^{3} /$ hour \\
\hline 7 & 3.7 & 10.3 & 57 hours; $180,000 \mathrm{~m}^{3} /$ hour \\
\hline 8 & 2.0 & 7.6 & 24 hours; $315,000 \mathrm{~m}^{3} /$ hour \\
\hline 9 & 2.1 & 8.6 & 50 hours; $173,000 \mathrm{~m}^{3} /$ hour \\
\hline 10 & 2.7 & 14.0 & 60 hours; $232,000 \mathrm{~m}^{3} /$ hour \\
\hline 11 & 4.3 & 12.1 & 43 hours; $281,000 \mathrm{~m}^{3} /$ hour \\
\hline 12 & 3.0 & 8.1 & 35 hours; $231,000 \mathrm{~m}^{3} /$ hour \\
\hline OTAL S-1983 & 26.0 & 141.8 & 1457 hours $=61$ days \\
\hline
\end{tabular}

A11 12 phases of the 1983 eruption have a total volume of about $140 \times 10^{6}$ $\mathrm{m}^{3}$ and covered about $26 \mathrm{~km}^{2}$ of land surface. (In Table I, the total area covered is less than the sum of the areas covered in each phase $--41.6 \mathrm{~km}^{2}$; this difference represents later phases resurfacing areas covered in earlier phases.) Most of the area covered by the new flows was dense ohia-fern forest, but some was barren flows from eruptions during the 1960's, and some was in a relatively undeveloped land subdivision called Royal Gardens. Phases 2 through 5 produced flows that entered Royal Gardens, destroying about 15 dwellings and covering about 330 empty house lots and their access roads. Flows from phases 6 through 12 came from the same vent area as phases 4 and 5; however, the main lava channel in these later phases developed on the northeast rather than the southeast side of the growing cinder and spatter cone. For this reason most of the flow volume in phases 6-12 stayed on the north side of the rift zone in the ohia-fern jungle. Phases 2 and 3 built a small lava shield capped by an $80 \mathrm{~m}$ high spatter and cinder cone at their principle vent area just south of Pu'u Kahauale'a. Phases 4 through 12 built another 80 m-high spatter and cinder cone just inside the National Park boundary about $1.5 \mathrm{~km}$ uprift from $\mathrm{Pu}^{\prime} u$ Kahauale'a. 
The erupted lavas before phase 5 were relatively low in magnesium content and the associated magmatic gases were low in carbon dioxide and high in sulfur dioxide. These data and the generally low eruption temperatures $\left(1120^{\circ}-1130^{\circ} \mathrm{C}\right)$ indicate that much of the erupted magma prior to July 1983 was stored at shallow levels in the summit or middle east rift zone for months to years prior to eruption. Even after phase 5 the $\mathrm{C} / \mathrm{S}$ ratio of the gas remained low. Nevertheless, the total gas content was great enough to produce lava fountains during all of the major eruptive phases, reaching a maximum height of $300 \mathrm{~m}$ in phases 3 and 10 .

Mauna Loa began to show signs of increasing unrest with a swarm of microearthquakes beneath its summit region in early March 1983. Earthquake counts increased to a maximum of 400 to 500 per day compared to a former background of 2 to 20 events per day. Deformation measurements indicated that the summit was continuing to inflate and that the center of the uplift had shifted to the east. Small earthquake swarms in June and July were followed by a major swarm in September that peaked at about 700 events per day. Following that swarm the number of microearthquakes per day beneath Mauna Loa decreased back to almost average levels by the end of 1983. The general similarity in the pattern of Mauna Loa earthquakes and deformation in 1983 compared to 1974 -- the year prior to the last eruption of Mauna Loa in July 1975 -- prompted HVO to issue a statement indicating a higher-than-average probability of an eruption of Mauna Loa during 1984.

During 1983 there were 35 earthquakes beneath the Hawaij region that equalled or exceeded magnitude 4, two that exceeded magnitude 5, and one that exceeded magnitude 6 . The magnitude 5.1 earthquake occurred $9 \mathrm{~km}$ beneath the south flank of Kilauea on September 9. It was apparently another one of the larger earthquakes associated with the seaward displacement of the south flank of Kilauea.

On November 16, 1983, at 06:13 (HST) a magnitude 6.6 earthquake occurred at $12 \mathrm{~km}$ depth in the Kaoiki seismic zone beneath the southeast flank of Mauna Loa. The epicenter was slightly south of the mid-point of a line drawn between the summit calderas of Kilauea and Mauna Loa. The summit area of Kilauea was subjected to intensity VIII to IX shaking which did considerable damage but fortunately caused only a few minor injuries. Sectors of the crater rim road near Waldron Ledge fell into the caldera, and heavy damage was caused to roads where they cross or closely parallel the outer caldera escarpments of Kilauea. Considerable structural damage also occurred in Hilo. The total damage estimate was about $\$ 7,000,000$.

First-motion studies indicate a source mechanism of nearly east-west compression and north-south extension. A northeast-southwest, right-lateral strike-slip fault is the apparent fracture direction from both surface breaks on the Mauna Loa strip road and aftershock sequences of smaller Kaoiki earthquakes. The initial location of hundreds of aftershocks from this magnitude 6.6 earthquake suggest multiple fractures on conjugate slip planes allowing east-west contraction and north-south extension. The stress field that caused this large earthquake is interpreted to originate from the summit dilation of Mauna Loa and Kilauea as magma accumulates in their subsurface reservoir systems. Thus, although the earthquakes is a tectonic earthquake in the sense that it represents shear failure from major compressive stresses, it can also be considered volcanic in origin because the stresses are generated by magmatic 
pressures. As in much research, sharp distinctions between classes and types of phenomena become more blurred as investigations progress.

The automatic earthquake location microprocessor designed by Rex Allen and Jim Ellis of the USGS in Menlo Park, CA, did a creditable job of locating in nearly real time the earthquake swarm that preceded and accompanied phase 1 of the $1983 \mathrm{Kilauea}$ eruption. This allowed field observers to be in the remote area near Napau Crater at the time and location the eruption began.

Deformation studies again provided some interesting new results. The emplacement of the dike that initiated the 1983 eruption was associated with a nearly simultaneous increase in earthquakes and strain within the south flank of Kilauea. The coastline moved about 1 meter seaward in response to a 2 to 3 meter dilation of the rift zone. The difference between these displacements represents compression of the south flank of Kilauea between the middle east rift and the seacoast. The increase in south flank earthquakes apparently resulted from this increase in compressive stress from the injection of a new dike $12 \mathrm{~km}$ long reaching from a $3 \mathrm{~km}$ depth to the suface. Recording tiltmeters along the track of the intruding dike indicated a downrift migration rate of $1.9 \mathrm{~km} / \mathrm{hr}$ in comparison to the downrift migration rate of the earthquakes of $0.7 \mathrm{~km} / \mathrm{hr}$. This suggests that the dike broke upward from a pre-existing molten core along the rift zone below $3 \mathrm{~km}$ following the injection of a pulse of new magma into the middle-east-rift pipeline.

The amount of summit subsidence and elevation of the eruption vents of Kilauea's east-rift-zone eruptions show a strong correlation indicating magmastatic pressure control of the volume of magma that moves from the summit into the rift zone. Calculations of the relationship between radial summit tilt measured at HVD (Uwekahuna), and the change in elevation of the apex of the summit bulge of $\mathrm{Kilauea,} \mathrm{the} \mathrm{volume} \mathrm{change} \mathrm{of} \mathrm{the} \mathrm{surface} \mathrm{bulge,} \mathrm{and} \mathrm{change} \mathrm{in}$ the pressure in the shallow magma reservoir beneath the summit are approximately: 1 microradian of $S 60 \mathrm{E}$ tilt (deflation) $=5.5 \mathrm{~mm}$ subsidence $=330,000$ $\mathrm{m}^{3}$ decrease in volume $=0.9$ bars pressure decrease.

Sulfur dioxide gas from Halemaumau has increased in volume from about 150 tons per day in 1982 to about 300 tons per day in 1983. The ratio of carbon to surfur measured at Halemaumau is 17; the ratio at the 1983 eruption site on the middle east rift is 0.2 . This dramatic loss of carbon dioxide appears to take place as magma moves into and is stored within the shallow magma reservoir system beneath the summit of Kilauea.

Self-potential (S-P) anomalies measured along the east rift of Kilauea have added some interesting insights into the 1983 eruption. An increase in S-P at Kalalua, downrift from the eruptive vents, supports seismic data which indicate that the intrusive dike on January 7, 1983, moved farther downrift than the eruptive vents. In addition, S-P increases at Escape Road near Mauna U1u between October and November, 198?, suggest that new magma was moving into the upper east rift 2 months prior to the beginning of the 1983 eruption.

The apparent presence of iridium in fume from the 1983 eruption reported by $W$. H. Zoller et al in Science (December 9, 1983, p. 1118-1121) has added fuel to the not debate about interpretation of the iridium anomaly at the Cretaceous-Tertiary boundary. 
The observatory staff consists of 8 scientist and 16 support personnel. In addition 18 students and volunteers helped HVO during 1983. The staff monitored 48 seismic stations, 1204 electronic distance measurement lines, $275 \mathrm{~km}$ of level lines, 99 tilt stations, 15 recording tiltmeters, 13 strain gauges, 130 gravity stations, 12 self-potential lines, 1 controlled-source electromagnetic induction 10op with 4 receiver stations, 21 gas-sampling and temperature sites, and many special devices to monitor the long-lasting eruption such as time-lapse cameras, video camera, trip wires, and portable tremormonitoring seismometers. Many of HVO's monitors record continuously; others are observed at various intervals. The remote location of the eruption site required over 500 hours of helicopter support which posed serious problems of safety, expense, communication and logistics. The data reduction, graphics, and data storage of the overall monitoring program are largely handled by HVO's computer system.

Four USGS geologists continued their mapping projects on Hawaii. Their close affiliation with HVO has been essential during the 12 vigorous eruption phases that required 24 hour per day surveillance.

Four members of the combined HVO staff and geological affiliates were involved in investigations of Indonesian volcanoes, Pagan Volcano in the Marianas, Rabaul Volcano in Papua New Guinea, and of Mount St. Helens and Yellows tone.

HVO cooperated with 60 guest investigators during the year for time periods up to 2 months. Staff members and guest investigators presented 26 papers at scientific meetings and published 34 papers and 4 open-file reports. HVO also issued weekly, monthly and annual reports, as well as daily updates during the major eruptive phases.

Hawaii Volcanoes National Park estimated they had 2,800,000 visitors in 1983. About $50 \%$ of these visitors stopped at HVO to view Kilauea Caldera and to look in HVO's windows at the seismographs and the eruption chronology board. About 1600 visitors including 22 student groups, 4 professional-meeting groups and George Ariyoshi, Governor of Hawaii, toured the inner workings of the observatory. A new record to TV and news photographers was established with an estimated 300 visiting the observatory. Many of these were multiple visits throughout the year by the same news teams.

It was exhausting, but a great year at HVO. 


\section{SEISMIC INSTRUMENTATION}

The network. The Hawaiian Volcano observatory maintains an extensive telemetering seismometer network on the island of Hawaii. In 1983 the seismometer network consisted of 51 stations; two are low-gain multicomponent stations (optical), nine are three-component, and 40 are vertical only. The coverage is most complete on and around Kilauea Volcano. With the exception of self-contained systems at Uwekahuna and Hilo stations, all seismometer signals from the short period network are telemetered to the observatory for recording.

Figure 1 is a map of selected geographic and geologic features, Figure 2 shows the seismic stations which were operated on the Island of Hawaii during the year, and Figure 3 indicates the telemetry scheme for the respective seismic stations. Table 1 lists all seismic stations operated by the U.S. Geological Survey in Hawaii during 1983. Listed are station names, three- and four-letter codes, coordinates in degrees and minutes, elevation in meters, and other data described below. In addition to seismometers listed in Table 1, a horizontal seismometer of Type 3 or $\mathrm{H} 1$ and a long-period, three-component set of Press-Ewing seismometers are operated in the Uwekahuna vault, all recorded on photographic paper.

Instrumentation and recording. Each telemetering station has a voltage controlled oscillator (VCO) for FM multiplex transmission to HVO via either hardwire or radio. These telemetering stations are now all of Type 1, the OEVE standard system used in USGS seismic networks (see Table 2 for details). After discrimination, the analog signals from 36 stations are recorded on two Develocorders using $16 \mathrm{~mm}$ microfilm. FM signals from the telemetering network are also recorded directly on one-inch magnetic tape. Selected larger events are copied onto condensed FM library tapes which are currently archived in Men10 Park and archived in digital form at HVO as part of the routine location processing. The type of recording used for each station (in addition to magnetic tape for the telemetered stations) is coded in Table 1 as follows: D - Develocorder film, S - smoked paper drum, P - photographic paper, H - Helicorder paper.

In addition to the standard stations, optical seismographs are maintained at Uwekahuna (HVO), Hilo, Maui, and on Oahu (Honolulu station operated by the Pacific Tsunami Warning Center). The less sensitive optical records are used primarily for amplitude measurements for magnitude calculations to supplement readings from the high-gain stations. The paper (optical) records as well as the $16 \mathrm{~mm}$ Develocorder microfilm are archived at HVO.

In late August, 1982, Rex Allen and Jim Ellis from the U.S. Geological Survey's Office of Earthquake Studies came to HVO to install their seismic monitoring system uniquely designed to automate picking of P-phase onset times, measuring coda lengths, and determinining preliminary epicenters for adequately recorded earthquakes. The Hawaii picker is configured to monitor a maximum of 80 stations, but is currently wired to 45 stations. Attached to the picker are a Datasouth Printer and an Apple II microcomputer. The picker output is logged on a disk and printed out on chart paper within three minutes after the earthquake occurs. The data collected on disk is tranferred to the Eclipse, the main computer system, on a daily basis using a second Apple II. The earthquake records are filtered and run through our standard location program (HYPOINVERSE). Summary listings and epicenter plots are prepared on chart paper, and the phase data are archived on tape. The picker output is routinely 
compared with readings from our standard format. Modifications are being made to adapt the automated system to better accommodate seismic monitoring needs around Hawaii's active volcanoes.

Seismograph response and calibration. Displacement response curves for the four short-period seismograph types in use are given in Figure 3 . Types three and four are electro-mechanical systems recorded on paper records. The Type 1 curve gives the displacement magnification of the standard OEVE system from ground motion at the seismometer to the seismic trace as seen on a $20 x$ Develocorder film viewer. The curves plot the unit response which should be multiplied by a constant but known factor (CAL, Table 1) to get the response for an individual station. Individual CAL factors for Type 1 seismographs are equal to the peak-to-peak amplitude measured in $\mathrm{mm}$ on the 20X Develocorder viewer of a 10 microvolt $5 \mathrm{~Hz}$ signal introduced to the preamp/VCO in place of the geophone. Calibration is normally done each time a station is visited. CAL factors range from about 1 to 3 , averaging about 4. A detailed history of CAL factors and other data is given in F.W. K1ein and R.Y. Koyanagi, Hawaiian Volcano Observatory Seismic Network History 1950-79, U.S. Geological Survey Open File Report 80-302, 1980. 


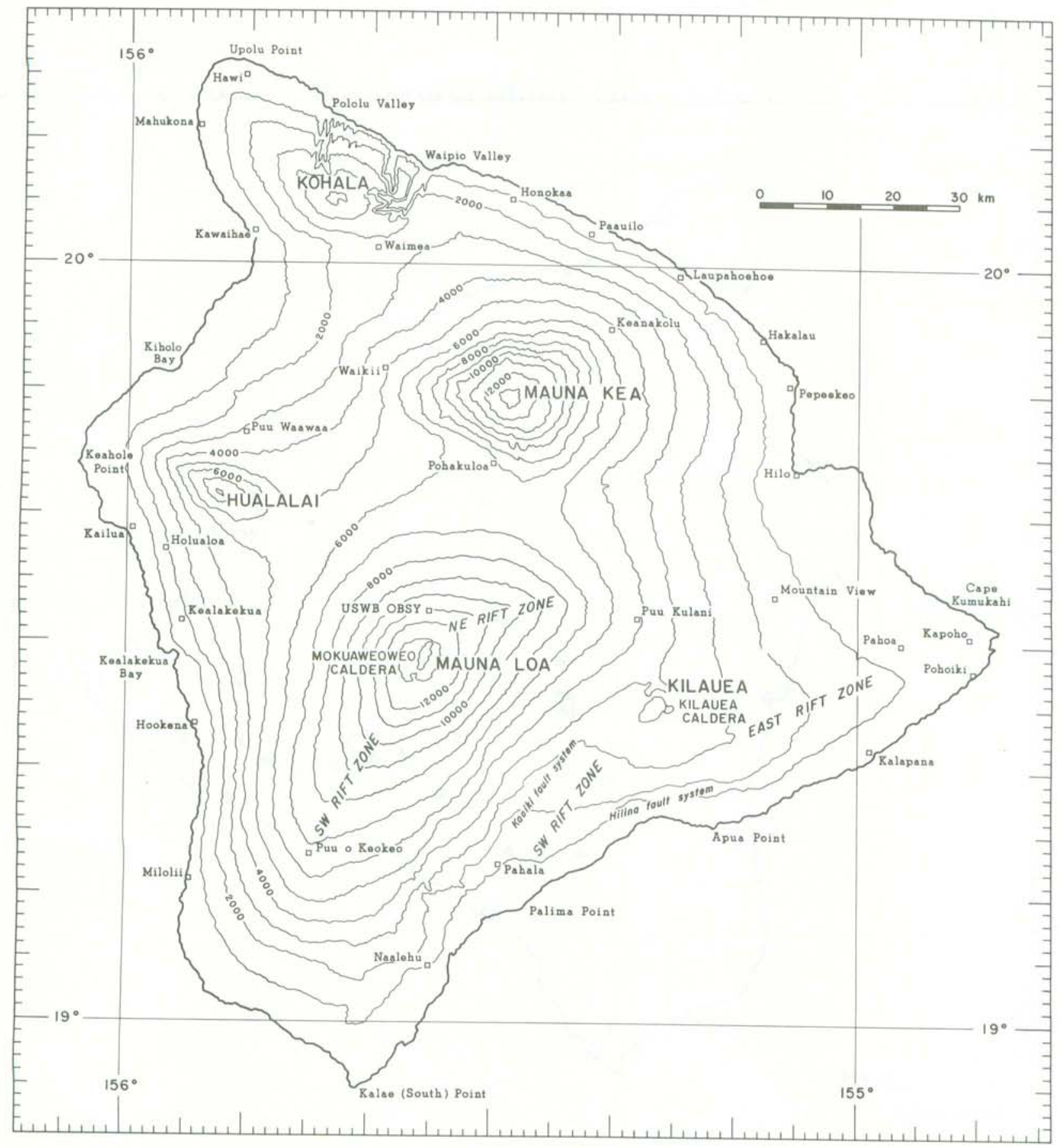

Figure 1. Map of the island of Hawaii showing principal settlements and selected geographic and geologic features. 


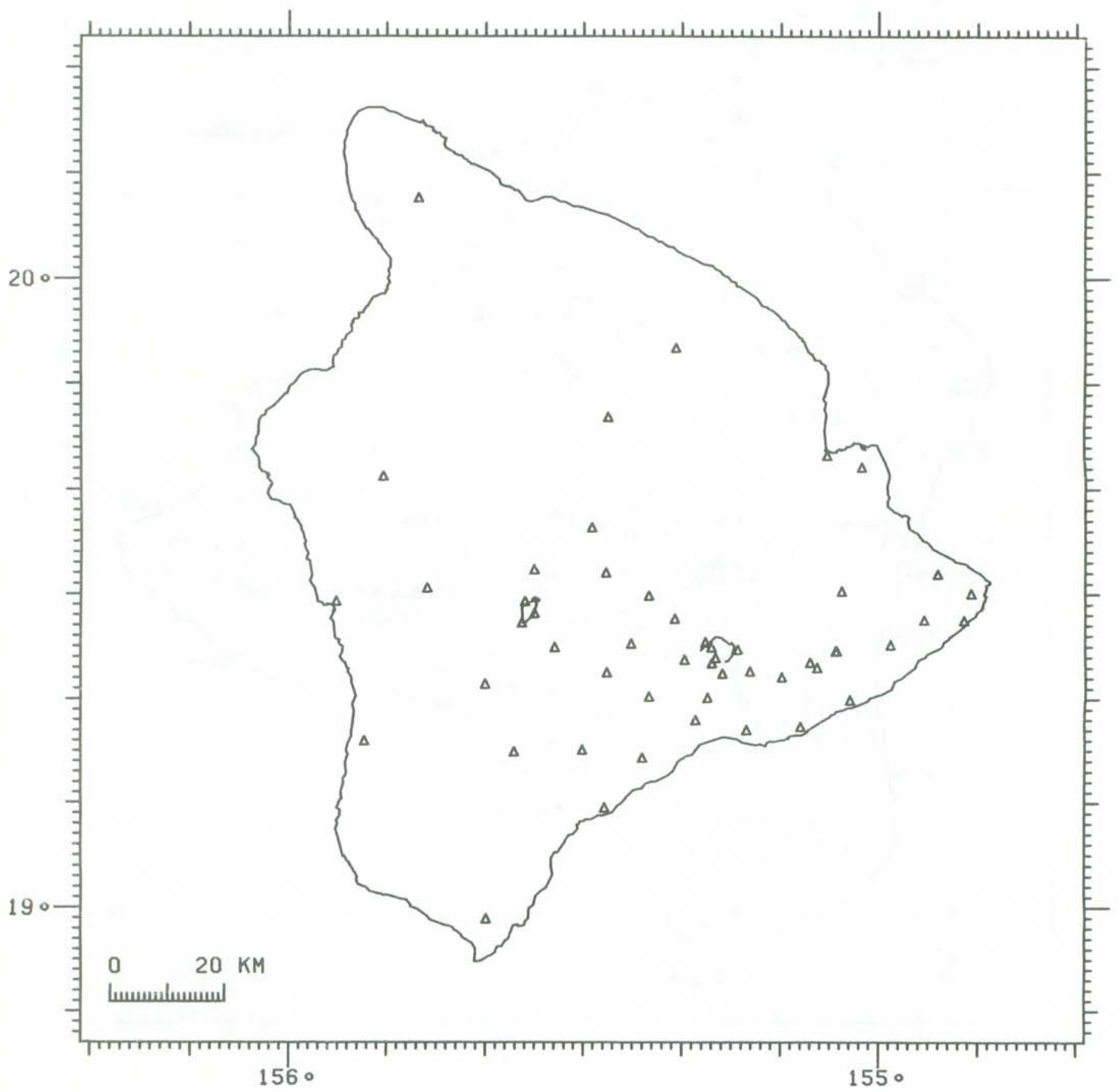

Figure 2. Map of the island of Hawaii showing seismic stations operational during 1983. 


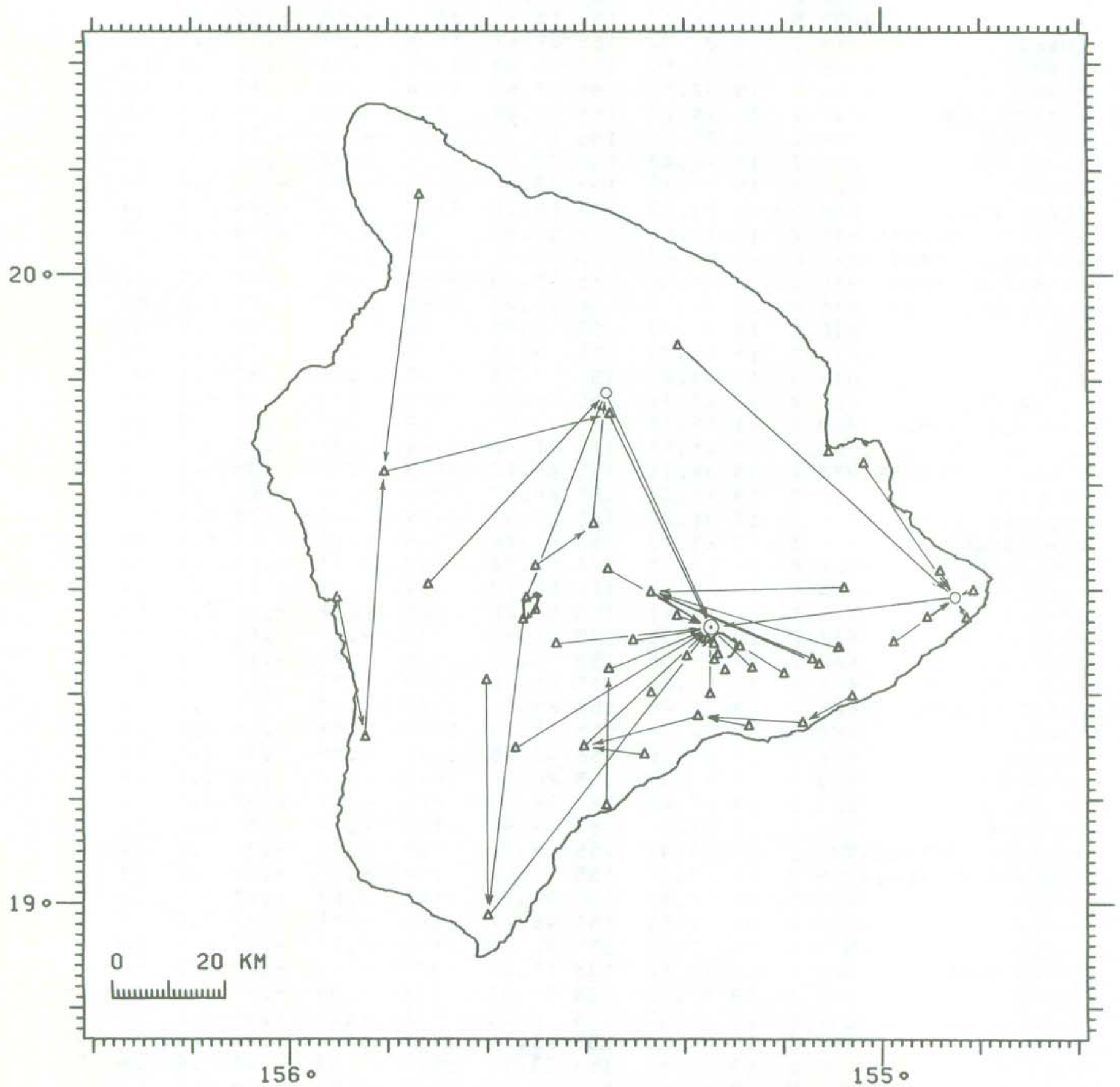

Figure 3. Map of the island of Hawaii showing the telemetry scheme for the Hawaijan Volcano Observatory seismic network.
Legend
$\triangle$ Seismometer location
○ Repeater Station 
Table 1. Selsmometer stations in Hawall operated by the U.S. Geological survey, 1983.

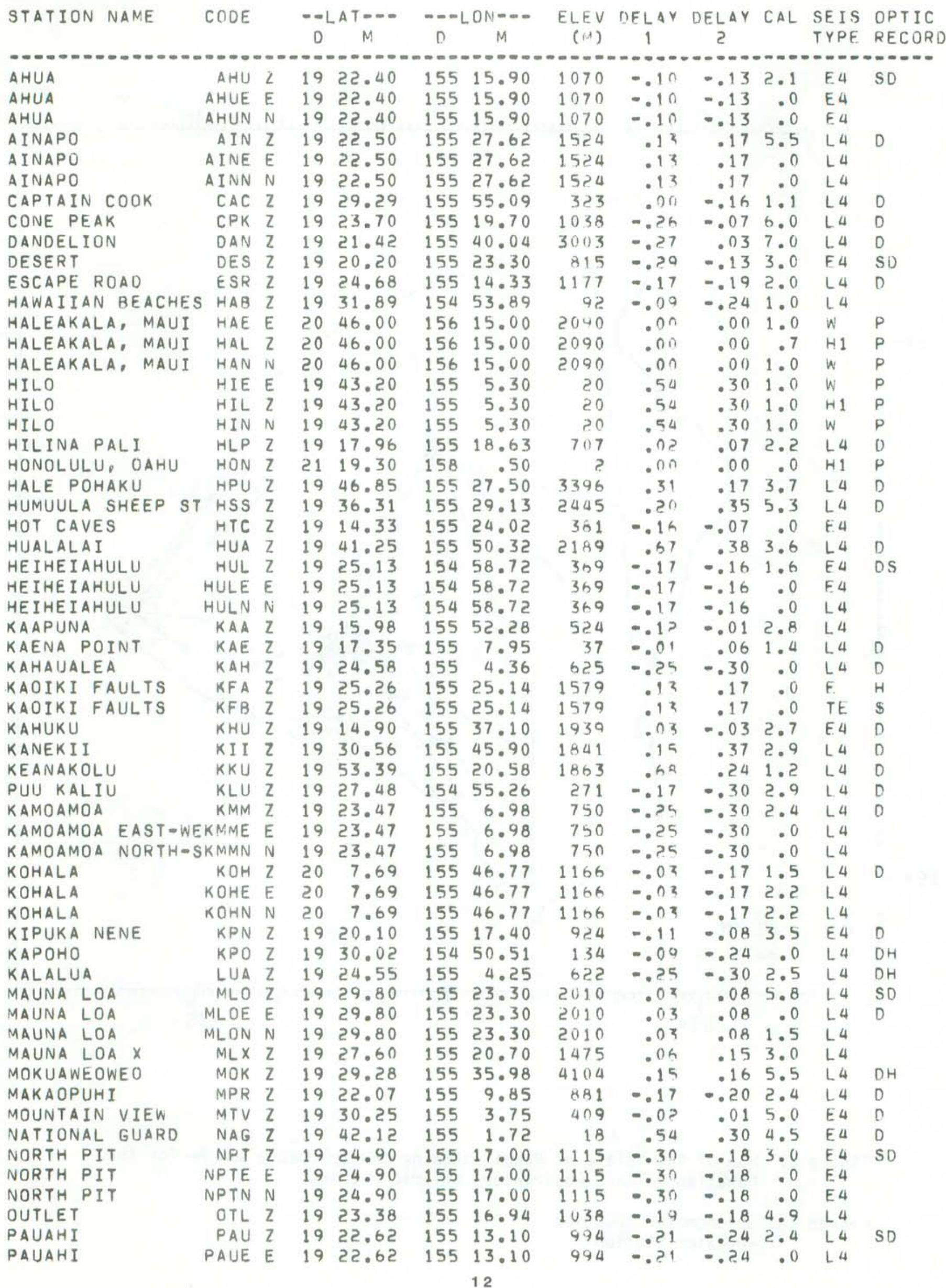




\begin{tabular}{|c|c|c|c|c|c|c|c|c|c|c|c|}
\hline PAUAHI & PAUN & $\mathrm{N}$ & 19 & 22.62 & 155 & 13.10 & 994 &.$- ? 1$ & -.24 & .0 & L4 \\
\hline PUU ULAULA & PLA & 2 & 19 & 32.00 & 155 & 27.67 & $249 ?$ & -.02 & .13 & 5.4 & L \\
\hline POHOIKI & POI & Z & 19 & 27.42 & 154 & 51.22 & 16 & -.00 & $=.24$ & .0 & L 4 \\
\hline POLIOKEAWE PALI & $P O L$ & $z$ & 19 & 17.02 & 155 & 13.47 & 159 & $-.0 ?$ & .03 & 2.8 & E4 \\
\hline PUU PILI & $P P L$ & Z & 19 & 9.50 & 155 & 27.87 & 35 & $\because .15$ & .15 & 1.4 & E4 \\
\hline PUU KAMOAMOA & PUK & 2 & 19 & 23.00 & 155 & 6.25 & 704 & -.25 & -.30 & .9 & L 4 \\
\hline RIM & RIM & $z$ & 19 & 23.90 & 155 & 16.60 & 1128 & -.21 & -.13 & .0 & L 4 \\
\hline SOUTH POINT & SPT & $z$ & 18 & 58.91 & 155 & 39.92 & 244 & -.17 & -.2? & 2.8 & L 4 \\
\hline SOUTH POINT & SPTE & E & 18 & 58.91 & 155 & 39.92 & 244 & -.17 &. .22 & .0 & 1.4 \\
\hline SOUTH POINT & SPTN & $N$ & 18 & 58.91 & 155 & 39.92 & 244 & -.17 & -.22 & .0 & L. 4 \\
\hline SOUTHWEST RIFT & SWR & $z$ & 19 & 27.26 & 155 & 36.30 & 4048 & .01 & .04 & 5.6 & E4 \\
\hline TRAIL & TRA & $z$ & 19 & 24.91 & 155 & 32.96 & 3207 & .00 & .00 & .0 & L4 \\
\hline UWEKAHUNA & UEE & $E$ & 19 & 25.40 & 155 & 17.60 & 1240 & -.21 & .00 & 1.5 & $E$ \\
\hline UWEKAHUNA & UEN & $\mathrm{N}$ & 19 & 25.40 & 155 & 17.60 & 1240 & -.21 & .00 & 1.5 & E \\
\hline UWEKAHUNA & UEZ Z & $z$ & 19 & 25.40 & 155 & 17.60 & 1240 & -.21 & .00 & 1.5 & E \\
\hline UWEKAHUNA & USE & $E$ & 19 & 25.40 & 15 & 17.60 & 1240 & -.21 & .00 & 1.0 & $s$ \\
\hline UWEKAHUNA & USZ & Z & 19 & 25.40 & 15 & 17.60 & 1240 & -.ટ1 & .00 & 1.0 & S \\
\hline WAHAULA & WHA & $z$ & 19 & 19.90 & 15 & 2.92 & 29 & -.10 & $=.04$ & 1.5 & E. 4 \\
\hline WILKES CAMP & WIL & $z$ & 19 & 28.15 & & 35.02 & 4037 & $.2 ?$ & .17 & 2.6 & E4 \\
\hline WEATHER OBSERVAT & WOB & Z & 19 & 32.31 & 155 & 35.01 & 3396 & .00 & .00 & .0 & E4 \\
\hline WOOD VALLEY & wo0 & $\mathbf{Z}$ & 19 & 15.08 & 155 & 30.12 & 909 & -.15 & .06 & 4.6 & E4 \\
\hline
\end{tabular}

Table 2. Seismic Instrumentation Types

The codes in parentheses refer to the seismometer types listed in Table 1.

Type 1. (Codes E, L, and 4) Consists of:

a) Geophone - Electrotech EV-17 (E) or Mark Products L4C (L) $1.0 \mathrm{sec}$. period moving magnet vertical component seismometer or horizontal component adjusted for an output of 0.5 volts/ $\mathrm{cm} / \mathrm{sec}$. and 0.8 critically damped.

b) Preamp/VCO - USGS/OEVE Model J302 or J402 (4) voltage controlled oscillator. Three db points for bandpass filter at $0.1 \mathrm{~Hz}$ and $30 \mathrm{~Hz}$. Signals are transmitted on audio FM carrier over cable or FM radio link to HVO.

Type 3. (Code H1) Consists of:

Electrotech EV-17 or observatory-built $0.8 \mathrm{sec}$. period moving coil seismometer with HVO-built solid state seismic preamplifier, galvanometer driver, and $2 \mathrm{~Hz}$ galvanometer. Peak magnification approximately 40,000 at $4 \mathrm{~Hz}$.

Type 4. (Code S) Consists of:

Sprengnether short period vertical and horizontal seismometers (E-W) wi th 1.5 sec. galvanometers, coupling factor $=0.25,2 x$ critically damped. Peak magnification approximately $1500 \mathrm{x}$ at $2 \mathrm{~Hz}$.

Type 2 instruments have been discontinued.

Code $(W)$ is a Wood-Anderson torsion seismograph.

Codes (TE) and (S5) are experimental seismometers. 


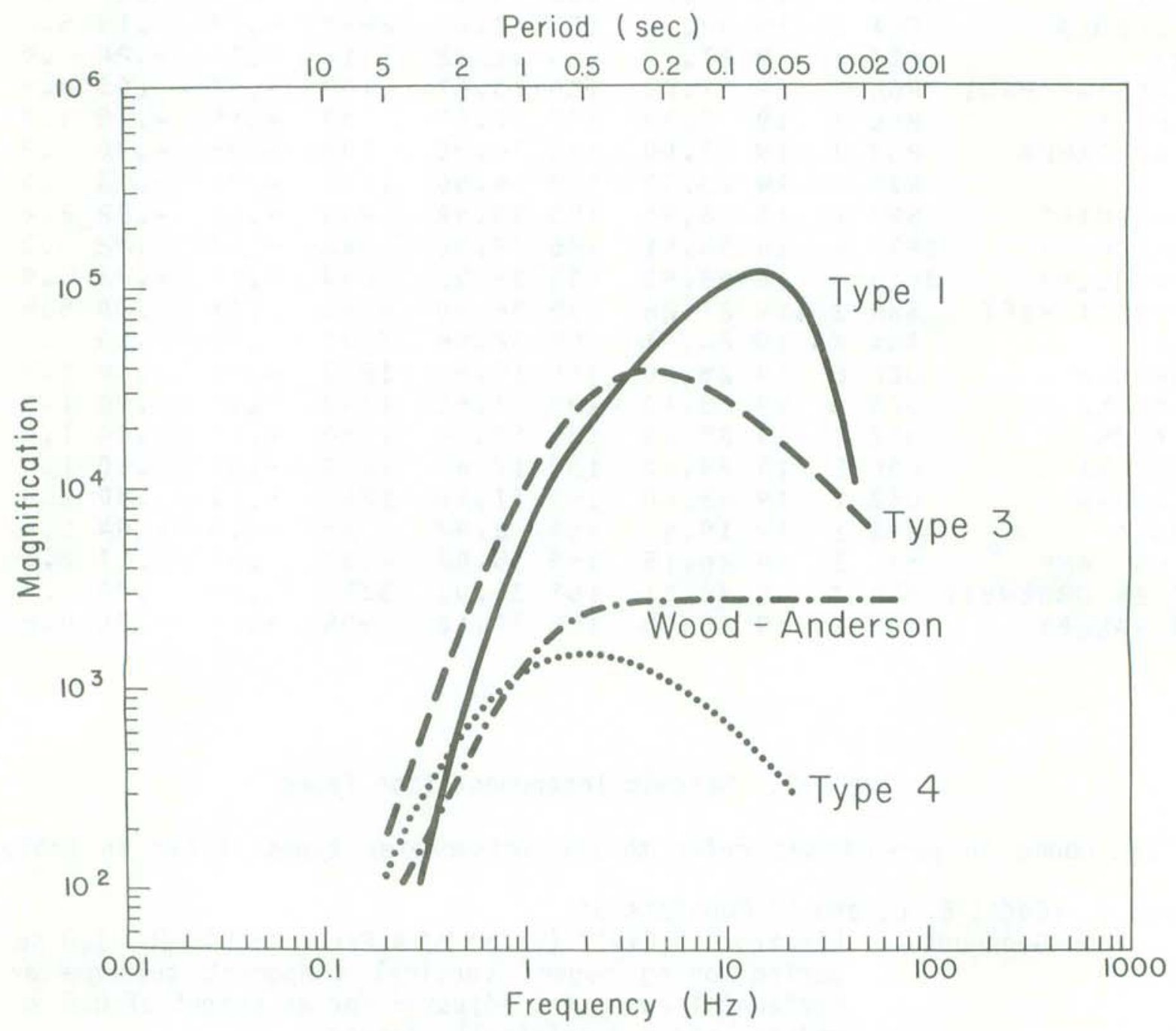

Figure 4. System response curves for the Wood-Anderson torsion seismograph and for the 3 different types of seismometers in use by the Hawaiian Volcano observatory. Types 3 and 4 are electro-mechanical seismographs recorded optically on photographic paper. Type 1 is the standard OEVE seismometer recorded on Develocorder film and magnetic tape. The curve for Type 1 includes response of the geophone, all electronics including telemetry, Develocorder galvanometer, and projection of $\mathrm{film}$ by a $20 \mathrm{x}$ viewer. The curves plot the unit response which should be multiplied by a constant but known factor (CAL), to get the reponse for an individual station. 


\section{SEISMIC DATA PROCESSING}

Develocorder films are scanned on a daily basis for earthquakes, and coda durations are measured for magnitude determination. Events are digitized, timed, and located on the Eclipse computer at HVO. Computer locations are made using the program HYPOINVERSE (Klein, F.W., Hypocenter location program HYPOINVERSE, U.S.G.S. Open file report 78-694, 1978), and problem events are reread and rerun. Magnetic tape copies of all arrival time and output summary data are kept in Menlo Park and HVO.

The crustal model used is specified by velocities at 4 depth points. Velocity at any depth is given by linear interpolation between points and uses a homogeneous half-space below.

\begin{tabular}{cr}
$\begin{array}{c}\text { VEL OCITY } \\
(\mathrm{km} / \mathrm{sec})\end{array}$ & \\
\hline 1.9 & DEPTH $(\mathrm{km})$ \\
6.5 & 0.0 \\
6.9 & 4.6 \\
8.3 & 15.0 \\
& 16.5
\end{tabular}

Two empirical sets of station delays or corrections were used in the locations, and are given in Table 1. Delay model 1 is used for events on Kilauea and its south flank, and delay model 2 applies to the rest of the island and offshore earthquakes. The delay models are in fact separated by a circle of radius $34 \mathrm{~km}$ centered at $19^{\circ} 22^{\prime} \mathrm{N}$ and $155^{\circ} 10^{\prime} \mathrm{W}$.

Magnitudes for most events were computed using both recorded amplitudes on low gain or Wood-Anderson stations, and signal or coda duration on selected short-period vertical stations. Amplitudes read from other than Wood-Anderson instruments are corrected to an equivalent Wood-Anderson amplitude using the curves of Figure 3 and CAL factors. Amplitude magnitudes larger than 2.5 are generally based on the Wood-Anderson instruments in Hilo or Type 4 seismographs at Uwekahuna. Smaller events may occasionally include amplitude readings from stations AHU, OTL, PPL, KHU, or WIL.

Duration magnitudes are determined from the length of signal in seconds read from the Develocorder viewer. This time, also called the "F-P time" is measured from the first $P$ arrival to the point where the earthquake signal has decayed nearly to the noise level. A bilinear relation is an appropriate fit to the data sample and is used to compute all duration magnitudes. Duration times are only read from Type 1 seismographs. Because duration magnitudes are relatively insensitive to station response and can be determined using the high-gain short-period stations, it is felt that duration magnitudes are more accurate and complete at the lower magnitudes (below 2).

The equations used in magnitude determination are:

$$
\begin{array}{ll}
\text { duration }<210 \mathrm{sec} & M=-5.2+3.89 \log (F-P)+.013 Z+.0037 \mathrm{D} \\
\text { duration }>210 \mathrm{sec} & M=-.905+2.026 \mathrm{log}(F-P)+.013 Z+.0037 \mathrm{D}
\end{array}
$$

where $Z$ and $D$ are the depth and epicentral distance in $k m$, respectively. 
The emphasis in both station coverage and detailed data analysis is on the highly active south half of the island of Hawaii. Hundreds of earthquakes too small to locate are counted daily, and the set of located earthquakes in the Kilauea region is nearly complete above magnitude about 2.0 . Many smaller events are located also. Substantial effort is made to locate earthquakes elsewhere on the island and within about $150 \mathrm{~km}$ of the island. Such coverage cannot be as complete as on the south flank, but nearly all events above magnitude 3.0 to 3.5 are located.

Data presented in the seismic summary is in four parts. Table 3 gives duration of harmonic tremor and numbers of earthquakes (most too small to locate) from several source regions around Kilauea. The source region is determined visually from signal character and pattern of arrival times at key stations. Maps showing computer located epicenters are given in Figures 8-13. The epicenter maps are on different scales, and show both all located earthquakes and large events only.

The list of computer locations constitutes the bulk of this summary, and is given in Table 5. Each earthquake in the list is assigned a three-letter code based on its location and depth. Figures 4-7 are maps of the regions used to assign the location codes. The latitude and longitude limits of rectangular regions are listed in Table 4. When the listed coordinates imply an overlap, precedence is given according to Figures 4-7. Table 6 relists the events in Table 5 for which either duration or amplitude magnitude is 3.0 or larger. It is felt that this list is a more objective measure of large earthquakes than a list of felt earthquakes.

Table 3. Number of earthquakes and minutes of tremor recorded on seismographs around Kilauea.

Earthquake categories are:

1) Kilauea summit, short period caldera: shallow earthquakes beneath the caldera.

2) Kilauea summit, long period caldera: earthquakes characterized by low frequency signatures, often originating $5-10 \mathrm{~km}$ below the caldera.

3) Kilauea summit $30 \mathrm{~km}$ : deep earthquakes about $30 \mathrm{~km}$ beneath the summit region.

4) Kaoiki and southwest rift: earthquakes beneath the southwest rift of Kilauea, western parts of the Koae faults and adjacent Kaoiki fault system.

5) Upper east rift: earthquakes in the upper and middle east rift zone, the adjacent parts of the south flank, and eastern parts of the Koae faults.

6) Lower east rift: earthquakes in the lower east rift zone and adjacent parts of the south flank.

7) Offshore PPL: earthquakes from offshore areas south of the Puu Pili station, including Loihi seamount.

8) Mauna Loa long period: low frequency events near Mauna Loa summit.

9) Mauna Loa short period: shallow earthquakes in the Mauna Loa caldera region.

Tremor is separated into four categories: shallow, intermediate and deep Kilauea, and Mauna Loa. Depth is inferred on the basis of relative ampititudes on seismographs. 
I ISHORT LONG I KAO, UP. LOW. OFF = I

KILAUEA MAUNAI

I 1983 I CALDERA KM I RIFT RIFT RIFT PPL IPER. PFR. T SHAL INT DEFP LOA I

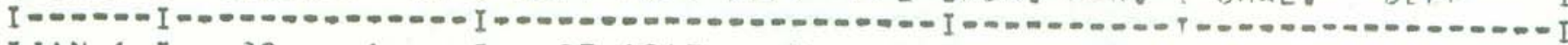

I

I. 4 I 10

$\begin{array}{lllll}29 & 1 & I & 23 & 1963\end{array}$

I 5 I 326

1

I

$30 \quad 5977$

3 I 401348

$40 \quad 2709$

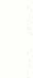

$$
\text { I }
$$

I

$$
\text { I }
$$

$$
\text { I }
$$

$$
\text { I }
$$$$
\text { I }
$$$$
\text { I }
$$$$
\text { I }
$$$$
\text { I }
$$

$$
\begin{aligned}
& \mathrm{I} \\
& \mathrm{I} \\
& \mathrm{I} \\
& \mathrm{I} \\
& \mathrm{I} \\
& \mathrm{I} \\
& \mathrm{I} \\
& \mathrm{I}
\end{aligned}
$$

$$
\text { trats }
$$

I

$$
\text { I }
$$$$
\text { (1) }
$$

$$
\text { I }
$$




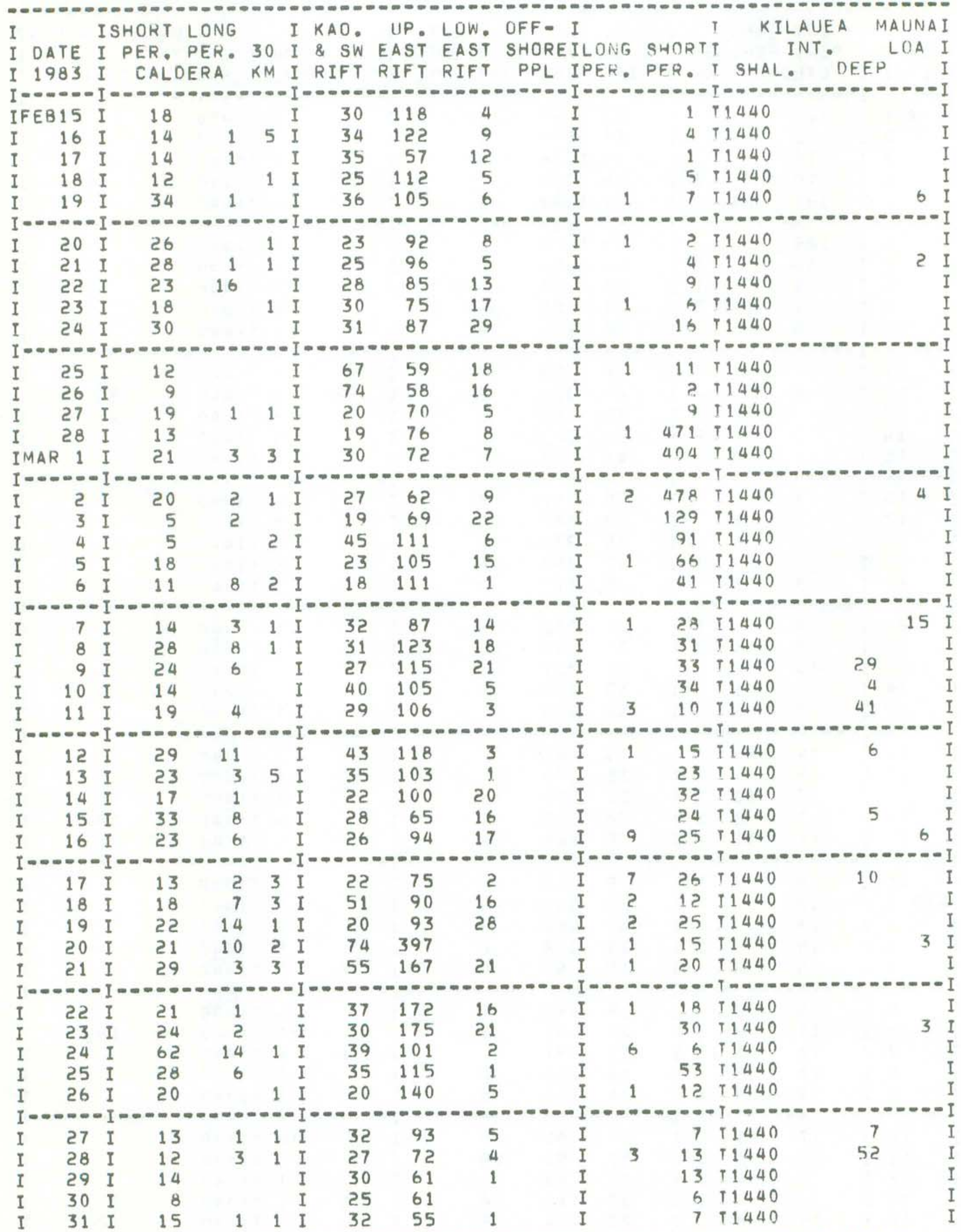


I ISHORT LONG I KAO. UP. LOW. OFF- I

KILAUEA MAUNAI

I DATE I PER. PER. 30 I \& SW EAST EAST SHOREILONG SHORTT INT \& LOA I

I 1983 I CALDERA KM I RIFT RIFT RIFT PPL IPER. PFR. I SHAL. DEEP I

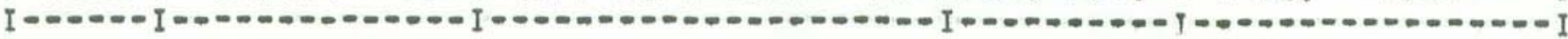

$\begin{array}{lllllllll}I A P R & 1 & I & 12 & 2 & 1 & I & 27 & 51\end{array}$

I

5 I 32

5 I

$34 \quad 45$

31

14

6 I

1952

$\begin{array}{llllll}37 & 13 & 1 & I & 23 & 42\end{array}$

4

$12+1440$

4 I

5313

$\begin{array}{llll}57 & 40 & 11 & 11\end{array}$

$\begin{array}{llll}57 & 40 & 11 & 11\end{array}$

$10 T 1440$

$1=0 .-1-2$

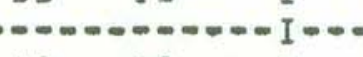

1311440$$
\text { I }
$$

6 I

$42 \quad 10$

$26 \quad 51$

7 I

48

311

34

28

15

69

2

10

9 I

10

$1041 \mathrm{I}$

$34 \quad 114$

10

10

$\begin{array}{llll}I & & 10 & 11440 \\ I & 1 & 13 & 11440 \\ I & 6 & 25 & 11440 \\ I & & 33 & I 1440\end{array}$

10 I 1232 2 I

$20 \quad 110$

I

$33 T 1440$

$\begin{array}{llll}17 & 3 & 15 & I\end{array}$

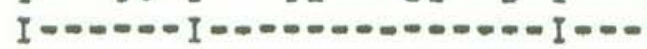

I

$\begin{array}{lllll}12 & I & 23 & 10\end{array}$

131

17

141

13

8

$27 \quad 198$

16

$\begin{array}{lll}39 & 285 & 12\end{array}$

20 296

39222

12
4

5

$17 \quad 250 \quad 10$

$1=0.0-01-017$
1

I

I

I

I

I

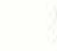

I

I

I

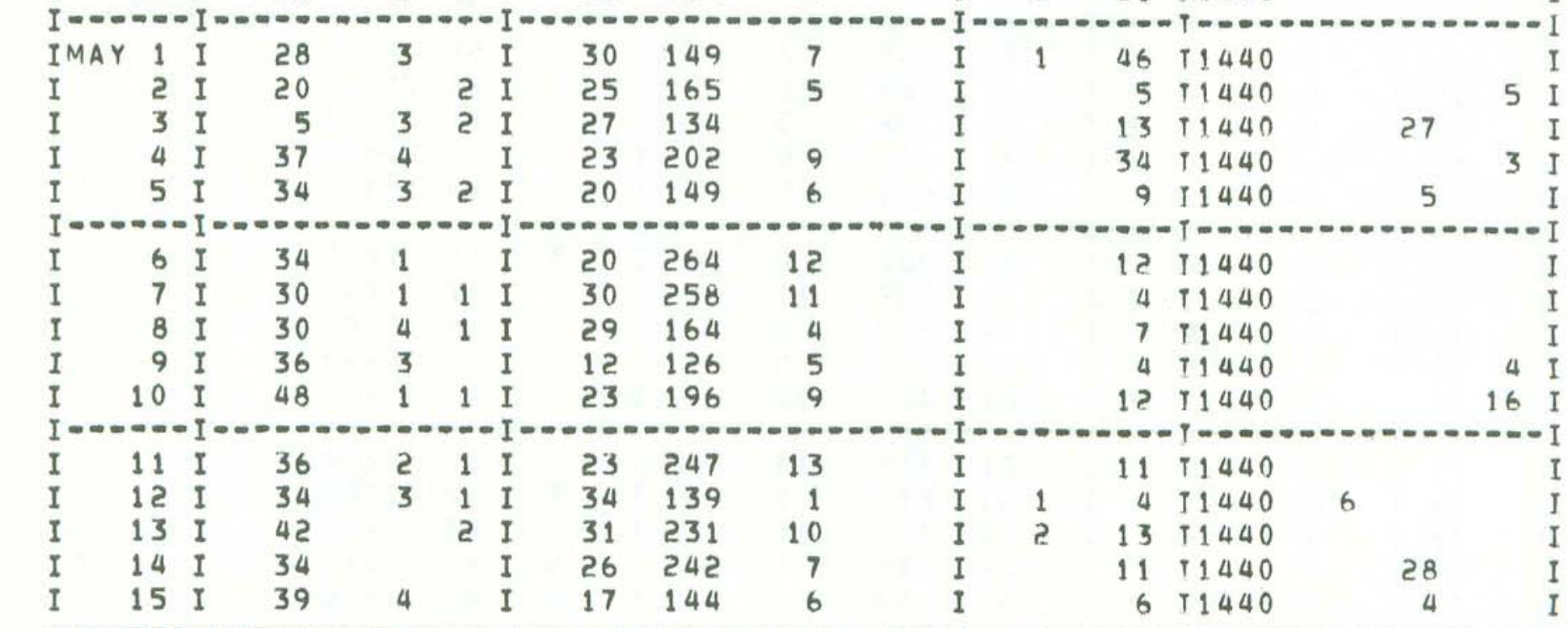


I DATE I PER. PER. 30 I \& SW EAST EAST SHOREILONG SHORTT IOAT. I

I 1983 I CALDERA KM I RIFT RIFT RIFT PPL IPER. PER I SHAL. DEEP I

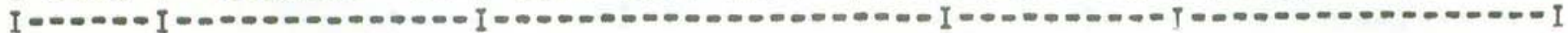

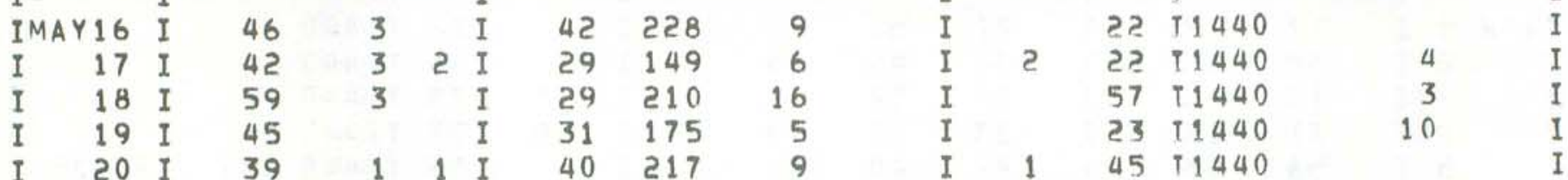

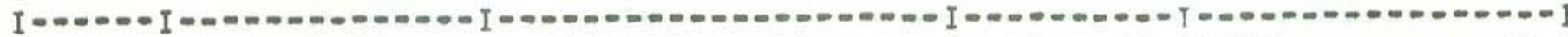

$\begin{array}{llllllllllll}I & 21 & I & 35 & I & 23 & 234 & 11 & I & 3 & 40 & I 1440\end{array}$

$\begin{array}{llllllllll}22 & I & 38 & 1 & 1 & I & 22 & 129 & 5 & I\end{array}$

$\begin{array}{lllllllllll}23 & 1 & 65 & I & 21 & 189 & 10 & I & 1 & 23 & \text { J } 1440\end{array}$

$\begin{array}{llllllllll}24 & 1 & 71 & 5 & I & 17 & 222 & 13 & I & 35\end{array}$

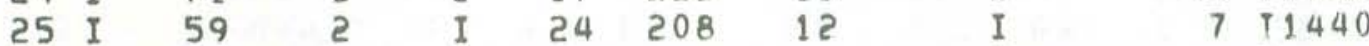

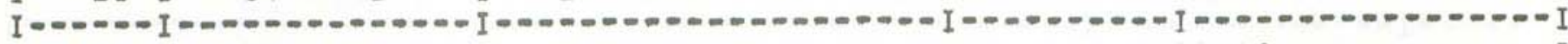

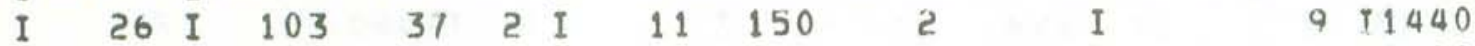

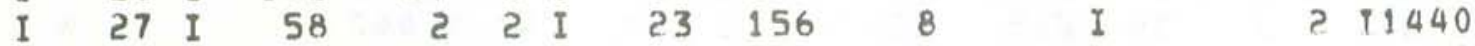

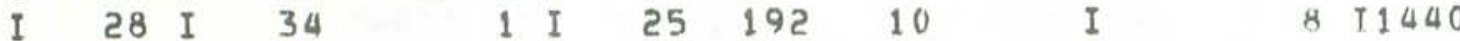

$\begin{array}{rrrrrrrrrrrrrrrr}I & 29 & I & 26 & 1 & 2 & I & 24 & 181 & 8 & I & 11440 & 4 & 1 \\ I & 30 & I & 39 & & 1 & I & 27 & 243 & 14 & I & 3 & 10 & T 1440 & \end{array}$

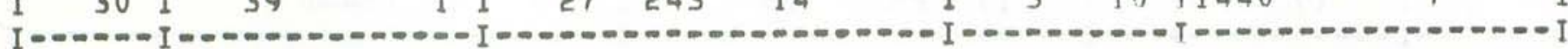

$\begin{array}{llllllllllllll}I & 31 & I & 37 & 1 & I & 27 & 203 & 2 & I & 1 & 14 & T 440 & I\end{array}$

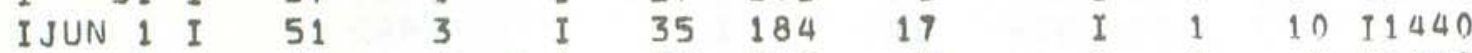

$\begin{array}{rrrrrrrrrrrrrrrr}I & 2 & I & 45 & 1 & 3 & I & 33 & 127 & 4 & I & 1 & 71440 & I \\ I & 3 & I & 73 & 2 & & I & 32 & 176 & 6 & I & 1 & 36 & 11440 & 9\end{array}$

$\begin{array}{llllllllllllll}I & 4 & I & 119 & 5 & I & 25 & 197 & 14 & I & 10 & 54 & 1440 & \end{array}$

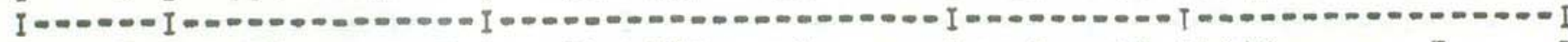

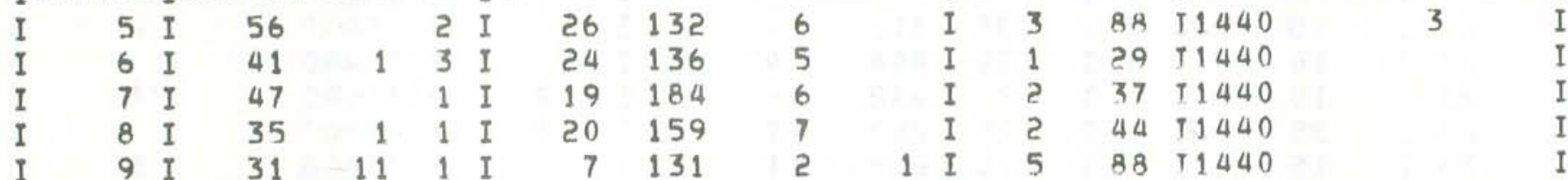

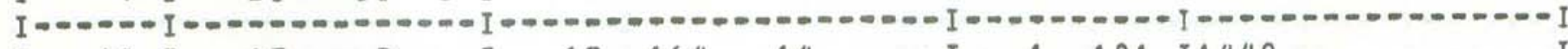

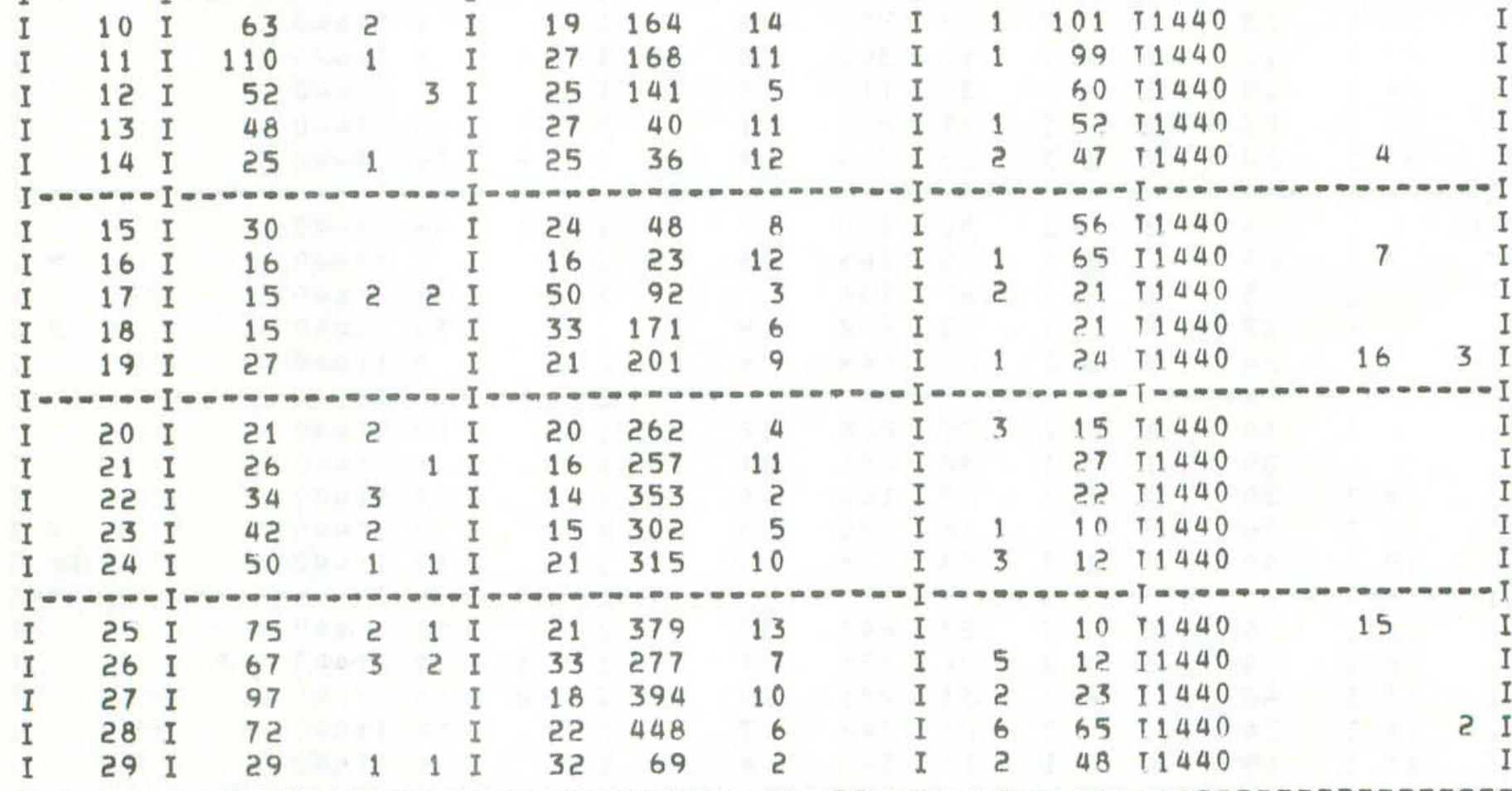




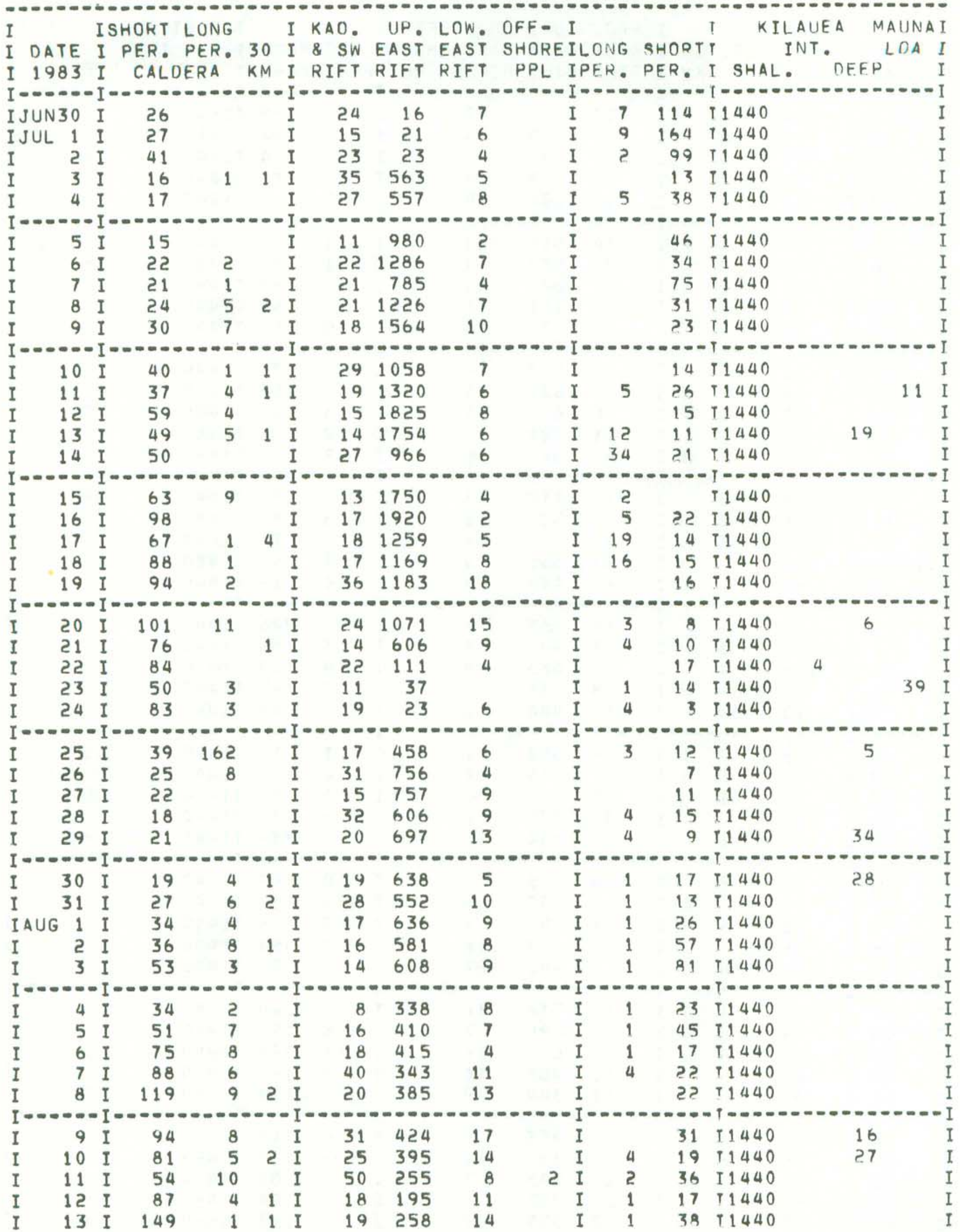




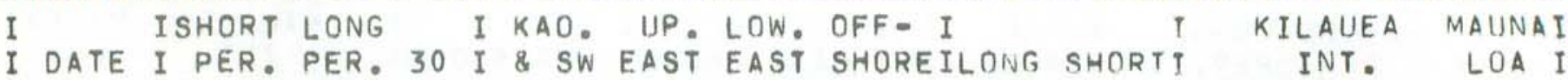

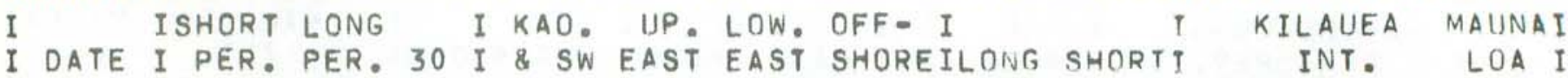

I 1983 I CALDERA KM I RIFT RIFT RIFT PPL IPER. PER. T SHAL. DEEP I I - - - - - I I - - D.

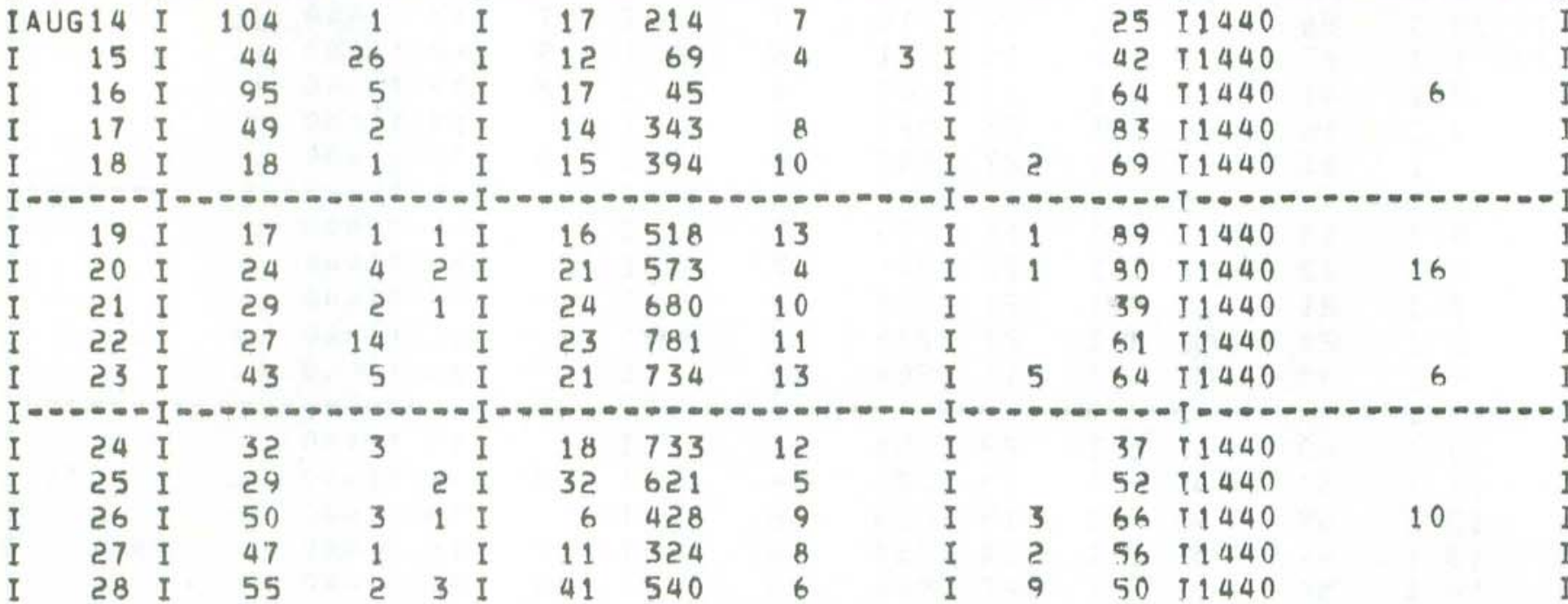

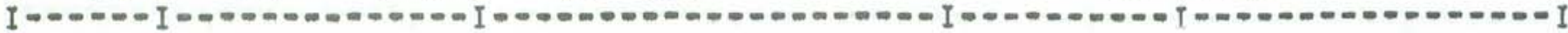

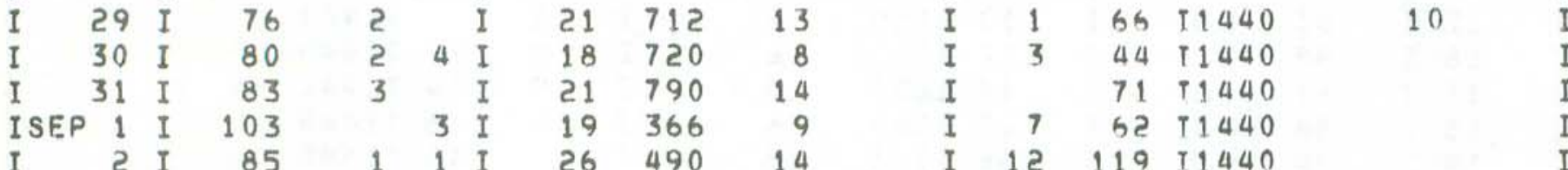

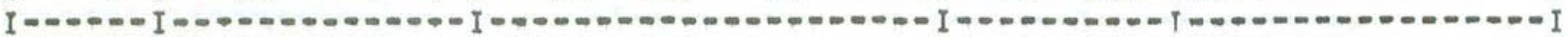

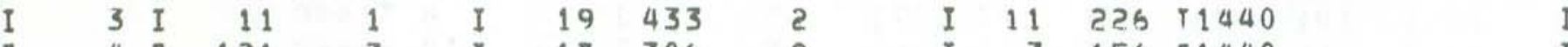

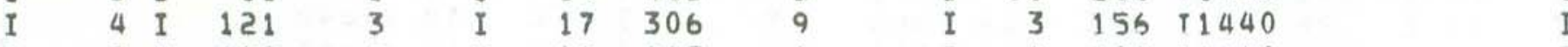

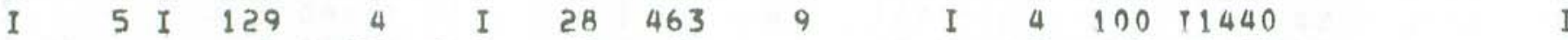

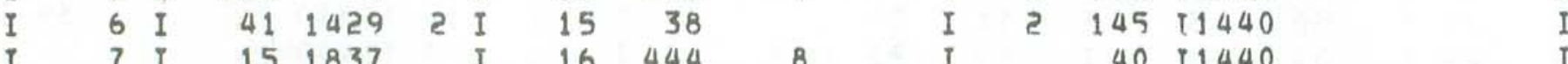

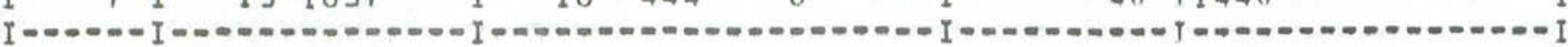

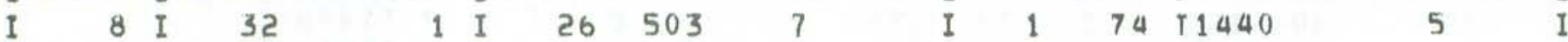

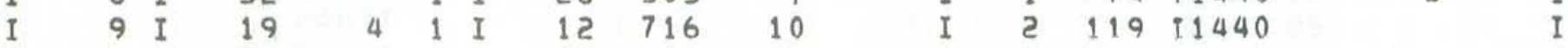

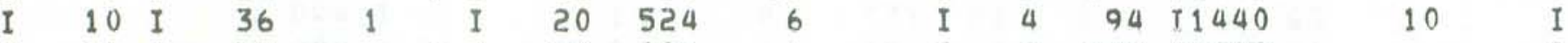

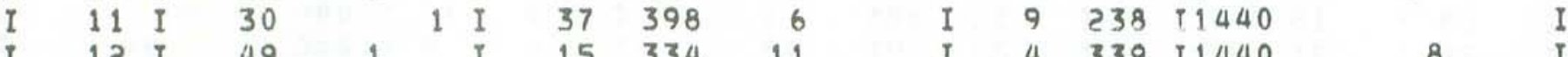

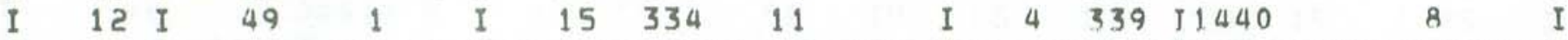

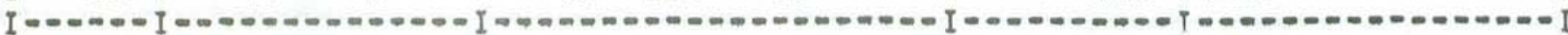

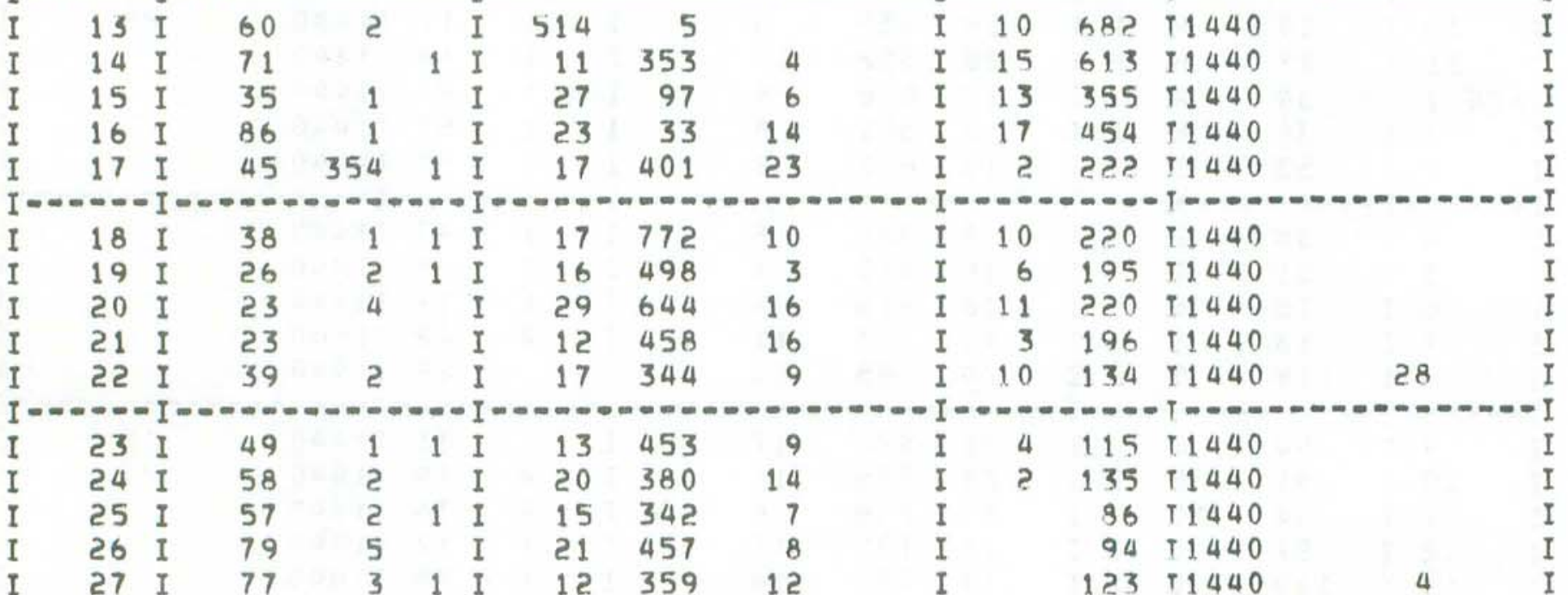




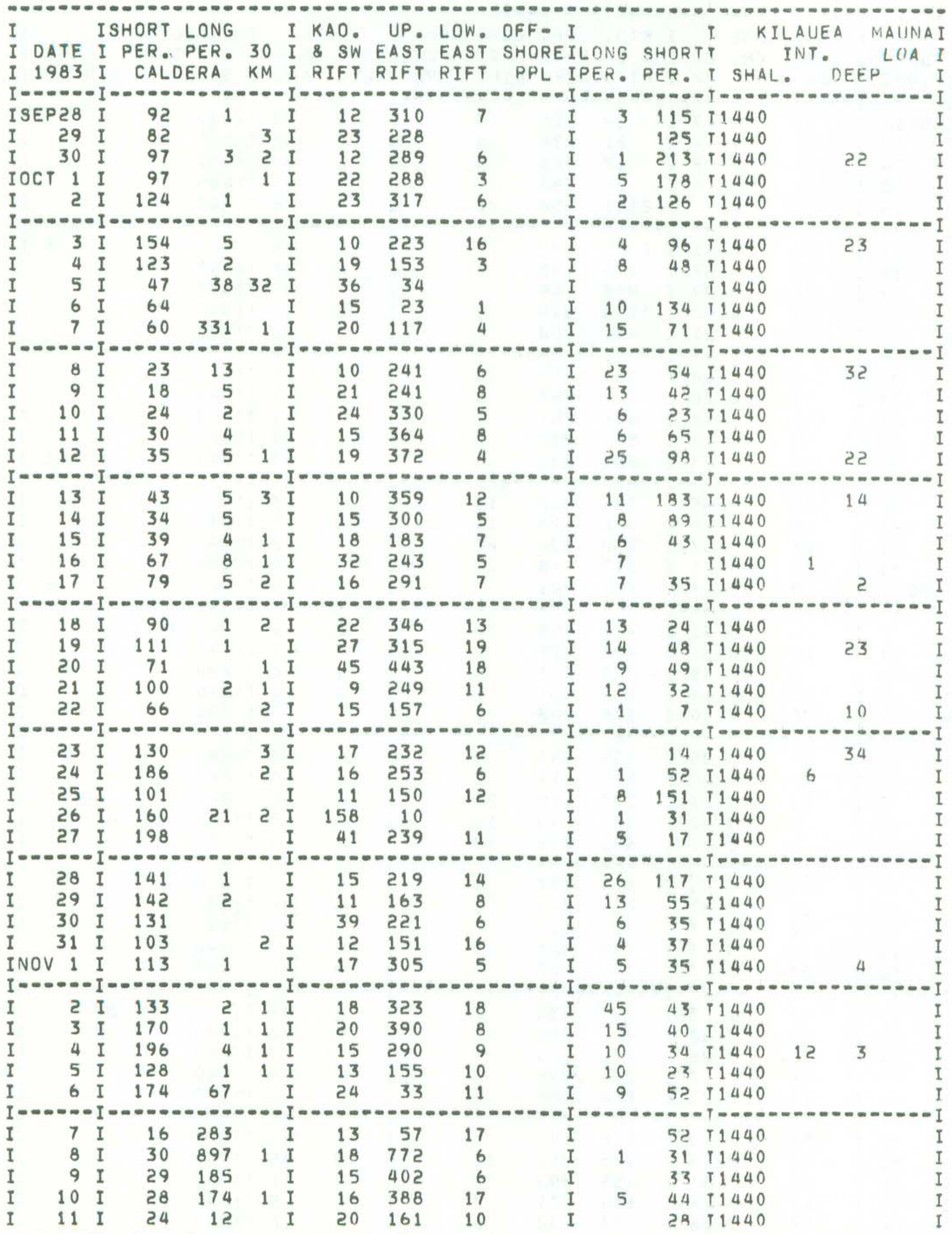




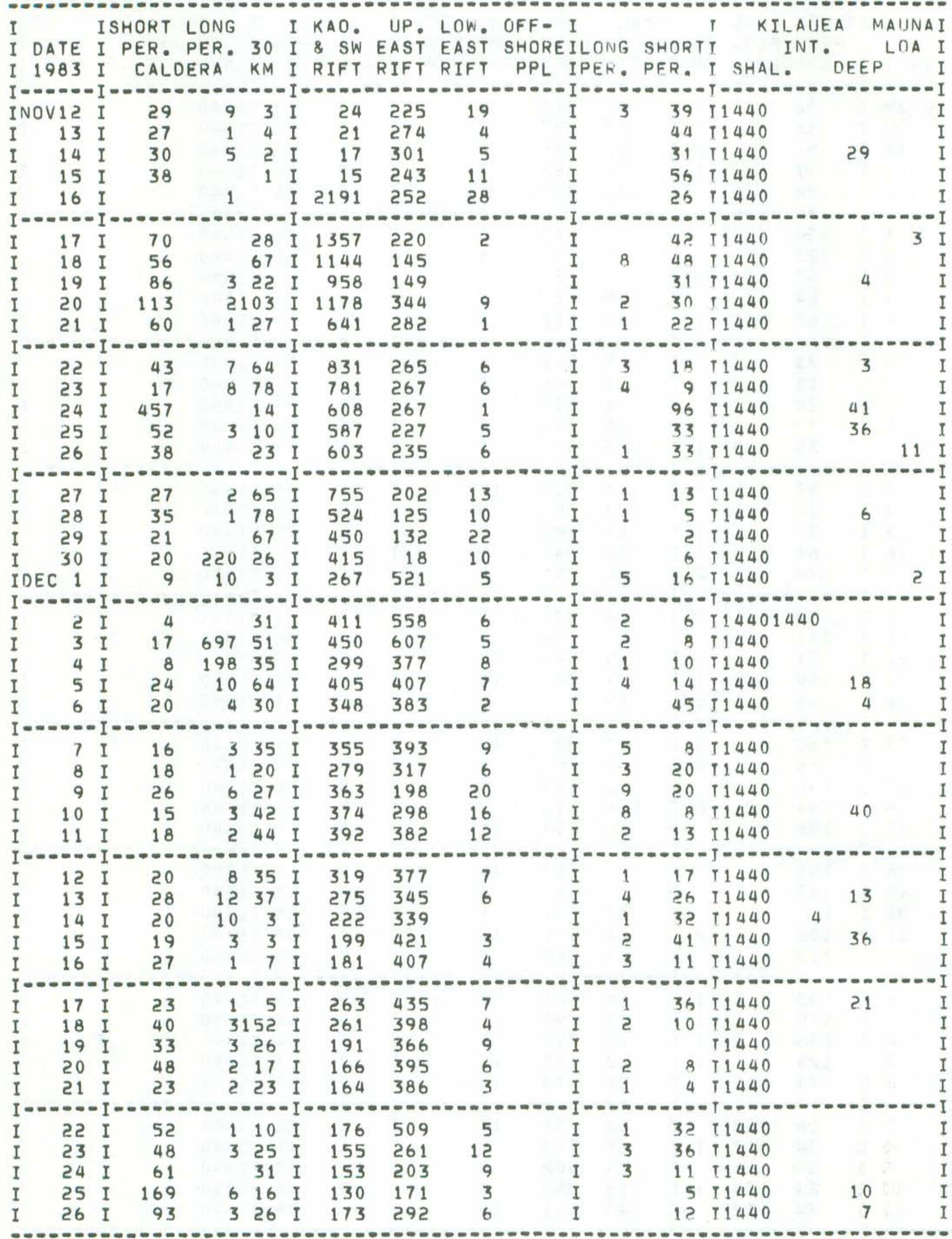




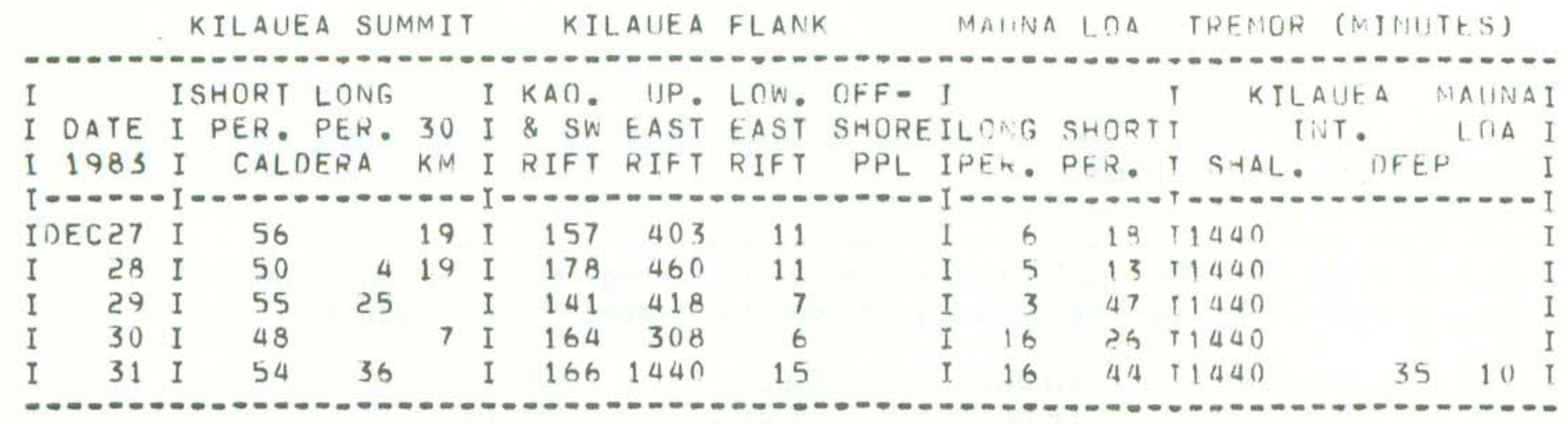

Table 4. Coordinates of named earthquake regions.

--All earthquakes are in one of the following groups.

--Identified by a numerical class or 3-letter code:

--Shal low:

1 SNC - Shallow north caldera $(0-5 \mathrm{~km})$

2 SSC - Shallow south caldera $(0-5 \mathrm{~km})$

3 SEC - Shallow east caldera $(0-5 \mathrm{~km})$

4 SER - Shallow east rift $(0-5 \mathrm{~km})$

5 SME - Shallow middle east rift $(0-5 \mathrm{~km})$

6 KOA - Koae fault zone $(0-5 \mathrm{~km})$

7 SSF - Shallow south flank (0-5 km)

8 SLF - Shallow lower east rift (0-5 km)

--Intermediate depth:

9 SF1 - Kilauea south flank (5-13 km) (west end)

10 SF2 - Kilauea south flank (5-13 km)

11 SF3 - Kilauea south flank $(5-13 \mathrm{~km})$

12 SF4 - Kilauea south flank $(5-13 \mathrm{~km})$

13 SF5 - Kilauea south flank (5-13 km) (east end)

14 LER - Lower east rift (5-99 km)

15 ML 0 - Mauna Loa $(0-13 \mathrm{~km})$

16 LSW - Lower SW rifts of Kilauea \& Mauna Loa (0-13 km)

17 GLN - Glenwood $(0-13 \mathrm{~km})$

18 SWR - SW rift $(0-13 \mathrm{~km})$

19 INT - Intermediate caldera (5-13 km)

20 KAO - Kaoiki $(0-13 \mathrm{~km})$

--Deep:

21 DEP - Deep Kilauea (>13 km) (below regions 1-13, 17-19)

22 DLS - Deep lower SW rift $(>13 \mathrm{~km})$ (below region 14)

23 DML - Deep Mauna Loa (>13 km) (below regions 15, 20)

--0uter regions, all depths:

24 LOI - Loihi (a11 depths)

25 KON - South Kona (a11 depths)

26 HUA - Hualalai (all depths)

$27 \mathrm{KOH}$ - Kohala (all depths)

28 KEA - Mauna Kea (a11 depths)

29 HIL - Hilo (all depths)

30 DIS - Distant, everywhere else 
Table 4 (continued)

--The latitude and longitude limits of the regions are given below.

- When the coordinates imply an overlap, precedence is given as in the maps.

\begin{tabular}{|c|c|c|c|c|c|}
\hline No. & Code & N.l at. & S.L at. & W.L on. & E.Lon. \\
\hline 1 & SNC & 1928 & 1924.5 & 15519 & $155 \quad 14$ \\
\hline 2 & SSC & 1924.5 & 1922 & 15519 & 15516.5 \\
\hline 3 & SEC & 1924.5 & 1922 & 15516.5 & 15514 \\
\hline 4 & SER & 1926 & 1920.5 & 15514 & 15507.2 \\
\hline 5 & SME & 1926 & ----- & $\begin{array}{lll}155 & 07.2\end{array}$ & 15500 \\
\hline 6 & $\mathrm{KOA}$ & 1922 & 19. 20.5 & 15517 & $155 \quad 14$ \\
\hline 7 & SSF & $-\cdots$ & 1910 & 15517 & 15500 \\
\hline 8 & SLE & 1932 & 1916 & 15500 & 15440 \\
\hline 9 & SF1 & 1922 & 1910 & 15517 & $155 \quad 14.5$ \\
\hline 10 & SF2 & 1926 & 1910 & $155 \quad 14.5$ & $155 \quad 12.3$ \\
\hline 11 & SF3 & 1926 & 1910 & 15512.3 & 15509.1 \\
\hline 12 & SF4 & 1926 & 1910 & $\begin{array}{ll}155 & 09.1\end{array}$ & 15505.3 \\
\hline 13 & SF5 & 1926 & 1910 & 15505.3 & 15500 \\
\hline 14 & LER & 1932 & 1916 & 15500 & 15440 \\
\hline 15 & ML 0 & 1943 & 1919 & 15535 & $155 \quad 19$ \\
\hline 16 & L SW & 1919 & 1840 & 15543 & 15525 \\
\hline 17 & GL N & 1943 & 1926 & $155 \quad 19$ & 15500 \\
\hline 18 & SWR & 1922 & 1910 & 15525 & $\begin{array}{ll}155 & 17\end{array}$ \\
\hline 19 & INT & 1928 & 1922 & 15519 & 15514 \\
\hline 20 & KAO & 1930 & 1919 & 15532 & 15519 \\
\hline 21 & DEP & 1943 & 1910 & 15525 & 15500 \\
\hline 22 & DLS & 1919 & 1840 & 15543 & 15525 \\
\hline 23 & DML & 1943 & 1919 & 15535 & 15519 \\
\hline 24 & LOI & 1910 & 1840 & 15525 & 15500 \\
\hline 25 & KON & 1939 & 1900 & 15620 & 15543 \\
\hline 26 & HUA & 1955 & 1939 & 15620 & 15543 \\
\hline 27 & $\mathrm{KOH}$ & 2025 & 1955 & 15620 & 15534 \\
\hline 28 & KEA & 2025 & 1943 & 15543 & $154 \quad 40$ \\
\hline 29 & HIL & 1947 & 1932 & 15509 & 15440 \\
\hline
\end{tabular}


Figure 5. Earthquake classification, shallow 0-5 km deep Kilauea and east flank Mauna Loa.

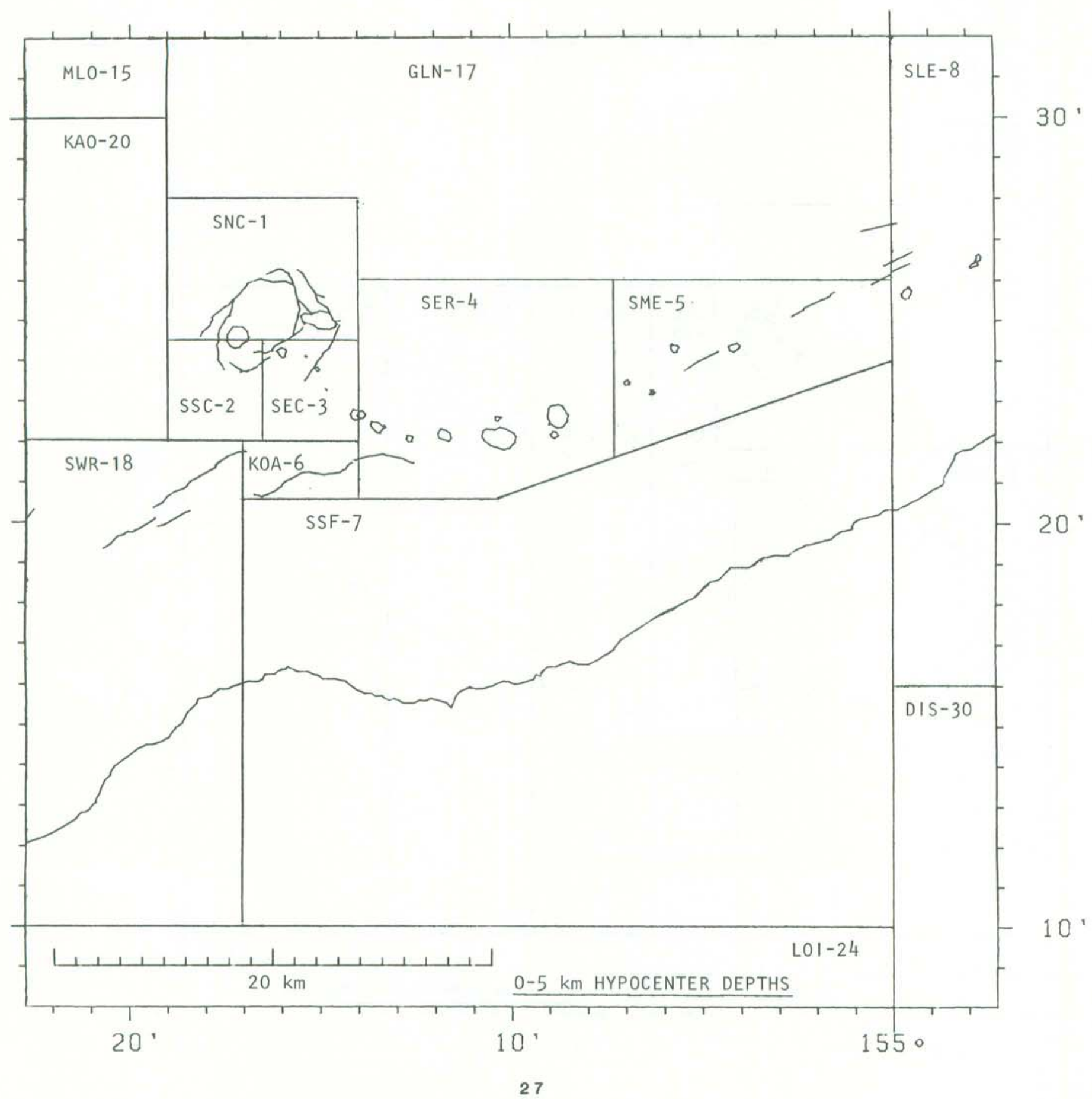


Figure 6. Earthquake classification, intermediate 5-13 km Kilauea and east flank Mauna Loa.

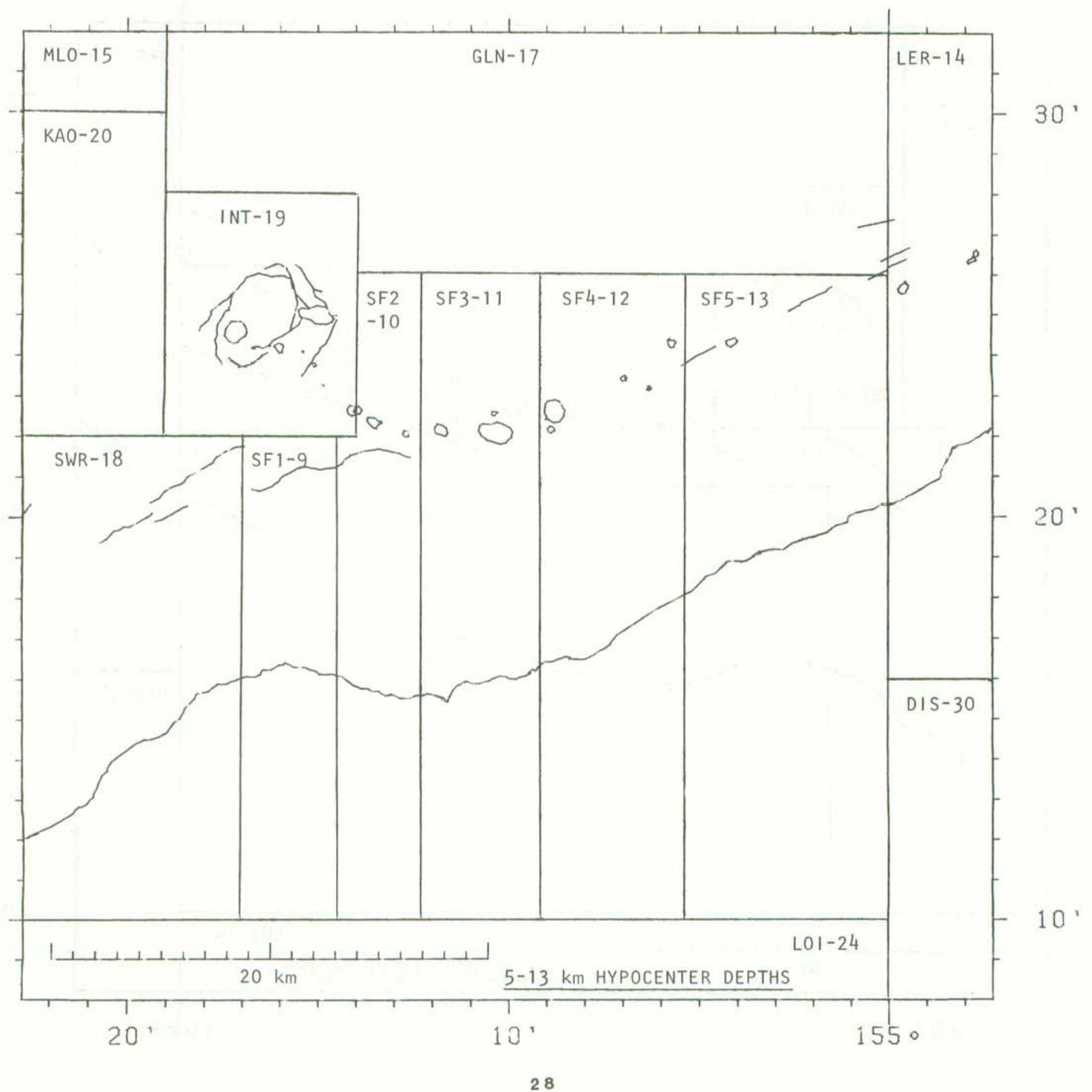


Figure 7. Earthquake classification, crustal 0-13 km deep island of Hawaii.

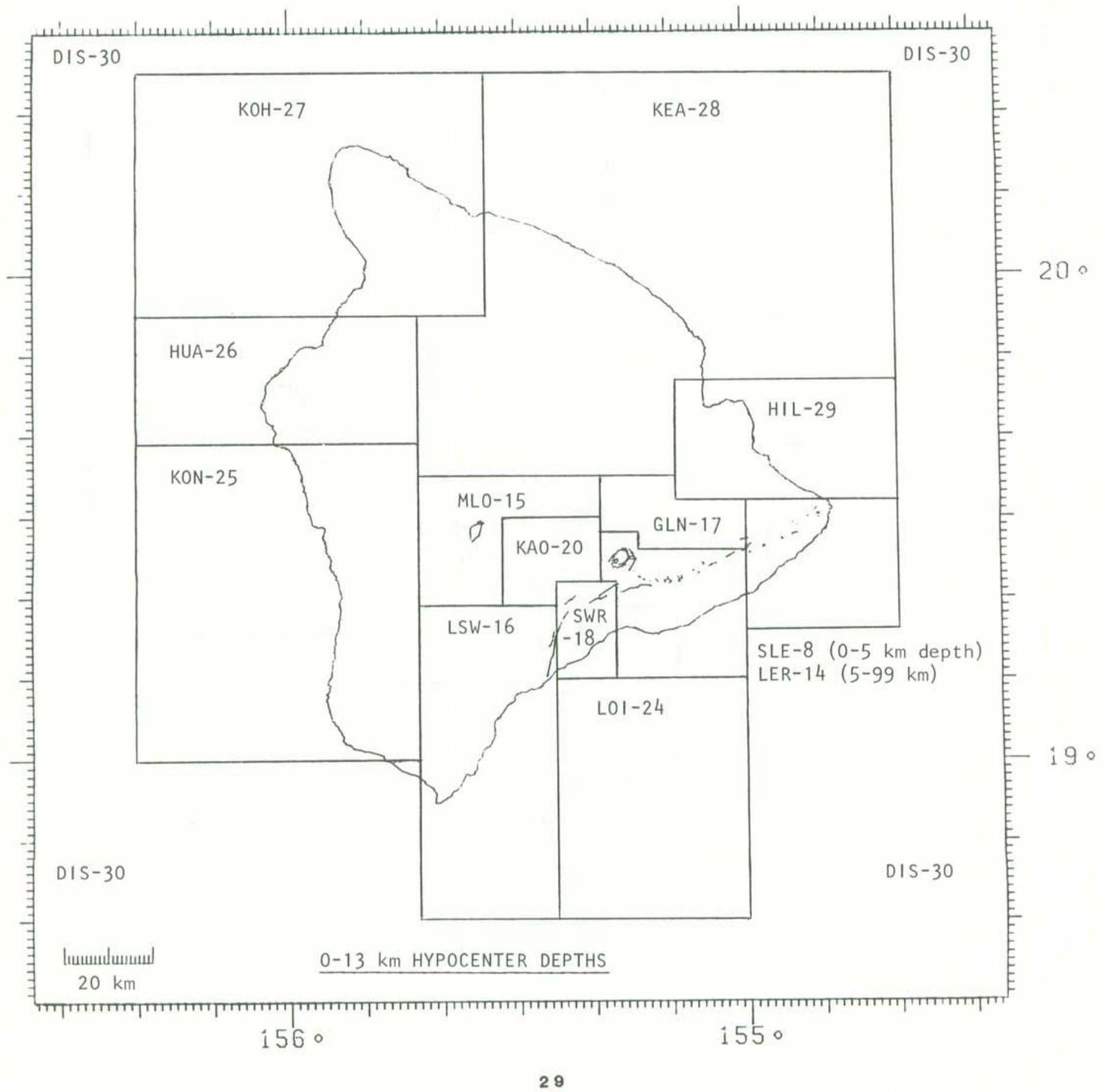


Figure 8. Earthquake classification, mantle greater than $13 \mathrm{~km}$ deep island of Hawaii.

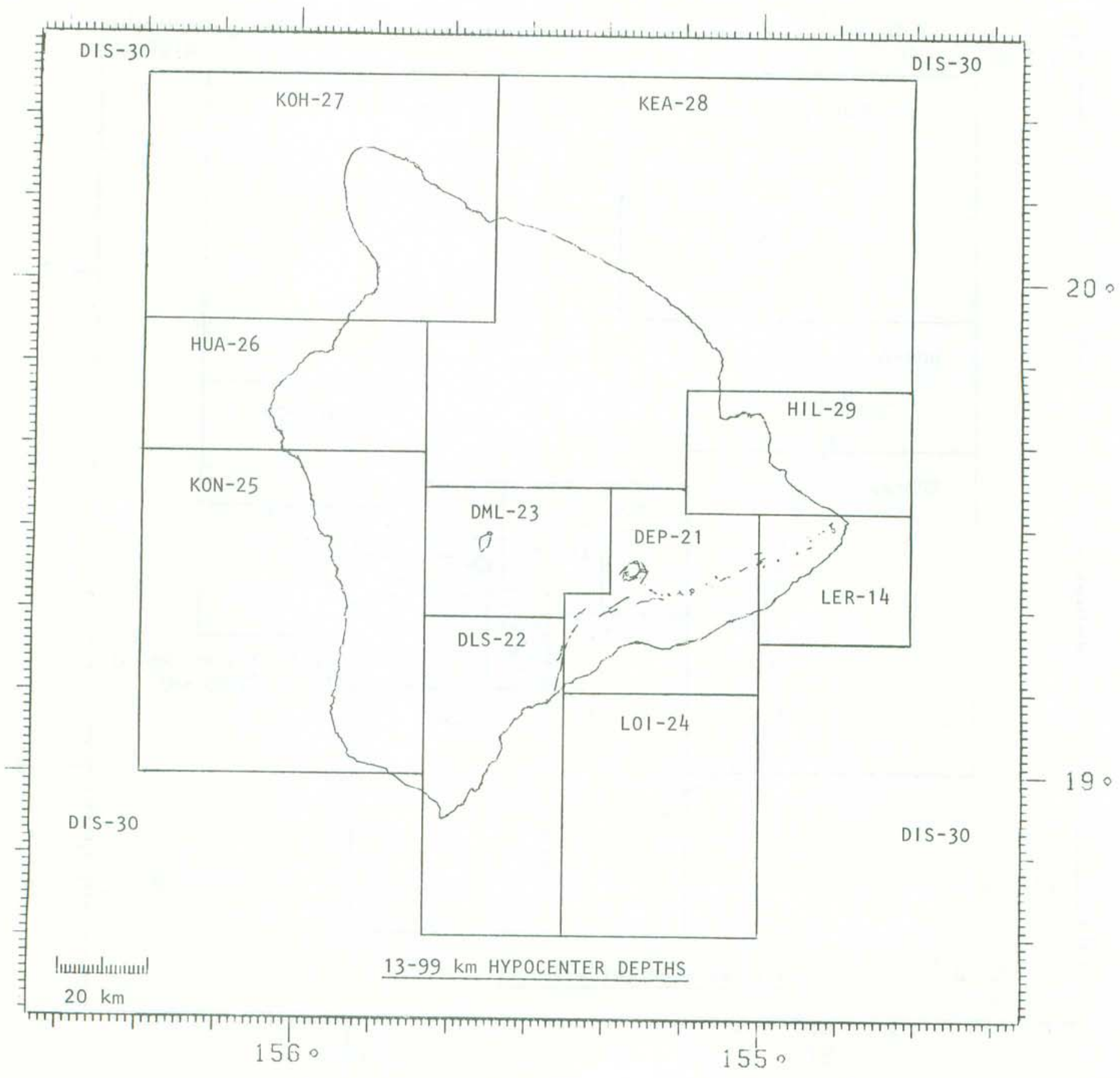


Figure 9

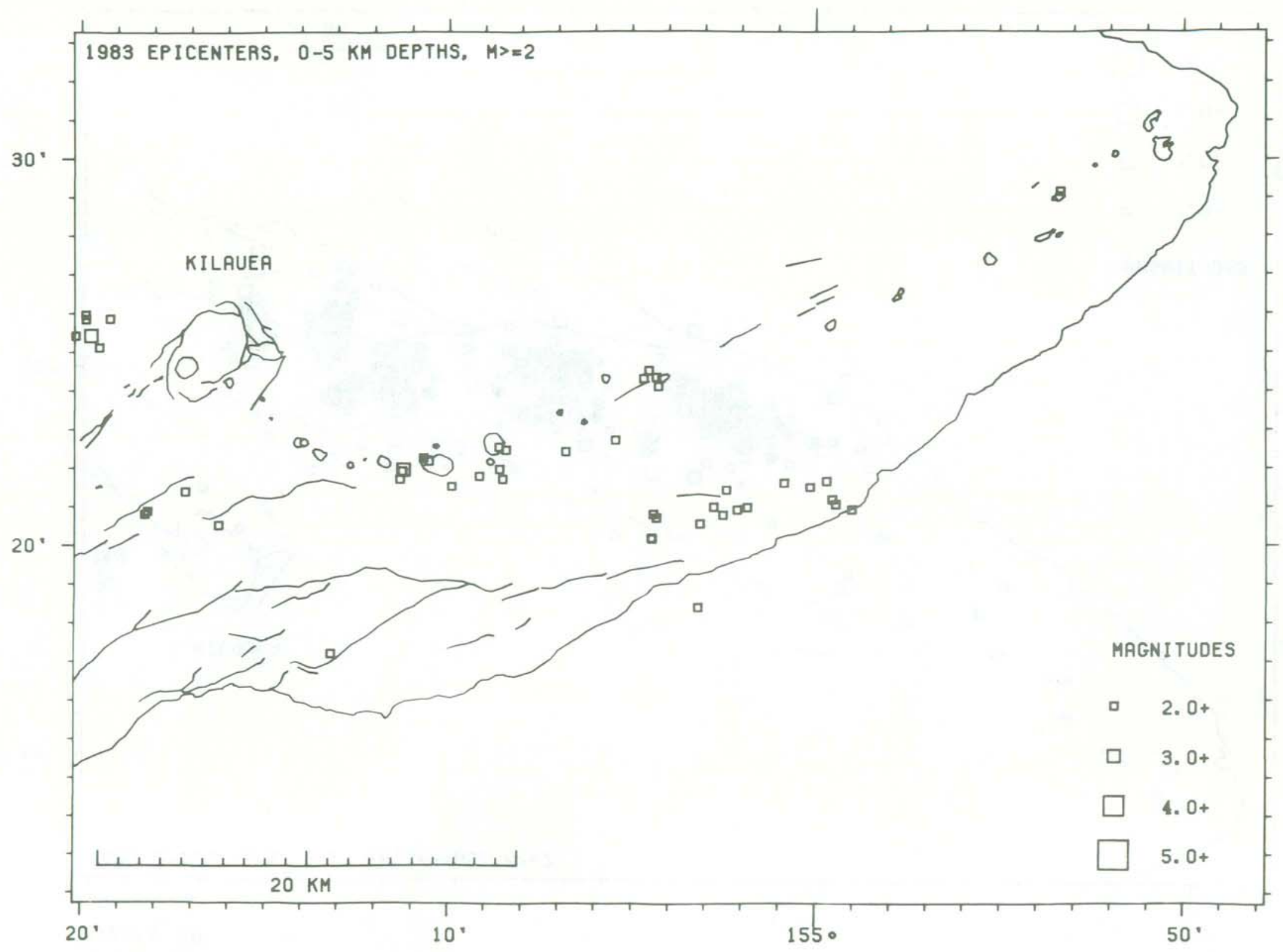


Figure 10

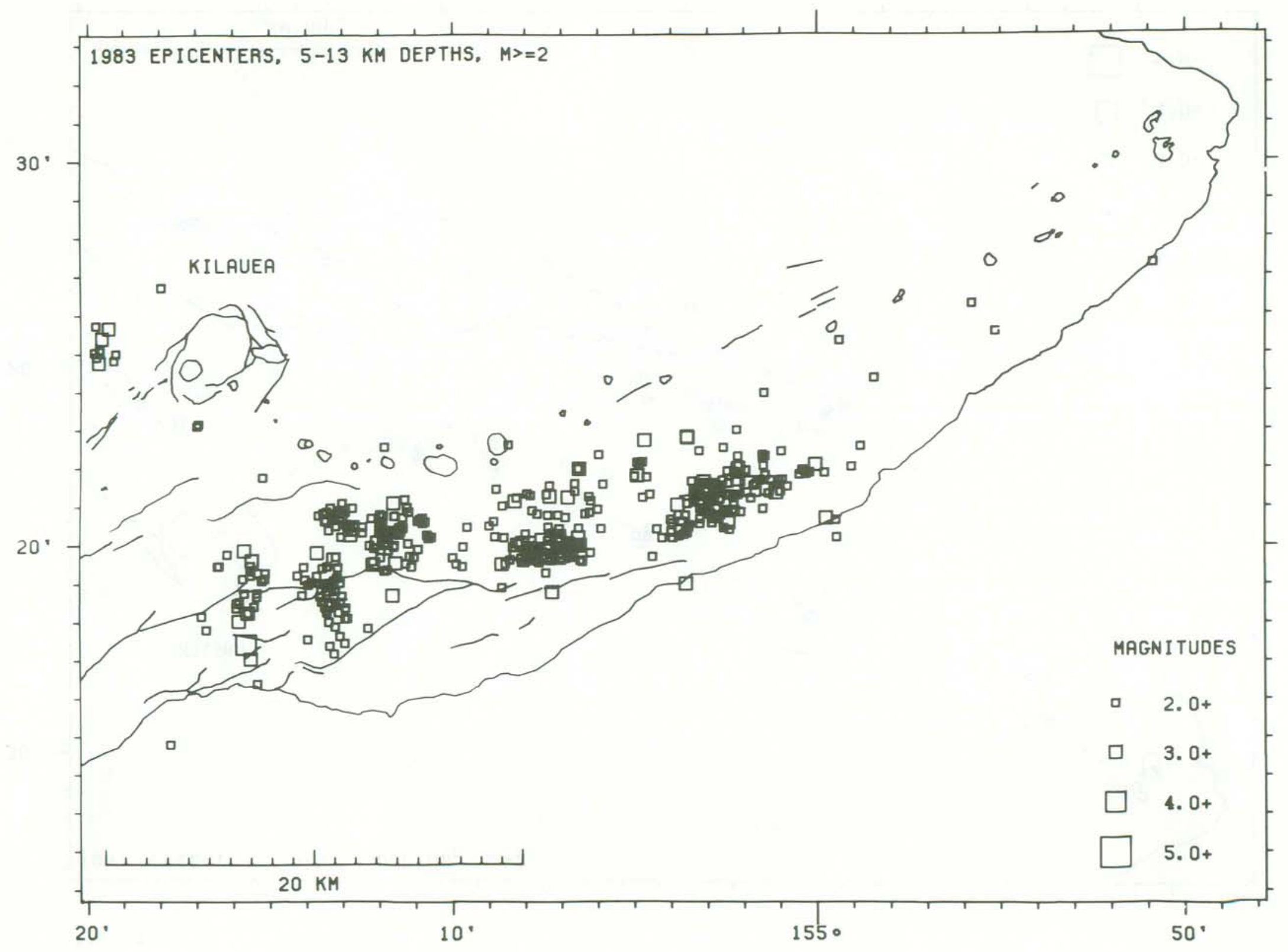


Figure 11

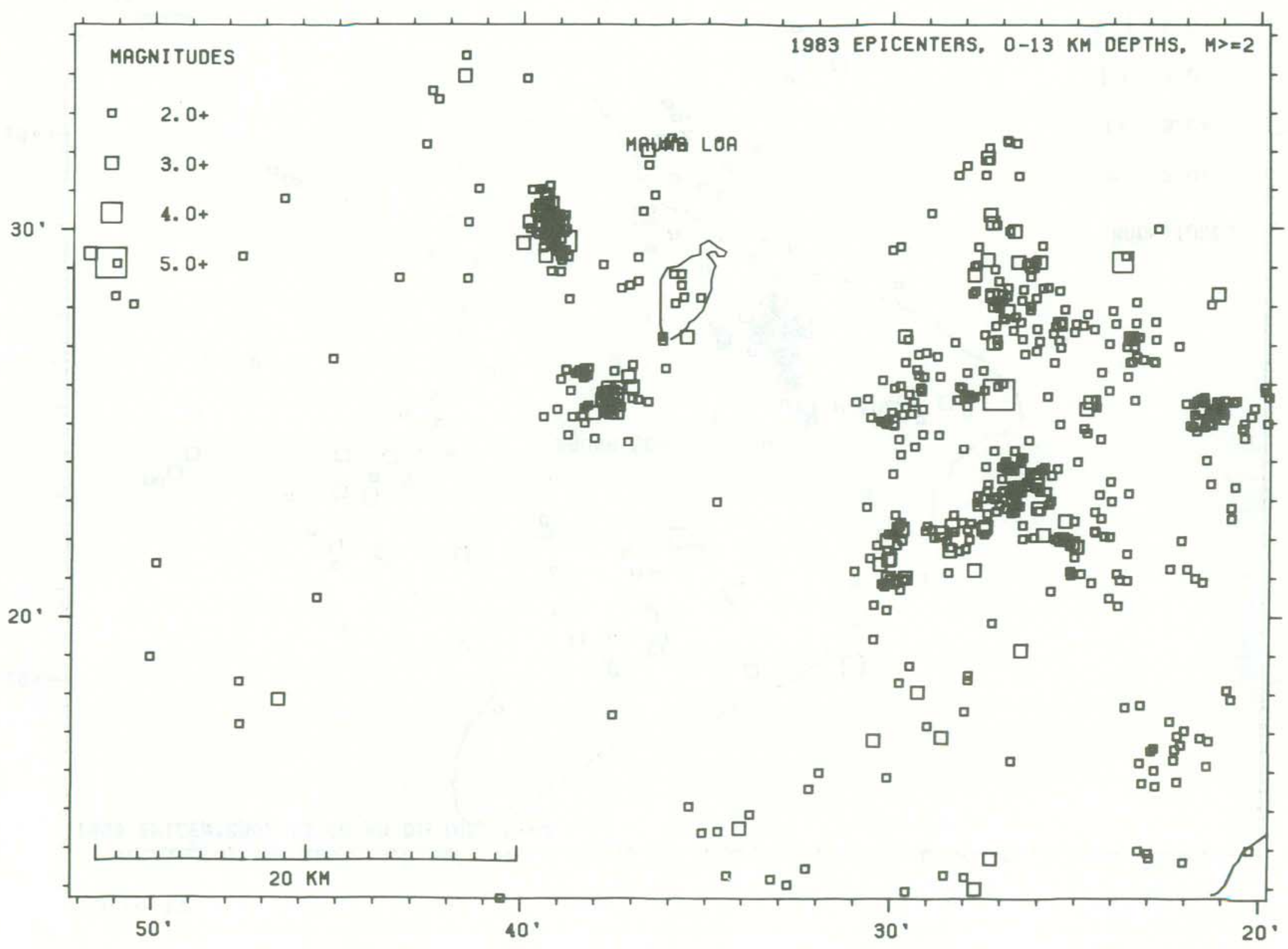


Figure 12

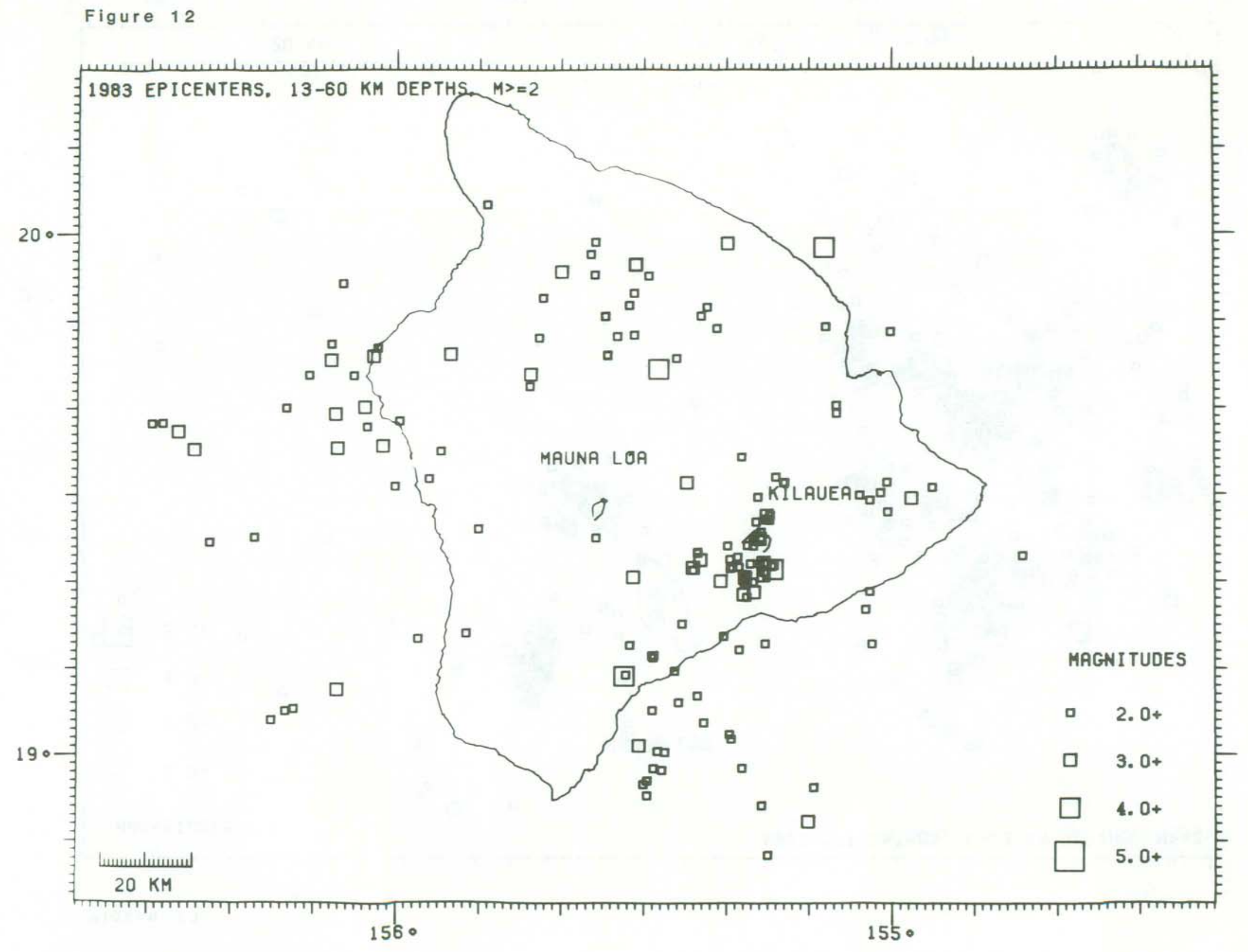




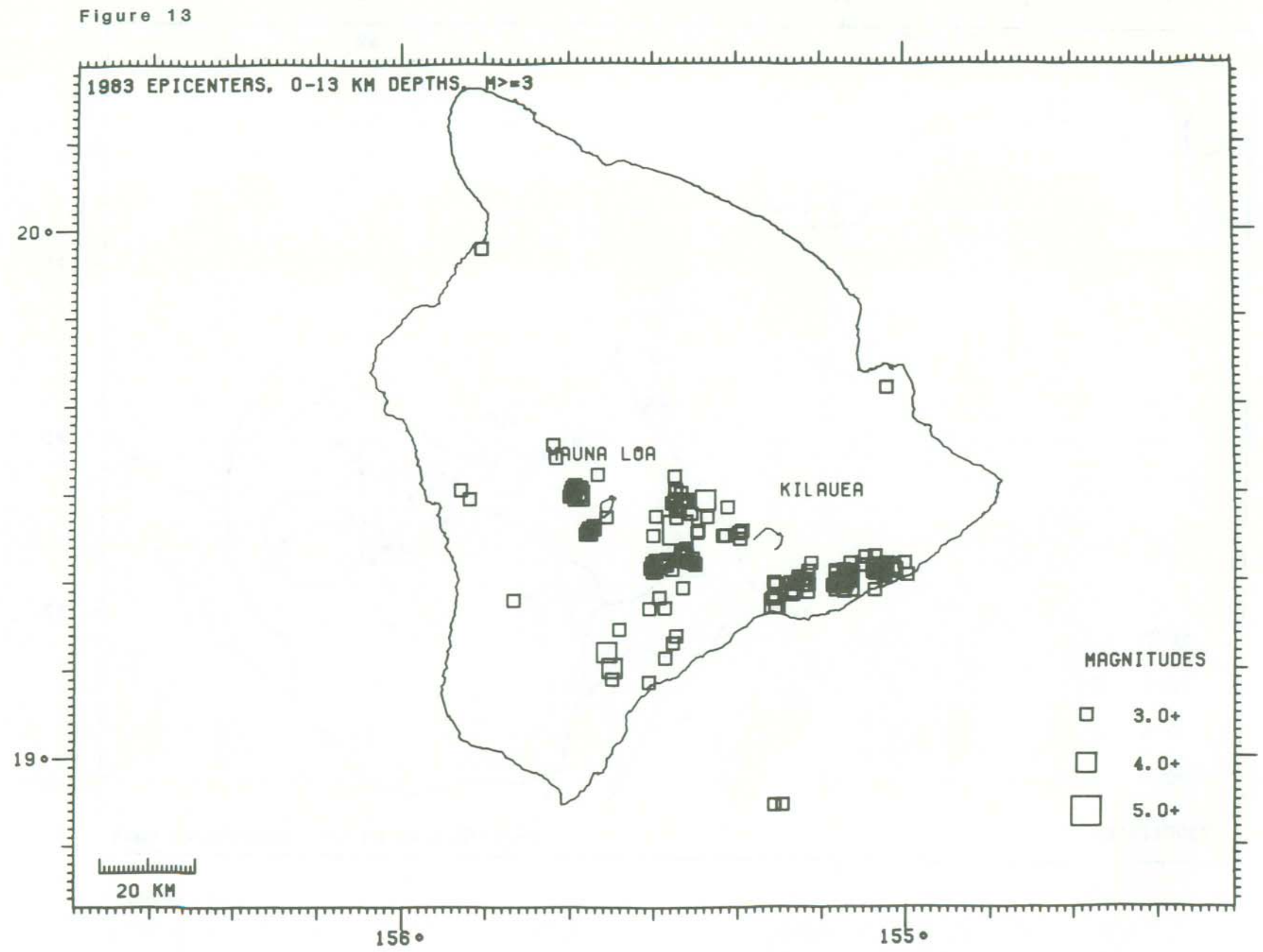


Figure 14

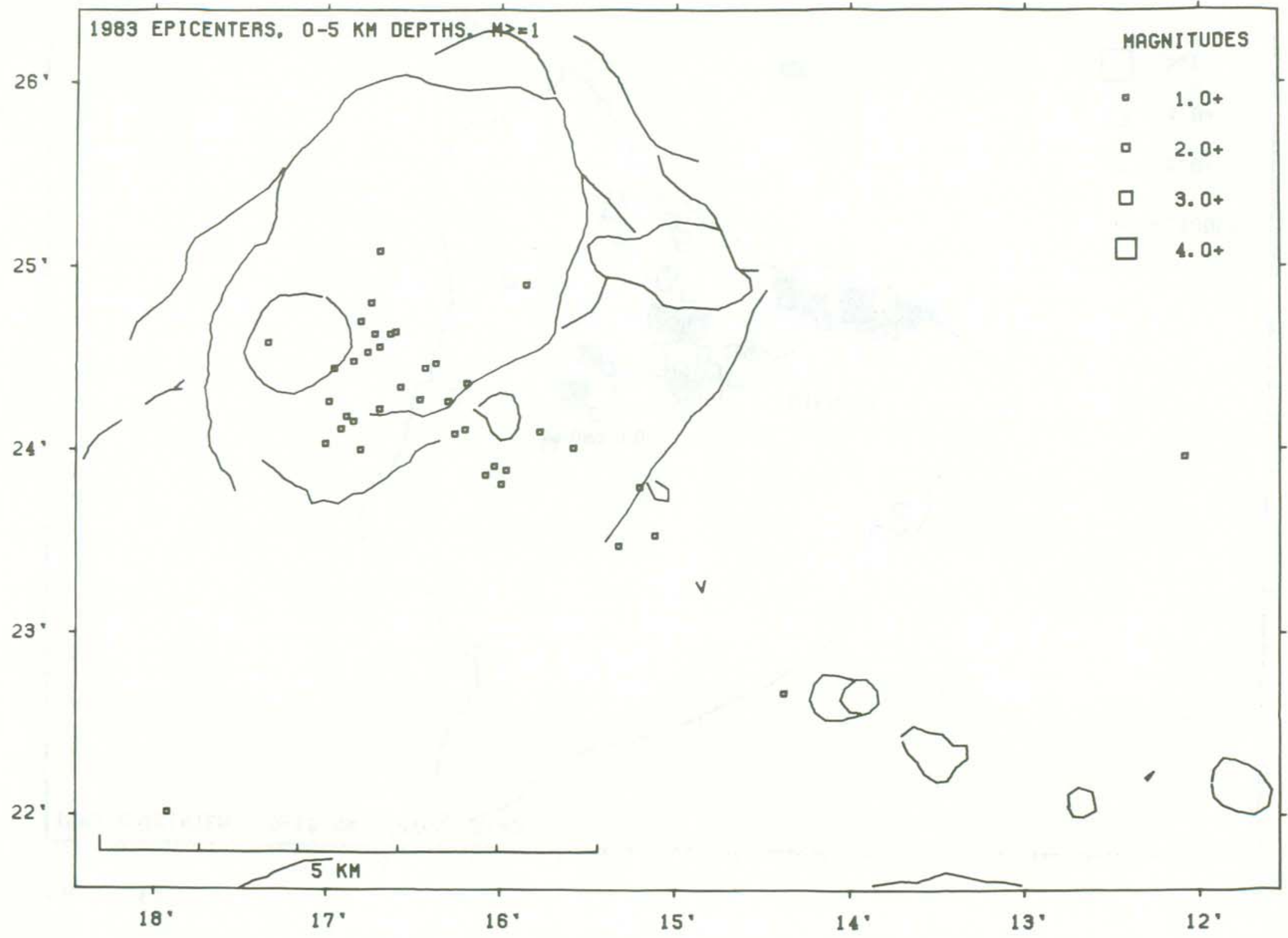


Table 5 is a chronological listing of successfully located earthquakes. For each event the following data are presented:

Origin time in Hawaiian Standard Time: date, hour (HR), minute (MN), and second (SEC).

Epicenter in degrees and minutes of north latitude (LAT N) and west 1ongitude (LON W).

DEPTH - Depth of focus in $\mathrm{km}$.

AMP MAG - Amplitude magnitude, if determined.

DUR MAG - Duration magnitude, if determined.

NR - Number of arrivals ( $P$ or $S$ ) used for solution.

NS - Number of S arrivals used for solution.

GAP DEG - Largest azimuthal separation in degrees between stations.

RMS SEC - Root mean square error of time residuals in sec.

$$
\mathrm{RMS}=\left(\sum \mathrm{R}_{\mathbf{j}}^{2} / \mathrm{NR}\right)^{1 / 2}
$$

MIN DIS - Epicentral distance in $\mathrm{km}$ to the third nearest station.

ERH km - Standard error of the epicenter in $\mathrm{km}$.

ERZ km - Standard error of depth of focus in $\mathrm{km}$.

REMK - Remarks, three letter code for geographic location of event. See Figure 2 for location of mnemonic code. Additional one letter codes have the following meanings:

$F$ - felt

L - long period character

$T$ - associated with harmonic tremor

B - quarry or other blast

* - the location program had a convergence problem, which usually means that the depth may be unreliable.

Table 5 lists all events located during 1983. Table 6 lists only events of magnitude 3.0 or larger. 


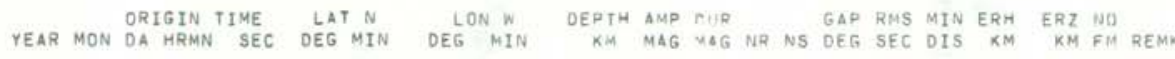

1983 JAN $11 \quad 452 \quad 14.66 \quad 1920.97 \quad 155 \quad 11.26$

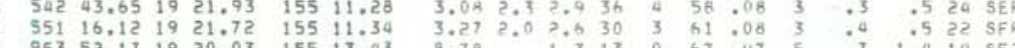

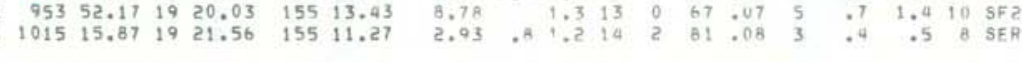

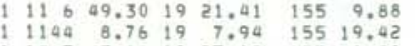
14055.19 19 $23.04 \quad 155$.

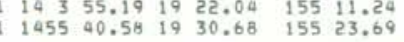
$1612 \quad 54.16 \quad 1921.83 \quad 155 \quad 11.30$

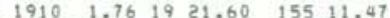
204618.181919 .59 155 8.10

$12236 \quad 35.48 \quad 1921.89 \quad 155 \quad 10.98$

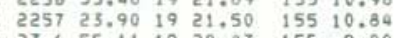

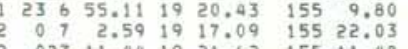
$025 \quad 38.16 \quad 19 \quad 22.00 \quad 155 \quad 11.37$
03350

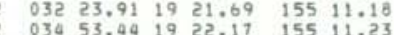

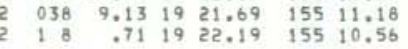
$\begin{array}{llllll}113 & 40.44 & 19 & 22.11 & 155 & 10.36\end{array}$

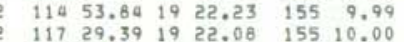

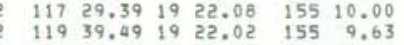

$\begin{array}{llllll}137 & 53.27 & 19 & 22.17 & 155 & 10.20\end{array}$

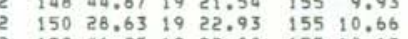

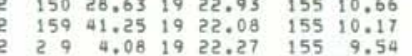

$\begin{array}{lllllll}2 & 214 & 58.50 & 19 & 22.05 & 155 & 10.01\end{array}$

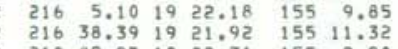

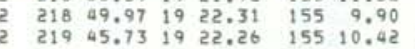

$\begin{array}{lllllll}2 & 220 & 14.67 & 19 & 22.11 & 155 & 9.61 \\ 2 & 226 & 18.45 & 19 & 22.22 & 155 & 9.84\end{array}$

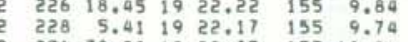

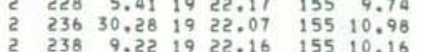
$\begin{array}{lllllll}240 & 39.51 & 19 & 22.61 & 155 & 9.96\end{array}$ $\begin{array}{lllllll}2 & 246 & 15.49 & 19 & 22.14 & 155 & 9.32\end{array}$

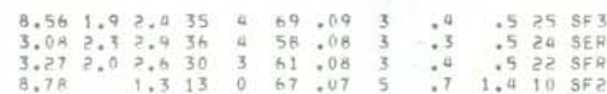

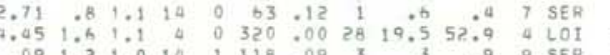

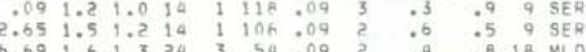
$\begin{array}{llllllllllll}2.56 & 1.5 & 1.6 & 16 & 1 & 112 & .10 & 3 & .5 & .5 & 7 \text { SEP }\end{array}$

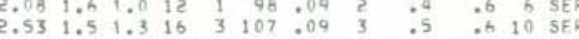

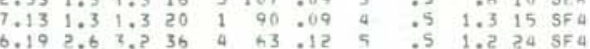
$\begin{array}{llllllllllll}2.53 & 1.5 & 1.0 & 15 & 2 & 102 & .11 & z & .6 & .4 & 10 & \text { SER }\end{array}$

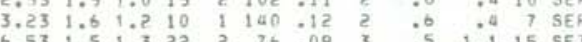

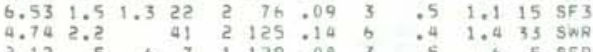

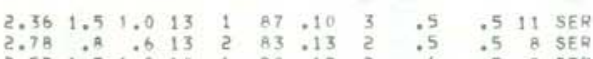

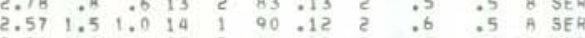

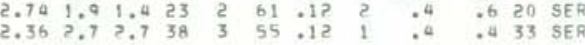
$\begin{array}{lllllllllll}2.70 & 1.7 & 1.5 & \text { 2h } & 5 & 92 & .09 & 1 & .5 & .3 & 21 \\ 3 & \text { SER }\end{array}$

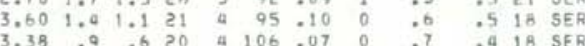

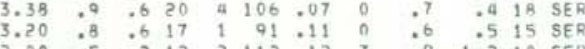

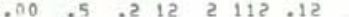

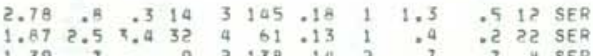

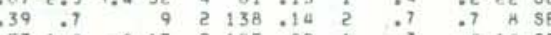

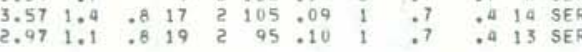
$\begin{array}{lllllllllllll}3.53 & .8 & .6 & 13 & 3 & 165 & .08 & 0 & 1.3 & .4 & 11 & \text { SER }\end{array}$

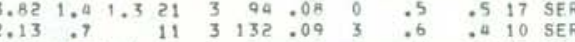
$\begin{array}{lllllllllllll}3.97 & 1.3 & 1.1 & 20 & 2 & 96 & 06 & 1 & .4 & .4 & 18 & \text { SER } \\ 3.57 & .9 & .9 & 14 & 3 & 99 & .09 & 1 & .6 & .4 & 13 & \text { SER }\end{array}$

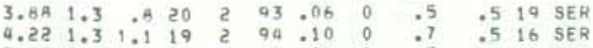

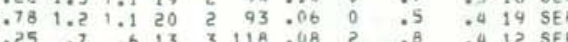

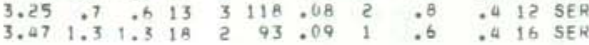

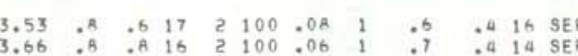

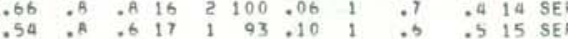

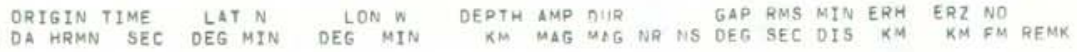

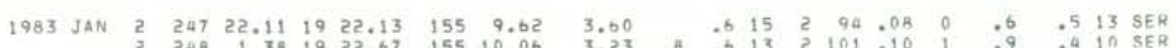

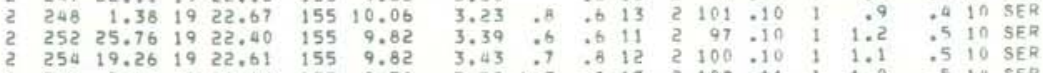

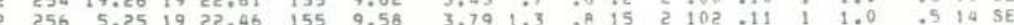

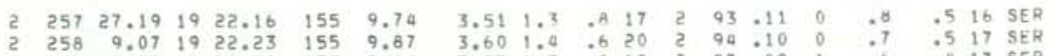

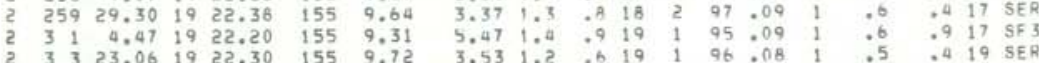

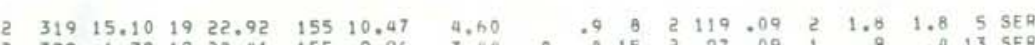

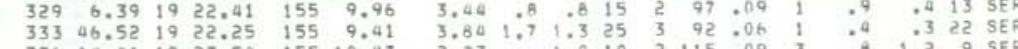

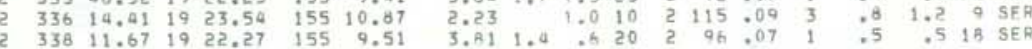
$\begin{array}{lllllllllllllllll}339 & .43 & 19 & 22.57 & 155 & 9.44 & 3.38 & 1.6 & 1.1 & 14 & 2 & 105 & .10 & 1 & .9 & .4 & 13\end{array}$ SER

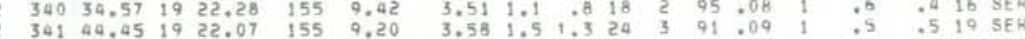

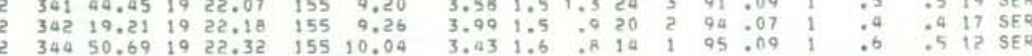
$35023.421922 .61 \quad 155 \quad 9.40$

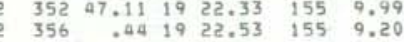

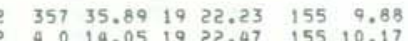

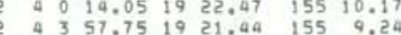
$3 \quad 4 \quad 6 \quad 45.981922 .39 \quad 155 \quad 9.80$

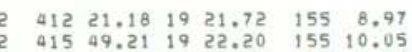
5 411 49.2119 21.

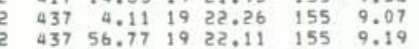

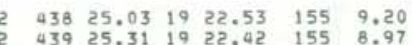

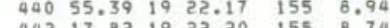

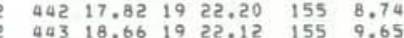

$\begin{array}{lllllll}2 & 446 & 17.62 & 19 & 22.15 & 155 & 9.73 \\ 2 & 450 & 17.93 & 19 & 22.22 & 155 & 9.60\end{array}$ $450 \quad 54.53$ 19 21.96 155 9.60

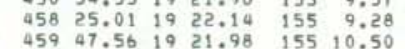

$\begin{array}{lllllll}5 & 0 & 21.56 & 19 & 22.82 & 155 & 10.24\end{array}$ $\begin{array}{llllll}516 & 15.00 & 19 & 22.32 & 155 & 10.25 \\ 518 & 27.05 & 19 & 20.33 & 155 & 9.58\end{array}$

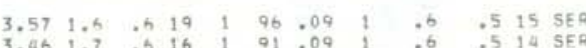

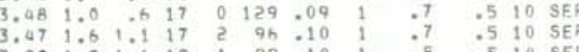

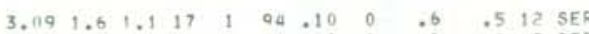

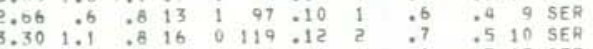

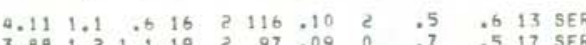
\begin{tabular}{lllllllllll}
\hline & 6 & 12 & 2 & 167 & 010 & 1 & 1.1 & .5 & 11 & SER
\end{tabular}

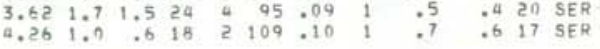

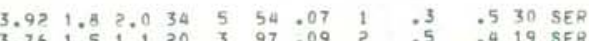

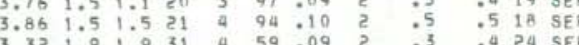

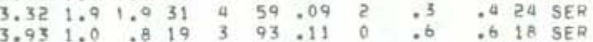
$\begin{array}{llllllllllll}3.33 & 1.5 & 1.3 & 19 & 0 & 93 & 11 & 0 & .5 & .4 & 18 & \text { SER }\end{array}$

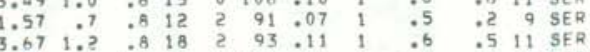
$\begin{array}{llllllll}5 & 0 & 04 & 04 & 1.4 & .5 & 5 & \text { SER }\end{array}$

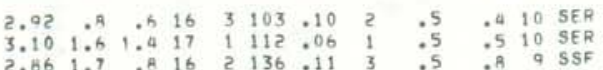




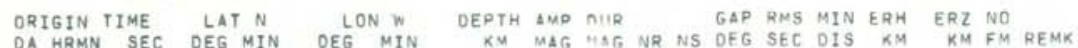

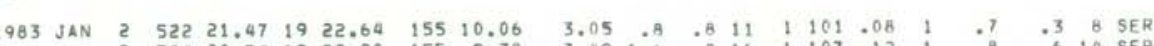

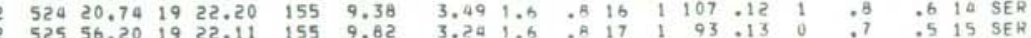

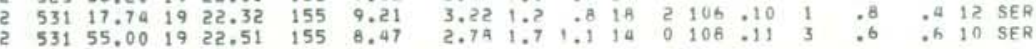

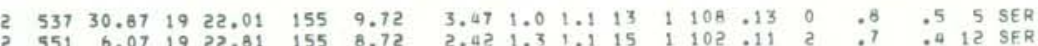

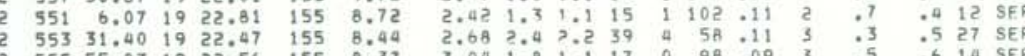

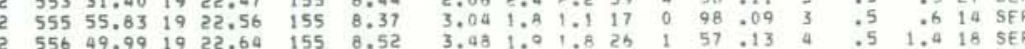

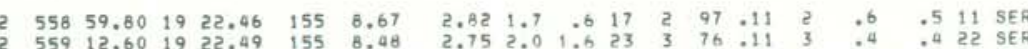

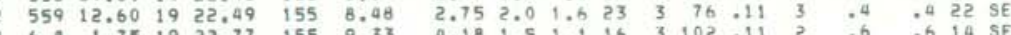

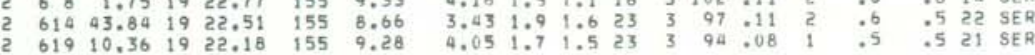

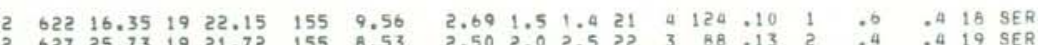

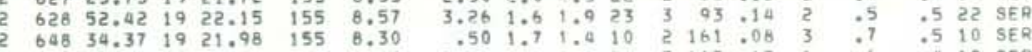

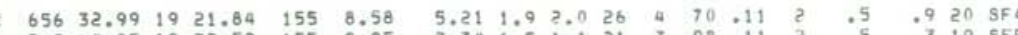

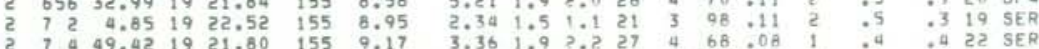

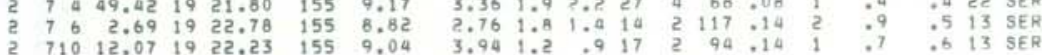

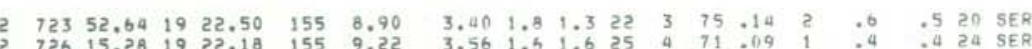

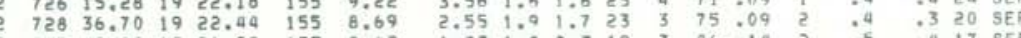

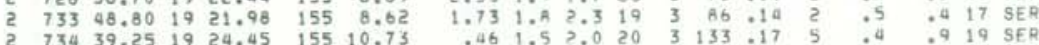

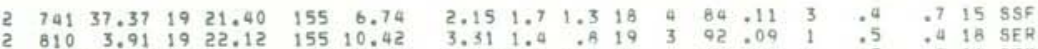

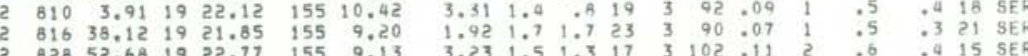

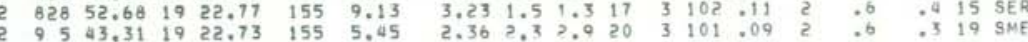

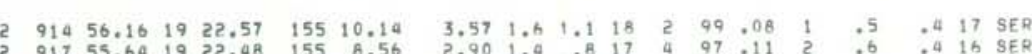

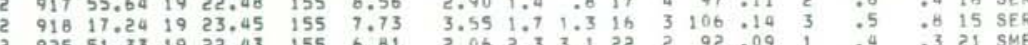

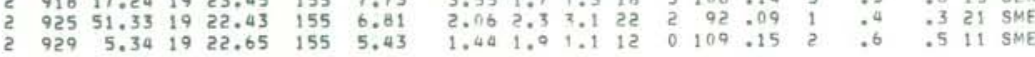

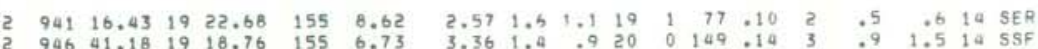

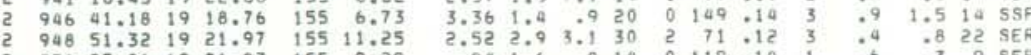

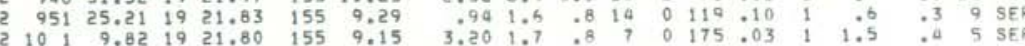

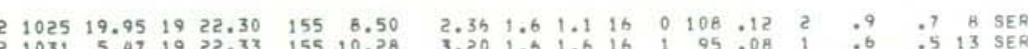

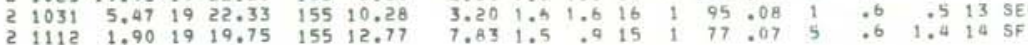

HVO EARTHQUAKE SUMMARY LIST

PAGE

ORIGIN TIME GAT NH YEAR MON DA HRMN SEC DEG MIN DEG MIN KM MAG YAG NR NS OEG SEC OIS KM KM FM REMK

$\begin{array}{llllllll}983 & \text { JAN } 2 & 1117 & 25.22 & 19 & 20.51 & 155 & 3.55\end{array}$ 2 114143.541920 .0515510 .84

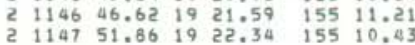

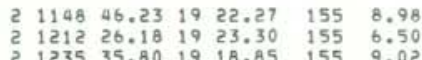
$\begin{array}{lllllll}2 & 1235 & 35.80 & 19 & 18.85 & 155 & 9.02 \\ 2 & 1240 & 28.37 & 19 & 16.99 & 155 & 5.96 \\ 2 & 1253 & 5.96 & 19 & 23.92 & 155 & 9.46\end{array}$ $\begin{array}{lllllll}\text { z } & 1257 & 3.24 & 19 & 21.88 & 155 & 10.9\end{array}$ 2 . 132013.80 1921. $\begin{array}{lllllll}2 & 1321 & 13.44 & 19 & 22.85 & 155 & 8.64 \\ 2 & 1330 & 58.75 & 19 & 22.47 & 155 & 8.92\end{array}$

$\begin{array}{llllll}2 & 1338 & 36.72 & 19 & 23.37 \quad 155 \quad 7.28\end{array}$

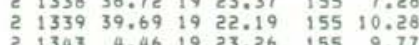
$\begin{array}{rrrrrrr}2 & 1343 & 4.46 & 19 & 23.26 & 155 & 9.7 \\ 2 & 1352 & 27.47 & 19 & 22.31 & 155 & 9.40\end{array}$

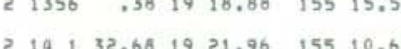
$\begin{array}{llllllll}2 & 14 & 1 & 32.68 & 19 & 21.96 & 155 & 10.62 \\ 2 & 14 & 9 & 47.92 & 19 & 22.41 & 155 & 10.67\end{array}$

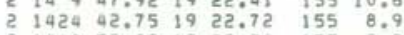

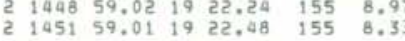
$\begin{array}{lllllll}2 & 1454 & 46.73 & 19 & 22.77 & 155 & 7.92 \\ 2 & 1458 & 9.37 & 19 & 22.00 & 155 & 9\end{array}$

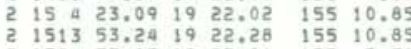
$2152433.03 \quad 1923,06 \quad 155,7,51$

$\begin{array}{rrrrrrr}2 & 1525 & 15.27 & 19 & 22.73 & 155 & 7.69 \\ 2 & 1532 & 40.01 & 19 & 23.96 & 155 & 12.09\end{array}$

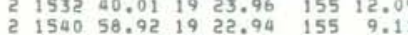
$\begin{array}{lllllll}2 & 1541 & 43.42 & 19 & 20.06 & 155 & 9.22 \\ 2 & 1550 & 11.22 & 19 & 22.86 & 155 & 7.52\end{array}$

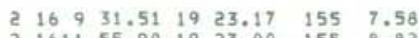

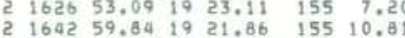

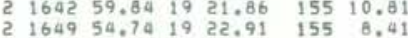

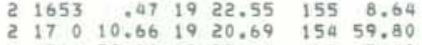
$\begin{array}{lllllll}2 & 1712 & 30.89 & 19 & 23.44 & 155 & 7.40 \\ 2 & 1719 & 53.90 & 19 & 22.71 & 155 & 8.77 \\ 2 & 1720 & 30.51 & 19 & 22.72 & 155 & 8.81\end{array}$ $\begin{array}{llllll}\text { c } 1726 & 51.16 & 19 & 22.73 & 155 & 8.67\end{array}$ $\begin{array}{lllllll}2 & 1726 & 51.16 & 1922.73 & 155 & 8.67 \\ 2 & 1733 & 29.60 & 19 & 23.10 & 155 & 7.55\end{array}$

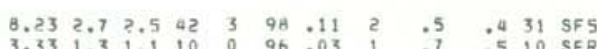

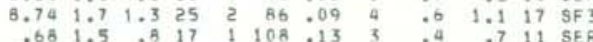

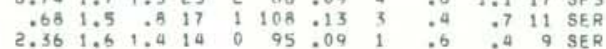

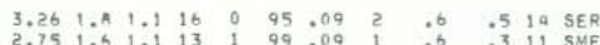

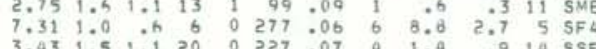

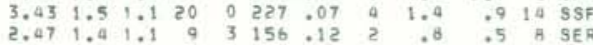
$\begin{array}{lllllllllllll}2.94 & 1.3 & .6 & 16 & 3 & 103 & 08 & 2 & .5 & .4 & 15 & \text { SER } \\ 7.19 & -4 & 0 & 35 & 9 & 176 & 0.10 & 3 & .5 & .7 & 30 & \text { SE }\end{array}$

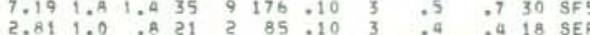

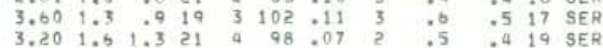
$\begin{array}{lllllllllllllll}4.21 & 1.9 & 1.1 & 19 & 2 & 103 & .10 & 2 & .5 & .7 & 17 & \text { SER }\end{array}$

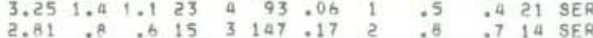

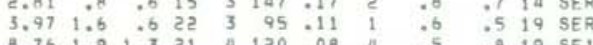
$\begin{array}{lllllllllllll}3.36 & 1.3 & .6 & 15 & 2 & 103 & 11 & 1 & .7 & .5 & 10 & \text { SER }\end{array}$

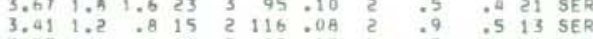

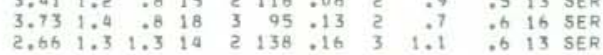

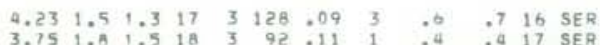

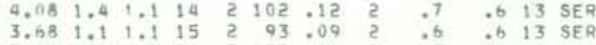

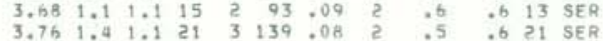

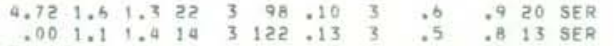
$3.501 .20 .4 \quad 16 \quad 33104.09 \quad 2 \quad: 6 \quad .5$ is SER

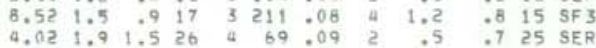

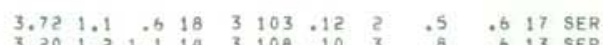

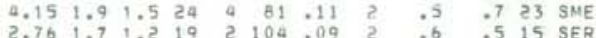

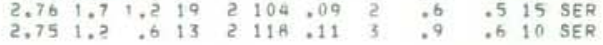

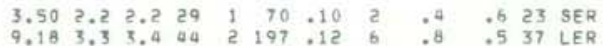

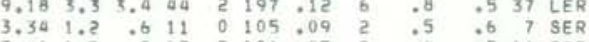

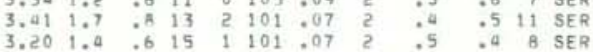

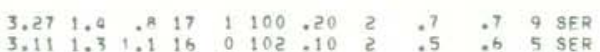


HVO EARTHQUAKE SUMMARY LIST

PAGE 5

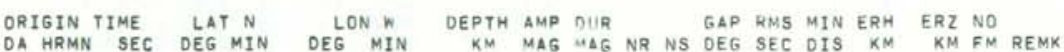

1983 JAN $2 \begin{array}{lllllllllllllllllll}18 & 3 & 29.65 & 19 & 23.46 & 155 & 6.99 & 2.34 & 1.9 & .8 & 10 & 0 & 103 & .09 & 2 & .6 & .3 & 6 & \text { SME }\end{array}$

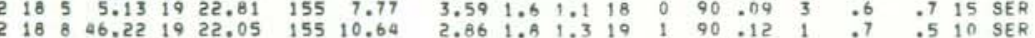

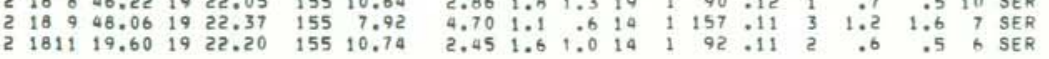
$\begin{array}{lllllllllllllllllll}2 & 1826 & 43.77 & 19 & 22.59 & 155 & 9.79 & 2.93 & 1.2 & .6 & 7 & 0 & 132 & .09 & 1 & 1.0 & .5 & 7 & \text { SER } \\ 2 & 1837 & 32.14 & 19 & 22.75 & 155 & 8.76 & 3.03 & 1.03 & .0 & 17 & 1 & 101 & .10 & 2 & .6 & .5 & 11 & \text { SER }\end{array}$

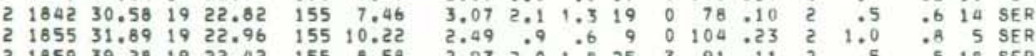
$\begin{array}{lllllllllllllllllll}2 & 19 & 2 & 15.18 & 19 & 22.59 & 155 & 8.83 & 3.02 & 1.9 & 1.6 & 24 & 2 & 94 & .07 & 2 & .4 & .4 & 19 \text { SER }\end{array}$

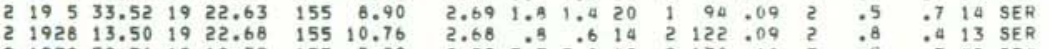

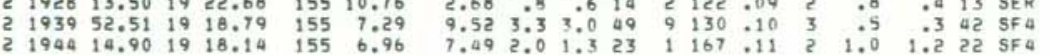

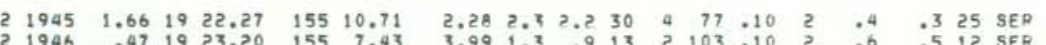

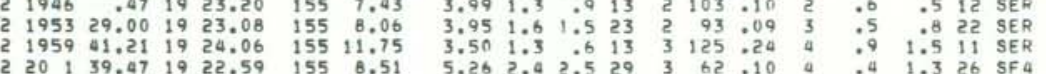

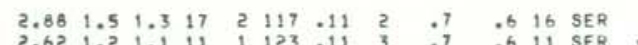

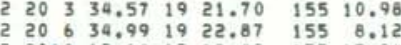

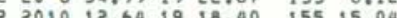
$\begin{array}{lrrrrrr}2 & 2011 & 14.53 & 19 & 21.82 & 155 & 10.68 \\ 2 & 2012 & 5.01 & 19 & 23.27 & 155 & 7.46\end{array}$

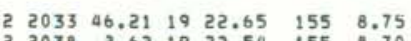

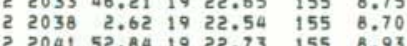

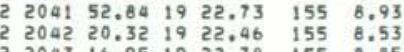

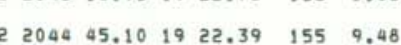

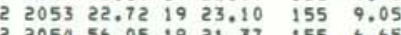

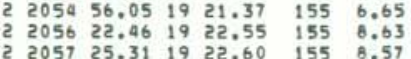
$\begin{array}{lllllll}2 & 2058 & 17.60 & 19 & 22.68 & 155 & 8.60 \\ 2 & 21 & 15.39 & 19 & 22.63 & 155 & 8.63\end{array}$ $\begin{array}{rrrrrrrr}2 & 21 & 4 & 30.45 & 19 & 22.91 & 155 & 8.73 \\ 2 & 21 & 9 & 11.47 & 19 & 17.60 & 155 & 12.62 \\ 2 & 21 & 9 & 46.06 & 19 & 22.47 & 155 & 6.41\end{array}$ $\begin{array}{lllllll}2 & 2113 & 7.36 & 19 & 24.77 & 155 & 8.97 \\ 5 & 2118 & 48.02 & 19 & 23.00 & 155 & 7.41\end{array}$ $\begin{array}{lllllll}2 & 2120 & 1.31 & 19 & 23.61 & 155 & 8.63 \\ 2 & 2140 & 21.69 & 19 & 22.82 & 155 & 7.66\end{array}$

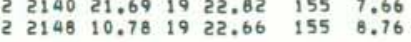
$\begin{array}{lllllll}2 & 2150 & 58.44 & 19 & 22.99 & 155 & 7.28\end{array}$

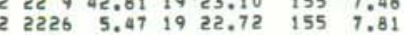

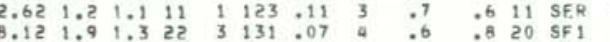
$\begin{array}{llllllllllllll}2.97 & 09 & .6 & 13 & 0 & 115 & .15 & 2 & .8 & .6 & 13 & \text { SER } \\ 3.91 & 1.4 & .6 & 20 & 3 & 103 & .09 & 2 & .5 & .6 & 19 & \text { SER }\end{array}$ $\begin{array}{llllllllllll}3.29 & 1.9 & 1.6 & 25 & 4 & 93 & .08 & 2 & .4 & .4 & 23 & \text { SER }\end{array}$

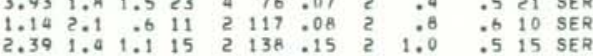
$\begin{array}{llllllllllll}2.99 & 1.8 & 1: 1 & 15 & 2 & 138 & 0.15 & 2 & 1.0 & .5 & 15 & \text { SER } \\ 2.98 & 1.0 & 19 & 3 & 101 & .07 & 2 & .5 & .3 & 17 & \text { SER }\end{array}$ $\begin{array}{lllllllllllllll}3.88 & 1.2 & .8 & 14 & 2 & 129 & .07 & 1 & .8 & .5 & 13 & \text { SER } \\ 3.32 & : 7 & 0.0 & 17 & 3 & 106 & 00 & 2 & .7 & .6 & 16 & \text { SER }\end{array}$

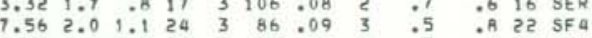

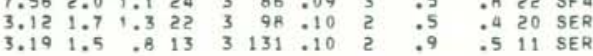
$\begin{array}{rrrrrrrrrrrrr}3.41 & 1.4 & 1.1 & 13 & 0 & 100 & 06 & 3 & .5 & .4 & 5 & \text { SER } \\ 3.26 & 2.0 & 1.1 & 18 & 0 & 93 & .05 & 2 & .4 & .5 & 14 & \text { SER }\end{array}$ $\begin{array}{llllllllllll}2.90 & 1.5 & 1.1 & 19 & 1 & 103 & 08 & 3 & .5 & .5 & 14 & \text { SER } \\ 4.77 & 1.5 & .6 & 18 & 0 & 142 & .08 & 2 & .6 & 1.1 & 9 & \text { SSER }\end{array}$

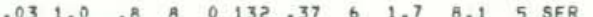

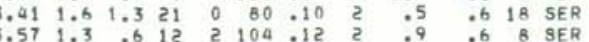

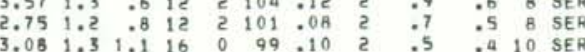

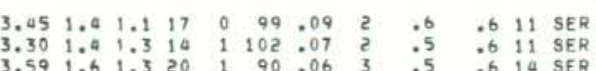

HVO EARTHQUAKE SUMMARY LIST

PAgE 6

ORIGIN TIME
DA LAT N 1983 JAN $22230 \quad 13.99 \quad 1922.88 \quad 155 \quad 8.48$

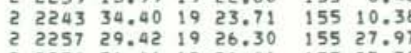
55.38

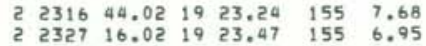
$\begin{array}{rrrrrrr}2 & 2333 & 3.51 & 19 & 23.56 & 155 & 6.89 \\ 2 & 2337 & 16.91 & 19 & 19.13 & 155 & 13.72\end{array}$ $\begin{array}{llllll}2352 & 22.72 & 19 & 19.53 & 155 & 8.28\end{array}$

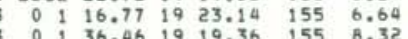

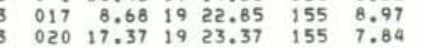

$\begin{array}{llllllll}3 & 1 & 5 & 41.75 & 19 & 24.44 & 155 & 16.46\end{array}$

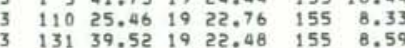
143
$145 \quad 36.69$
19 $\begin{array}{lllllll}3 & 159 & 13.17 & 19 & 23.64 & 155 & 7.05\end{array}$

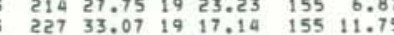
$\begin{array}{lrrrrrrr}3 & 231 & 4.06 & 19 & 21.91 & 155 & .36 \\ 3 & 315 & .95 & 19 & 22.81 & 155 & 7.74\end{array}$ $\begin{array}{lllllll}3 & 319 & 22.82 & 19 & 24.53 & 155 & 16.35\end{array}$

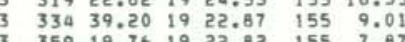
$\begin{array}{llllll}359 & 19.76 & 1922.82 & 155 & 7.87 \\ 4.6 & 29.66 & 19 & 24.27 & 155 & 17.19\end{array}$

$\begin{array}{llllll}512 & 14.30 & 19 & 24.15 & 155 & 16.85\end{array}$

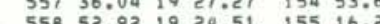

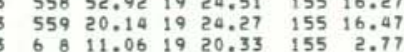

$\begin{array}{lllllll}3 & 627 & 10.47 & 19 & 22.59 & 155 & 9.00\end{array}$

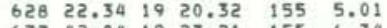

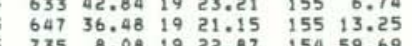
$\begin{array}{lllllll}3 & 745 & 44.77 & 19 & 22.99 & 155 & 2.27 \\ 3 & 837 & 10.06 & 19 & 19.71 & 155 & 8.50\end{array}$

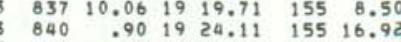
$\begin{array}{rrrrrrr}3 & 840 & .90 & 19 & 24.11 & 155 & 16.92 \\ 3 & 910 & 5.44 & 19 & 24.46 & 155 & 16.78 \\ 3 & 10 & 8 & 22.64 & 19 & 23.61 & 155 \\ 4\end{array}$

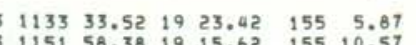

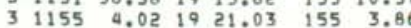

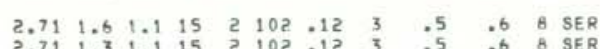
$\begin{array}{llllllllllll}.10 & .5 & 5 & 0 & 118 & .05 & 3 & .8 & 2.2 & 4 & \text { SER } \\ .08 & 2.6 & 2.3 & 45 & 9 & 37 & 0.13 & 5 & .3 & .7 & 31 & \text { KAO }\end{array}$ $\begin{array}{rrrrrrrrrrrr}3.42 & 1.9 & 1.9 & 22 & 2 & 92 & .09 & 3 & .4 & .6 & 19 & \text { SER } \\ 2.78 & 1.4 & 1.3 & 15 & 4 & 103 & .10 & 2 & : 5 & .3 & 14 & \text { SME }\end{array}$

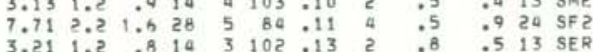

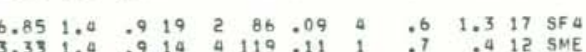
$\begin{array}{llllllllllllll}23 & 1.6 & 1.4 & 20 & 1 & 87 & .11 & 4 & .8 & 1.3 & 20 & \text { SF }\end{array}$ $\begin{array}{llllllllllll}3.55 & 1.1 & 0 & 16 & 3 & 103 & .07 & 2 & .6 & .5 & 15 & \text { SER } \\ .98 & 1.4 & 0 & 17 & 3 & 106 & .11 & 3 & .5 & .6 & 11 & \text { SER }\end{array}$ $\begin{array}{llllllllllllll}3.16 & 1.0 & 1.0 & 12 & 2 & 131 & .07 & 1 & .6 & .3 & 10 & \text { SEC }\end{array}$

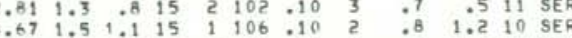

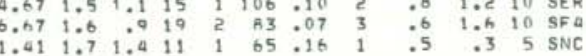
$\begin{array}{llllllllllll}2.50 & 1.3 & .8 & 11 & 1 & 106 & .09 & 2 & .6 & .5 & \text { S SME }\end{array}$

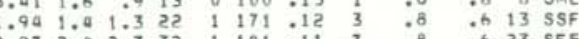

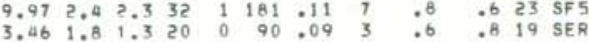

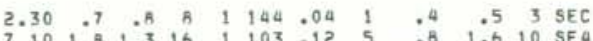

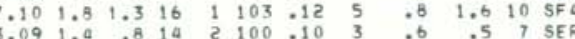

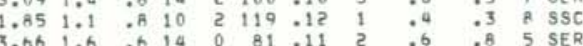

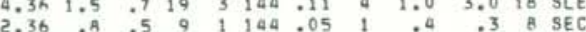

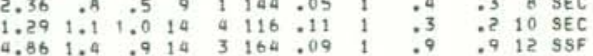

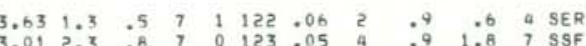
$\begin{array}{lllllllllllll}3.15 & 1.6 & .9 & 12 & 1 & 99 & .09 & 1 & .5 & .6 & 8 & \text { SME } \\ 90 & 1.6 & 1.0 & 7 & 0 & 200 & 0.0 B & 3 & 3.0 & 4.1 & 5 & \text { DEP }\end{array}$

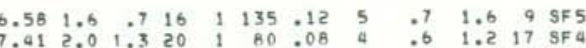

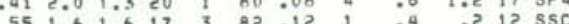

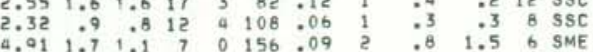

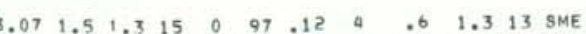

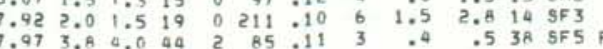
$\begin{array}{lllllllllllll}4.57 & 1.5 & 1.1 & 9 & 3 & 89 & 11 & 1 & .6 & .7 & \text { A } & \text { SSC }\end{array}$ 
HVO EARTHQUAKE SUMMARY LIST

PAGE, ORIGIN TIME LATN
DA HRMN SON

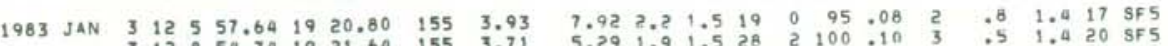

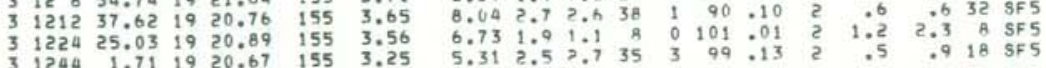

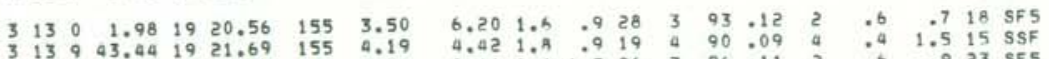

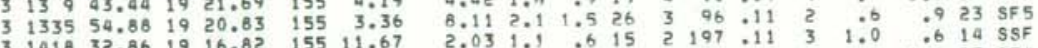

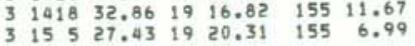
$\begin{array}{lllllll}3 & 1542 & 6.63 & 19 & 21.53 & 155 & 1.84\end{array}$ $3161553.16 \quad 1924.31 \quad 155 \quad 4.69$

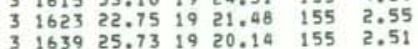

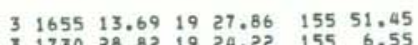

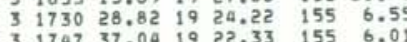

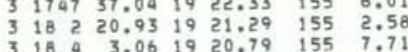
$\begin{array}{lllllll}3 & 1817 & 46.77 & 19 & 23.52 & 155 & 5.85\end{array}$

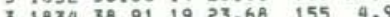

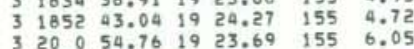
$\begin{array}{lllllll}3 & 2046 \quad 3.58 & 19 & 21.17 \quad 155 & 18.37\end{array}$ $32056 \quad 52.41 \quad 19 \quad 19.59 \quad 15513.81$

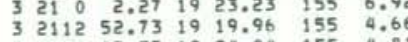
$\begin{array}{lllllll}3 & 2132 & 35.00 & 19 & 23.85 & 155 & 5.10\end{array}$ $\begin{array}{lllllll}3 & 2132 & 35.00 & 19 & 23.85 & 155 & 5.10 \\ 3 & 2137 & 51.94 & 19 & 24.70 & 155 & 5.37\end{array}$

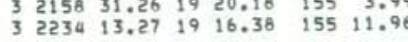
$\begin{array}{lllllll}3 & 2241 & 7.48 & 19 & 21.88 & 155 & 2.31\end{array}$ $3233456.901923 .91 \quad 155 \quad 5.10$

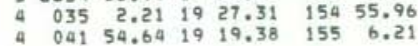
$\begin{array}{lllllll}4 & 046 & 31.81 & 19 & 19.06 & 155 & 10.30 \\ 4 & 128 & 15.44 & 19 & 20.06 & 155 & 7.73\end{array}$

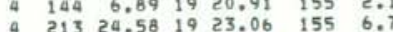
$4 \quad 216 \quad 16.98 \quad 19 \quad 19.19 \quad 15459.87$

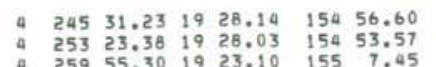

HVO EARTHQUAKE SUMMARY LIST

PAGE

PRIGIN TIME LAT N LON W DEPTH AMP PUR GAP RMS MIN ERH ERZ NO

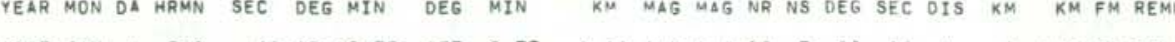

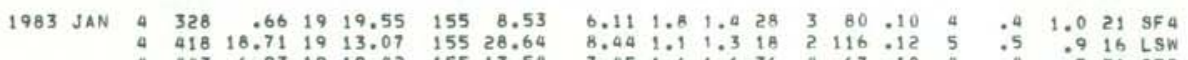

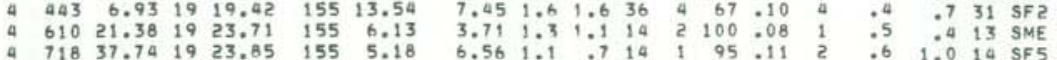
\begin{tabular}{l}
4 \\
\hline
\end{tabular}

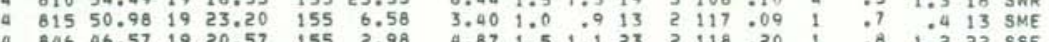

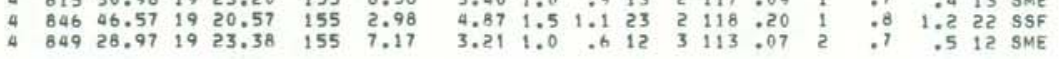

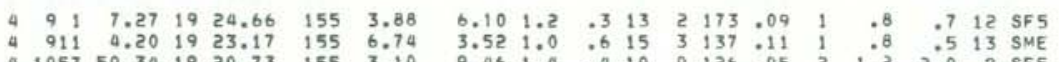

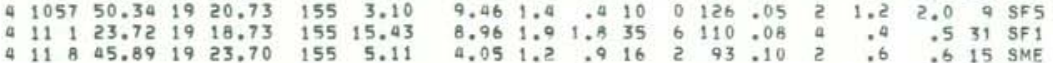

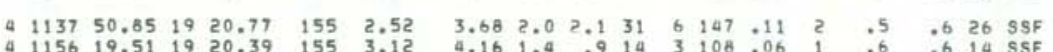

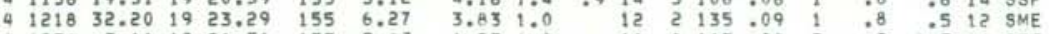

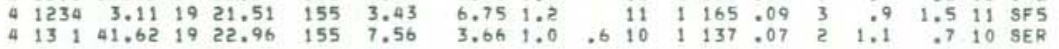

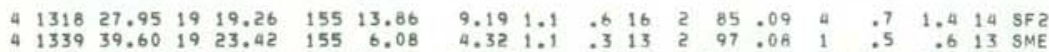

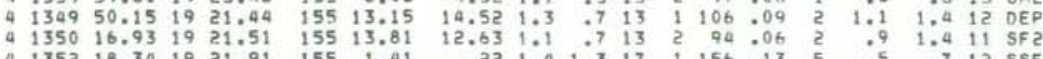

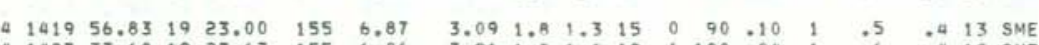

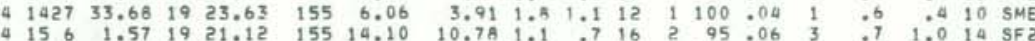

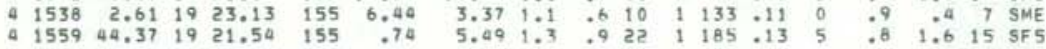

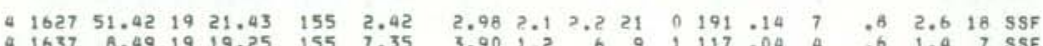

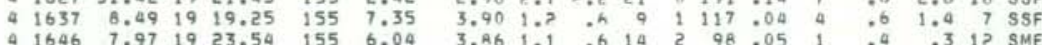

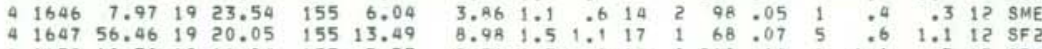

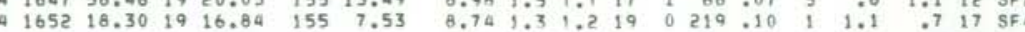

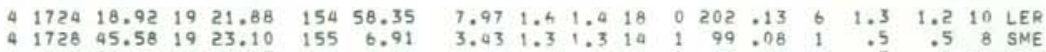

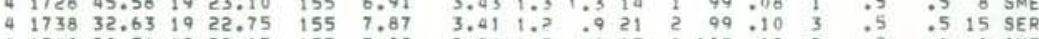

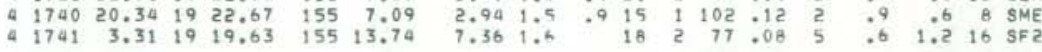
$\begin{array}{llllllllllllllllll}1741 & 16.83 & 19 & 20.05 & 155 & 13.73 & 7.77 & 1.5 & 1.1 & 14 & 2 & 71.07 & 5 & .6 & 1.3 & 11 & \mathrm{SF} & \end{array}$

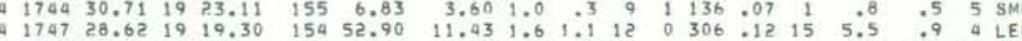

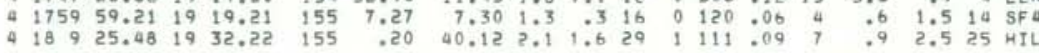

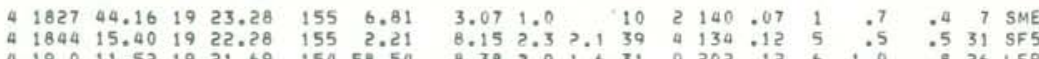




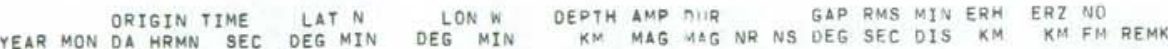

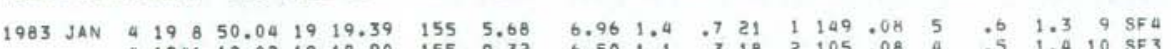

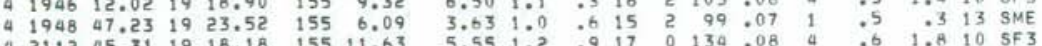

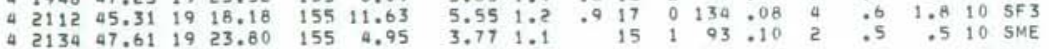

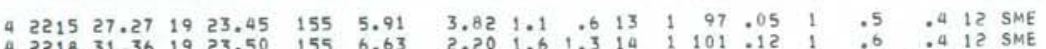

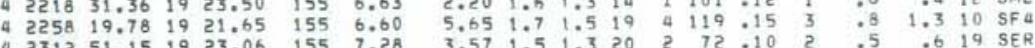

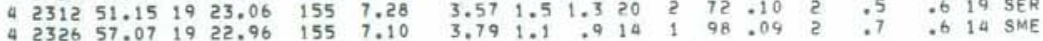
$\begin{array}{llllllllllllllllllll}5 & 011 & .91 & 19 & 23.82 & 155 & 6.65 & 3.72 & 1.0 & .3 & 15 & 2 & 106 & .06 & 2 & .5 & .5 & 14 & \text { SME } \\ 5 & 023 & 6.11 & 19 & 23.01 & 155 & 5.98 & 4.27 & 1.0 & & 13 & 2 & 119 & .10 & 1 & .9 & .6 & 11 & \text { SME }\end{array}$

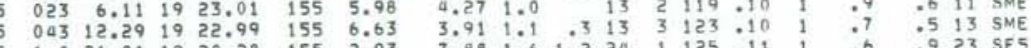

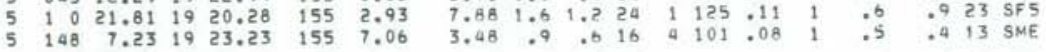

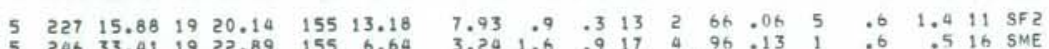

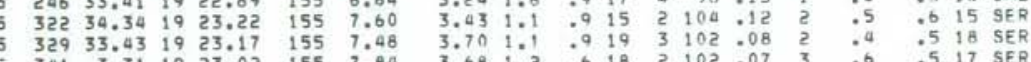

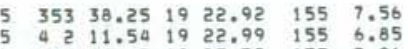

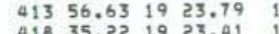

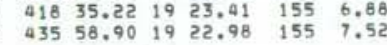

$\begin{array}{llllll}5 & 443 & 44.58 & 19 & 25.58 \quad 155 \quad 24.88\end{array}$

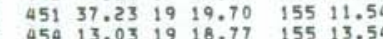
550635.6219 19.04 15513.56 $\begin{array}{lllllll}5 & 512 & 40.93 & 19 & 23.15 & 155 & 6.91 \\ 5 & 515 & 43.37 & 19 & 23.19 & 155 & 7.59\end{array}$ 528.7719 .930 .18 $54141.63 \quad 1922.98 \quad 155 \quad 7.40$ $\begin{array}{lllllll}545 & 26.94 & 19 & 23.47 & 155 & 7.51\end{array}$ $\begin{array}{llllll}557 & 0.96 & 19 & 22.94 & 155 & 7.17\end{array}$

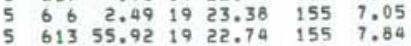
$\begin{array}{lllllll}5 & 616 & 59.27 & 19 & 23.00 & 155 & 7.34 \\ 5 & 620 & 12.99 & 19 & 23.02 & 155 & 7.03\end{array}$

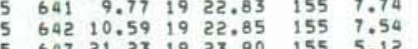
$\begin{array}{lllllll}5 & 655 & 10.31 & 19 & 23.37 & 155 & 7.85\end{array}$ $7110^{2} .641923 .03 \quad 155 \quad 7.36$

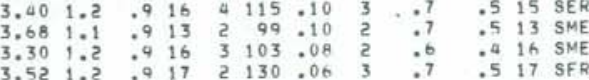

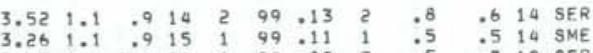

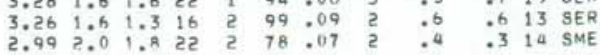

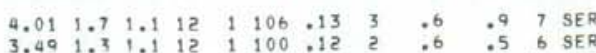

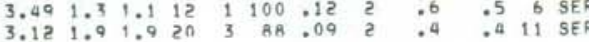
YEAR MON ORIGIN TIME HRMN SEC DET MIN DEG MIN OEPTH AMP MIIR NR NSAP RMS MIN ERH ERZ NO $\begin{array}{llllllllllllllllllll}1983 & \text { JAN } 5 & 729 & 33.75 & 19 & 20.80 & 155 & 2.97 & 5.56 & 2.5 & 2.3 & 31 & 1 & 119 & .13 & 2 & .5 & .9 & 26 & \text { SF } 5\end{array}$

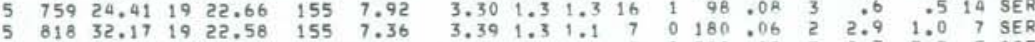

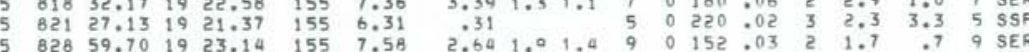

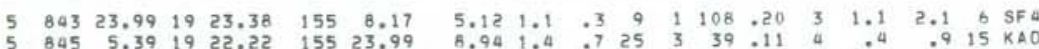

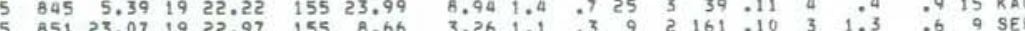

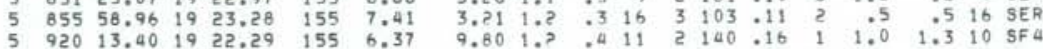

$\begin{array}{llllllllllllllllllll}5 & 926 & 27.07 & 19 & 12.89 & 155 & 2.51 & 19.77 & 2.4 & 1.7 & 18 & 1 & 297 & .16 & 20 & 4.7 & 3.2 & 18 & \text { DEP }\end{array}$ $\begin{array}{llllllllllllll} & & & \end{array}$

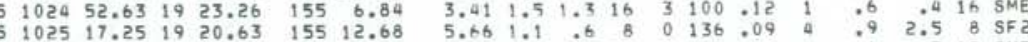
$5 \quad 1037 \quad 42.70 \quad 1923.54 \quad 155 \quad 6.68$ $\begin{array}{lllllll}5 & 1052 & 43.30 & 19 & 22.82 & 155 & 7.84 \\ 5 & 1053 & 26.90 & 19 & 18.69 & 155 & 13.30\end{array}$

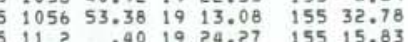
$\begin{array}{llllllll}5 & 11 & 63.67 & 19 & 22.80 & 155 & 7.26\end{array}$ $51130 \quad 35.741919 .41 \quad 15513.16$ $5113855.051918 .73 \quad 15513.50$ $\begin{array}{lllllll}5 & 1144 & 45.72 & 19 & 24.36 & 155 & 16.20 \\ 5 & 1145 & 45.96 & 19 & 18.82 & 155 & 13.75\end{array}$

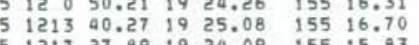
$5121727.891924 .09 \quad 155$

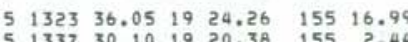

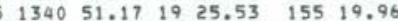
5134222.631924 .11 155 16.21 $134738.581920 .16 \quad 155$ $5 \quad 1354 \quad 38.00 \quad 19$
$23.67 \quad 155 \quad 7.28$

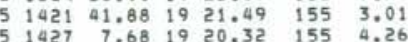

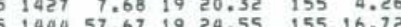
5 1446 $46,10 \quad 1924,56 \quad 155,16.79$

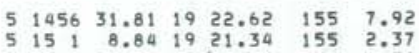

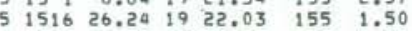

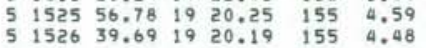
$\begin{array}{lllllll}5 & 1616 & 2.17 & 19 & 23.48 & 155 & 15.33\end{array}$

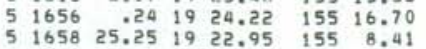

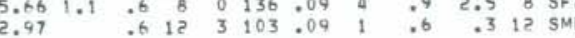

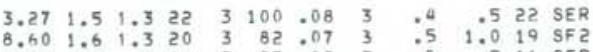

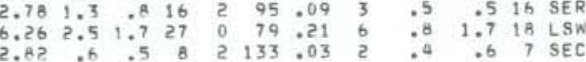

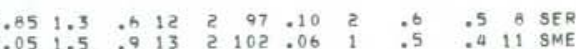

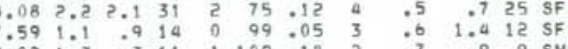

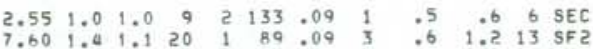

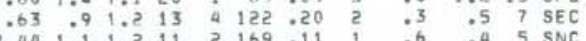

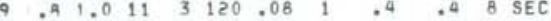
$\begin{array}{lllllllllllllll}1.99 & 1.4 & 1.2 & 17 & 4 & 80 & 07 & 1 & .3 & .2 & 10 & \text { SSC } \\ 6.08 & 2.9 & 2.7 & 34 & 2 & 170 & 071 & 1 & : 5 & 0 & 27 & \text { SF5 }\end{array}$ $\begin{array}{llllllllll}3.03 & 1.2 & 9 & 1 & 186 & -10 & 4 & 1.6 & 1.3 & 3 \\ \text { KAO }\end{array}$

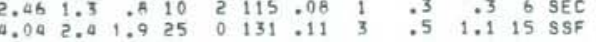
$\begin{array}{llllllllllll}4.91 & 1.3 & 10 & 0 & 107 & 13 & 2 & .9 & 1.6 & 4 & \text { SER } \\ 1.51 & 1.5 & 13 & 0 & 123 & .09 & 3 & : 4 & .9 & 7 & \text { SSF }\end{array}$

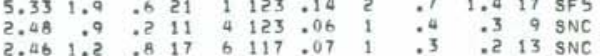




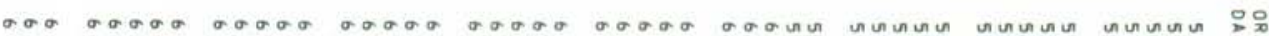

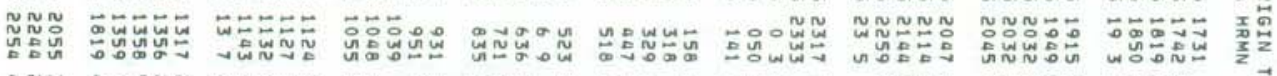

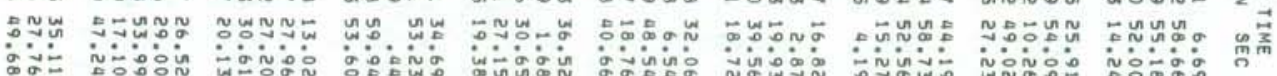

50.

บ

क्ष

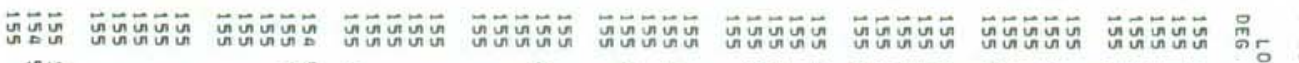

ب.

பñ

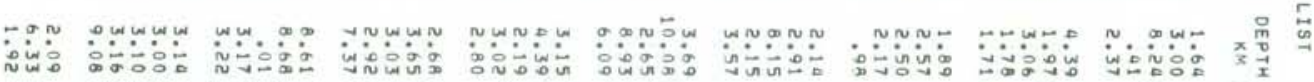

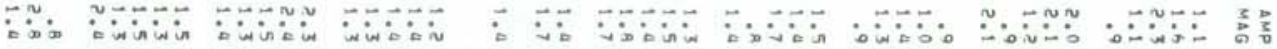

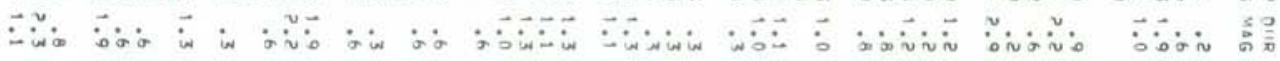

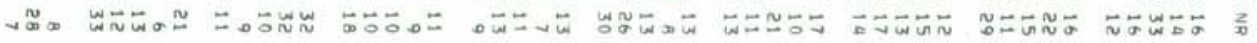

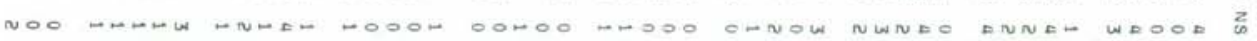

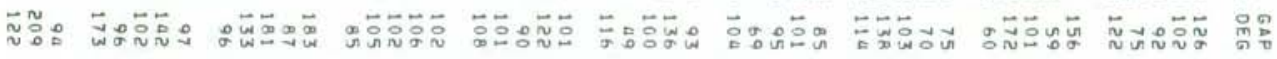

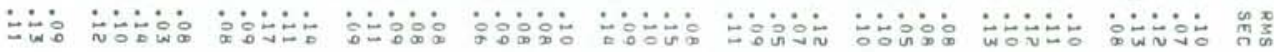

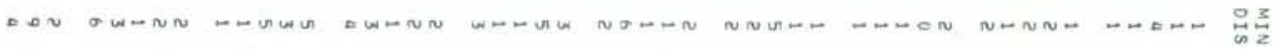

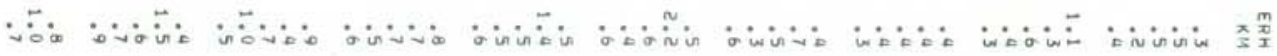

可

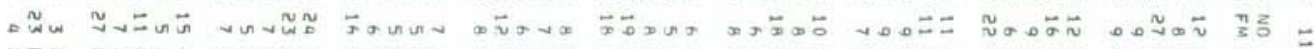

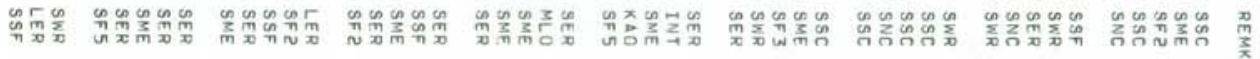

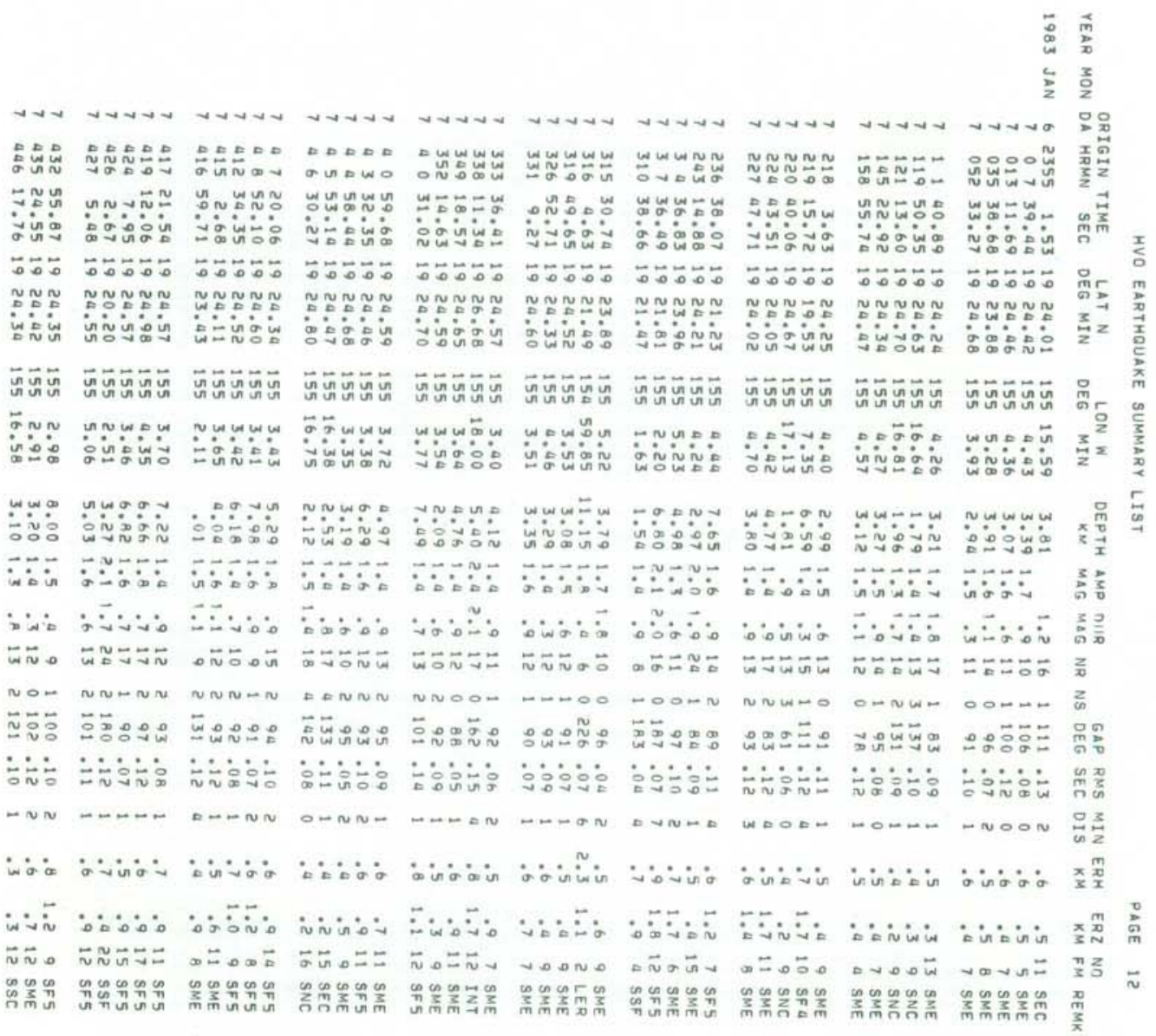




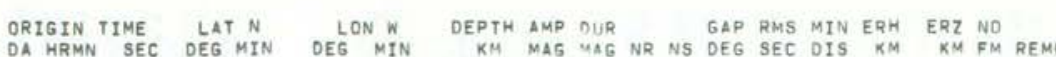
1983 JAN $7 \begin{array}{llllllllllllllllllllll}453 & 33.87 & 19 & 23.89 & 155 & 15.97 & 3.08 & 1.9 & 1.4 & 20 & 4 & 106 & .12 & 1 & .4 & .3 & 18 & \text { SEC }\end{array}$

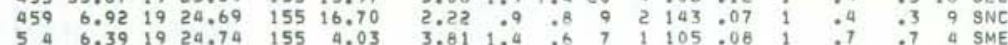

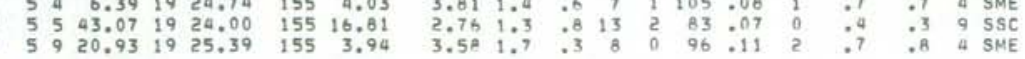
$511 \quad 52.81 \quad 1925.57 \quad 155 \quad 3.07$

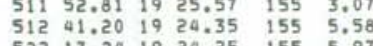

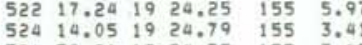
$52458.96 \quad 1924,73 \quad 155 \quad 3.67$

$\begin{array}{rlllll}527 & 14.89 & 19 & 24.74 & 155 & 3.06 \\ 530 & 16.72 & 19 & 24.74 & 155 & 3.24\end{array}$ $53713.801924 .66 \quad 155 \quad 4.00$

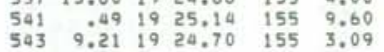

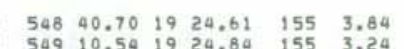

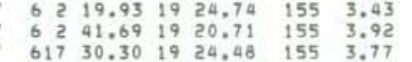

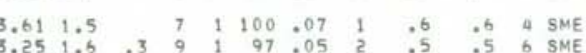

$\begin{array}{llllll}621 & 53.50 & 19 & 24.58 & 155 & 4.10 \\ 631 & 33.97 & 19 & 24.79 & 155 & 3.10\end{array}$

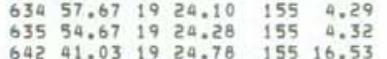

$653 \quad 34.371924 .46 \quad 155 \quad 4.36$
777 $72014.18 \quad 1924.44 \quad 155 \quad 16.96$

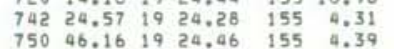

\begin{tabular}{llllll}
758 & 3.18 & 19 & 24.64 & 155 & 4.81 \\
\hline
\end{tabular} $827 \quad 44.991924 .51 \quad 155 \quad 4.53$ $\begin{array}{rrrrrr}829 & 8.21 & 19 & 23.53 & 155 & 4.01 \\ 834 & 51.92 & 19 & 24.63 & 155 & 16.73\end{array}$

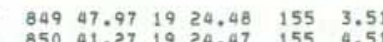
$94 \quad 30.391924 .53 \quad 155 \quad 16.77$

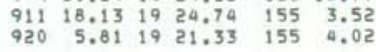

$\begin{array}{llllll}923 & 49.25 & 19 & 24.52 & 155 & 4.53 \\ 925 & 23.99 & 1924 & 243 & 155 & 4.35\end{array}$

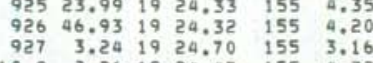

$\begin{array}{llllll}109 & 2.26 & 19 & 24.45 & 155 & 4.32 \\ 1010 & 28.33 & 19 & 24.37 & 155 & 4.61\end{array}$

$\begin{array}{lllllll}7 & 1010 & 28.33 & 19 & 24.37 & 155 & 4.61 \\ 7 & 1014 & 40.68 & 19 & 20.48 & 155 & 3.45 \\ 7 & 1017 & 37.41 & 19 & 25.39 & 155 & 3.12\end{array}$ $\begin{array}{llllllllllllll}.43 & 1.5 & .3 & 7 & 0 & 132 & 07 & 3 & .5 & 1.0 & 3 & \text { SME }\end{array}$

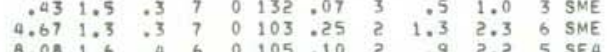

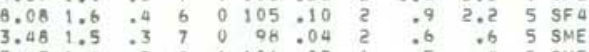

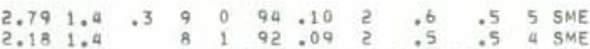

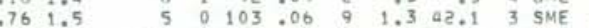

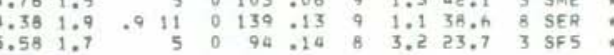

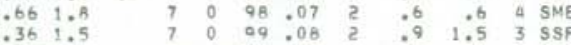

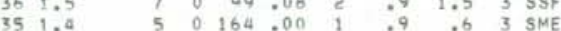

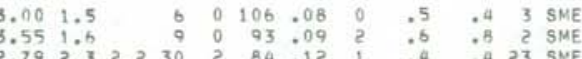

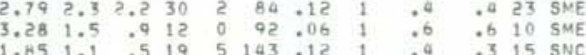

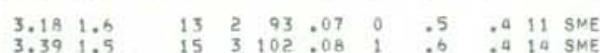

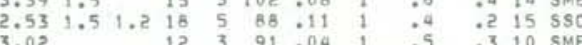
$\begin{array}{lllllllllllll}3.02 & & 12 & 3 & 91 & .04 & 1 & .5 & .5 & .3 & 15 & \text { SME } \\ 3.35 & 1.6 & .3 & 16 & 3 & 93 & .07 & 0 & .5 & .3 & 13 & \text { SME }\end{array}$

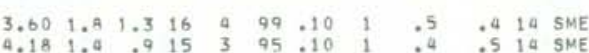
$\begin{array}{llllllllllll}2.42 & 1.8 & .6 & 13 & 3 & 92 & -10 & 2 & 04 & .4 & 11 & \text { SME }\end{array}$

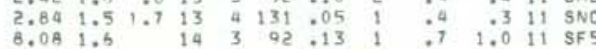

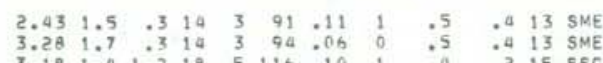

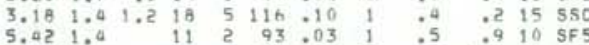

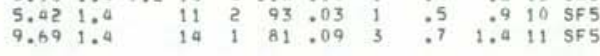

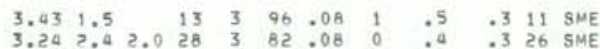

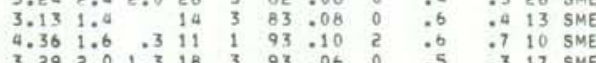
$\begin{array}{llllllllllll}3.68 & 1.4 & .6 & 15 & 3 & 95 & 06 & 1 & .5 & .4 & 14 & \text { SME }\end{array}$

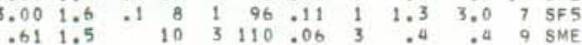

ORIGIN TIME LAT N LON W DEPTH AMP OIIR GAP RMS MIN ERH ERZ NO

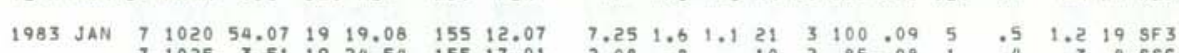
$\begin{array}{r}7 \\ 1026 \\ \hline\end{array}$

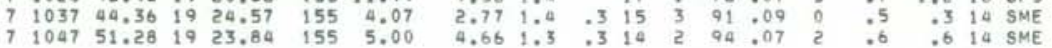

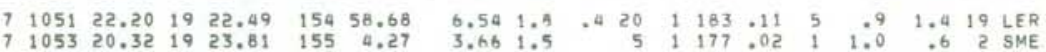

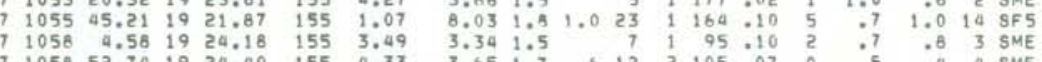

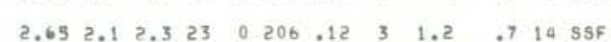

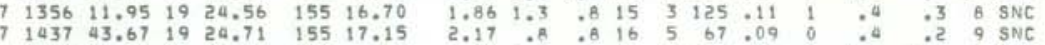

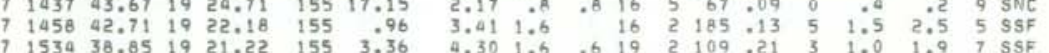

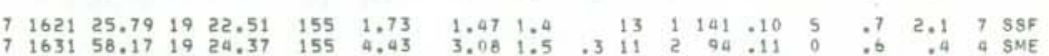

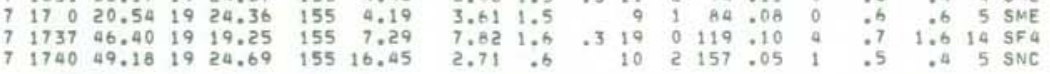

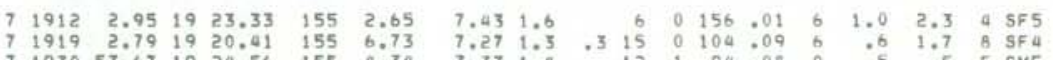

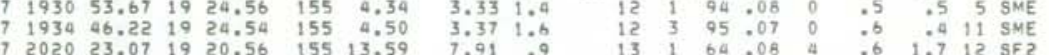

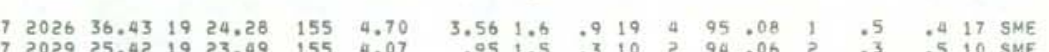

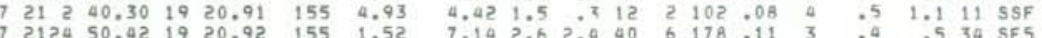

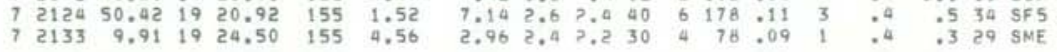
$7 \begin{aligned} & 2141 \quad 47.96 \quad 1920.89 \quad 155 \quad 18.05 \\ & 7\end{aligned}$ 7324
7 $2.88 \quad 19 \quad 24.90 \quad 155 \quad 15.86$

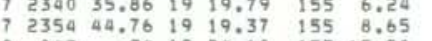

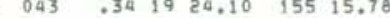

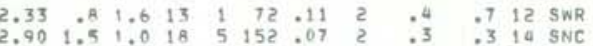

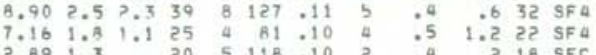

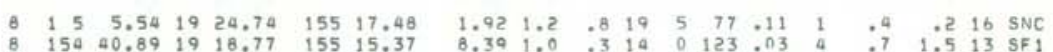

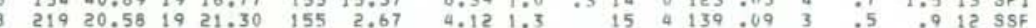

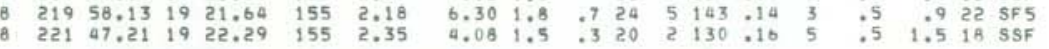

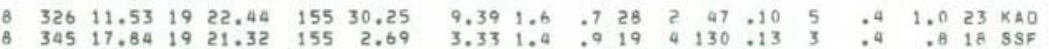

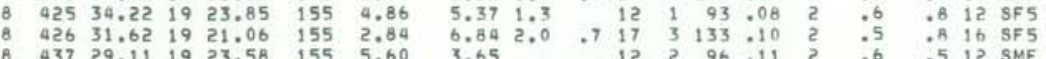

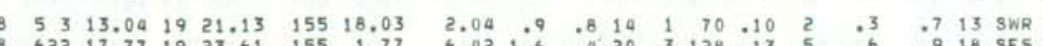

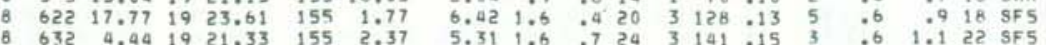


HVO EARTHQUAKE SUMMARY LIST

PAGE 15

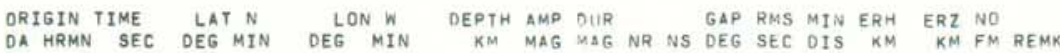

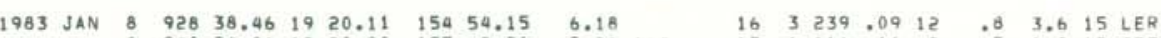

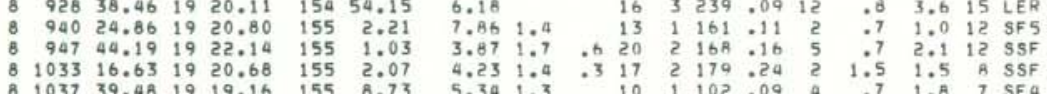

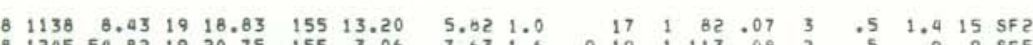

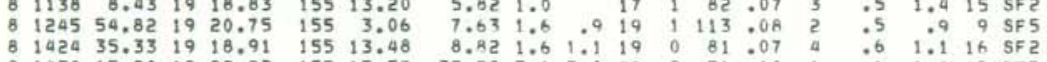

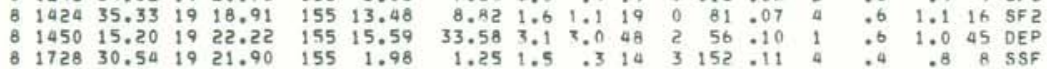

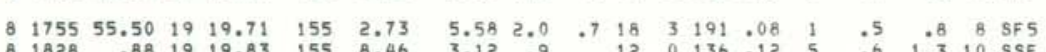

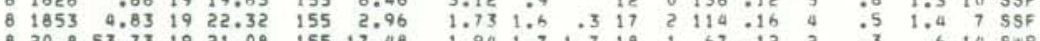

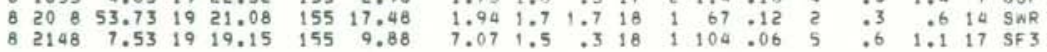

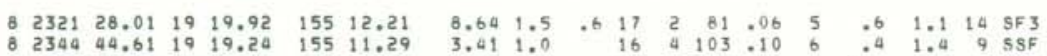

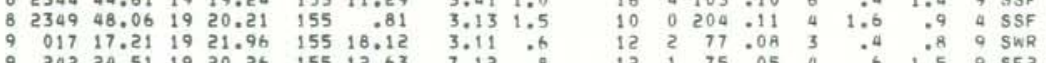

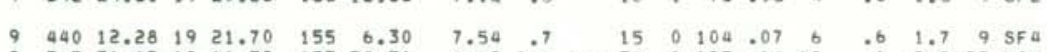

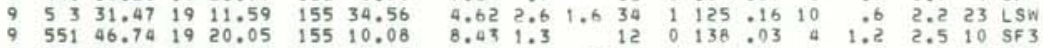

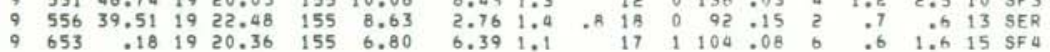
$\begin{array}{lllllllllllllllllllll}659 & 40.54 & 19 & 21.80 & 155 & 1.79 & 4.89 & 2.0 & 1.1 & 26 & 5 & 150 & .13 & 4 & .5 & 1.4 & 14 & \text { SSF } \\ 9 & 7 & 4.73 & 19 & 21.56 & 155 & 1.62 & 5.51 & 2.0 & 1.5 & 24 & 3 & 159 & 1.14 & 4 & .7 & 1.4 & 16 & 5 F 5\end{array}$

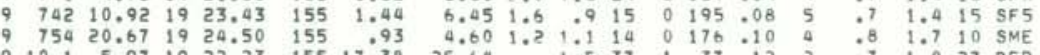

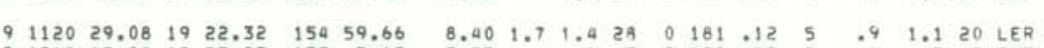

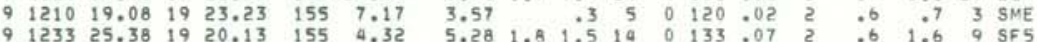

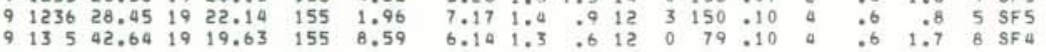

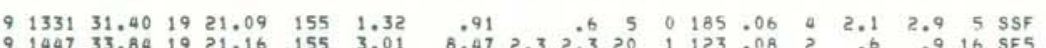

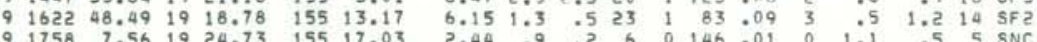

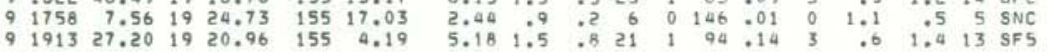
$\begin{array}{lllllllllllllllllllllll}9 & 1916 & 43.83 & 19 & 21.46 & 155 & 16.65 & 25.62 & .9 & 1.1 & 34 & 0 & 63 & .09 & 2 & .7 & 1.0 & 29 & \text { DEP } \\ 9 & 20 & 45.08 & 19 & 22.74 & 155 & 2.65 & 5.99 & 1.4 & & 17 & 1 & 129 & .14 & 4 & 97 & 1.4 & 11 & \text { SF }\end{array}$

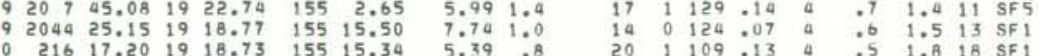

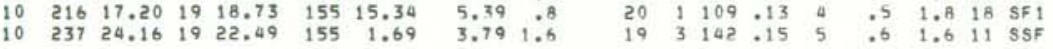

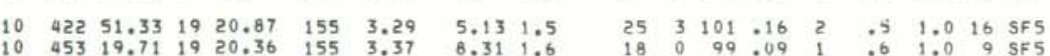

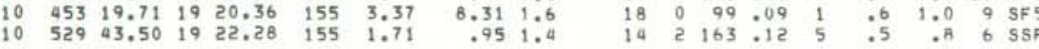

HVO EARTHOUAKE SUMMARY LIST PAGE 16 ORIGIN TIME
DA HRMN SAT N

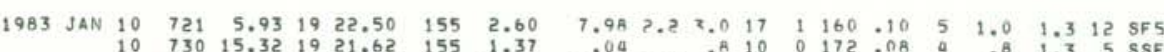

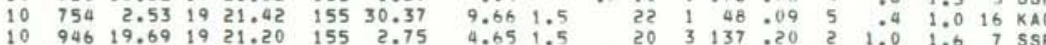

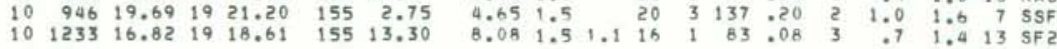

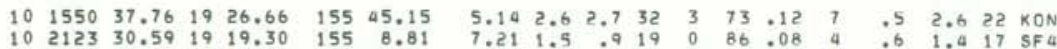

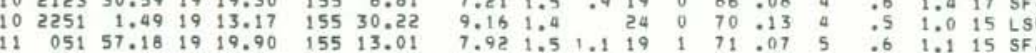

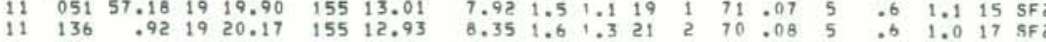

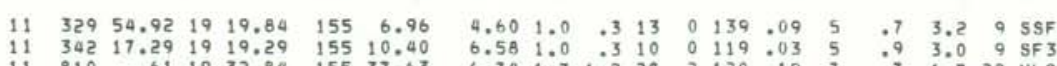

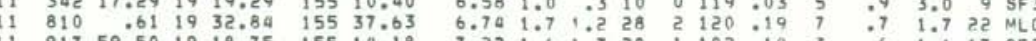

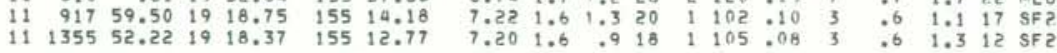

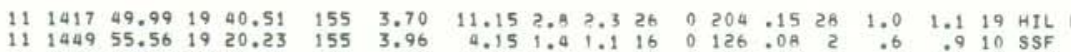

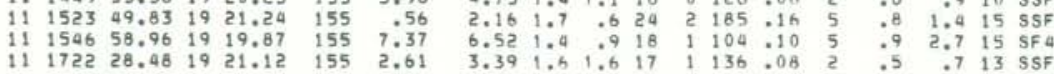

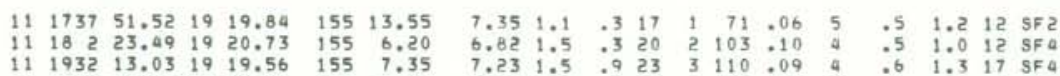

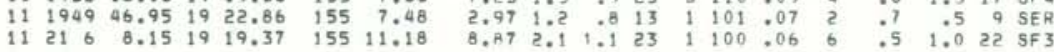

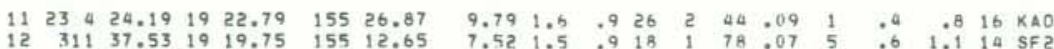

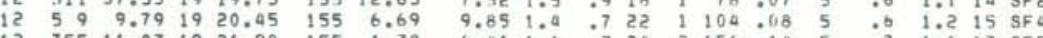

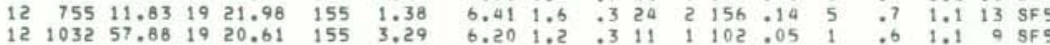

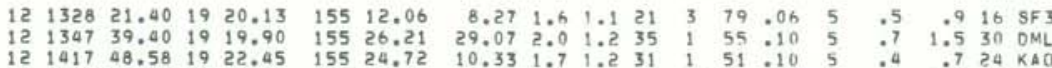

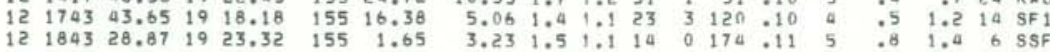

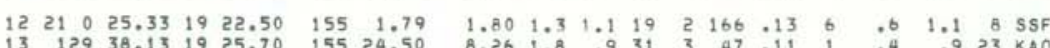

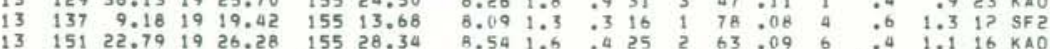

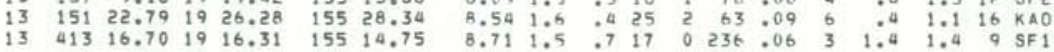

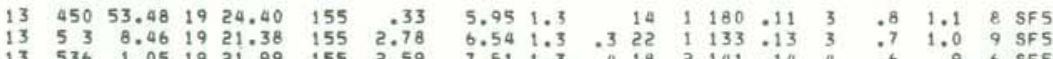

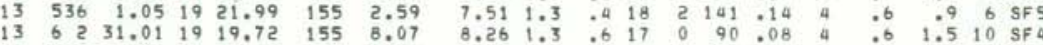

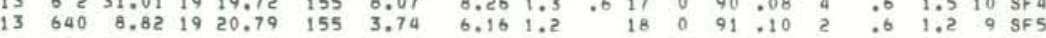
$\begin{array}{lllllllllllllllllll}13 & 838 & 19.09 & 19 & 16.70 & 155 & 15.45 & 3.90 & 1.1 & .6 & 18 & 1 & 210 & .12 & 4 & .8 & 1.1 & 15 & \mathrm{ssF}\end{array}$

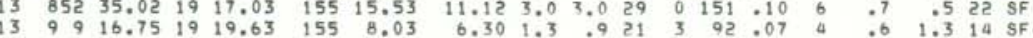


YEAR MON ORIGIN TIME LRMN SEC DEG MIN DEG MIN DEPTH AMP NUR NR GAP RMS MIN ERH ERZ NO

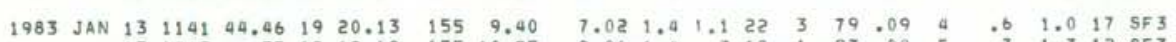

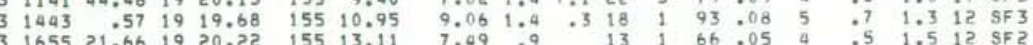

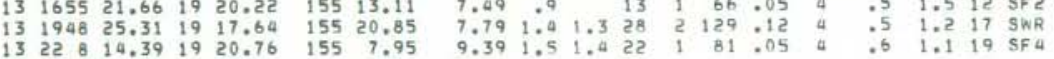

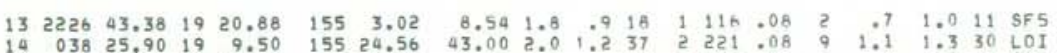
$14 \quad 13559.76 \quad 1920.43 \quad 155 \quad 9.60$

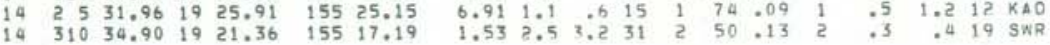

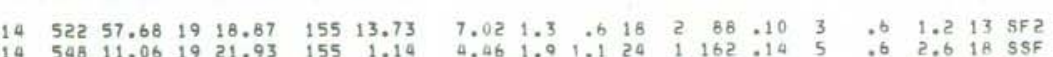

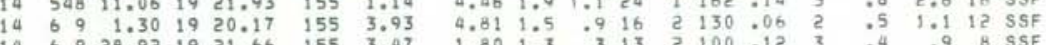

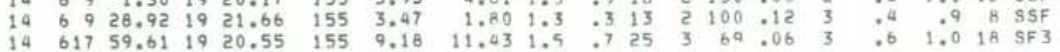

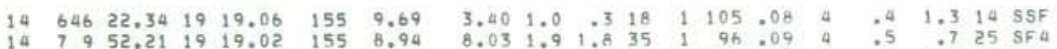

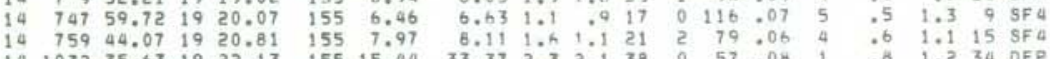

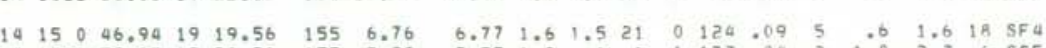

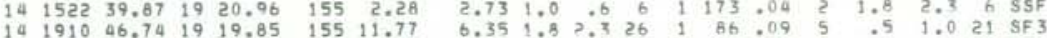

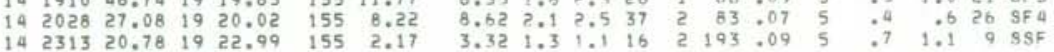

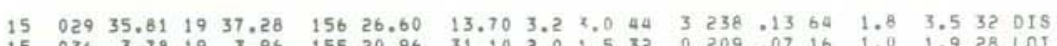

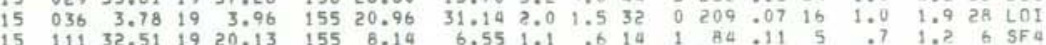

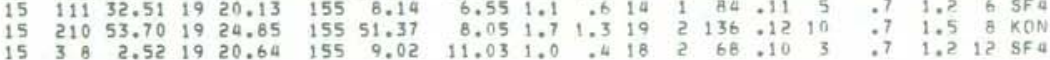

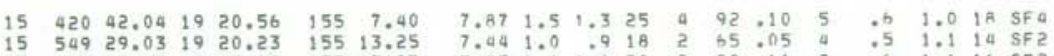

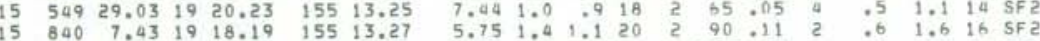

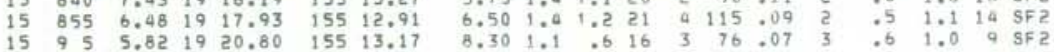

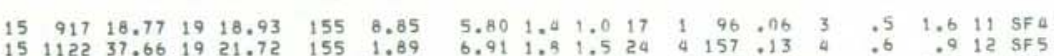

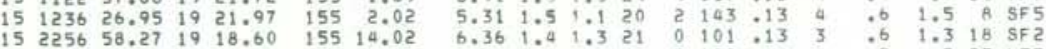

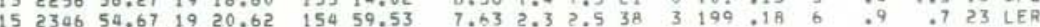

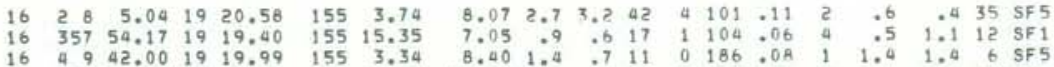

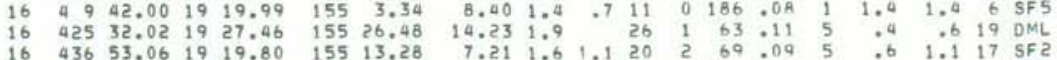

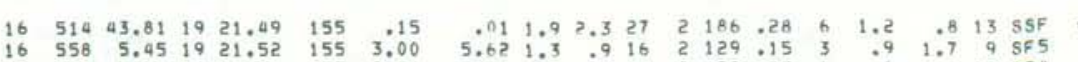

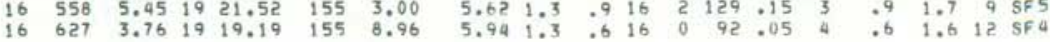
YEAR MON ORIGIN TIME HRMN SEC DEG MIN DEG MIN

1983 JAN $16 \quad 655 \quad 58.74 \quad 19 \quad 19.48 \quad 155 \quad 9.02$

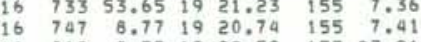
$16 \quad 812 \quad 8.571922 .59 \quad 15523.91$

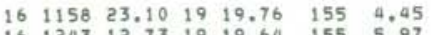

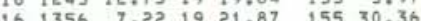

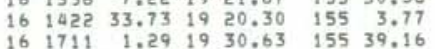
$\begin{array}{lllll}16 & 1826 \quad 39.85 & 19 & 19.42 \quad 155 \quad 14.09\end{array}$ $\begin{array}{lll} & \\ & \end{array}$

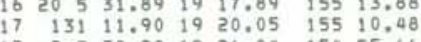

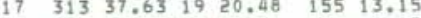
$\begin{array}{lllllll}17 & 351 & 35.32 & 19 & 19.57 & 155 & 8.67\end{array}$

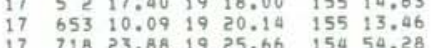

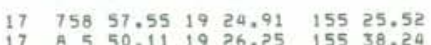

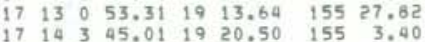
$17714345.0190 .50 \quad 155 \quad 3.40$ $\begin{array}{rrrrrrr}17 & 1728 & 59.67 & 19 & 21.21 & 155 & 4.80 \\ 17 & 1758 & 24.81 & 19 & 16.22 & 155 & 22.82\end{array}$

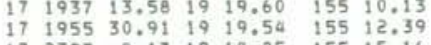
$17 \quad 2325 \quad 50.82 \quad 19 \quad 18.58 \quad 155 \quad 15.12$

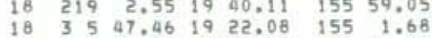
$\begin{array}{llrllll}18 & 58 & 51.06 & 19 & 18.60 & 155 & 13.42 \\ 18 & 934 & 6.52 & 19 & 20.34 & 155 & 11.81\end{array}$ $\begin{array}{lllllll}18 & 948 & 48.31 & 19 & 20.62 \quad 155 & 12.80\end{array}$

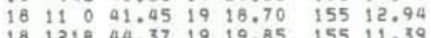

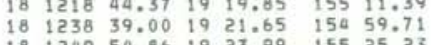
$\begin{array}{llllllll}18 & 15 & 8 & 48.93 & 19 & 16.51 & 155 & 13.10\end{array}$ 16164635.50 19 $22.04 \quad 155,13.89$

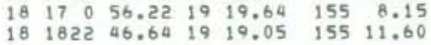
$\begin{array}{lllllll}18 & 2041 & 11.65 & 19 & 20.87 & 155 & 2.31\end{array}$

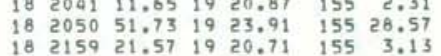

$\begin{array}{lll}\text { DEPTH AMP NUR } & \text { GAP RMS MIN ERH ERZ NO } \\ \text { KM MAG MOG NR NS DEG SEC OIS KM KM FM REMK }\end{array}$

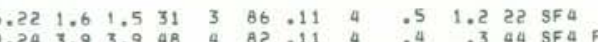

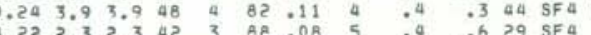

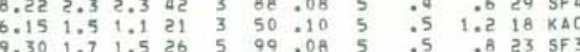

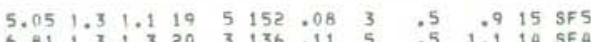

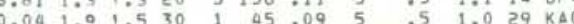

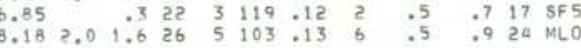
$\begin{array}{lllllllllllll}8.52 & 2.1 & 7.1 & 38 & 1 & 77 & .09 & 5 & .4 & .6 & 34 & \text { SF } 2\end{array}$

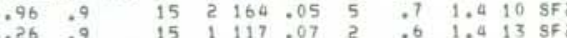

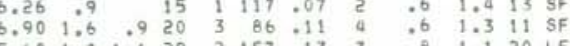
$\begin{array}{llllllllllll}8.01 & 1.2 & .6 & 19 & 1 & 63 & 08 & 4 & .6 & 1.1 & 10 & 5 F 2\end{array}$

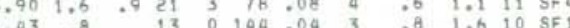
$\begin{array}{lllllllllllll}5.31 & 0 & 17 & 2 & 66 & 13 & 5 & 0 & 5 & 1.8 & 11 & \text { SF } \\ 7.06 & 5 & 1.0 & 17 & 1 & 177 & 13 & 6 & 1.0 & 1.5 & 8 & \text { LEP }\end{array}$

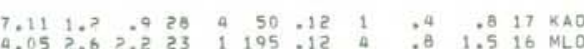

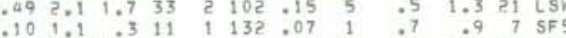

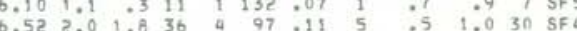

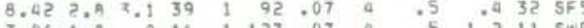

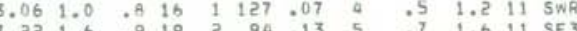

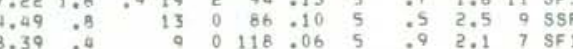
$\begin{array}{llllllllllll}8.48 & 1.0 & .6 & 14 & 2 & 126 & .03 & 4 & .7 & 1.7 & 13 & 5 F\end{array}$

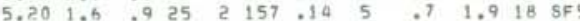

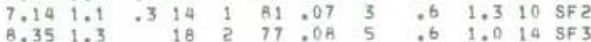

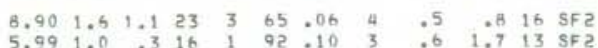

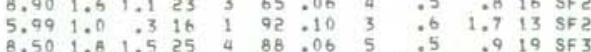

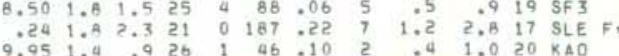

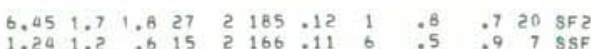

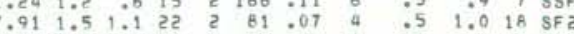

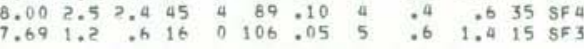
$\begin{array}{llllllllllll}7.11 & 2.4 & 2.3 & 35 & 2 & 155 & .12 & 2 & .5 & .5 & 27 & \mathrm{SF} 5\end{array}$

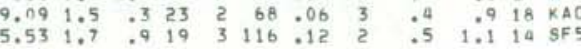


HVO EARTHQUAKE SUMMARY LIST

PAGE 19

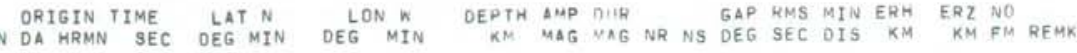

1983 JAN $19 \quad 13348.991922 .22 \quad 155 \quad .85$

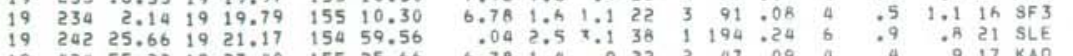

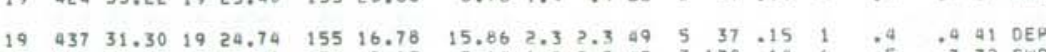

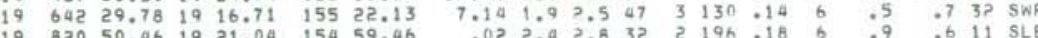

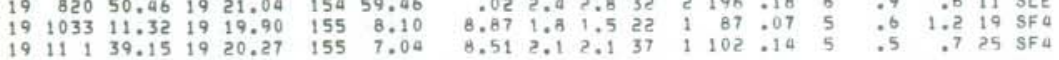
$\begin{array}{lllllll}19 & 1143 & 1.06 & 19 & 21.28 & 155 & 2.43\end{array}$

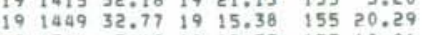
191514
19
19

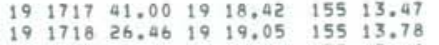

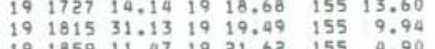

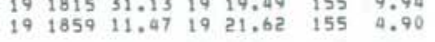
$\begin{array}{lllllll}19 & 19 & 38.42 & 19 & 21.76 & 155 & 5.04\end{array}$ $19223748.091917 .55 \quad 15512.79$

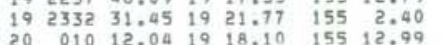

$\begin{array}{llllllll}20 & 215 & 49.27 & 19 & 20.74 & 155 & 12.44\end{array}$

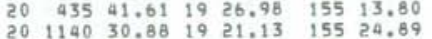

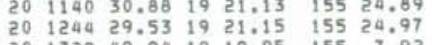
$\begin{array}{lllllll}20 & 1341 & 15.59 & 19 & 19.18 & 155 & 8.35\end{array}$ $20,1629 \quad 32 \quad 19 \quad 19,79 \quad 155,3,73$ $\begin{array}{lllllll}20 & 1727 & 42.93 & 19 & 21.09 & 155 & 12.46 \\ 20 & 1750 & 40.35 & 19 & 20.99 & 155 & 2.63\end{array}$ $20 \quad 2038 \quad 32.81 \quad 1920.94 \quad 155 \quad 12.95$

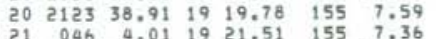
$\begin{array}{lllllll}21 & 046 & 4.01 & 19 & 21.51 & 155 & 7.36 \\ 21 & 122 & 36.12 & 19 & 25.65 & 155 & 37.83\end{array}$ $21 \quad 520 \quad 24.01 \quad 19 \quad 17.33 \quad 155 \quad 32.71$

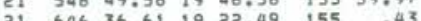

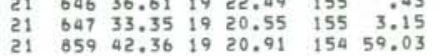
$21 \quad 921 \quad 50.14 \quad 19 \quad 16.46 \quad 155 \quad 21.29$

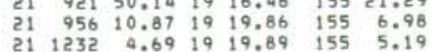

$\begin{array}{llllllllllll}6.94 & 2.4 & 7.5 & 39 & 4 & 141 & .12 & 3 & .5 & .6 & 27 & \text { SF } 5\end{array}$

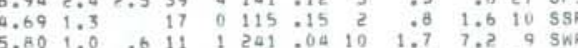

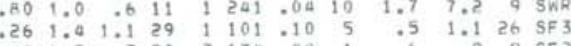

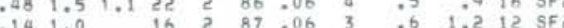

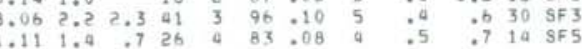
$\begin{array}{llllllllllll}2.84 & 1.3 & 18 & 0 & 118 & .08 & 3 & 1.0 & 1.7 & 13 & \mathrm{SF} 5\end{array}$

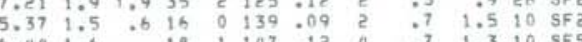

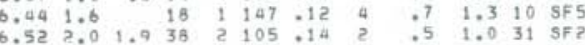

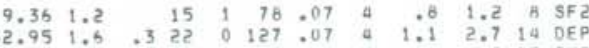

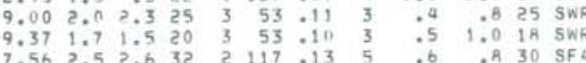
$\begin{array}{llllllllllll}6.33 & 1.3 & 25 & 4 & 87 & .09 & 3 & .5 & .9 & 54 & 5 F 4\end{array}$

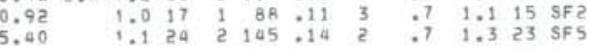

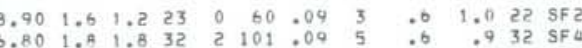

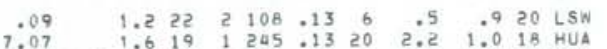

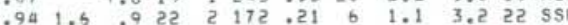

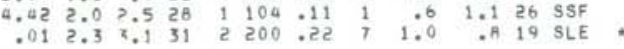

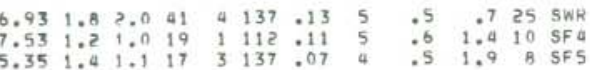

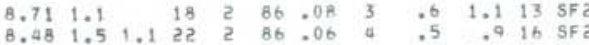

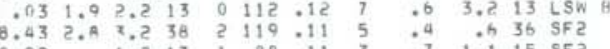

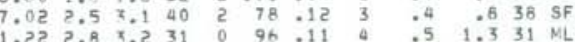

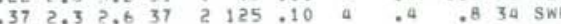

HVO EARTHQUARE SUMMARY LIST

PAGE 20

ORIGIN TIME LAT N LON W OEPTH AMP THR GAP KMS MIN ERH ERZ NO YEAR MON DA MRMN TIMEC DEG MIN DEG MIN DEPTh

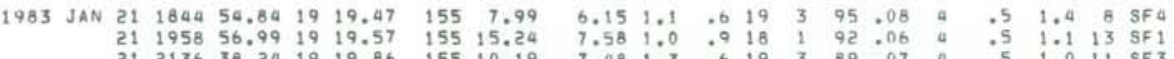

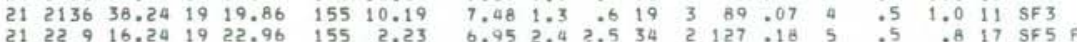

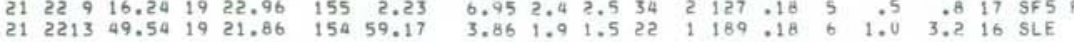

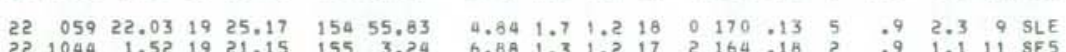

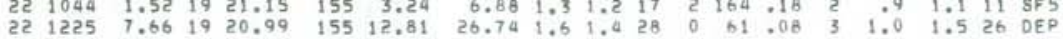

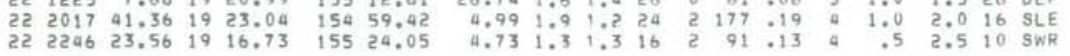

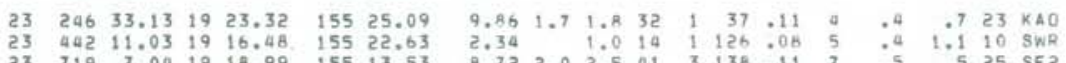

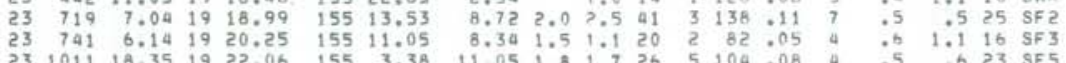

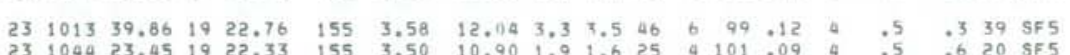

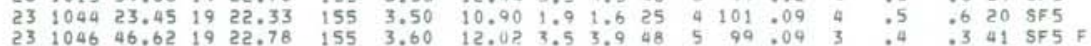

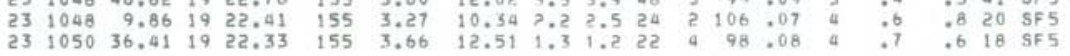

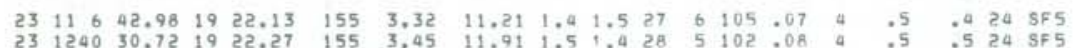

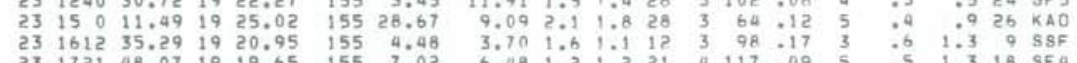

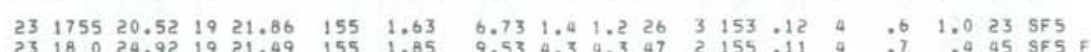

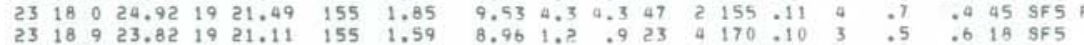

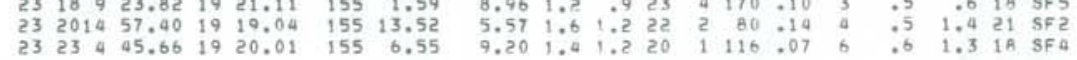

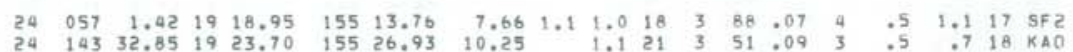

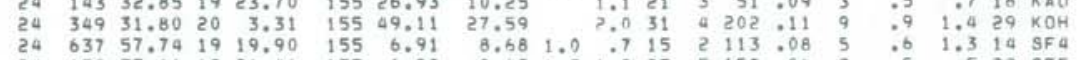

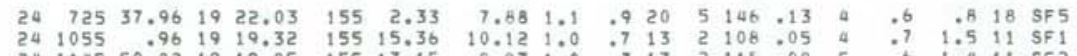

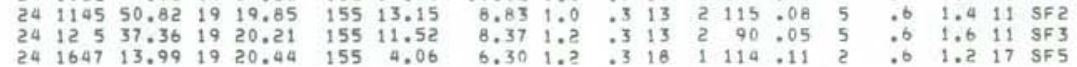

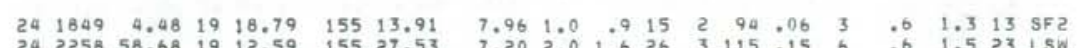

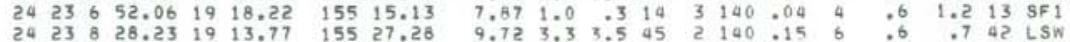

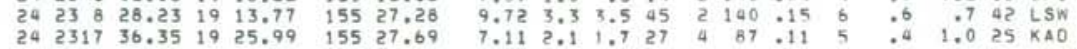

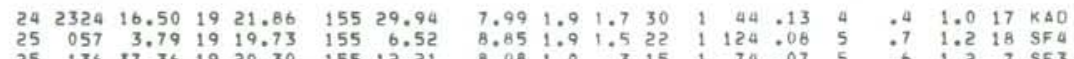


HVO EARTHQUAKE SUMMARY LIST

PAGE 21

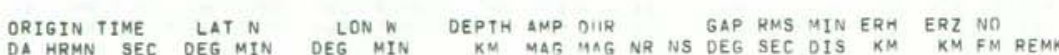

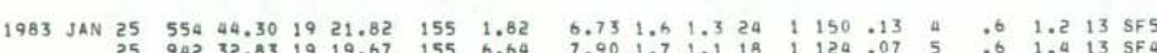

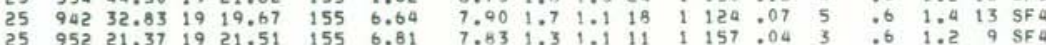

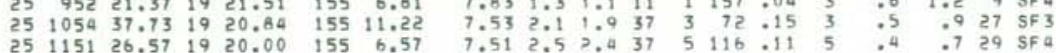

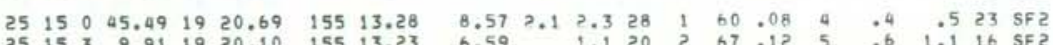

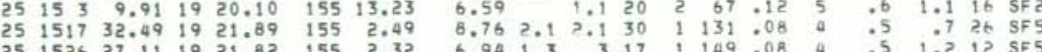

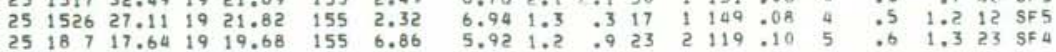

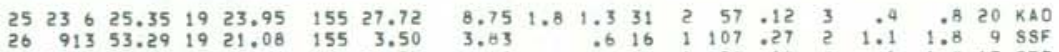

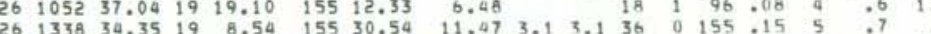

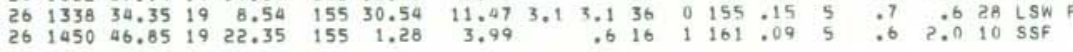

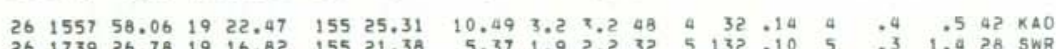

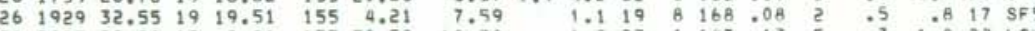

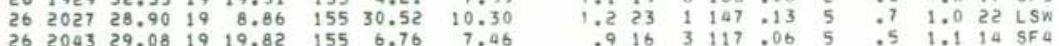

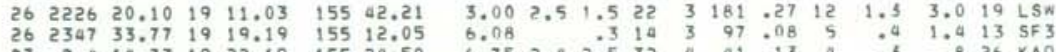

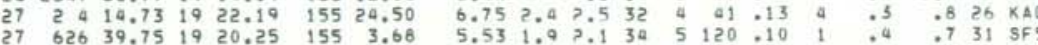

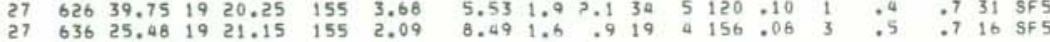

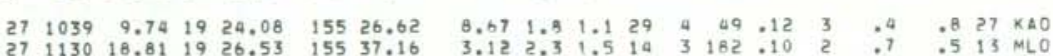

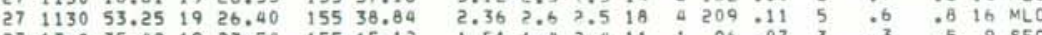

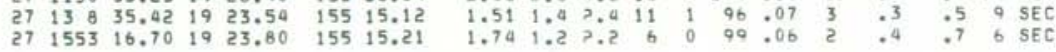

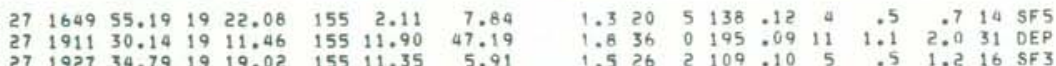

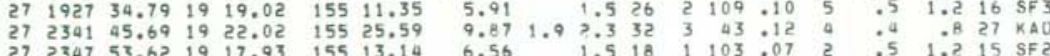

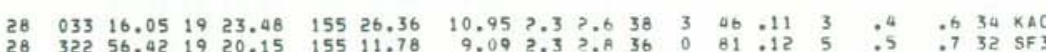
38 (2)

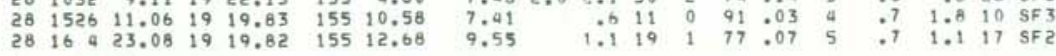

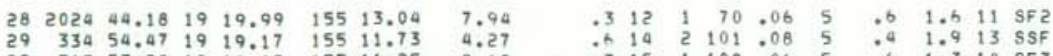

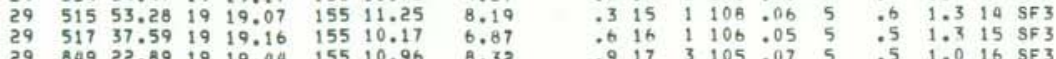

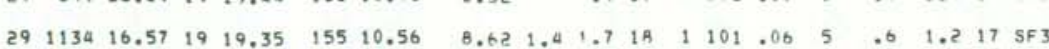
$\begin{array}{lllllllllllllllllll}29 & 1134 & 16.57 & 19 & 19.35 & 155 & 10.56 & 8.62 & 1.4 & 1.7 & 18 & 1 & 101 & .06 & 5 & .6 & 1.2 & 17 & 5 F 3 \\ 29 & 1241 & 5.91 & 19 & 19.01 & 155 & 9.27 & 6.52 & & 3 & 17 & 1 & 101 & 07 & 4 & 07 & 1.5 & 15 & 5 F 3 \\ 29 & 1246 & 17.43 & 19 & 19.09 & 155 & 13.98 & 7.96 & & .3 & 17 & 2 & 90 & .06 & 4 & .5 & 1.2 & 16 & 5 F 2\end{array}$
HVO EARTHQUAKE SUMMARY LIST

PAGE 22 ORIGIN TIME LATN LONW DEPTH AMP MIR GAP RMS MIN ERH ERZ NO $\begin{array}{llllllllllllllllllll}983 & \text { JAN } 29 & 14 & 0 & 20.34 & 19 & 21.99 & 155 & 6.19 & 7.86 & .6 & 15 & 2 & 89 & .07 & 2 & .5 & .8 & 14 & \text { SF } 4\end{array}$

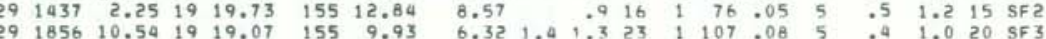

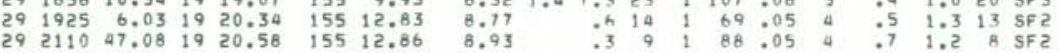

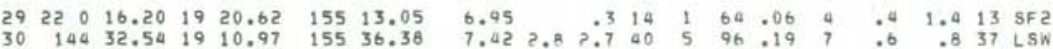

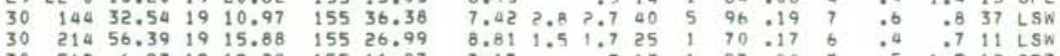

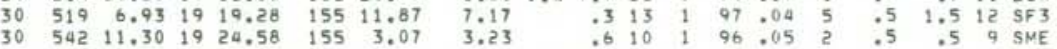

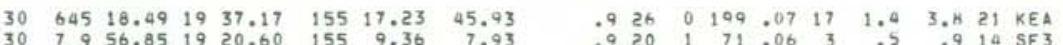

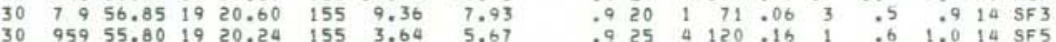

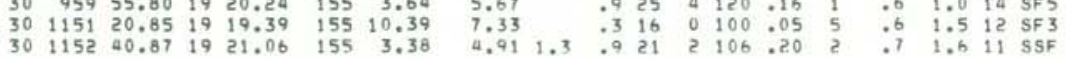

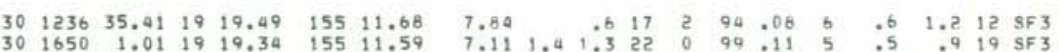

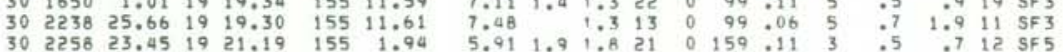

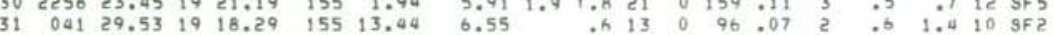

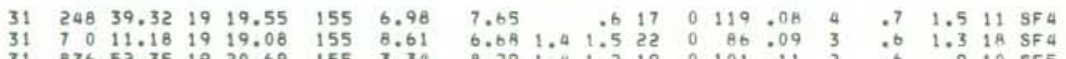

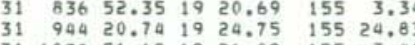

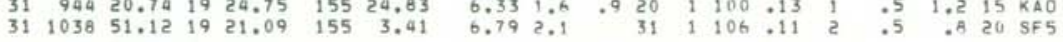

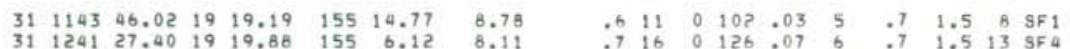

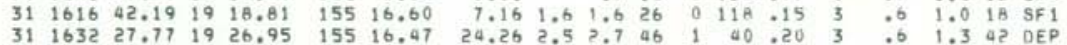

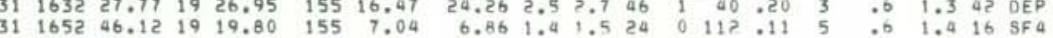

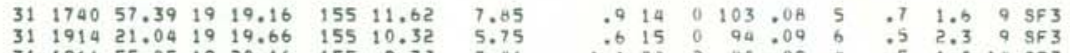

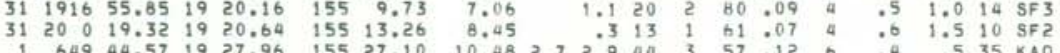

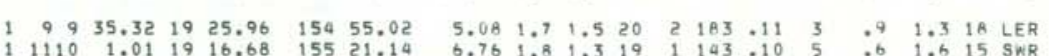

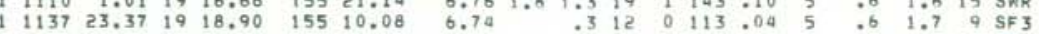

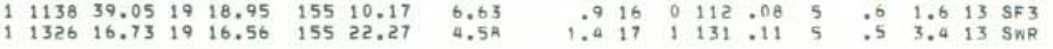

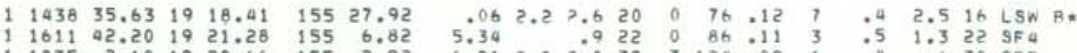

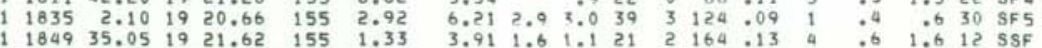

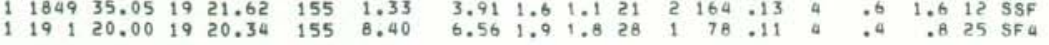

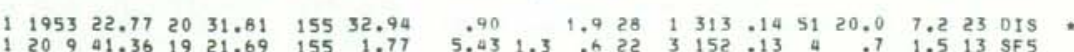

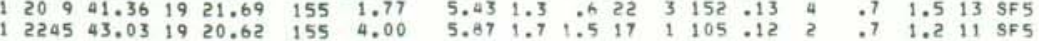




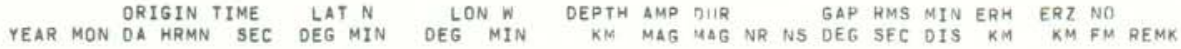

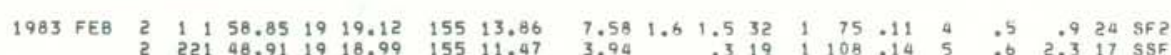

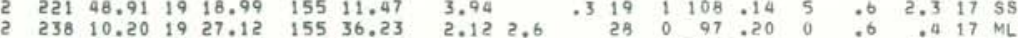

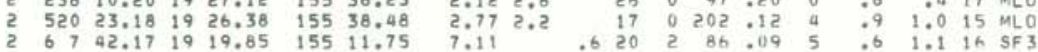

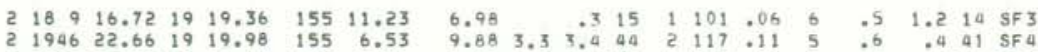

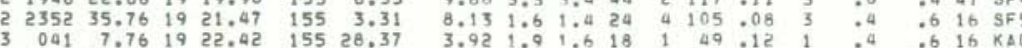
$\begin{array}{lllllllllllllllllll}3 & 041 & 7.76 & 19 & 22.42 & 155 & 28.37 & 3.92 & 1.9 & 1.6 & 18 & 1 & 49 & 12 & 1 & .4 & .6 & 16 & K A 0 \\ 3 & 636 & 47.00 & 19 & 20.73 & 155 & 9.97 & 8.17 & & .3 & 1 A & 1 & 72 & .08 & 2 & .5 & 1.0 & 15 & 5 F 3\end{array}$

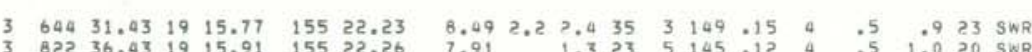

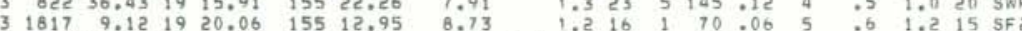

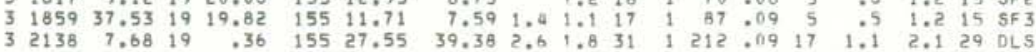

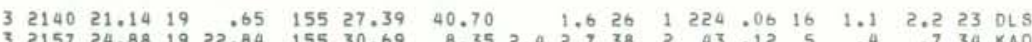

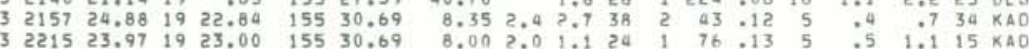

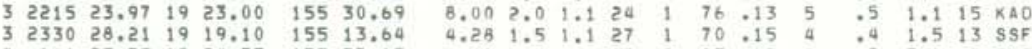

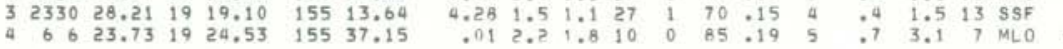

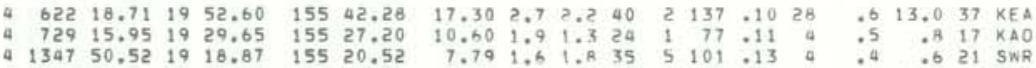
4
4350
4
4624

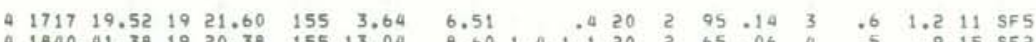
4.645
45224.38
5

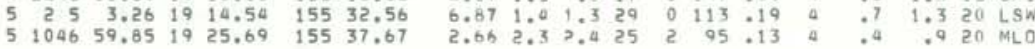

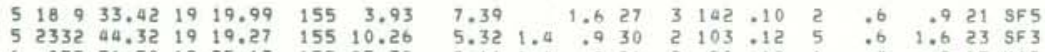

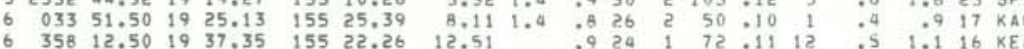

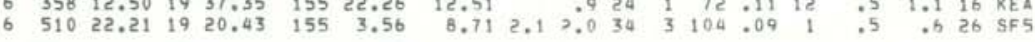

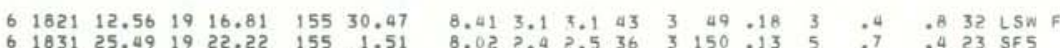

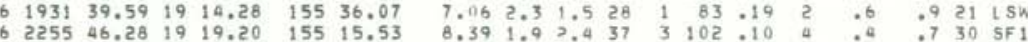

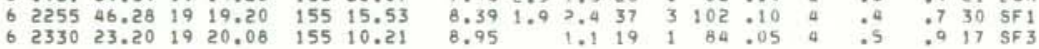

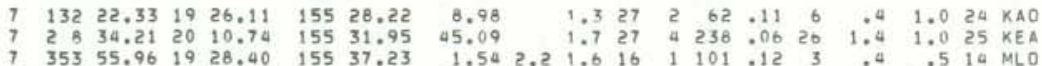

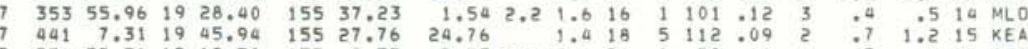

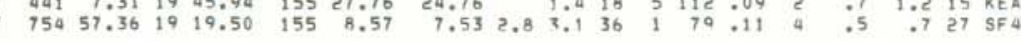

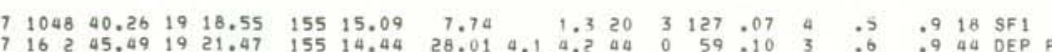

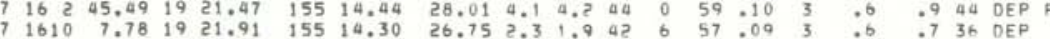

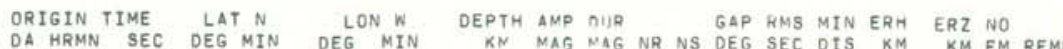
$\begin{array}{lllllllllllllllllllll}983 \text { FEB } & 7 & 1611 & 18.81 & 19 & 20.84 & 155 & 2.55 & 7.87 & 2.3 & 2.3 & 33 & 2 & 144 & .12 & 2 & .6 & .4 & 31 & \text { SFS }\end{array}$ 7174950.701920 .05
755

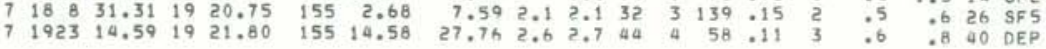

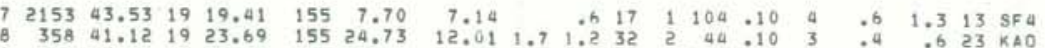

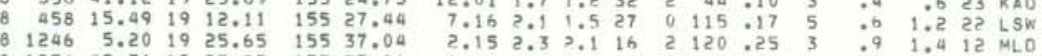

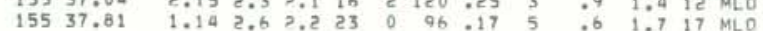

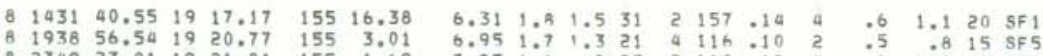

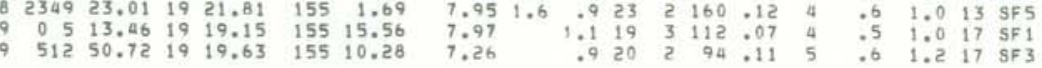
$\begin{array}{llllllllllllllllllll}9 & 553 & 18.20 & 19 & 18.67 & 155 & 13.60 & 5.67 & 1.4 & 1.3 & 24 & 3 & 87 & .11 & 3 & .5 & 1.3 & 18 & 5 F 2\end{array}$

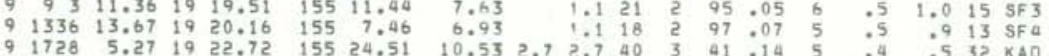

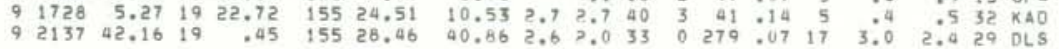

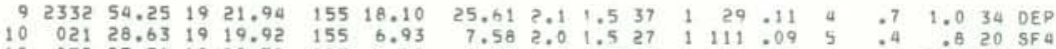

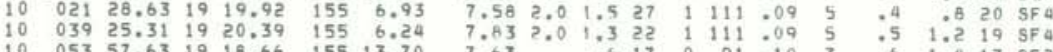

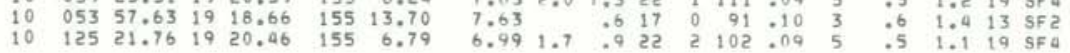
$\begin{array}{llllllllllllllllll}10 & 254 & 32.48 & 19 & 48.04 & 155 & 42.75 & 20.56 & 2.9 & 3.0 & 38 & 0 & 205 & .11 & 32 & 1.8 & 5.0 & 35 \\ 10 & \text { KEA }\end{array}$

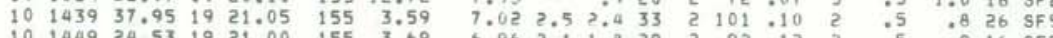

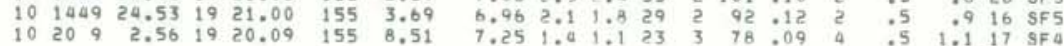

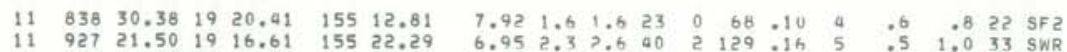

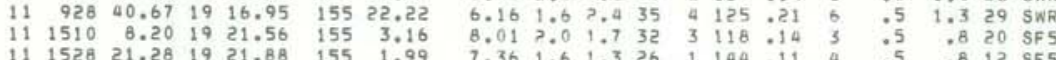

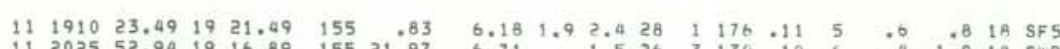

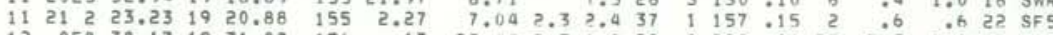

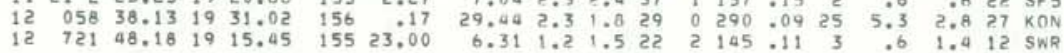

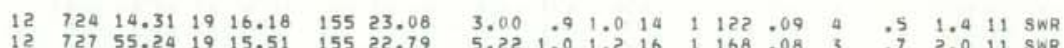

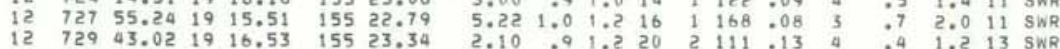

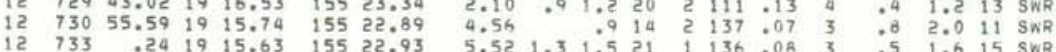

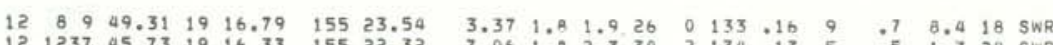

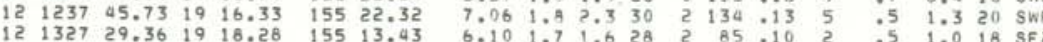


HVO EARTHQUAKE SUMMARY LIST

PAGE 25

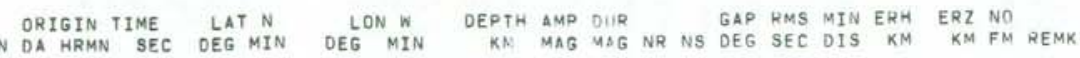

1983 FEB $12 \quad 2328 \quad 16.98 \quad 19 \quad 19.37 \quad 155 \quad 7.09$

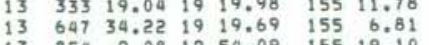

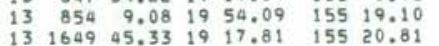

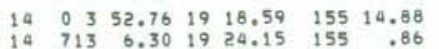
14.1306 .301924 .15 155 $\quad .86$

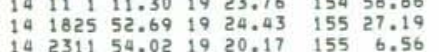

$\begin{array}{llllll}15 & 043 & 19.69 & 19 & 21.22 \quad 155 \quad 2.46\end{array}$ $15 \quad 047 \quad 4.851919 .36 \quad 155 \quad 11.24$

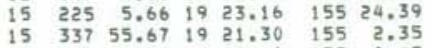
$15125940.2019 \quad 20.60 \quad 155 \quad 12.99$ $1519620.421919 .65 \quad 155 \quad 7.53$ 15
16 $70 \begin{aligned} & 51.23 \\ & 16\end{aligned}$ $\begin{array}{lllllll}16 \quad 1339 & 8.08 \quad 19 & 22.22 & 155 & 5.11\end{array}$ $\begin{array}{llllllllllllll}.79 & 1.9 & 1.3 & 23 & 3 & 121 & -11 & 4 & .4 & 1.3 & 11 & \text { SSF } \\ .90 & .5 & .3 & 23 & 2 & 83 & .07 & 5 & .5 & 1.0 & 19 & \text { SF3 }\end{array}$

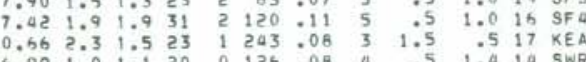

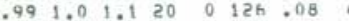
$\begin{array}{lllllllllllll}7.06 & 1.2 & 1.1 & 28 & 2 & 105 & .09 & 4 & .5 & .8 & 14 & \mathrm{SF} \\ 5.96 & & 1.1 & 20 & 2 & 170 & .12 & 4 & .0 & 1.2 & 10 & \mathrm{SF} 5\end{array}$

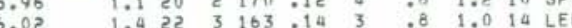
$\begin{array}{llllllllllllll}6.01 & 1.8 & .3 & 27 & 2 & 60 & 11 & 4 & .5 & .9 & 14 & K A 0 \\ 8.86 & 3.4 & 7.4 & 43 & 4 & 112 & .09 & 5 & .0 & .5 & 36 & 3 F 4\end{array}$ $\begin{array}{llllllllllll}.84 & 1.3 & 1.2 & 20 & 2 & 141 & .10 & 3 & .6 & 1.0 & 10 & \mathrm{SF}\end{array}$

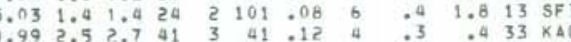

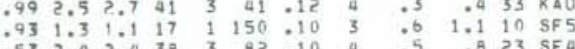
$\begin{array}{llllllllllll}7.37 & 1.2 & 1.3 & 27 & 2 & 64 & .11 & 4 & .5 & .8 & 20 & \text { SF } 2\end{array}$

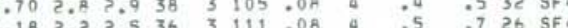

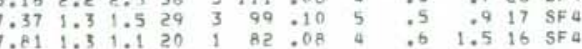

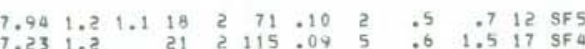

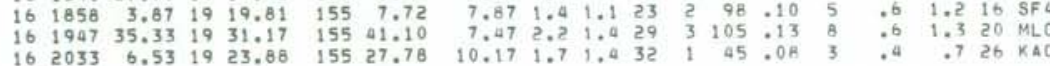

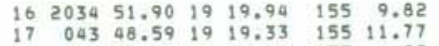

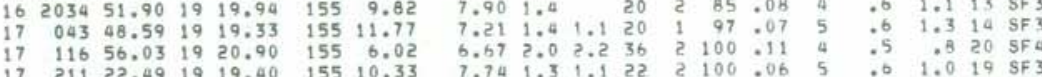

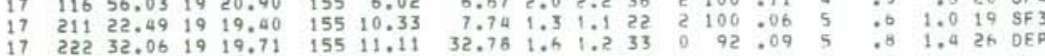

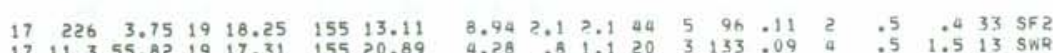

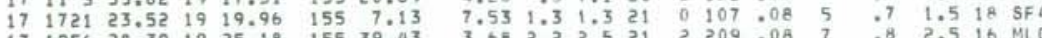

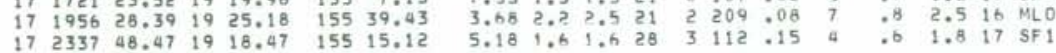
$\begin{array}{lllllll}18 & 818 & 58.54 & 19 & 22.26 & 155 & 4.99\end{array}$

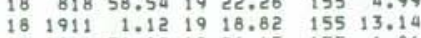

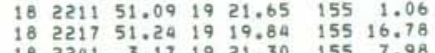
$\begin{array}{llllllll}19 & 0 & 2 & 53.61 & 19 & 14.65 & 155 & 32.88 \\ 19 & 121 & 53.75 & 19 & 26.02 & 155 & 28 & 0.49\end{array}$ $19 \quad 63843.34$ 19 $20.68 \quad 15513.02$

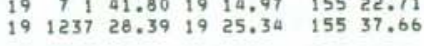

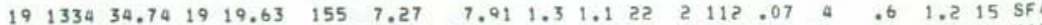

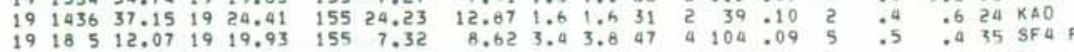

HVO EARTHQUAKE SUMMARY LIST

PAGE 26

ORIGIN TIME LAT N LON W DEPTH AMP RUR GAP RMS MIN ERH ERZ NO

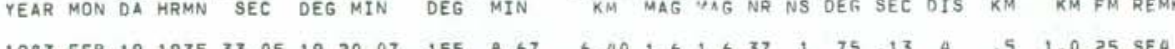

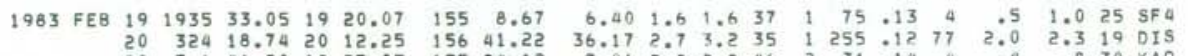

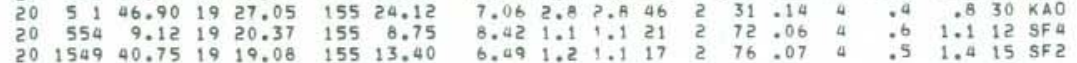

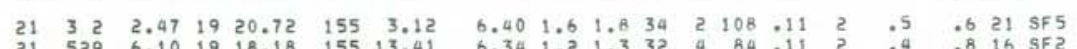

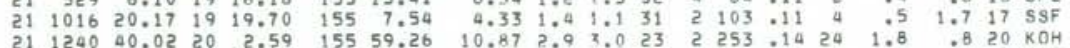

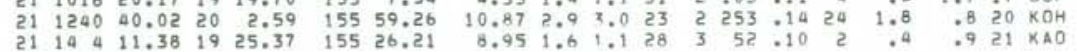

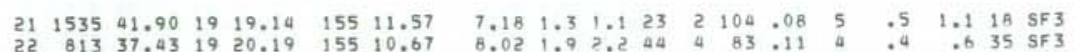

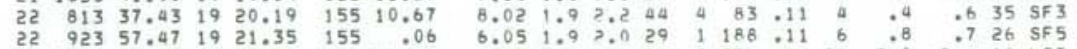

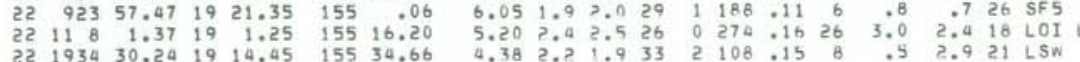

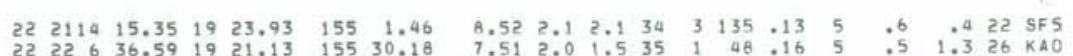

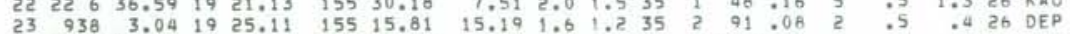

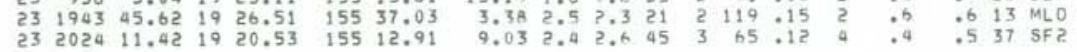

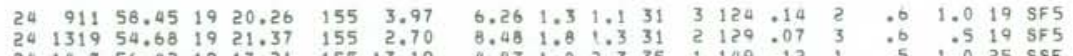

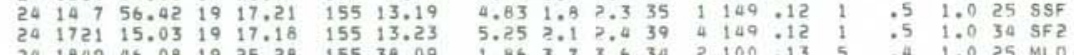
$\begin{array}{llllllllllllllllllll}24 & 22 & 3 & 21.00 & 19 & 19.40 & 155 & 12.31 & 4.69 & 1.5 & 1.3 & 28 & 2 & 89 & .13 & 5 & .4 & 1.8 & 16 & \text { SSF }\end{array}$

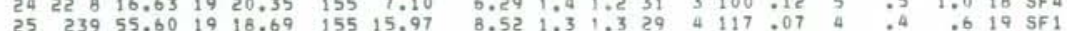

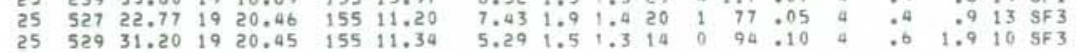

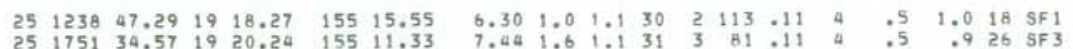

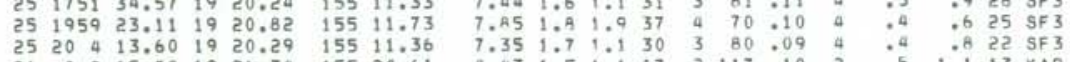
$\begin{array}{lllllll}4.43 & 1.5 & 1.1 & 17 & 2\end{array}$

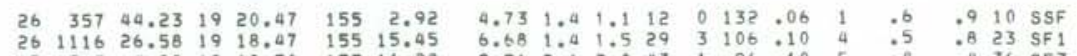

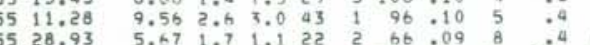

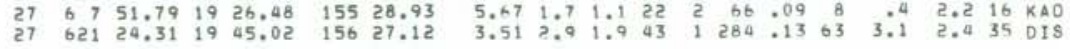

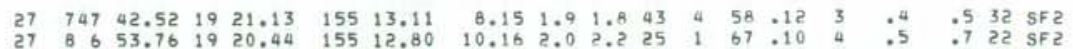

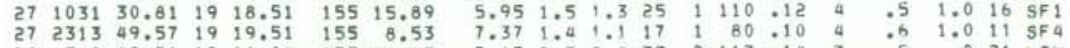

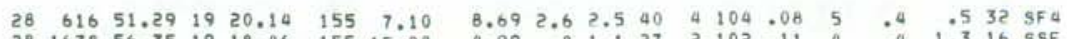

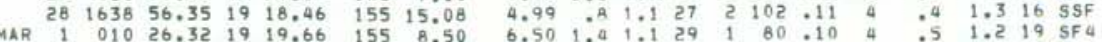


HVO EARTHQUAKE SUMMARY LIST

PAGE 27

ORIGIN TIME
OA HRMN SAT

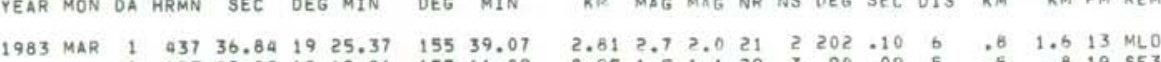

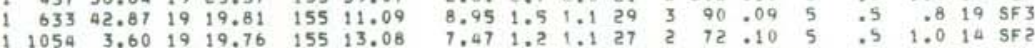

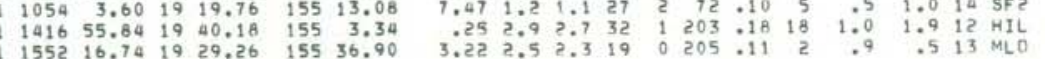

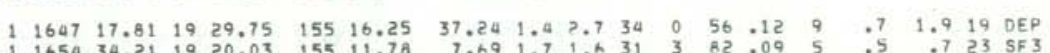

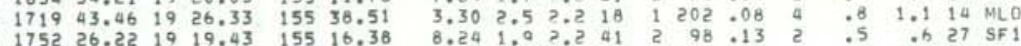

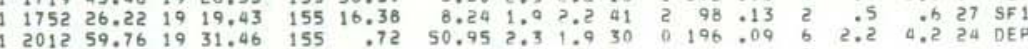
$\begin{array}{lllllllllllllllllll}2 & 220 & 11.01 & 19 & 26.24 & 155 & 35.66 & .26 & 2.4 & 2.5 & 16 & 2 & 97 & .09 & 1 & .2 & .2 & \text { A } & \text { MLO } \\ 2 & 220 & 51.18 & 19 & 19.99 & 155 & 8.40 & 4.96 & 1.1 & 1.1 & 22 & 2 & 81 & : 12 & 5 & .6 & 1.6 & 12 & \text { SSF }\end{array}$

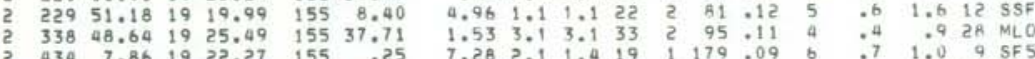

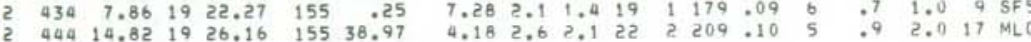
$\begin{array}{llllllllllllllllll}2 & 1359 & 31.39 & 19 & 43.78 & 156 & 5.23 & 25.88 & 2.9 & 2.2 & 24 & 2 & 240 & 11 & 26 & 1.0 & 2.1 & 22 \\ \text { HUA }\end{array}$

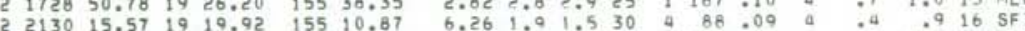

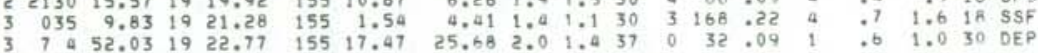

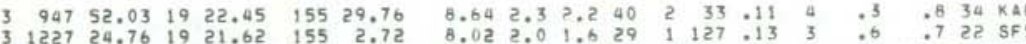

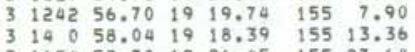

$\begin{array}{lllllll}3 & 1713 & 59.29 & 19 & 20.47 & 155 & 6.76\end{array}$

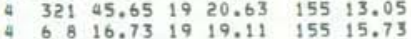

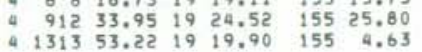

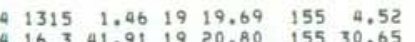
$4 \begin{array}{ll}1615 & 13.951920 .77 \quad 155 \quad 30.45\end{array}$

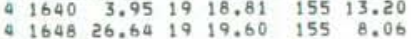
$\begin{array}{lllllll}4 & 1650 & 25.12 & 19 & 16.48 & 155 & 15.11 \\ 4 & 178 & 41.93 & 19 & 21.27 & 155 & 2.46\end{array}$ $4222010.301924 .01 \quad 155 \quad 24.99$

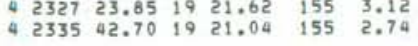
$\begin{array}{llllllll}5 & 011 & 1.21 & 19 & 32.20 & 155 & 42.66 \\ 5 & 217 & 55.79 & 18 & 55.32 & 155 & 29.72\end{array}$

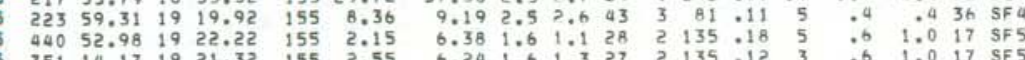

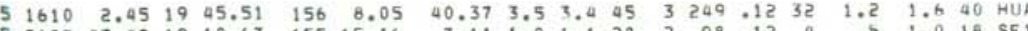

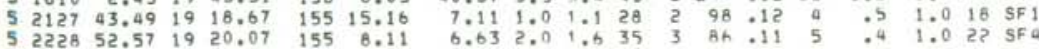

HVO EARTHQUAKE SUMMARY LIST

PAGE 28 ORIGIN TIME LAT N LON W DEPTH AMP OUR N GAP RMS MIN ERH ERZ NO
DA HRMN SEC DEG MIN DEG MIN DEM KM

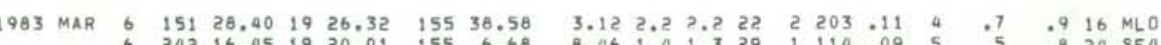

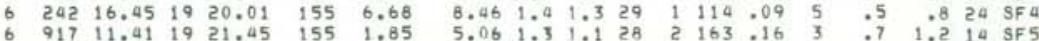

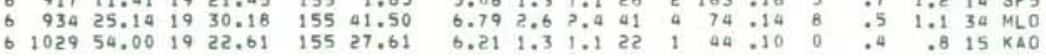

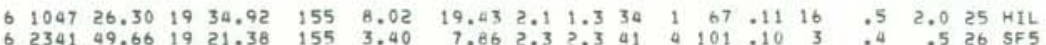

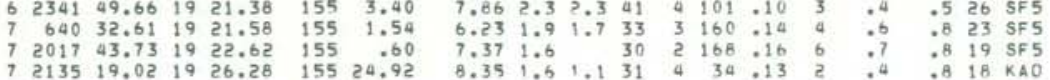

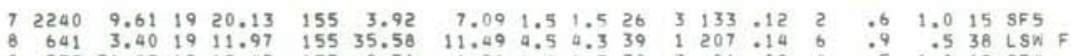

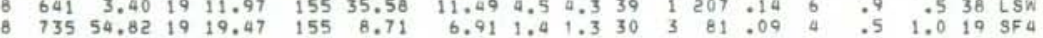

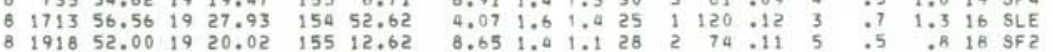

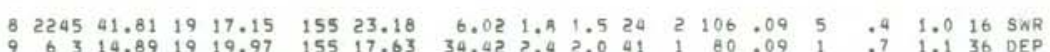

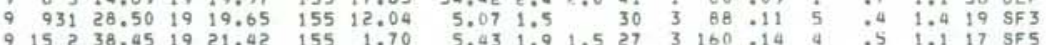

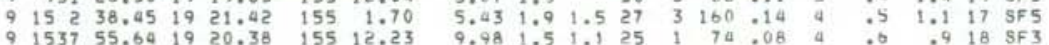

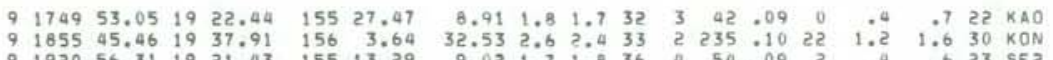

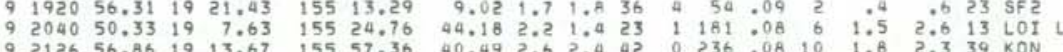
$\begin{array}{lllllllllllllllll}9.22 & 130.54 & 19 & 16.23 & 155 & 23.54 & 3.81 & 1.3 & 20 & 3 & 109 & .11 & 4 & .5 & 1.4 & 17 & \text { SWR }\end{array}$

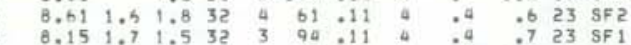

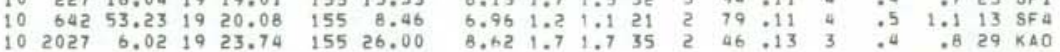

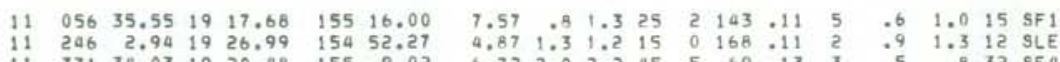

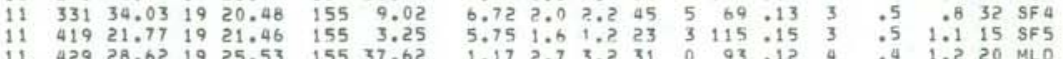

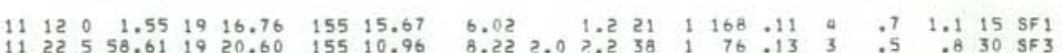

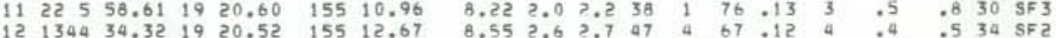

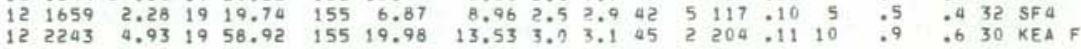

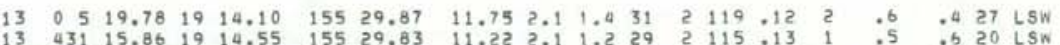

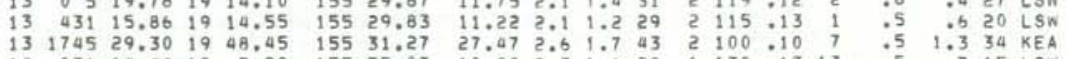

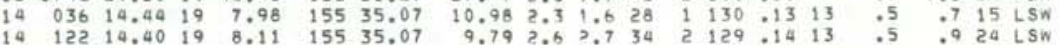

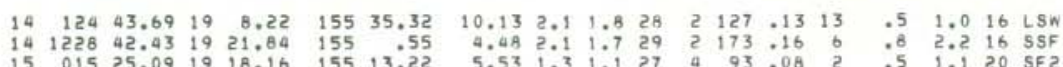




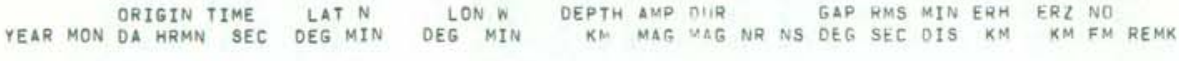

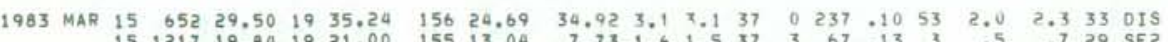

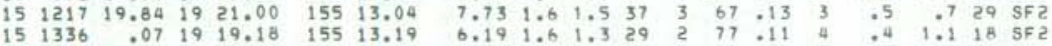

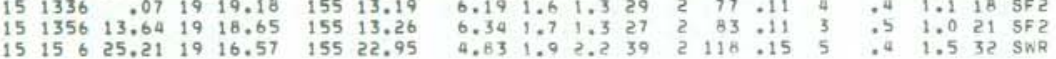

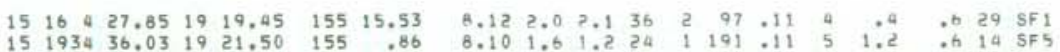

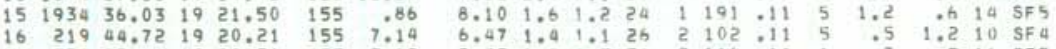

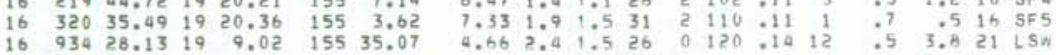

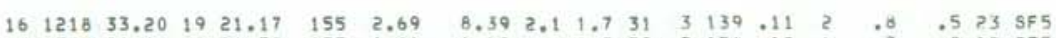

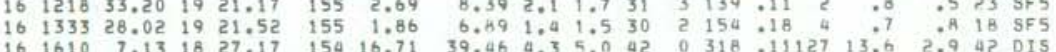

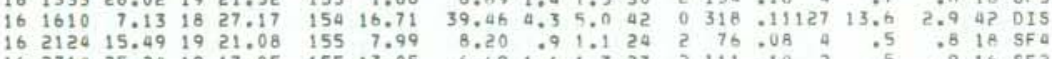

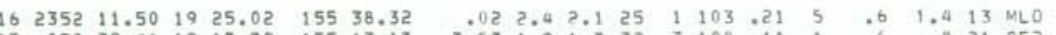

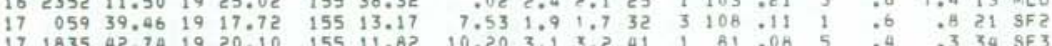

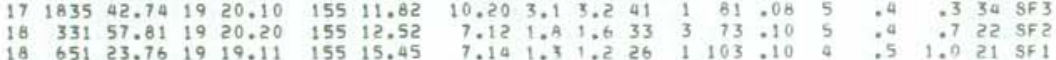

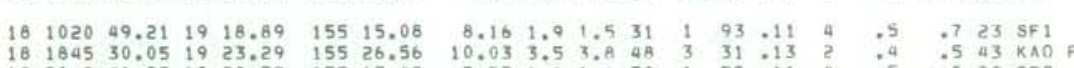

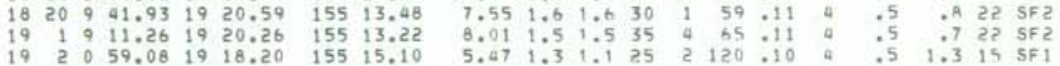

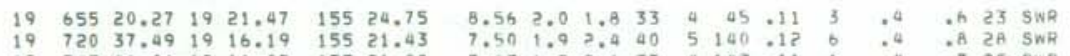

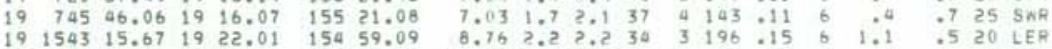

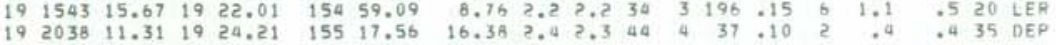

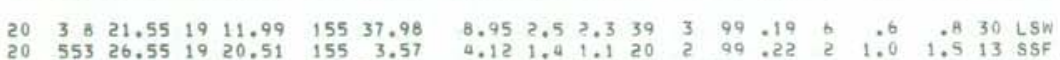

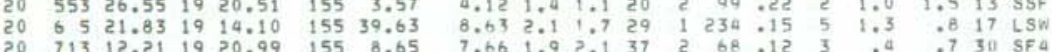

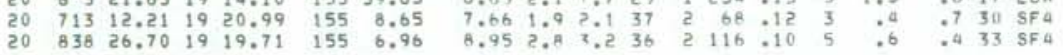

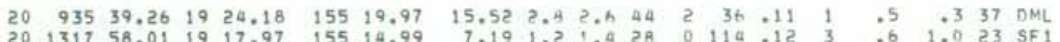

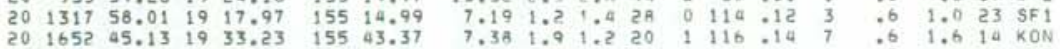

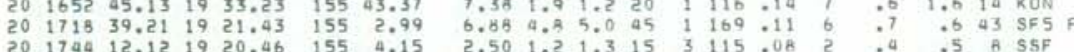
$\begin{array}{llllllllllllllllllll}20 & 1753 & 23.48 & 19 & 20.60 & 155 & 3.33 & 5.55 & 1.4 & 1.5 & 26 & 2 & 99 & .12 & 1 & .6 & .9 & 17 & \text { SF } 5\end{array}$

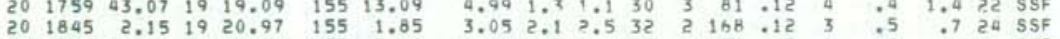

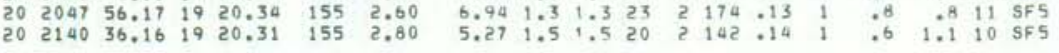

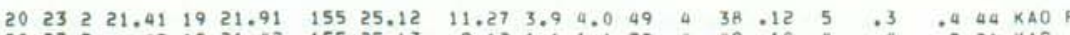

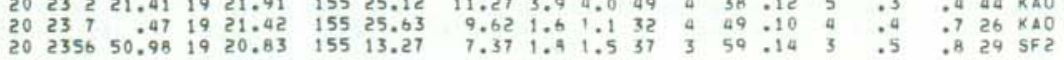
YEAR MON DA HRMN SEC DEG MIN DEG MIN DEM MAG MAG NR NS DEG SEC DIS KM ERM FM REMK

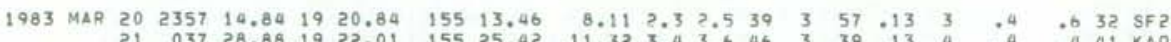

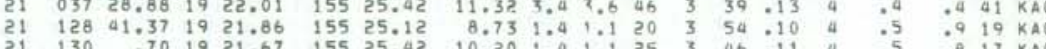

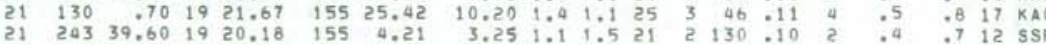

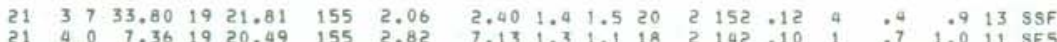

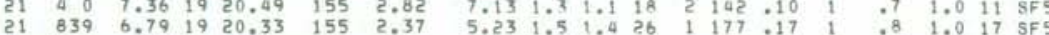

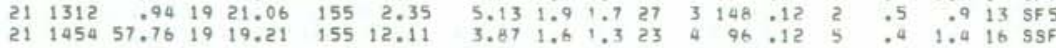

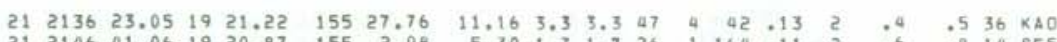

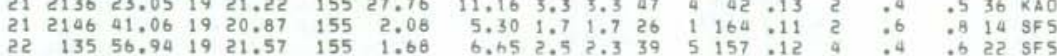

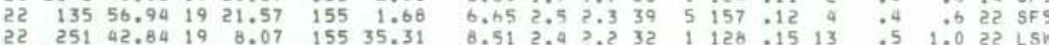

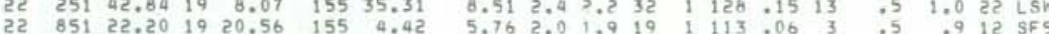

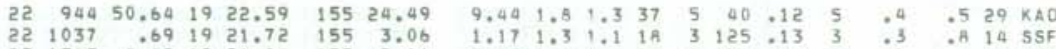

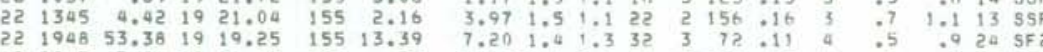

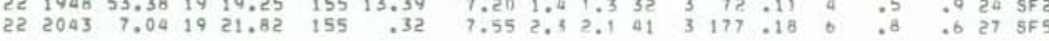

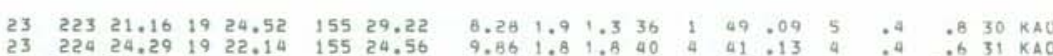

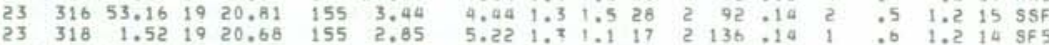

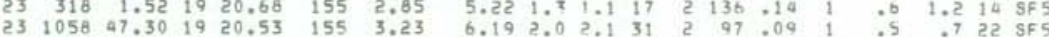

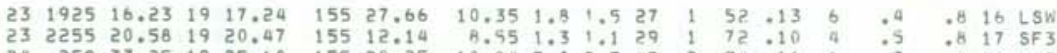

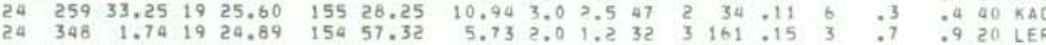

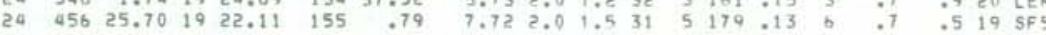

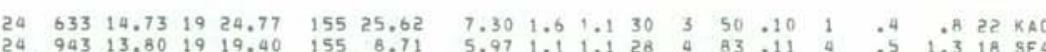

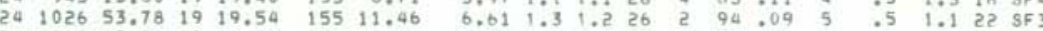

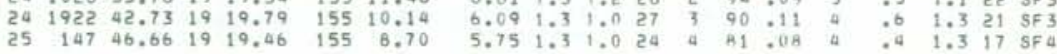
$\begin{array}{llllllllllllllllllll}25 & 2 & 0 & 21.53 & 19 & 23.86 & 155 & 25.88 & 8.73 & 2.3 & 2.7 & 46 & 4 & 39 & .13 & 3 & .4 & .6 & 35 & \mathrm{KAO} \\ 25 & 441 & 23.04 & 19 & 20.24 & 155 & 13.53 & 6.63 & .8 & 1.0 & 20 & 1 & 67 & 0.08 & 5 & .5 & 1.2 & 17 & \mathrm{SF} 2\end{array}$

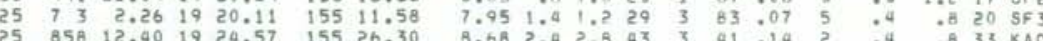

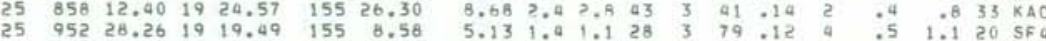

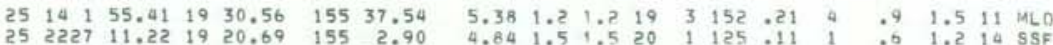

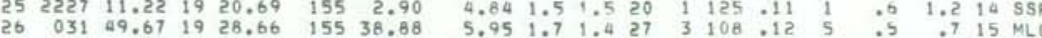

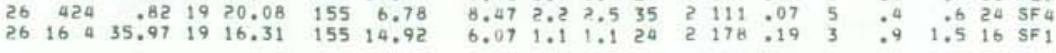

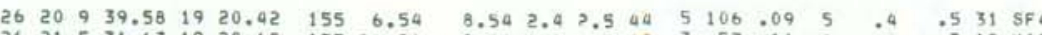

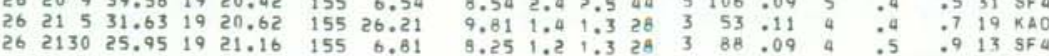


HVO EARTHQUAKE SUMMARY LIST

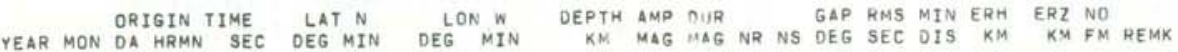
$\begin{array}{llllllllllllllllllll}1983 \text { MAR } & 26 & 2135 & 3.68 & 19 & 19.78 & 155 & 13.63 & 7.63 & 1.6 & 1.3 & 37 & 4 & 65 & .11 & 5 & .4 & .7 & 28 & \text { SF } 2 \\ 26 & 2146 & 42.89 & 19 & 19.56 & 155 & 13.57 & 7.39 & 1.3 & 1.0 & 30 & 2 & 65 & 08 & 5 & .4 & .8 & 18 & \text { SF } 2\end{array}$ $27 \quad 03357.73 \quad 1923.69 \quad 155 \quad 29.57$

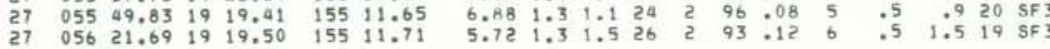

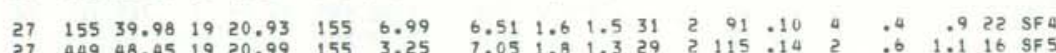

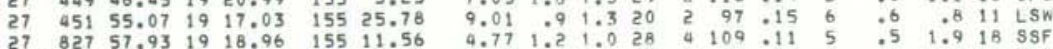

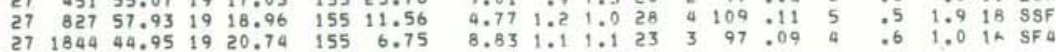
$\begin{array}{llllllllllllllllllll}29 & 031 & 12.45 & 19 & 12.46 & 155 & 29.92 & 7.11 & 2.2 & 1.1 & 31 & 1 & 70 & .21 & 5 & .6 & 1.2 & 16 & \text { LSW } \\ 29 & 257 & 23.02 & 19 & 20.69 & 155 & 2.44 & 5.93 & 1.3 & .9 & 19 & 3 & 155 & .10 & 2 & .5 & .9 & 10 & \text { SF }\end{array}$

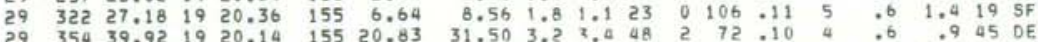

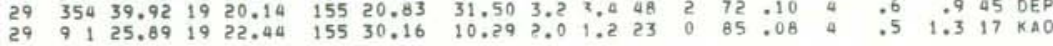

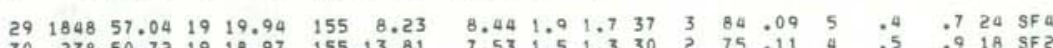

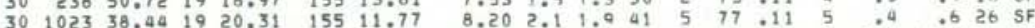

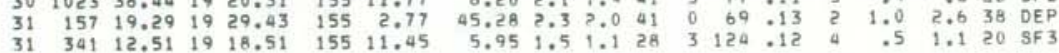

$533 \quad 49.41923 .34 \quad 15526.52$

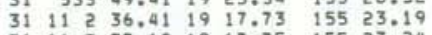

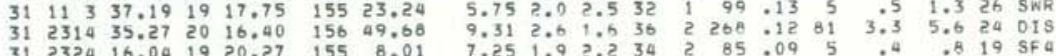

APR 1 2 $2259.68 \quad 1945.33 \quad 155 \quad 50.23$

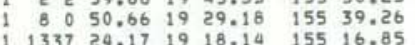

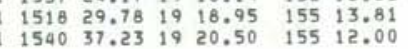

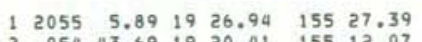

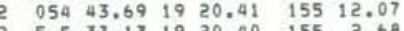

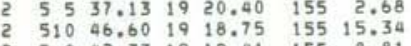
$\begin{array}{lllllll}2 & 1322 & 26.81 & 19 & 30.22 & 155 & 15.14 \\ 2 & 1519 & 58.62 & 19 & 11.49 & 155 & 37.83\end{array}$ 2.

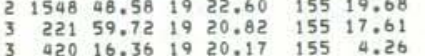
$\begin{array}{lllllll}3 & 1356 & 13.97 & 19 & 20.48 & 155 & 2.47\end{array}$

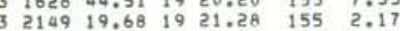
$\begin{array}{lrlllll}4 & 243 & 44.14 & 19 & 19.90 & 155 & 7.62 \\ 4 & 1144 & 47.60 & 19 & 25.82 & 155 & 37.59\end{array}$ $\begin{array}{lllllll}4 & 1232 & 98.04 & 19 & 19.17 & 155 & 13.09 \\ 4 & 1340 & 58.56 & 19 & 24.64 & 154 & 57.30 \\ 4 & 1525 & 53.09 & 19 & 17.71 & 155 & 20.34\end{array}$ $\begin{array}{llllllllllllll}5.36 & 1.9 & 1.7 & 26 & 2 & 187 & .21 & 8 & 2.1 & 1.3 & 17 & \text { HUA }\end{array}$

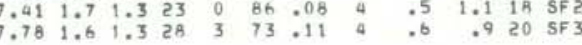
$\begin{array}{lllllllllllll}0.06 & 2.1 & 1.3 & 33 & 2 & 53 & .11 & 8 & .4 & 1.1 & 20 & \text { KAC } \\ 0.72 & .5 & -5 & 30 & 1 & 74 & 09 & 4 & .5 & .7 & 23 & \text { SF }\end{array}$

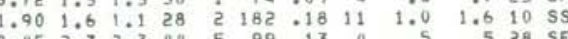

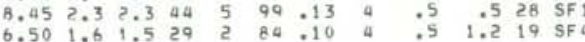
$\begin{array}{rrrrrrrrrrrrr}6.11 & 1.5 & 1.3 & 36 & 4 & 58 & 14 & 10 & .4 & 1.8 & 27 & \mathrm{GLN} \\ 8.21 & 2.8 & 2.5 & 38 & 2 & 100 & .20 & 7 & .6 & 1.2 & 29 & \mathrm{LSW}\end{array}$

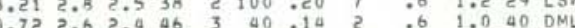

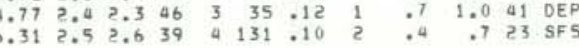

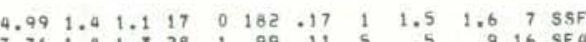

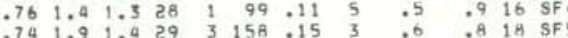

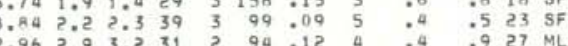

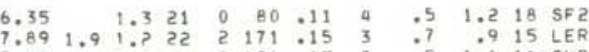

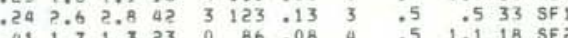

HVO EARTHQUAKE SUMMARY LIST

PAGE 32 ORIGIN TIME LAT N LONW DEPTH AMP DUR GAP RMS MIN ERH ERZ NO

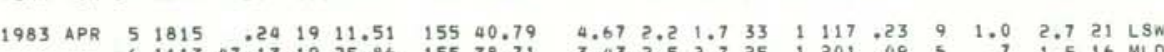

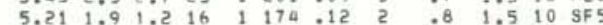

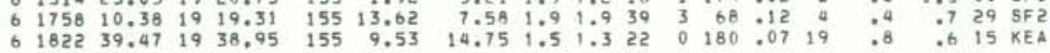

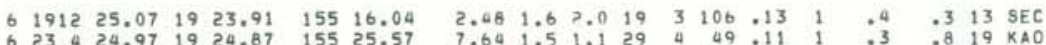

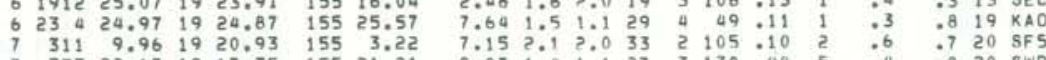

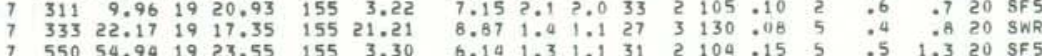

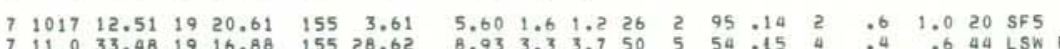

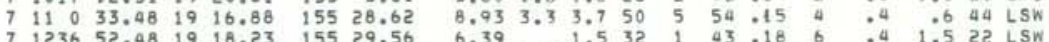

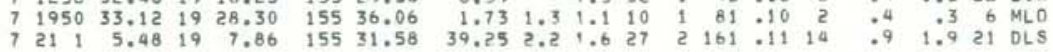

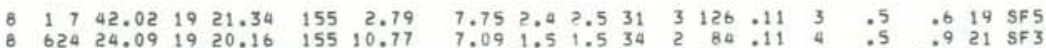

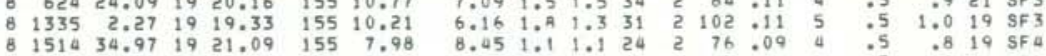

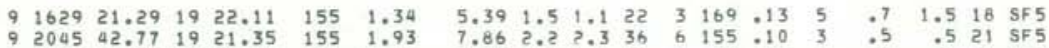

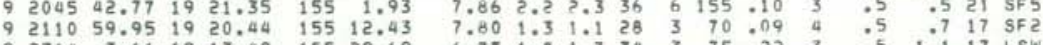

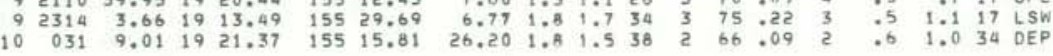

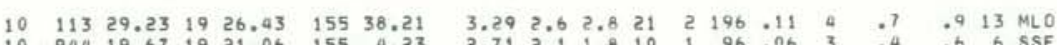

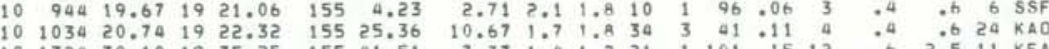

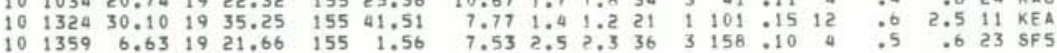

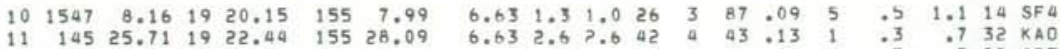

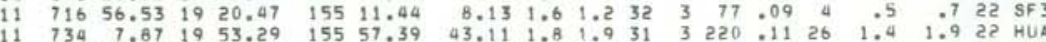

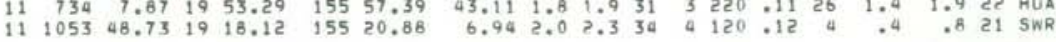
$\begin{array}{lllllll}11 & 1214 & 39.75 & 19 & 19.45 & 155 & 15.52\end{array}$

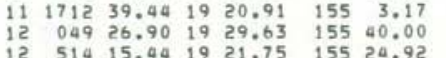
$\begin{array}{llllllll}5.98 & 1.0 & 1.3 & 29 & 2 & 97 & -11 \\ 2.84 & 2.5 & 0.7 & 19 & 3 & 200 & 009\end{array}$

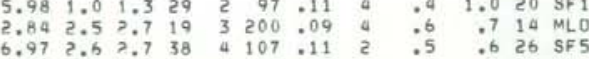

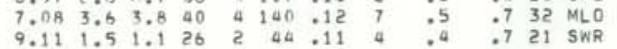

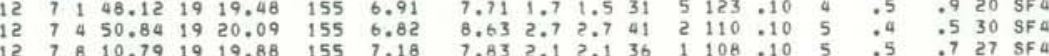

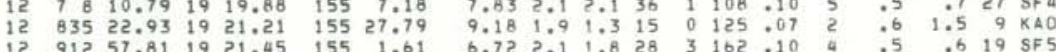

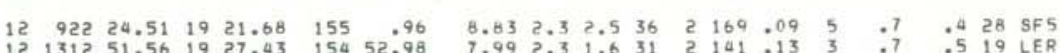

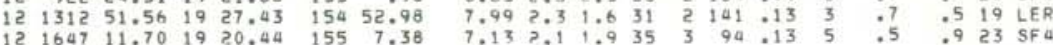


HVO EARTHQUAKE SUMMARY LIST

PAGE 33

$\begin{array}{llll}\text { ORIGIN TIME LATN } & \text { LON W } & \text { DEPTH AMP RIR } & \text { GAP RMS MIN ERH ERZ NO } \\ \text { OA HRMN SEE DEG MIN OEG MIN } & \text { KM MAG MAG NR NS DEG SEC OIS KM KM FM REMK }\end{array}$

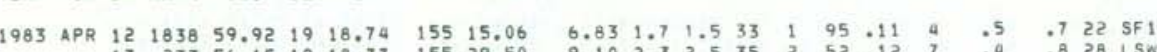

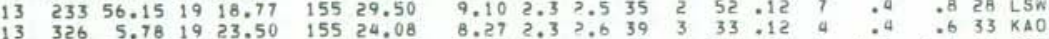

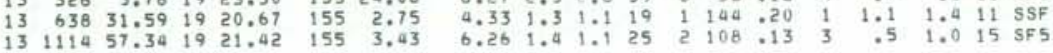

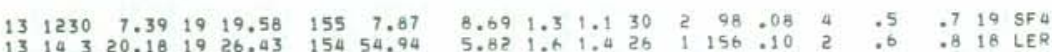

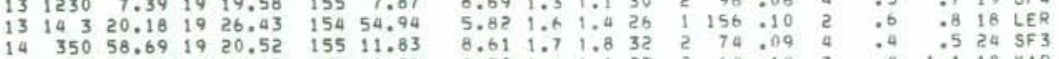

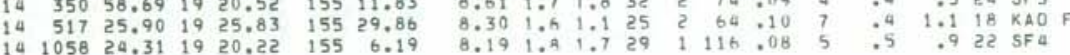

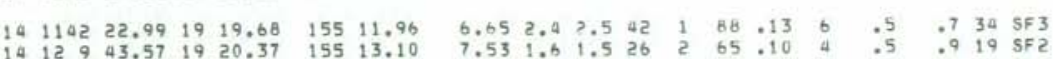

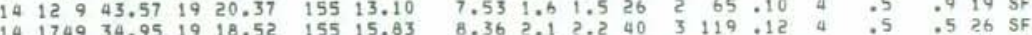

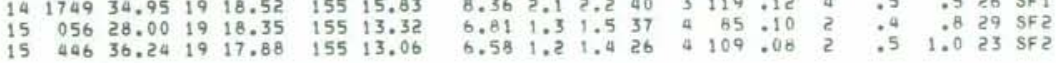

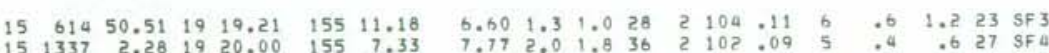

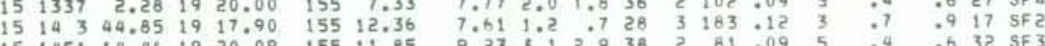

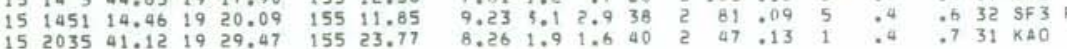

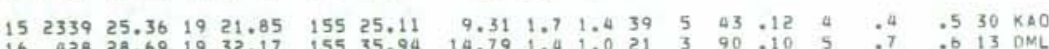

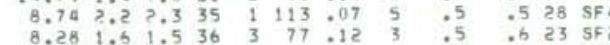

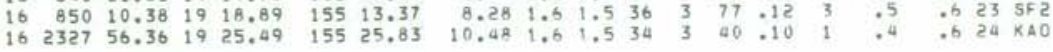
$\begin{array}{llllllllllllllllllll}16 & 2343 & 49.90 & 19 & 18.90 & 155 & 2.78 & 41.59 & 2.5 & 2.4 & 38 & 0 & 203 & 09 & 2 & 1.3 & 2.0 & 30 & \text { OEP } \\ 17 & 230 & 16.36 & 19 & 23.08 & 155 & 2.50 & 7.45 & 1.5 & 4 & 25 & 1 & 128 & 0.15 & 6 & 1.7 & 1.2 & 14 & \text { SF }\end{array}$

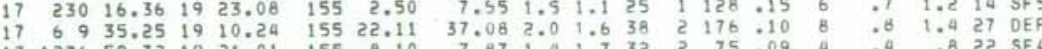

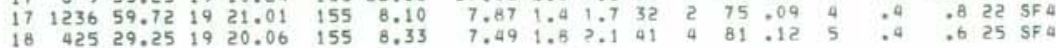

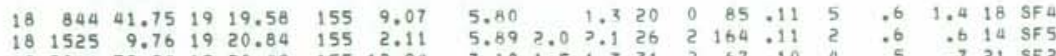

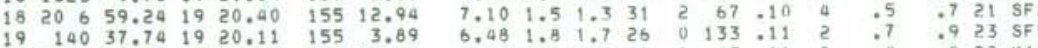

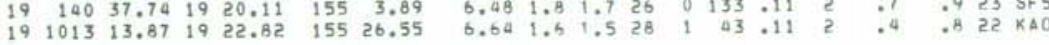

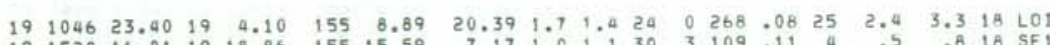

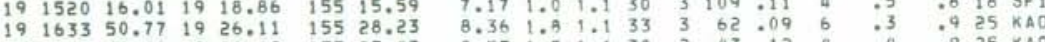

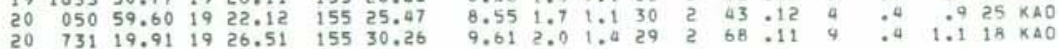
$\begin{array}{llllllllllllllllllll}20 & 1235 & 29.76 & 19 & 15.33 & 155 & 22.00 & 8.48 & 1.9 & 1.5 & 25 & 2 & 184 & .09 & 4 & .6 & 1.0 & 19 & \text { SWR } \\ 20 & 1641 & 26.57 & 19 & 6.56 & 155 & 28.69 & 30.33 & 1.4 & 1.5 & 30 & 0 & 240 & 08 & 6 & 1.9 & 2.2 & 25 & 0 L 5\end{array}$

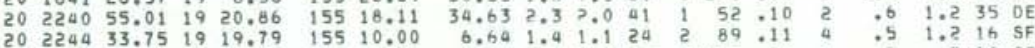

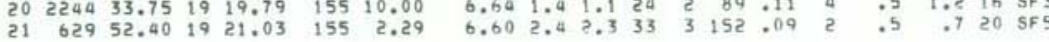

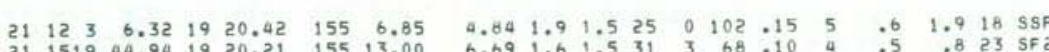

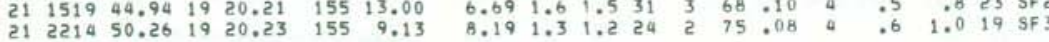

MVO EARTHOUAKE SUMMARY LIST

PAGE 34 ORIGIN TIME LAT N
OA HRMN SEN DE

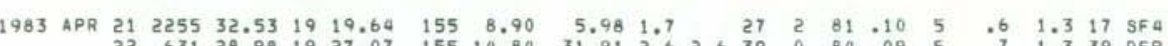

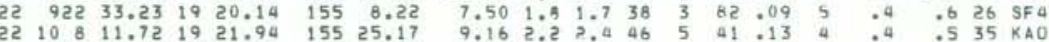

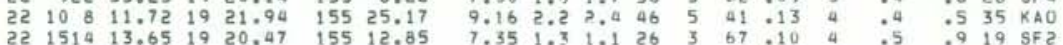

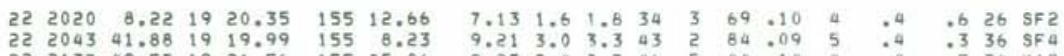

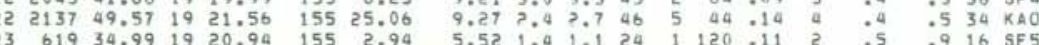

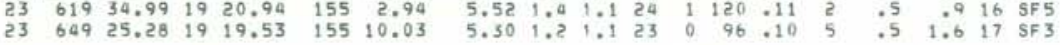

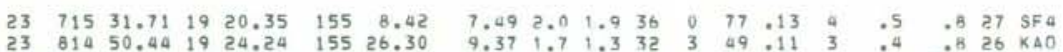

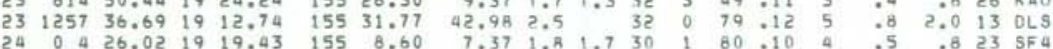

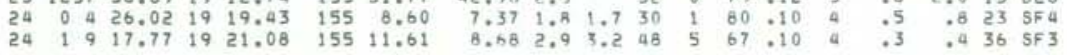

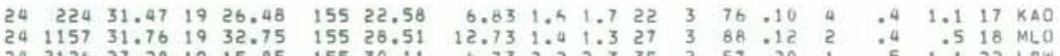

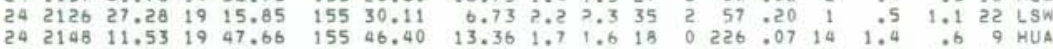

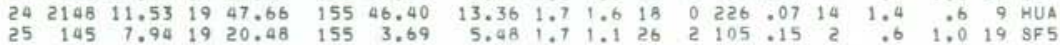

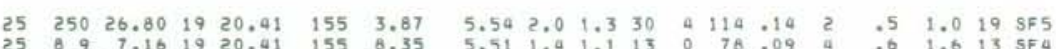

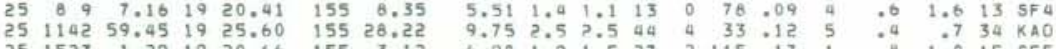

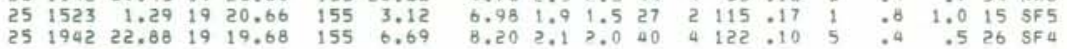
25 2058 $54.00 \quad 1920.82 \quad 155 \quad 3.40$

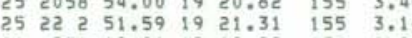
$26 \quad 336$ 4 $26 \quad 630 \quad 49.80 \quad 19 \quad 17.80 \quad 155,23.75$ $\begin{array}{lllllll}26 & 1431 & 29.76 & 19 & 20.09 & 155 & 7.18\end{array}$

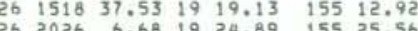

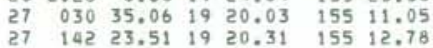

$\begin{array}{lllllll}97 & 934 & 4.03 \quad 20 & 23.70 & 156 & 17.98\end{array}$

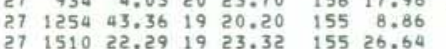

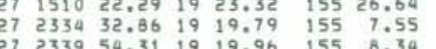
$\begin{array}{lllllll}27 & 2346 & 1.59 & 19 & 20.12 & 155 & 8.25 \\ 28 & 515 & 59.72 & 19 & 19.16 & 155 & 9.74\end{array}$

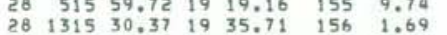
$\begin{array}{lllllll}28 & 1945 & 46.97 & 19 & 26.30 & 155 & 27.64 \\ 28 & 2250 & 27.60 & 19 & 20.40 & 155 & 7.07\end{array}$ $29 \quad 55959.63 \quad 19 \quad 19.70 \quad 155 \quad 18.60$

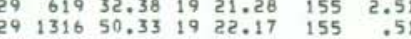

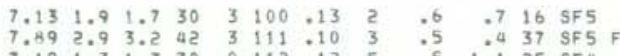

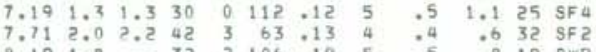

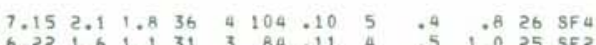

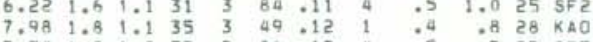
$\begin{array}{lllllllllllll}7.34 & 1.9 & 1.8 & 37 & 2 & 86 & 12 & 4 & : 5 & .7 & 27 & \mathrm{SF} 3 \\ 7.06 & 1.6 & 1.6 & 36 & 2 & 70 & : 11 & 4 & .5 & .7 & 25 & \mathrm{5F} 2\end{array}$ $\begin{array}{llllllllllll}25.61 & 3.3 & 3.4 & 38 & 4 & 207 & .13 & 42 & .8 & 3.0 & 32 & \mathrm{KOH}\end{array}$

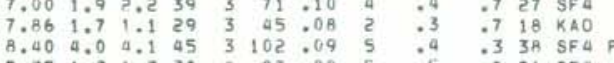

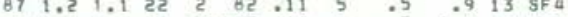

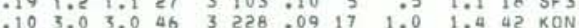
$\begin{array}{lllllllllllll}8.29 & 2.0 & 1.7 & 38 & 3 & 55 & .12 & 5 & .4 & .8 & 23 & K A 0 \\ 8.11 & 2.1 & 2.1 & 36 & 1 & 99 & .09 & 5 & .5 & .7 & 30 & 9 F 4\end{array}$ \begin{tabular}{llllllllllllll}
4.02 & 1.4 & 1.1 & 23 & 3 & 63 & .10 & 2 & .4 & .7 & 14 & SWR \\
\hline
\end{tabular}

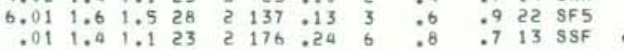


ORIGIN TIME LAT N LON K
DA HRMN SEPTH AMP MIIR NEG GAP RMS MIN ERH ERZ NO
DEG MEG MIN

1983 APR $29 \begin{array}{llllllllllllllllll}1835 & 35.95 & 19 & 13.29 & 155 & 27.98 & 7.23 & 2.5 & 2.4 & 43 & 3 & 101 & .19 & 5 & .5 & .9 & 30 & \text { LSWF }\end{array}$

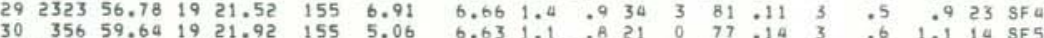

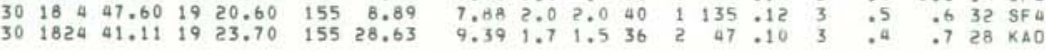

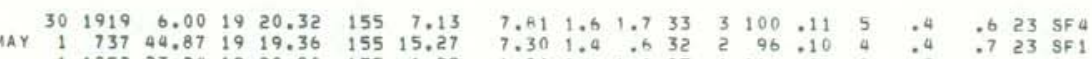

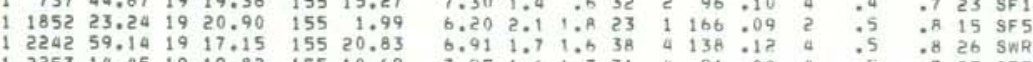

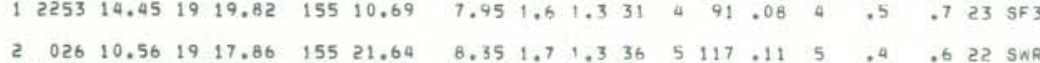

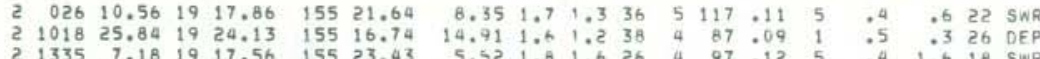

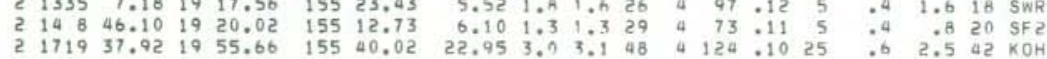

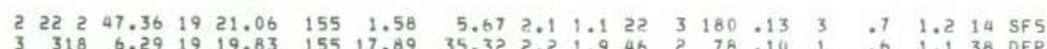

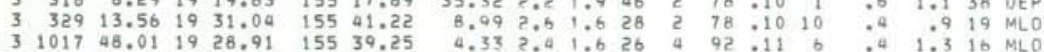

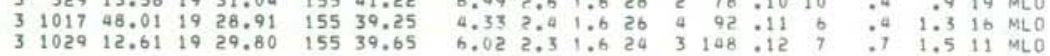

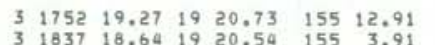

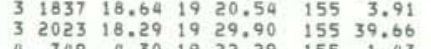
$4 \quad 824 \quad 30.70 \quad 19 \quad 19.10 \quad 15514.04$ 4) $1648 \quad 32.97 \quad 1920.30 \quad 155 \quad 13.22$

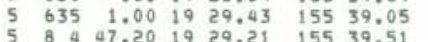

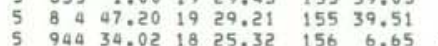
$\begin{array}{lllllll}5 & 1628 & 50.17 & 19 & 16.65 & 155 & 13.29 \\ 5 & 1638 & 30.75 & 19 & 16.21 & 155 & 13.24\end{array}$ 51780.861916 .9515513 .22 $51826 \quad 26.691919 .73 \quad 155,9.46$ $52128 \quad 55.92 \quad 1920.19 \quad 155 \quad 10.59$

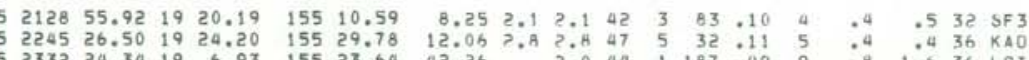

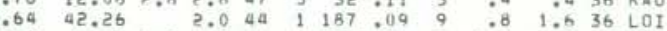

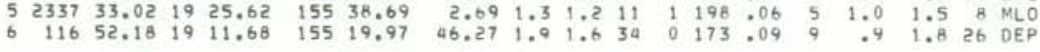

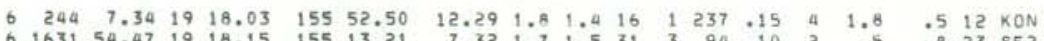

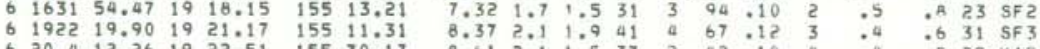

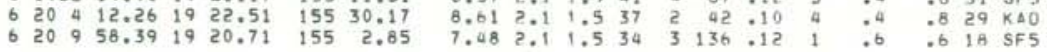

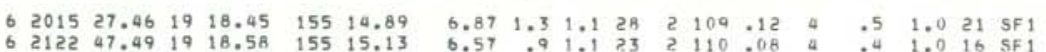

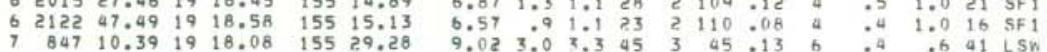

ORIGIN TIME LAT N LON W DEPTH AMP RUR GAP RMS MIN ERH ERZ NO

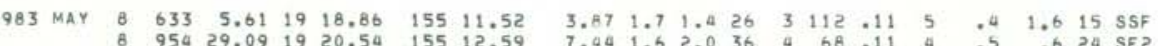

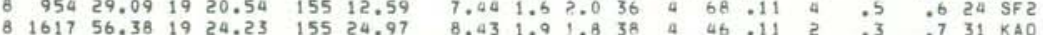

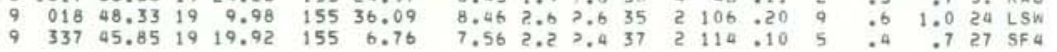

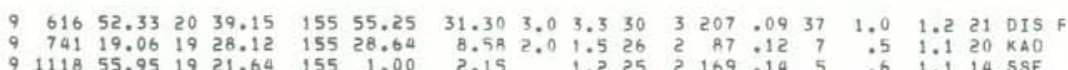

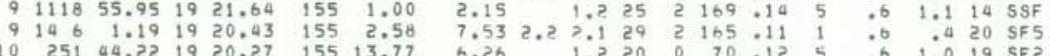

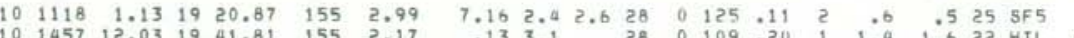

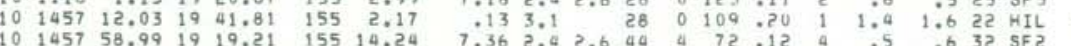

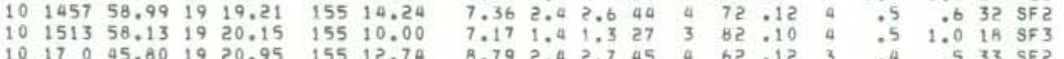

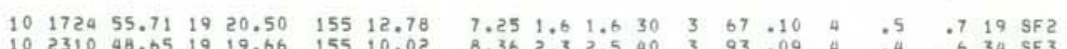

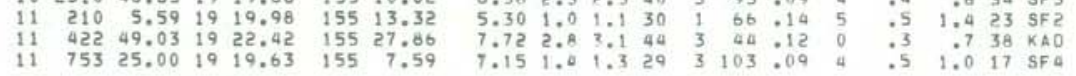

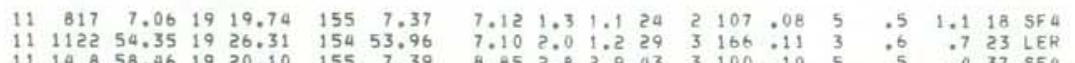

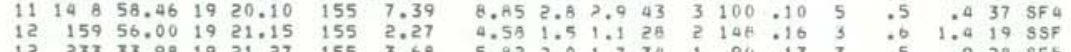

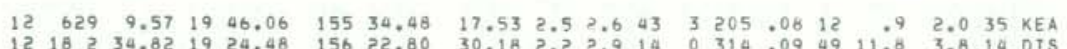

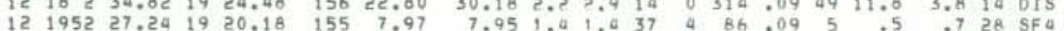
$\begin{array}{lllllllllllllllllll}12 & 1953 & 18.24 & 19 & 20.37 & 155 & 7.80 & 7.73 & 1.8 & : 7 & 37 & 3 & 88 & 10 & 5 & .5 & .7 & 29 & \text { SF } \\ 12 & 2152 & 28.07 & 19 & 24.93 & 155 & 15.65 & 38.74 & 1.4 & 1.9 & 27 & 0 & 91 & .08 & 2 & 1.0 & 2.5 & 18 & \text { DEP }\end{array}$

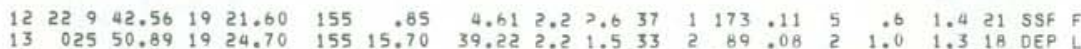

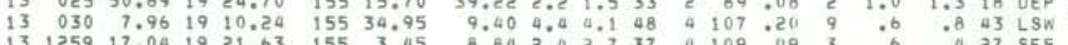

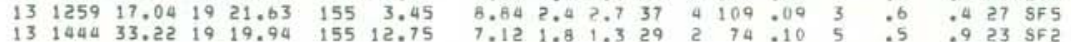

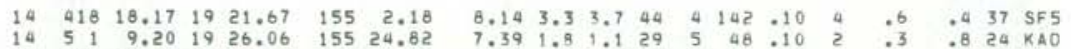

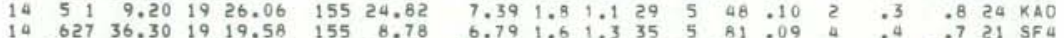

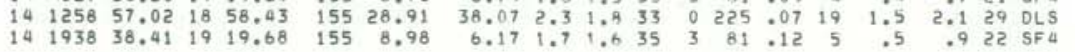
$\begin{array}{lllllllllllllllllll}15 & 4 & 6 & 38.89 & 19.24 .59 & 155 & 29.83 & 7.85 & 2.4 & 2.2 & 39 & 3 & 53 & 11 & 5 & .3 & .0 & 28 & \text { KAO }\end{array}$

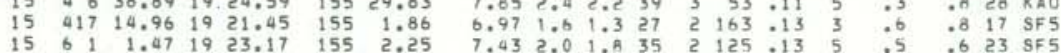

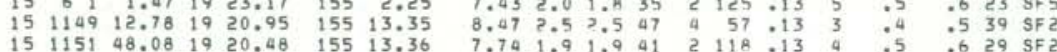

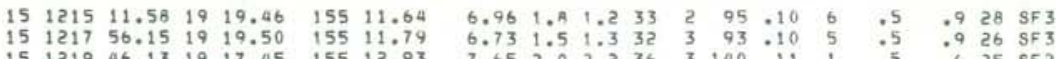


HVO EARTHQUAKE SUMMARY LIST

PAGE 37

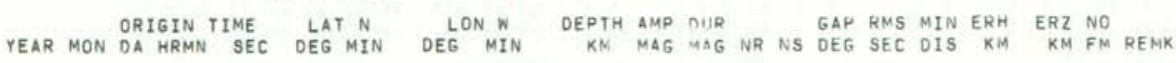

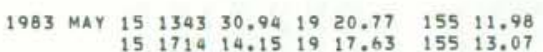
$\begin{array}{llllllll}5 & 21 & 4 & 24.43 & 19 & 21.76 & 155 & 5.0\end{array}$

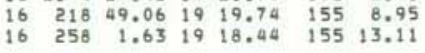

$\begin{array}{rrrrrrr}16 & 743 & 17.75 & 19 & 19.40 & 155 & 11.49 \\ 16 & 1051 & 31.37 & 19 & 18.78 & 155 & 30.51\end{array}$

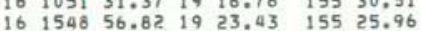

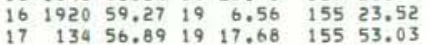

$\begin{array}{lllllll}17 & 411 & 53.83 & 19 & 19.39 & 155 & 9.94\end{array}$ $17 \quad 427 \quad 10.08 \quad 1924.06 \quad 15526.05$

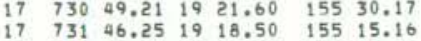
$17 \quad 75136.001921 .52 \quad 155 \quad 30.06$

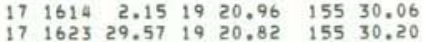
$17162329.571920 .82 \quad 15530.20$

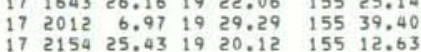

$\begin{array}{lllllll}18 & 346 & 54.66 & 19 & 10.24 & 155 & 32.49\end{array}$

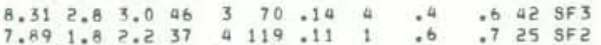

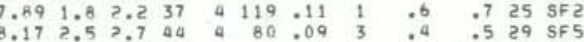

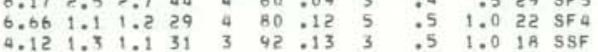

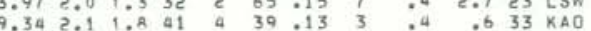

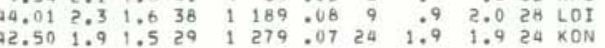
$\begin{array}{llllllllllll}6.23 & 1.2 & 1.1 & 26 & 2 & 98 & .09 & 5 & .5 & 1.2 & 15 & \text { SF } 3 \\ 1.70 & .6 & 1.3 & 31 & 0 & 47 & 10 & 3 & .4 & .8 & 26 & \text { KAO }\end{array}$

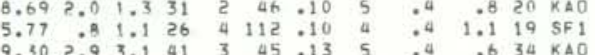
$\begin{array}{lllllllllllll}8.23 & 1.7 & 1.3 & 29 & 1 & 47 & .13 & 5 & .5 & 1.2 & 22 & k A 0 \\ 9.38 & 2.2 & 7.2 & 31 & 1 & 49 & 07 & 6 & .5 & .9 & 28 & k A 0\end{array}$ $\begin{array}{lllll}.11 & 4 & .4 & .7 & 23 \\ 12 & \text { KAO }\end{array}$

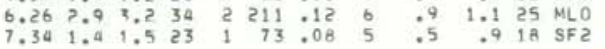

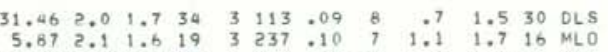
$\begin{array}{lllllllllllll}8.76 & 1.6 & .5 & 32 & 2 & 98 & .12 & 6 & .5 & .8 & 25 & 5 F 3\end{array}$

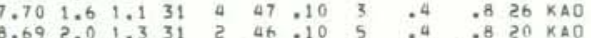

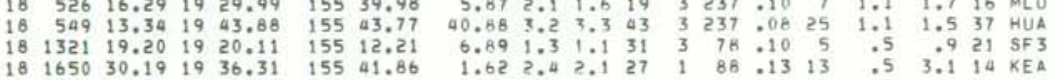

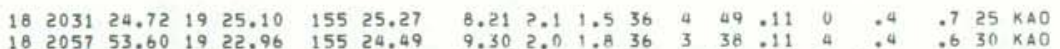

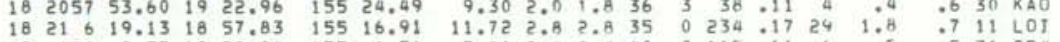

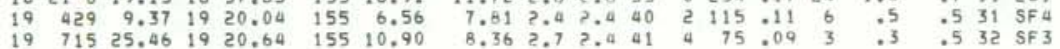

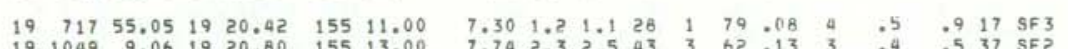

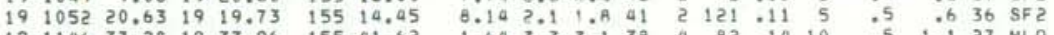

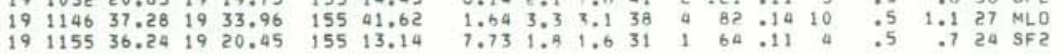
$\begin{array}{llllllllllllllllllll}19 & 1534 & 22.36 & 19 & 19.60 & 155 & 26.50 & 7.71 & 1.4 & 1.2 & 29 & 2 & 61 & .15 & 6 & .4 & 1.1 & 23 & \text { KAO } \\ 19 & 17 & 57.41 & 19 & 35.67 & 155 & 01.74 & 1.14 & 2.0 & 2.3 & 22 & 2 & 141 & 010 & 12 & : 7 & 1.0 & 17 & \text { KEA }\end{array}$

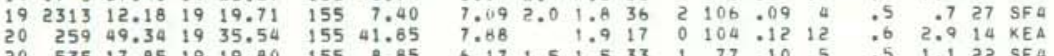
$\begin{array}{lllllllllllll} & 6.17 & 1.5 & 1.5 & 33 & 1 & 77 & .10 & 5 & .5 & 1.1 & \text { 2.2 SF } & \text { SF }\end{array}$

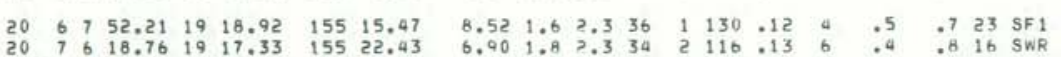

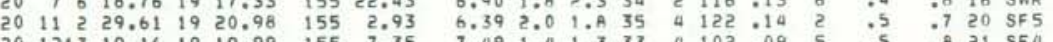

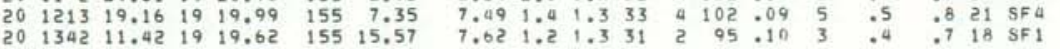

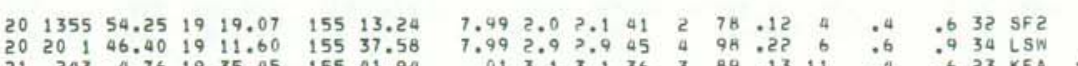

HVO EARTHQUAKE SUMMARY LIST

PAGE 38

ORIGIN TIME LAT N LON W DEPTH AMP NUR GAP RMS MIN ERH ERZ NO

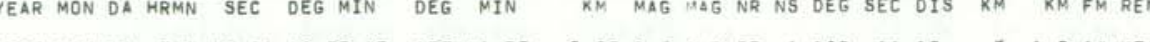

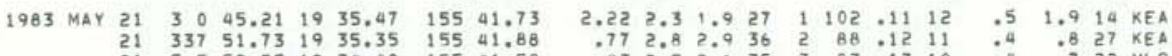

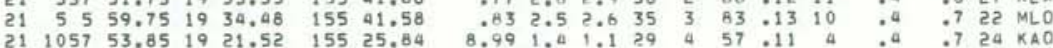

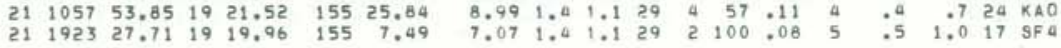

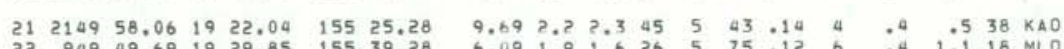

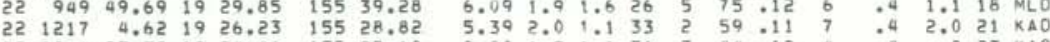

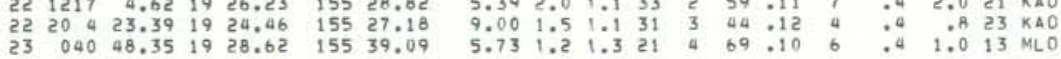

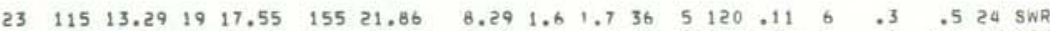

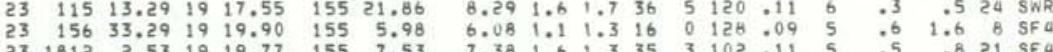

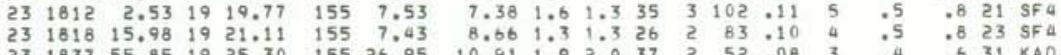

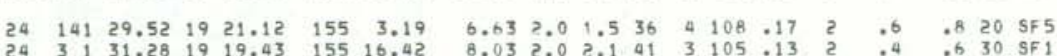

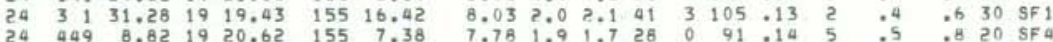

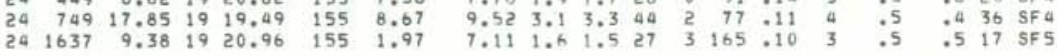

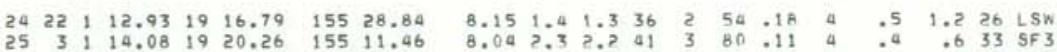

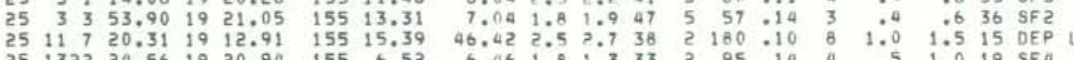

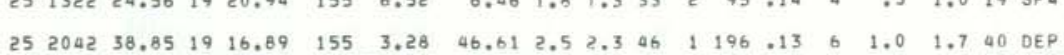

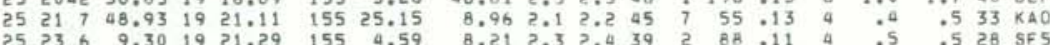

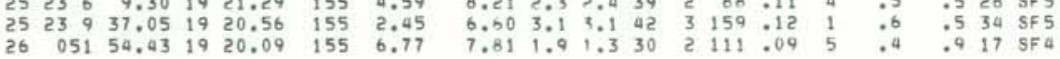
$\begin{array}{llllllllllllllllll}26 & 913 & 5.24 & 20 & 10.08 & 155 & 6.31 & 10.08 & 2.0 & 11 & 0 & 269 & .09 & 40 & 5.6 & 3.7 & 5 & \text { KEA }\end{array}$

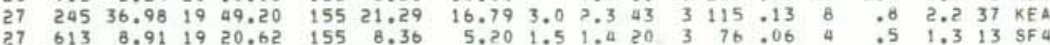

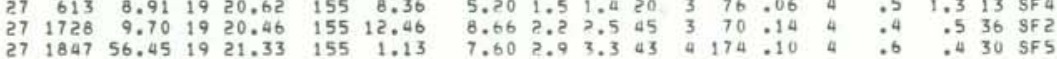
$\begin{array}{llllllllllllllllllll}27 & 1919 & 46.88 & 19 & 11.44 & 155 & 29.08 & 33.23 & 2.4 & 1.5 & 40 & 1 & 75 & 08 & 4 & .6 & 1.5 & 34 & \text { OLS } \\ 27 & 2040 & 45.32 & 19 & 38.23 & 156 & 28.52 & 24.89 & 2.3 & 2.7 & 16 & 0 & 283 & .12 & 61 & 5.0 & 8.0 & 10 & 01 S\end{array}$

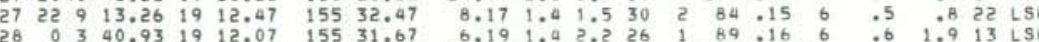

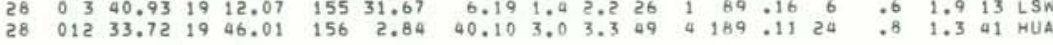
$\begin{array}{lllllllllllllllllllll}28 & 542 & 10.30 & 19 & 20.41 & 155 & 12.65 & 7.32 & 1.5 & 1.4 & 30 & 2 & 69 & .10 & 4 & .5 & .9 & 22 & \text { SF } \\ 28 & 548 & 11.94 & 19 & 21.15 & 155 & 6.02 & 4.80 & 1.9 & 1.3 & 26 & 0 & 93 & .12 & 6 & .5 & 3.0 & 18 & \text { SSF }\end{array}$

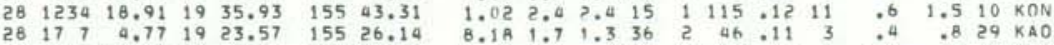

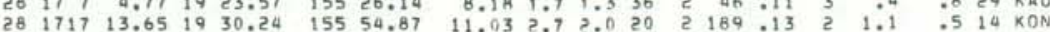

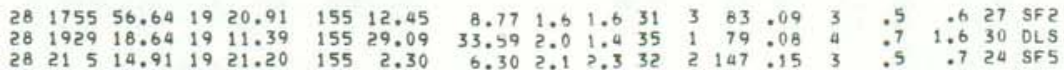




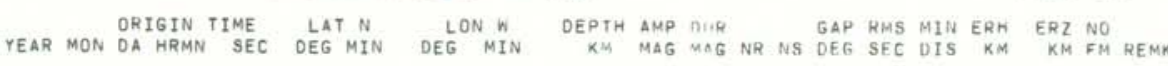

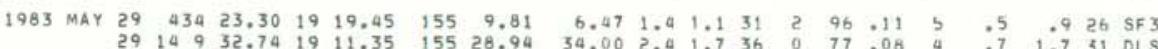

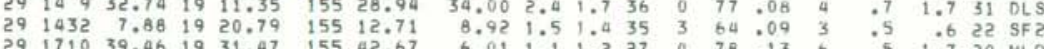

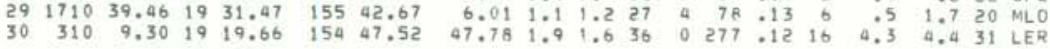
$\begin{array}{lllllll}30 & 1045 & 32.94 & 19 & 19.48 & 155 & 10.38 \\ 30 & 1232 & 31.78 & 19 & 22.00 & 155 & 2.95\end{array}$

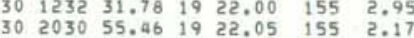

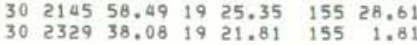

$\begin{array}{lllllll}31 & 211 & 44.32 & 19 & 19.53 \quad 155 & 8.03\end{array}$

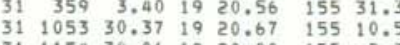

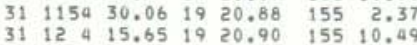

$\begin{array}{rrrrrrr}31 & 2040 & 5.58 & 19 & 26.89 & 155 & 29.50\end{array}$ $14931.851920 .76 \quad 155 \quad 2.40$

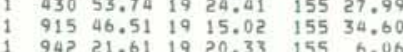
$\begin{array}{llllllll}1 & 13 & 6 & 16.47 & 19 & 22.06 & 155 & 25.49\end{array}$ $\begin{array}{rrrrrr}15 & 12.94 & 19 & 19.91 & 155 & 4.97 \\ 1543 & 47.12 & 19 & 18.57 & 155 & 14.87\end{array}$

$\begin{array}{lllllll}1 & 1853 & 32.45 & 19 & 11.53 & 155 & 29.06 \\ 1 & 2049 & 11.92 & 19 & 19.58 & 155 & 11.72\end{array}$

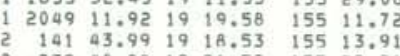
$\begin{array}{lllllll}2 & 141 & 43.99 & 19 & 18.53 & 155 & 13.91 \\ 2 & 239 & 40.88 & 19 & 26.52 & 155 & 29.21 \\ 2 & 759 & 14.42 & 19 & 21.69 & 155 & 25.36\end{array}$ $\begin{array}{lllllll}2 & 1028 & 54.42 & 19 & 21.44 & 155 & 2.63\end{array}$

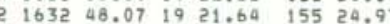

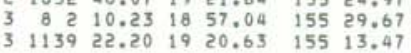

$3 \quad 1746 \quad 1.08 \quad 19 \quad 19.65 \quad 155 \quad 4.97$

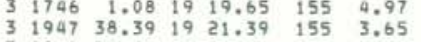

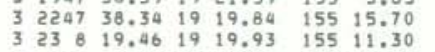

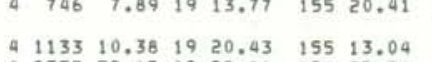

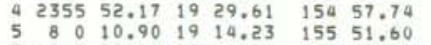

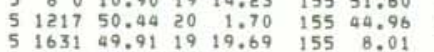
$\begin{array}{lllllll}5 & 1731 & 21.36 & 19 & 27.46 & 155 & 26.57 \\ 5 & 185 & 31.04 & 19 & 9.17 & 155 & 32.44 \\ 5 & 19 & 26.04\end{array}$

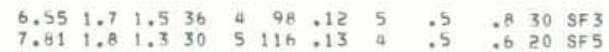

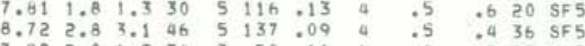

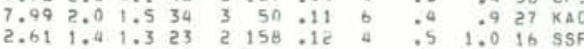

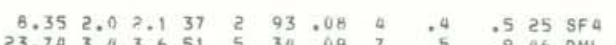

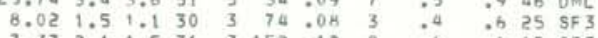

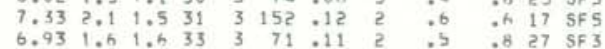

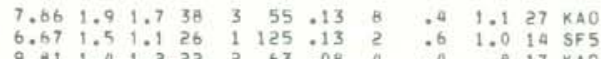

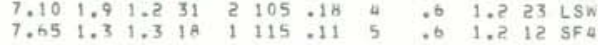

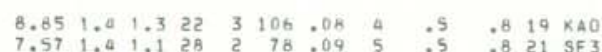

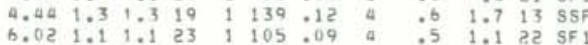

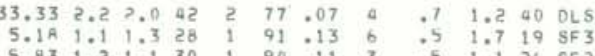

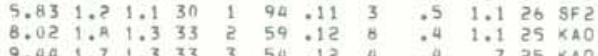

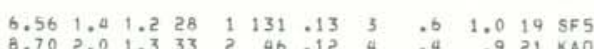

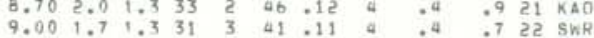

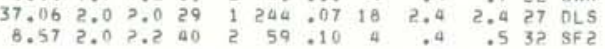
$\begin{array}{llllllllllll}4.92 & 1.1 & 1.1 & 16 & 1 & 149 & .23 & 4 & 1.2 & 3.6 & 12 & \text { SSF }\end{array}$

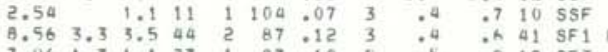

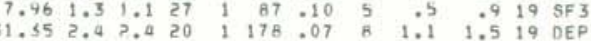
\begin{tabular}{rrrrrrrrrrrrrr}
7.35 & 1.2 & 1.1 & 31 & 2 & 65 & 10 & 4 & .5 & .7 & 20 & SF 2 \\
43.94 & 3.5 & 7.7 & 36 & 0 & 102 & 11 & 6 & 1.6 & 2.8 & 33 & LER \\
\hline
\end{tabular}

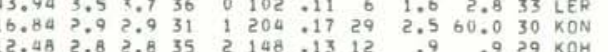

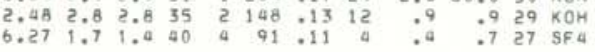

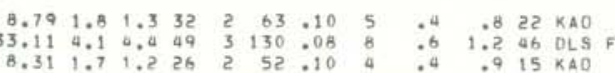

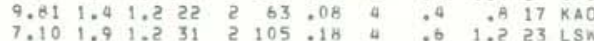

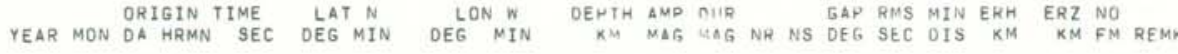

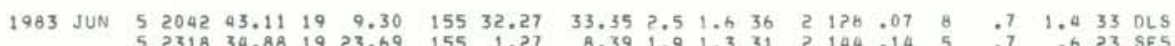

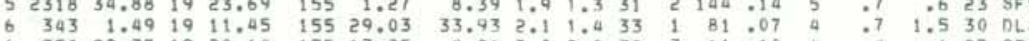

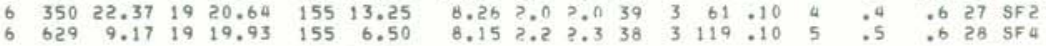

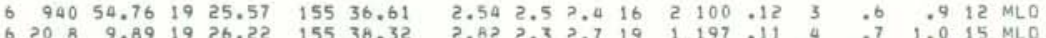

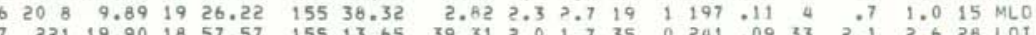

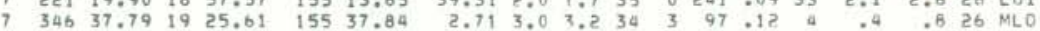

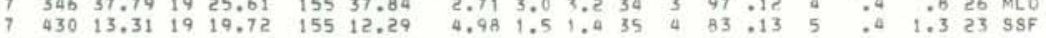

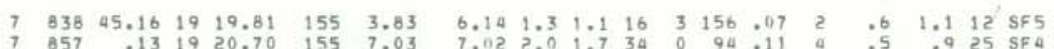

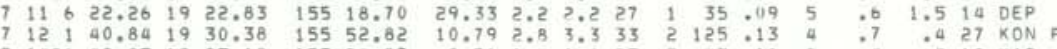

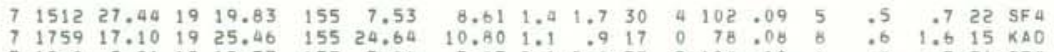

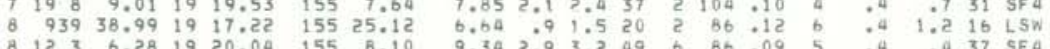

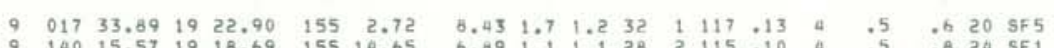

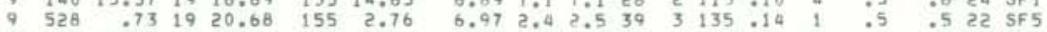

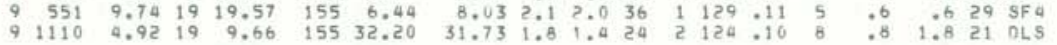

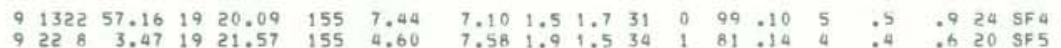

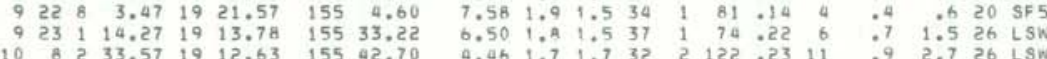

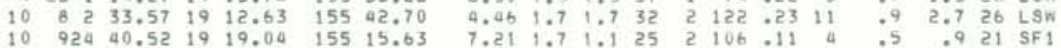

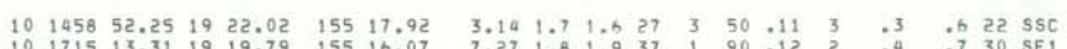

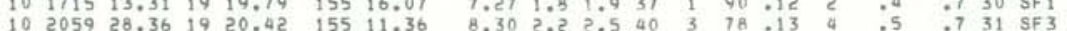

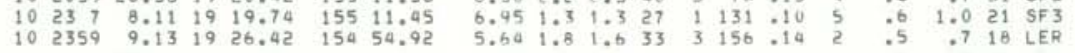

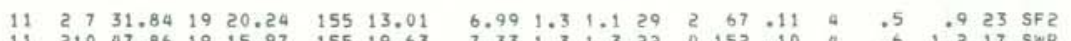

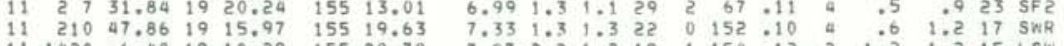

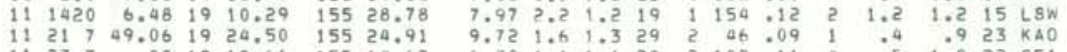
$\begin{array}{llllllllllllllllllll}12 & 0 & 6 & 10.38 & 19 & 19.52 & 155 & 13.12 & 8.08 & 1.5 & 1.5 & 36 & 3 & 75 & .11 & 5 & .5 & .7 & 26 & \text { SF }\end{array}$

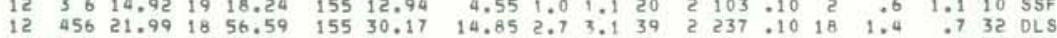

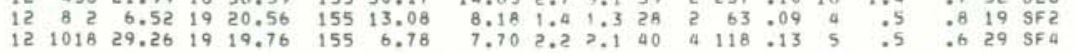

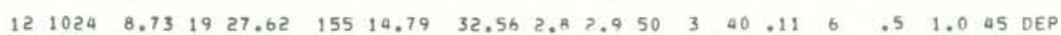

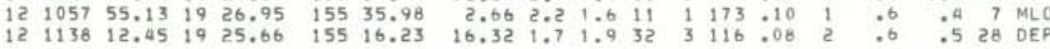


HVO EARTHQUAKE SUMMARY LIST

PAGF 41

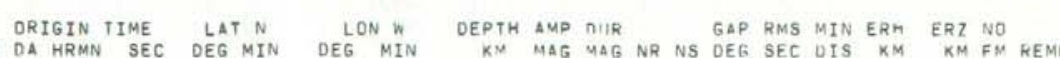

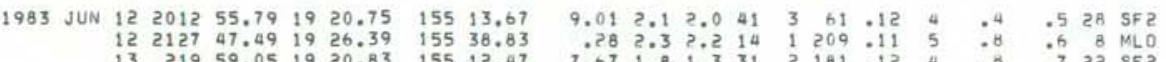
13 219 59.05 19 $20.33 \quad 155$ 30.03

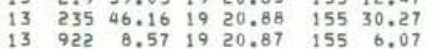

$\begin{array}{lllllll}13 & 1245 & 27.19 & 19 & 26.67 & 155 & 24.34\end{array}$

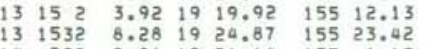

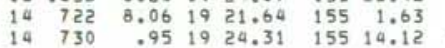

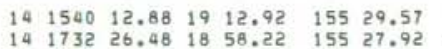

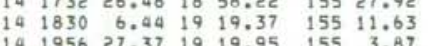
$\begin{array}{lllllll}14 & 1956 & 27.37 & 19 & 19.95 & 155 & 3.87 \\ 14 & 22288 & 30.80 & 19 & 19.74 & 155 & 11.80\end{array}$ $\begin{array}{lllllll}14 & 2353 & 23.77 & 19 & 21.21 & 155 & 4.68\end{array}$ $15 \quad 633 \quad 54.28 \quad 19 \quad 18.61 \quad 155 \quad 13.65$

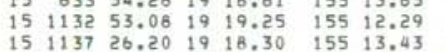

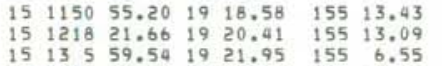

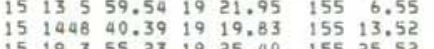

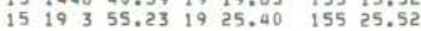
$\begin{array}{llllllll}16 & 0 & 1 & 8.73 & 19 & 20.74 & 155 & 13.22\end{array}$

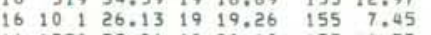

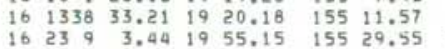
$\begin{array}{lllllll}17 & 152 & 55.27 & 19 & 18.99 & 155 & 3.62\end{array}$

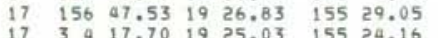

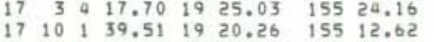
\begin{tabular}{l}
$17 \quad 1046 \quad 37.15 \quad 1953.18 \quad 15531.28$ \\
\hline
\end{tabular}

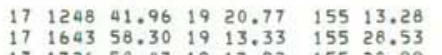

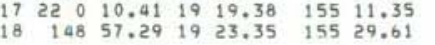
$\begin{array}{lllllll}18 & 151 & 23.81 & 19 & 24.97 & 155 & 16.01 \\ 18 & 731 & 54.99 & 19 & 20.04 & 155 & 7.04\end{array}$

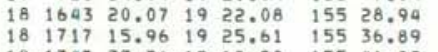
$\begin{array}{lllllll}19 & 142 & 26.42 & 19 & 16.26 & 155 & 23.26 \\ 19 & 748 & 41.95 & 19 & 20.28 & 155 & 7.29\end{array}$ $\begin{array}{rrrrrrr}19 & 748 & 41.95 & 19 & 20.28 & 155 & 7.29 \\ 19 & 1335 & 48.97 & 19 & 16.08 & 155 & 21.98\end{array}$

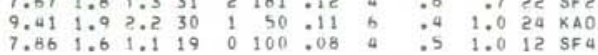

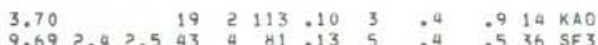

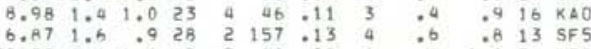
$\begin{array}{llllllllllllll}8.06 & 2.3 & 2.1 & 37 & 2 & 125 & .19 & 4 & .6 & .9 & 23 & \text { L.5W }\end{array}$

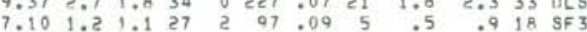

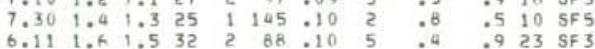

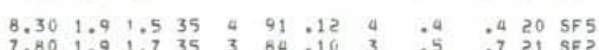

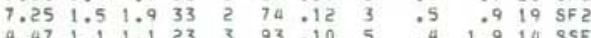
$\begin{array}{rlllllllllll}7.25 & 1.2 & 1.1 & 22 & 1 & 78 & 10 & 3 & .5 & 1.2 & 16 & \text { SF } 2 \\ 6.51 & 1.5 & 1.3 & 26 & 2 & 64 & 010 & 4 & .5 & .0 & 16 & \text { SF } 2\end{array}$

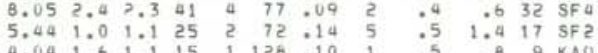
$\begin{array}{llllllllllllllll}7.52 & 1.2 & 1.1 & 30 & 2 & 60 & .10 & 4 & .5 & .7 & 19 & \text { SF2 }\end{array}$

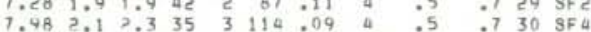

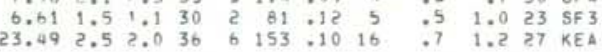
$\begin{array}{lllllllllllll}7.22 & 2.8 & 3.3 & 39 & 3 & 194 & .09 & 9 & .7 & .6 & 29 & 5 F 5\end{array}$

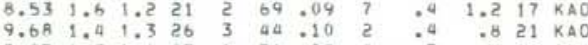
$\begin{array}{llllllllllll}7.55 & 2.0 & 7.3 & 44 & 6 & 59 & .15 & 3 & .4 & .6 & 29 & 5 F 2 \\ .00 & 2.6 & 2.8 & 45 & 5 & 92 & .15 & 7 & .3 & .4 & 23 & \mathrm{LSN}\end{array}$

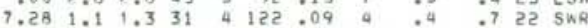
$\begin{array}{llllllllllll}5.27 & 1.2 & 1.1 & 28 & 5 & 99 & 11 & 6 & 04 & 1.4 & 17 & \text { SF } 3 \\ 9.52 & 1.9 & 1.3 & 36 & 4 & 47 & 07 & 4 & : 3 & .7 & 22 & K \Delta 0\end{array}$ $\begin{array}{llllllllllll}15.22 & 1.4 & 1.2 & 38 & 4 & 73 & -11 & 5 & .5 & .3 & 57 & \text { OEP }\end{array}$

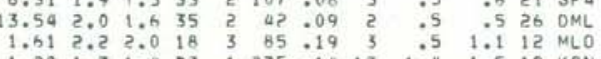

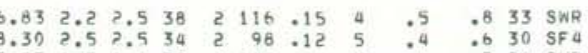

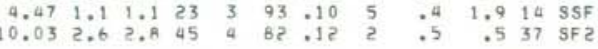

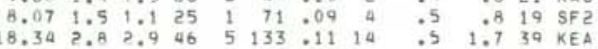

HVO EARTHQUAKE SUMMARY LIST

PAGE 42

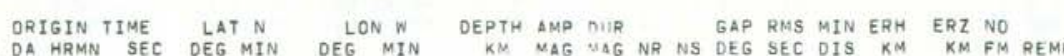
1983 JUN $19 \begin{array}{llllllllllllllllllllll}1711 & 16.71 & 19 & 13.41 & 155 & 31.39 & 5.54 & 2.2 & 1.1 & 27 & 2 & 71 & .23 & 4 & .7 & 1.6 & 17 & \text { LSW }\end{array}$

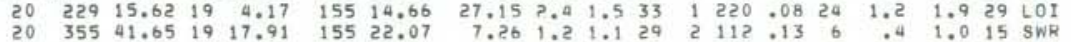

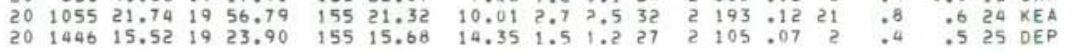

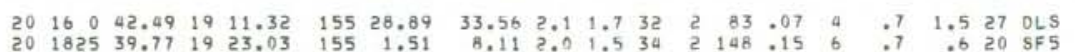

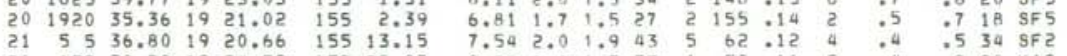

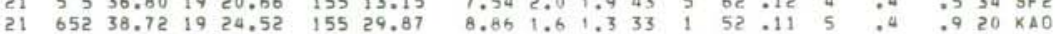
$\begin{array}{lllllllllllllllllll}21 & 754 & 47.69 & 19 & 28.48 & 155 & 37.35 & 1.01 & 2.4 & 2.4 & 28 & 2 & 101 & .17 & 3 & .4 & .5 & 17 \text { MLO } \\ 21 & 858 & 42.95 & 19 & 29.13 & 154 & 53.37 & .00 & 2.2 & 1.8 & 23 & 0 & 95 & .18 & 5 & .5 & 1.3 & 13 & \text { SLE . }\end{array}$

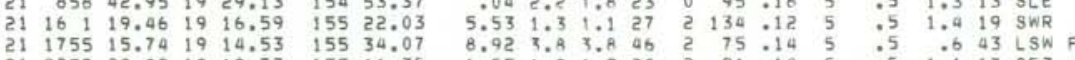

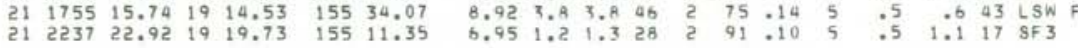

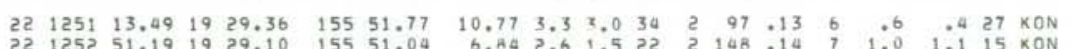

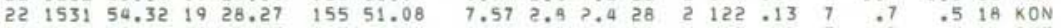

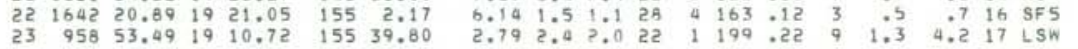
$\begin{array}{lllllllllllllllllllll}23 & 1910 & 14.93 & 19 & 6.86 & 155 & 23.52 & 43.13 & 2.0 & 1.8 & 32 & 0 & 187 & .08 & 9 & 1.0 & 2.9 & 32 & \text { LOI } \\ 23 & 2221 & 22.64 & 19 & 19.91 & 155 & 8.68 & 5.11 & 2.1 & 1.8 & 39 & 2 & 76 & .13 & 5 & .5 & 1.1 & 2 B & S F 4\end{array}$

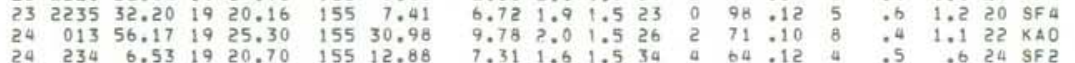

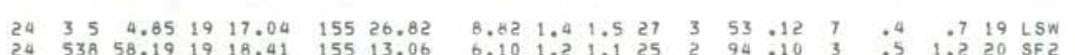
$24 \quad 832 \quad 41.531921 .65 \quad 1552.61 \quad 6.4022007 .129 \quad 2 \quad 130.16 \quad 3 \quad .6 \quad 1.023 \quad 955$

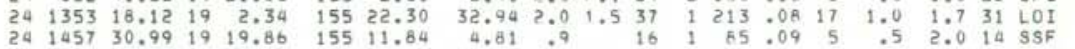

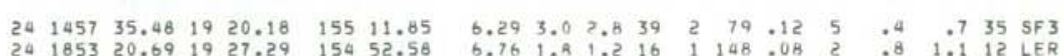

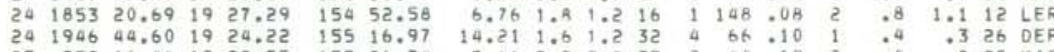

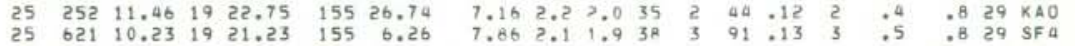
$\begin{array}{llllllllllllllllllll}25 & 659 & 9.26 & 19 & 17.46 & 155 & 37.54 & .25 & 2.1 & 2.0 & 19 & 0 & 159 & .11 & 5 & 1.0 & 3.1 & 15 & \text { LSW } \\ 25 & 1820 & 7.48 & 19 & 10.18 & 155 & 33.06 & 6.22 & 2.7 & 2.7 & 35 & 2 & 113 & .10 & 9 & .5 & .9 & 25 & \text { LSW }\end{array}$

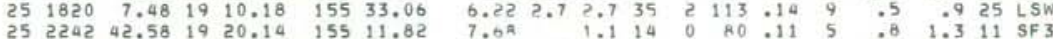

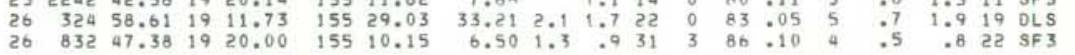

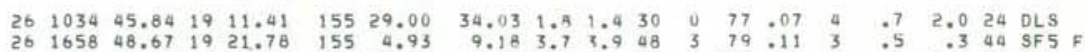

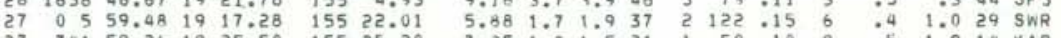

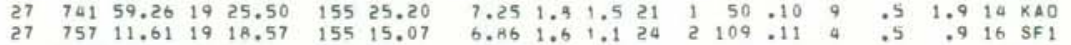
$\begin{array}{rrrrrrrrrrrrrrrrrrr}27 & 1131 & 2.09 & 19 & 19.23 & 155 & 9.99 & 7.04 & 1.4 & 1.3 & 28 & 2 & 103 & .09 & 5 & .5 & 1.1 & 19 & \text { SF } 3 \\ 27 & 1644 & 58.71 & 19 & 20.63 & 155 & 13.01 & 7.00 & 1.5 & 1.8 & 37 & 4 & 63 & .12 & 4 & .5 & .7 & 26 & \text { SF } 2\end{array}$ 
HVO EARTHQUAKE SUMMARY LIST

PAGE Q 3

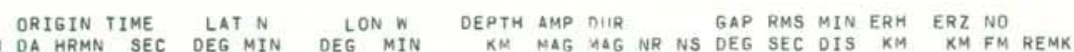

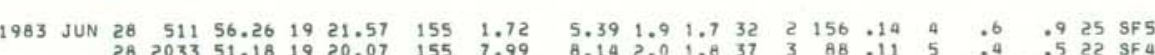

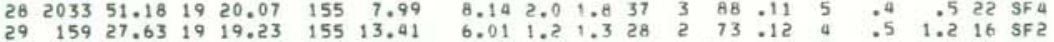

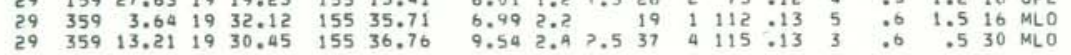

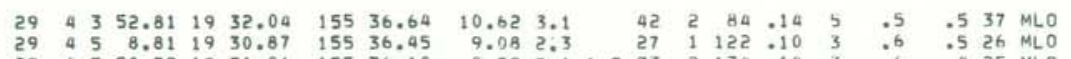

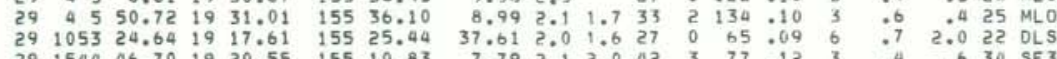
$\begin{array}{llllllllllllllllllll}29 & 2047 & 45.40 & 19 & 20.15 & 155 & 10.67 & 7.76 & 2.4 & 2.7 & 43 & 3 & 84 & .12 & 4 & .4 & .7 & 32 & 5 F 3\end{array}$

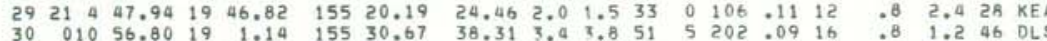

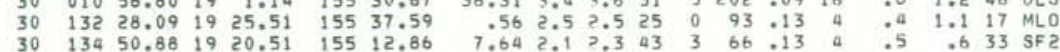
$\begin{array}{lllllllllllllllllll}30 & 136 & 9.80 & 19 & 20.69 & 155 & 6.91 & 8.64 & 2.7 & 3.0 & 44 & 3 & 96 & 10 & 4 & .4 & .4 & 32 & 5 F 4\end{array}$

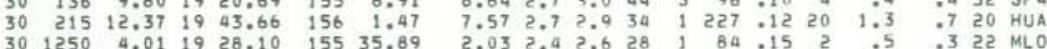

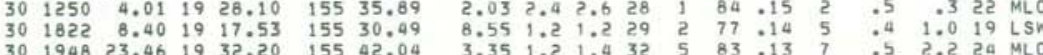

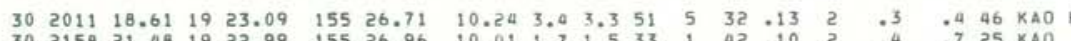

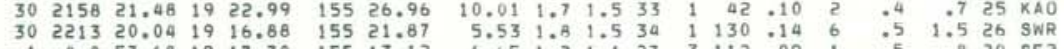

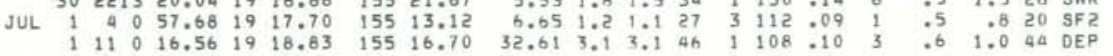

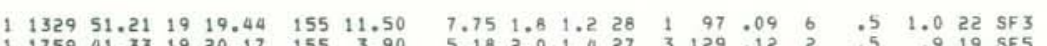

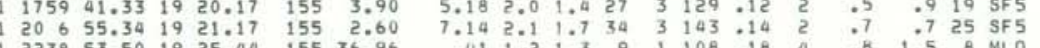

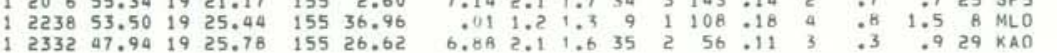

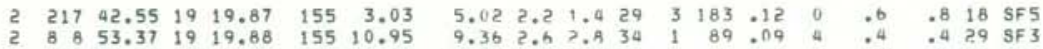

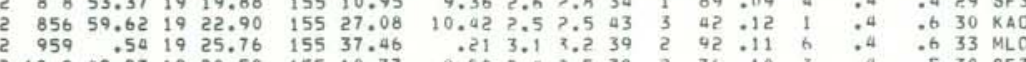

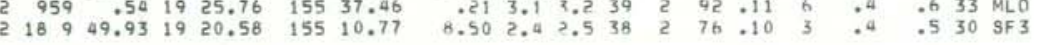

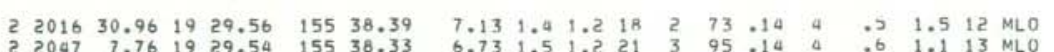

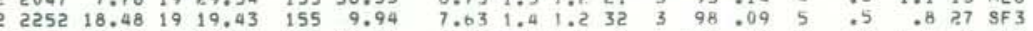

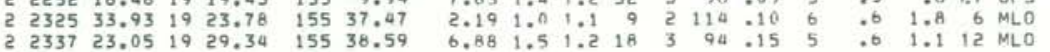

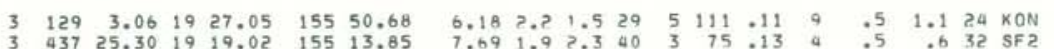

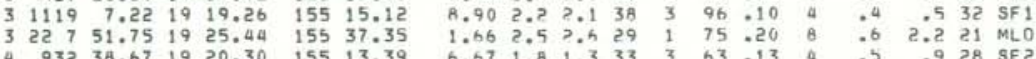
$\begin{array}{llllllllllllllllllll}4 & 1414 & 4.43 & 19 & 24.24 & 154 & 58.83 & 7.02 & 2.1 & 1.8 & 35 & 2 & 166 & .13 & 2 & .7 & .7 & 24 & \text { LER }\end{array}$

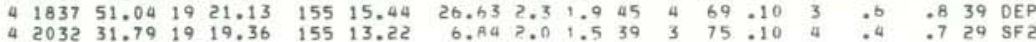

HVO EARTHQUAKE SUMMARY LIST

PAGE 44 ORIGIN TIME
DA HRMN SAT N

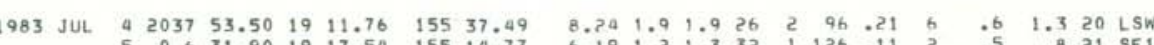

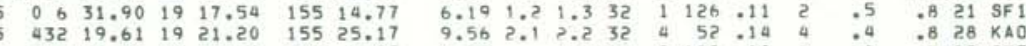

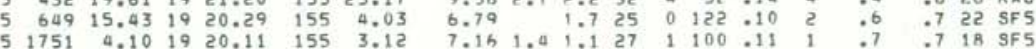

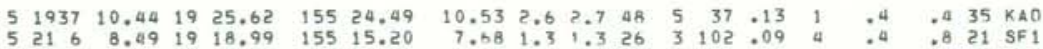

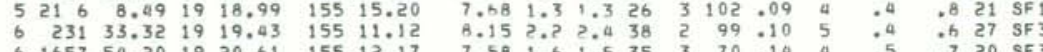

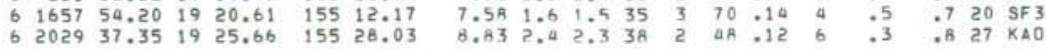

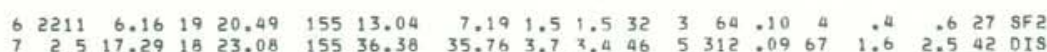

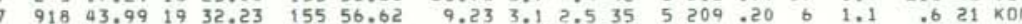

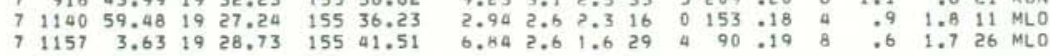

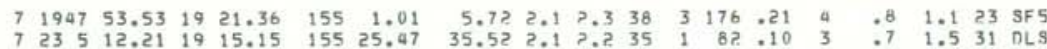

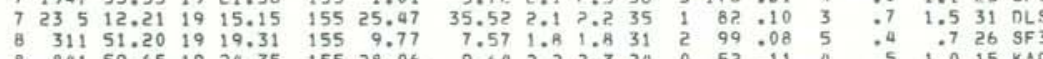

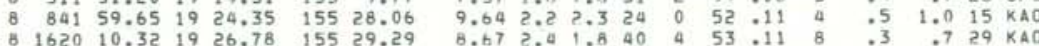

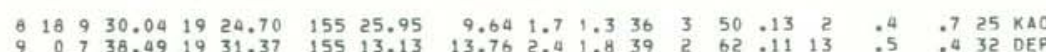

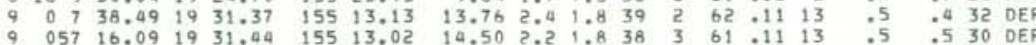

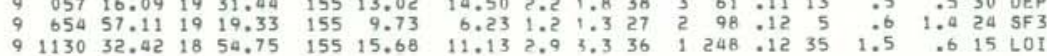

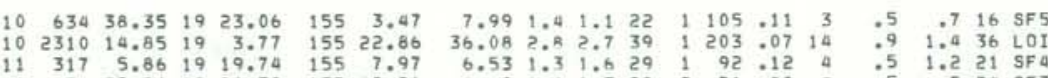

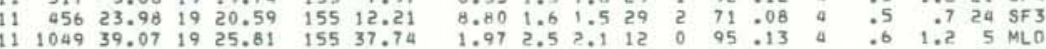

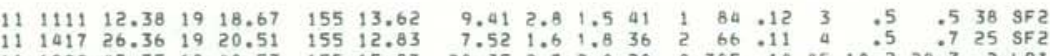

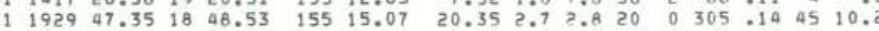

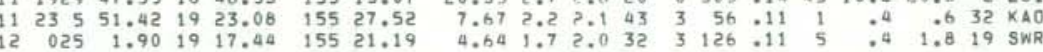

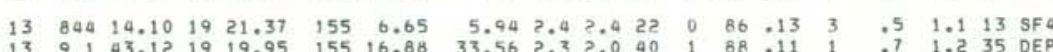

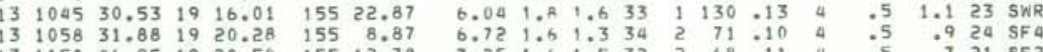

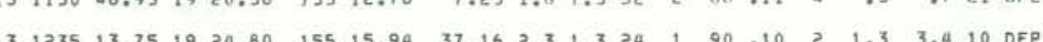

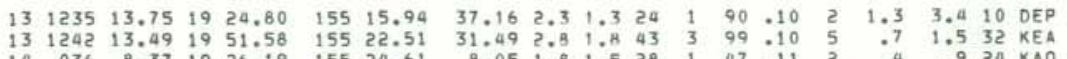

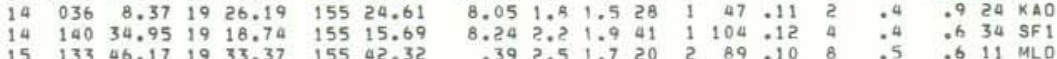

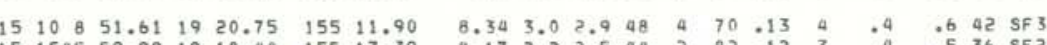

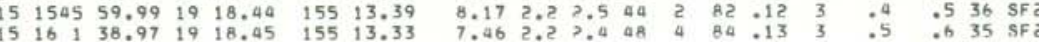




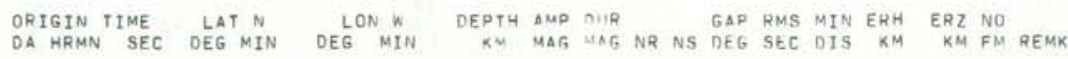

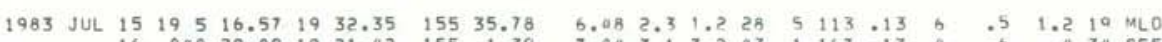

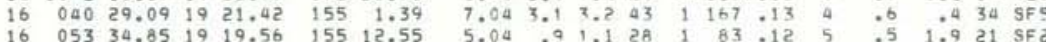

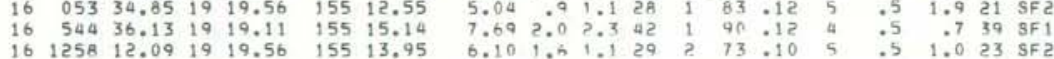

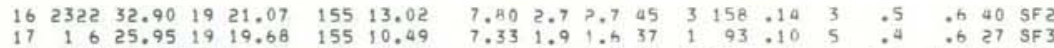

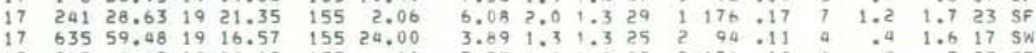

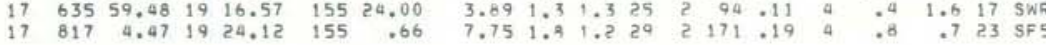
$\begin{array}{llllllllllllllllll}17 & 1041 & 25.18 & 19 & 32.36 & 155 & 36.00 & 7.19 & 2.5 & 1.9 & 34 & 3 & 90 & .11 & 6 & .5 & 1.025 & 2500\end{array}$

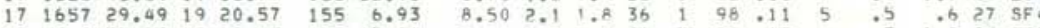

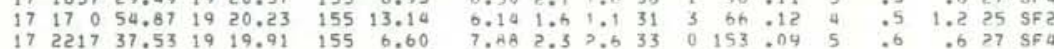
$\begin{array}{lllllllllllllllllll}17 & 2253 & 46.24 & 19 & 21.21 & 155 & 1.77 & 6.51 & 2.0 & .7 & 36 & 2 & 179 & 11 & 8 & .7 & .7 & 29 & \text { SF } \\ 17 & 2257 & 44.65 & 19 & 21.57 & 155 & 14.24 & 25.99 & 1.9 & 1.3 & 37 & 2 & 57 & 010 & 3 & 0.6 & .8 & 33 & \text { DEP }\end{array}$

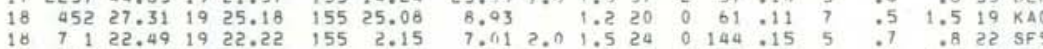

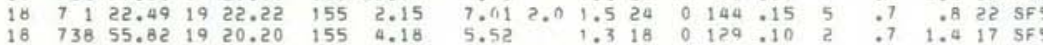

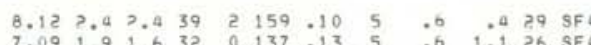

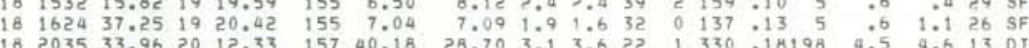

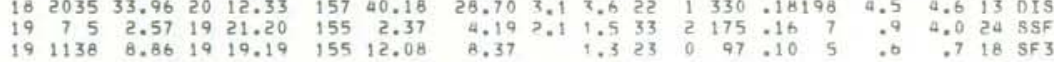

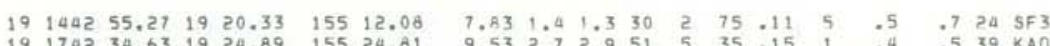

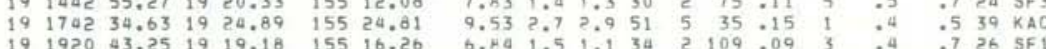

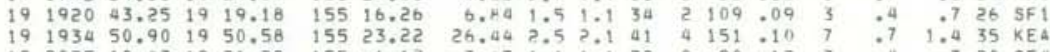

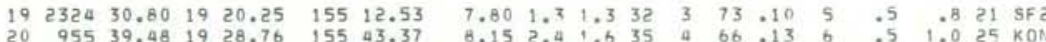

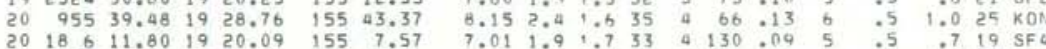

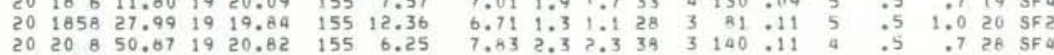
$\begin{array}{lllllllllllllllllll}21 & 655 & 18.47 & 19 & 18.89 & 155 & 11.60 & 5.08 & 1.3 & 1.1 & 31 & 4 & 111 & .10 & 5 & .4 & 1.4 & 17 & \text { SF } 3\end{array}$

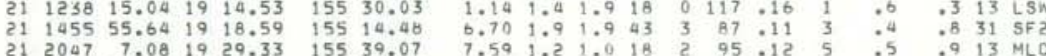

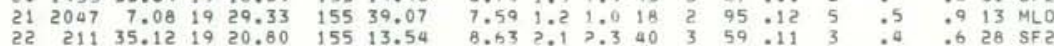
$\begin{array}{lllllllllllllllllll}22 & 629 & 36.74 & 19 & 19.01 & 155 & 13.79 & 7.38 & 1.4 & 1.2 & 30 & 0 & 75 & .10 & 4 & .5 & .9 & 23 & 5 F 2 \\ 22 & 2127 & 27.06 & 19 & 20.11 & 155 & 10.12 & 7.11 & 2.0 & 1.6 & 35 & 2 & 83 & .12 & 4 & .5 & .8 & 26 & 5 F 3 \\ \end{array}$

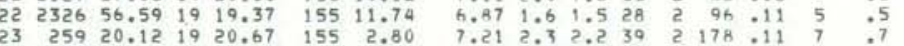

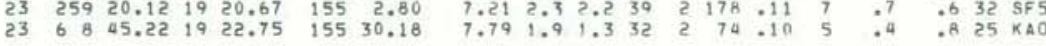

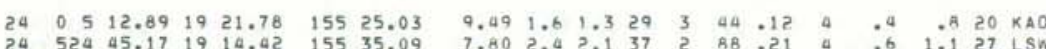

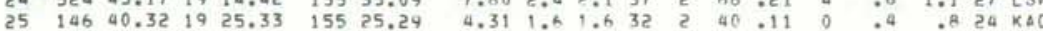

HVO EARTHOUAKE SUMMARY LIST

PAGE 46

ORIGIN TIME L LAT N LON $W$ OEPTH AMP DIR N GAP RMS MIN ERH ERZ NO
OA HRMN SEC DEG MIN DEG MIN KR MAG MAG NR NS DEG SEC OIS KM KM FM REMK

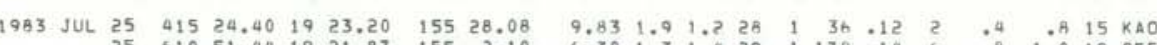

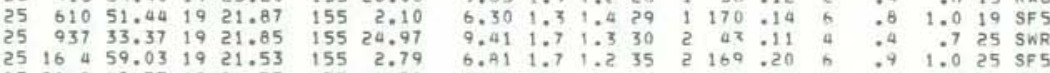

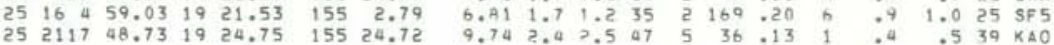
$\begin{array}{lllllllllllllllllllll}26 & 2 & 8 & 47.16 & 19 & 33.59 & 155 & 42.50 & 2.52 & 2.9 & 2.2 & 27 & 1 & 90 & .11 & 8 & .5 & 2.6 & 20 & \text { MLO } \\ 26 & 533 & 28.65 & 19 & 19.83 & 155 & 7.35 & 7.54 & 2.1 & .5 & 33 & 1 & 137 & .09 & 5 & .6 & .7 & 24 & \text { SFu }\end{array}$

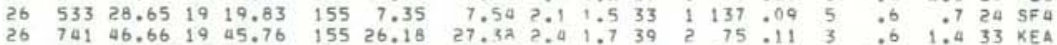

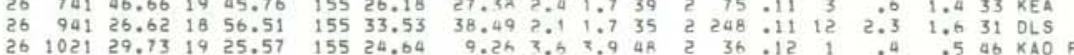

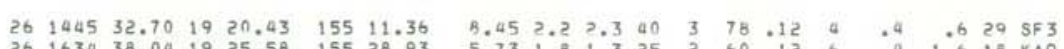

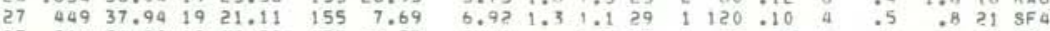

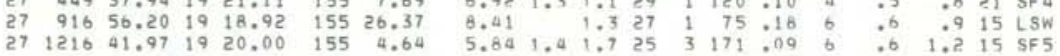

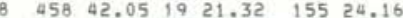

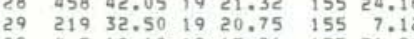

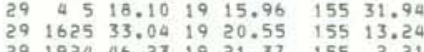

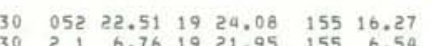
$30 \quad 325 \quad 8.1519 \quad 6.13 \quad 15525.92$ 30
3049
30 $\begin{array}{llllll}30 & 950 & 43.75 & 19 & 21.73 \quad 155 & 15.21\end{array}$ $30 \quad 165221.851921 .90 \quad 155 \quad .65$ 30183434.561922 .77 155 24.98 $31 \quad 34355.23$ 19 $21.11 \quad 155 \quad 8.31$ $31 \quad 40026.06 \quad 19 \quad 19.19 \quad 155 \quad 11.91$

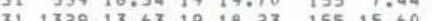

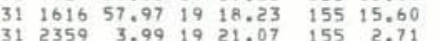

AUG $1 \quad 228 \quad 20.48 \quad 19 \quad 19.68 \quad 155 \quad 10.19$

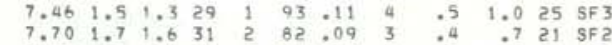

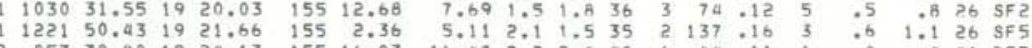

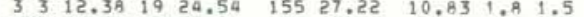
$\begin{array}{llllllll} & \\ 5\end{array}$

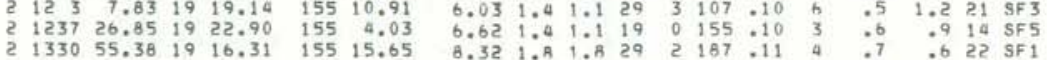

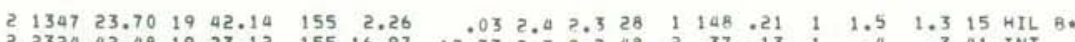

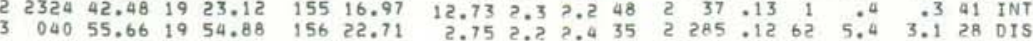


HVO EARTHQUAKE SUMMARY LIST

PAGE 47

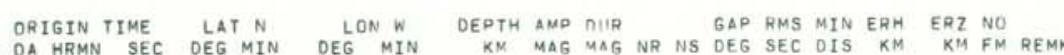

1983 AUG $3 \quad \begin{array}{lllllllllllllllllll}1 & 5 & 38.66 & 19 & 22.12 & 155 & 4.93 & 8.20 & 2.1 & 7.0 & 27 & 1 & 77 & .10 & 3 & .4 & .6 & 20 & 5 F 5\end{array}$

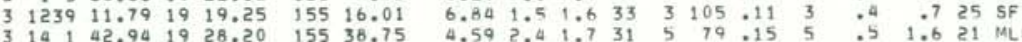

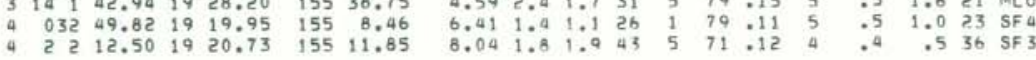

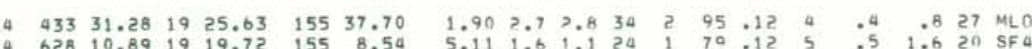

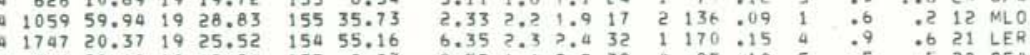

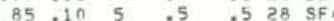

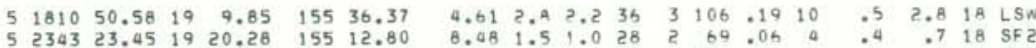

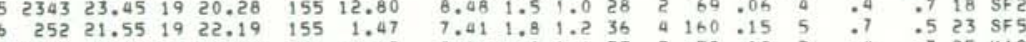

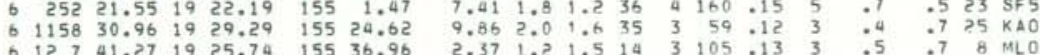

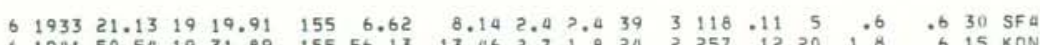

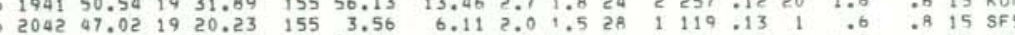

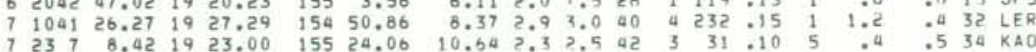

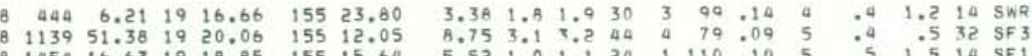

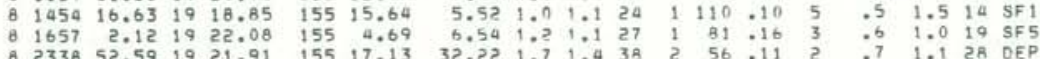

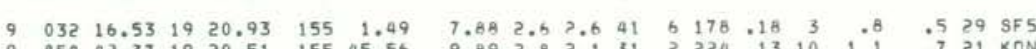

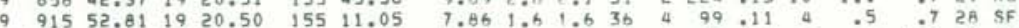

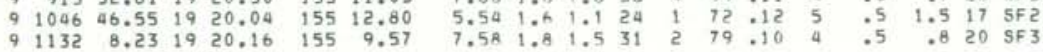

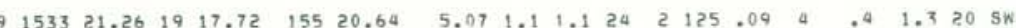

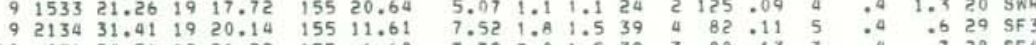

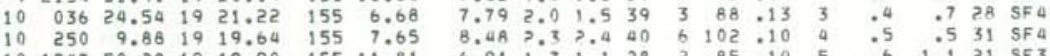

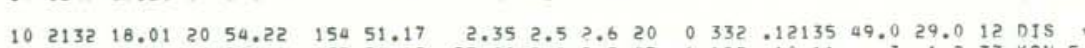

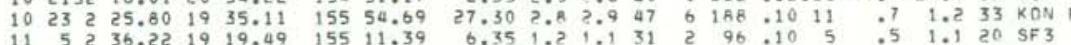

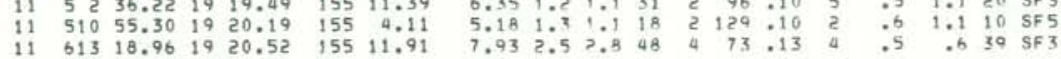

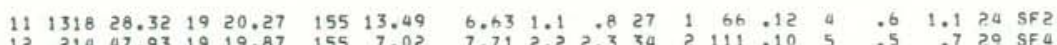

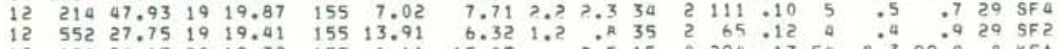

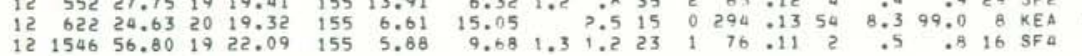

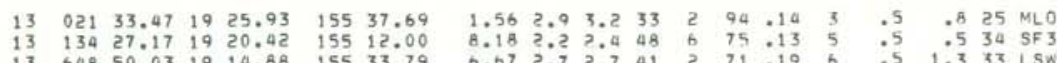

HVO EARTHQUAKE SUMMARY LIST

PAGE 48

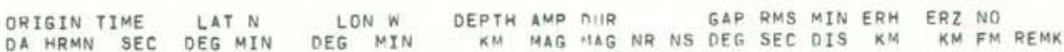

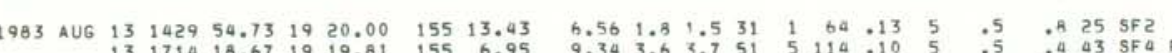

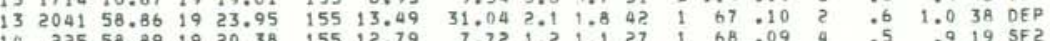

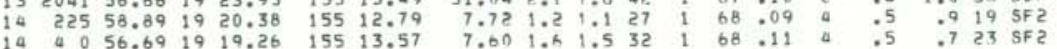

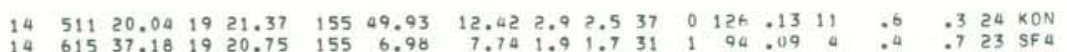

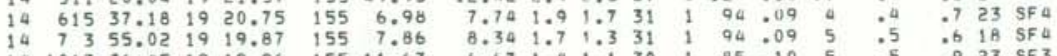

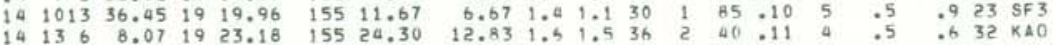

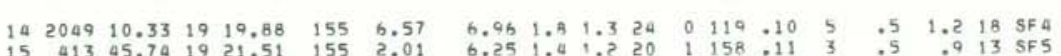

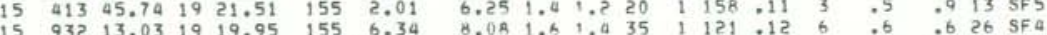

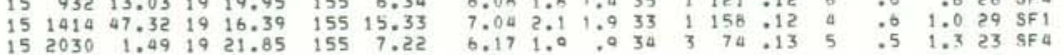

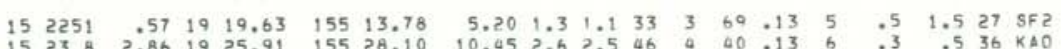

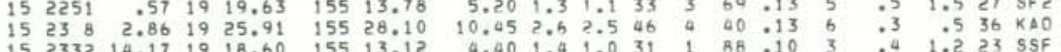

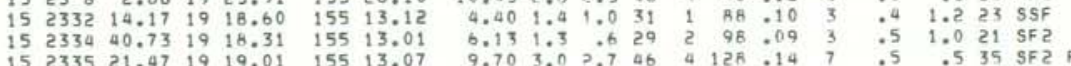

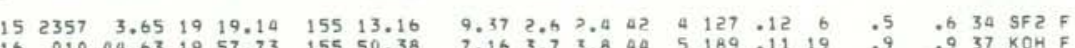

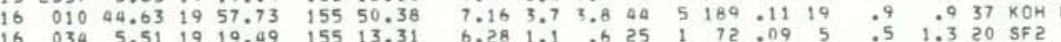

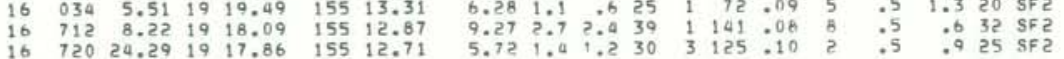

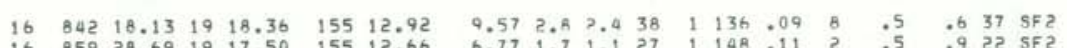

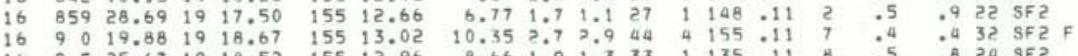

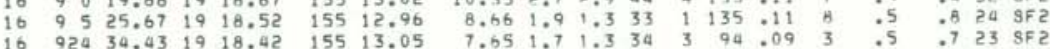

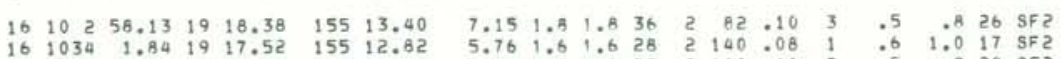

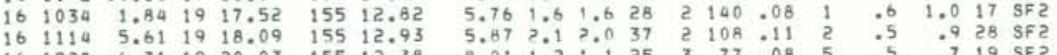
1.301 .14 .1253 1

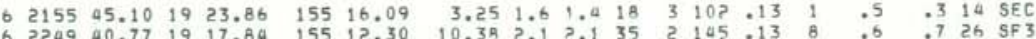

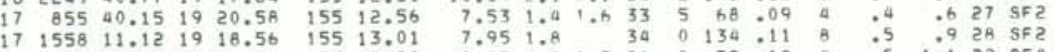

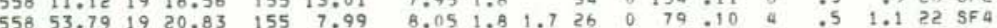

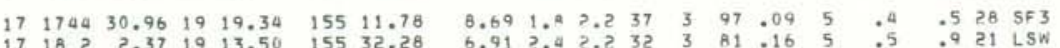

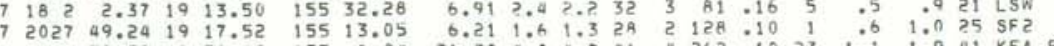

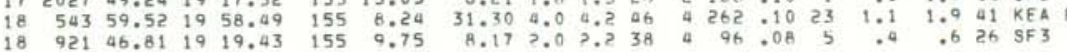

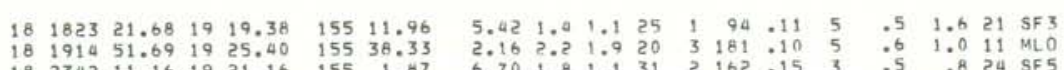




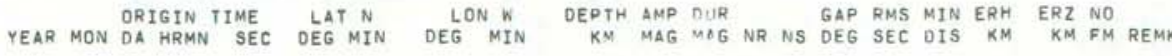

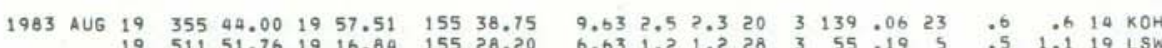

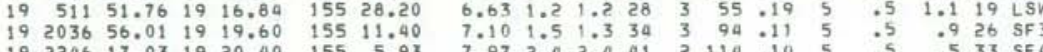

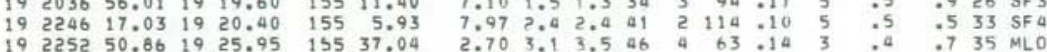

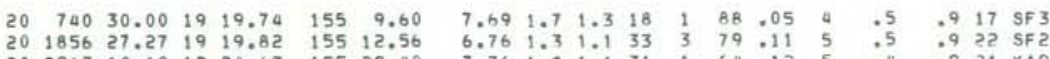

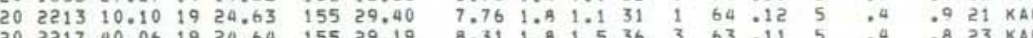

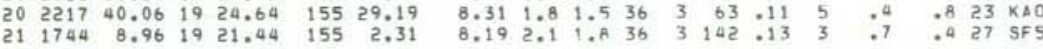

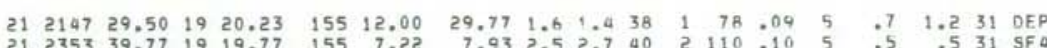

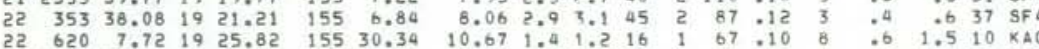

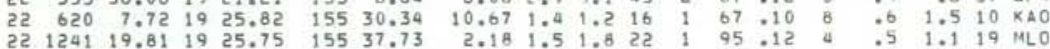

$\begin{array}{lllllllllllllllllll}22 & 1258 & 15.46 & 19 & 20.23 & 155 & 12.77 & 7.69 & 1.5 & 1.1 & 31 & 2 & 70 & .07 & 4 & .5 & .7 & 20 & \text { SF } 2 \\ 22 & 1639 & 35.92 & 19 & 20.31 & 155 & 3.42 & 5.96 & 1.6 & 1.3 & 28 & 1 & 178 & .14 & 7 & .6 & 1.2 & 17 & \text { SF }\end{array}$

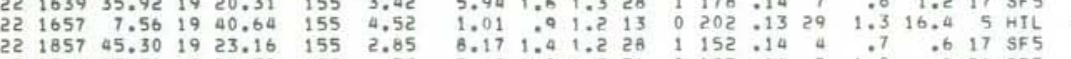

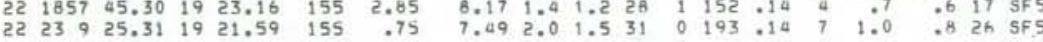
$\begin{array}{lllllllllllllllllll}22 & 2348 & 41.79 & 19 & 24.85 & 155 & 28.80 & 7.56 & 1.6 & 1.3 & 28 & 0 & 46 & 09 & 5 & .0 & 1.1 & 21 & \text { KAO }\end{array}$

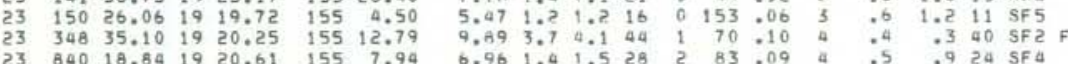
23 34825.101920 .2515512 .79 $\begin{array}{lllllll}23 & 1617 & 44.47 & 19 & 24.22 & 155 & 26.33 \\ 23 & 1713 & 47.24 & 19 & 19.79 & 155 & 10.50\end{array}$ 9495230.011920 .33 155 7.96 $2415742.871924,43 \quad 15525.12$ $\begin{array}{lllllll}24 & 1531 & 29.01 & 19 & 20.00 & 155 & 11.83\end{array}$ $242238 \quad 51.031921 .83 \quad 15524.99$

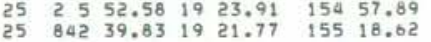

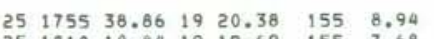
$25 \quad 1810 \quad 10.84 \quad 19 \quad 19.69 \quad 155 \quad 7.68$ $\begin{array}{lllllll}25 & 1833 & 1.11 & 19 & 19.98 & 155 & 8.10 \\ 25 & 1835 & 21.61 & 19 & 19.52 & 155 & 7.46\end{array}$ $\begin{array}{lllllll}26 & 351 & 12.53 & 19 & 25.28 & 155 & 38.21 \\ 26 & 531 & 18.74 & 19 & 15.95 & 155 & 19.63\end{array}$ $26 \quad 623 \quad 52.10 \quad 19 \quad 25.29 \quad 155 \quad 37.45$ $\begin{array}{lrrrrrr}26 & 722 & 58.90 & 19 & 25.34 & 155 & 29.26 \\ 26 & 1813 & 42.53 & 19 & 23.19 & 155 & 2.28\end{array}$ $\begin{array}{lrlllll}26 & 2357 & 10.43 & 19 & 21.95 & 155 & 25.72 \\ 27 & 111 & 38.89 & 19 & 19.58 & 155 & 11.89 \\ 27 & 47 & 34.42 & 19 & 20.11 & 155 & 8.69\end{array}$

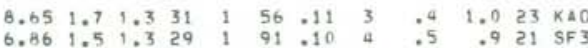

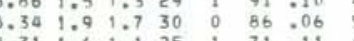

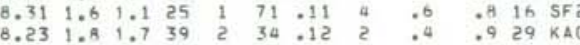

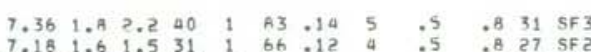

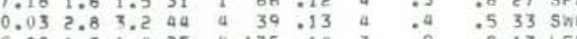

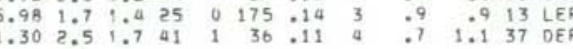

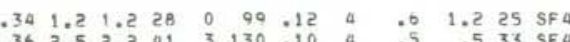

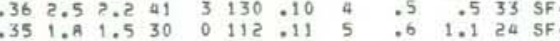

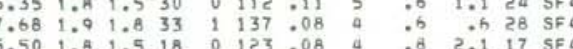

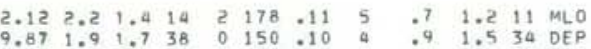

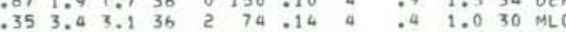

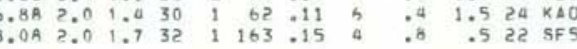

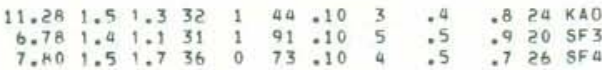
YEAR MON DA HRMN SEC OEG MIN DEG MIN OEPTH AMP OIR MA GA GAP RMS MIN ERH ERZ NO

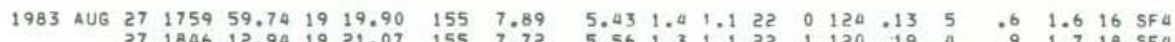

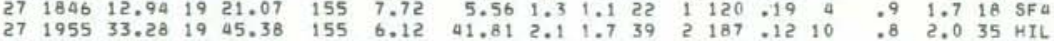

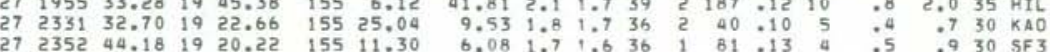

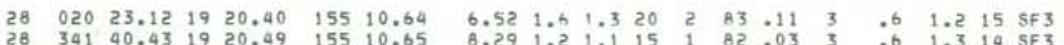

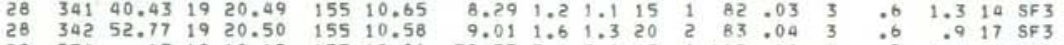

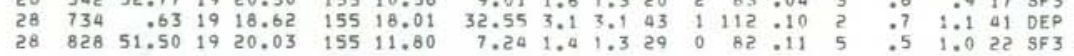
\begin{tabular}{lllllllllllllllllll}
28 & 1546 & 17.34 & 19 & 22.66 & 155 & 1.12 & 8.23 & 2.0 & 1.2 & 30 & 1 & 178 & .15 & 6 & 1.1 & .7 & 19 & SF 5 \\
\hline
\end{tabular}

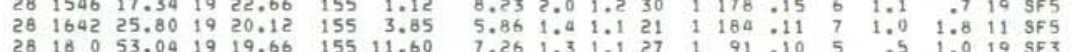

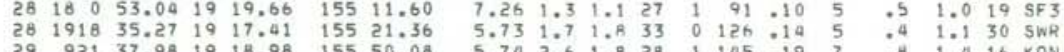

$\begin{array}{lllllllllllllllllllll}29 & 1212 & 12.81 & 19 & 19.50 & 155 & 11.80 & 5.40 & 1.4 & 1.1 & 27 & 1 & 93 & .13 & 5 & .5 & 1.3 & 23 & 5 F 3\end{array}$

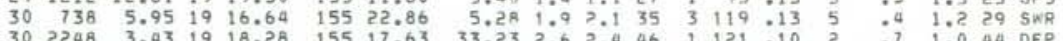

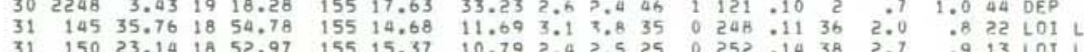
$\begin{array}{llllllllllllllllllll}31 & 2 & 2 & 12.80 & 18 & 54.22 & 155 & 15.70 & 13.10 & 2.6 & 2.7 & 23 & 0 & 257 & .13 & 35 & 2.9 & 1.2 & 10 & \text { LOI }\end{array}$

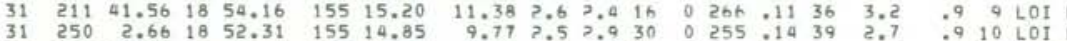

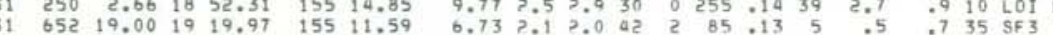

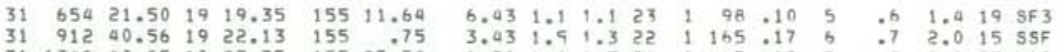

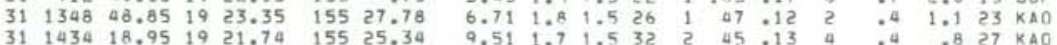

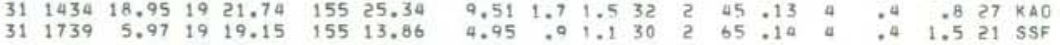

$\begin{array}{lllllllllllllllllll}\text { SEP } 1 & 110 & 34.79 & 19 & 19.57 & 155 & 12.16 & 5.41 & 1.6 & 1.5 & 40 & 2 & 87 & .12 & 5 & .4 & 1.0 & 31 & \text { SF } 3\end{array}$

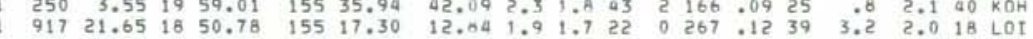

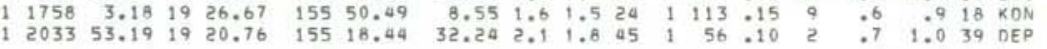

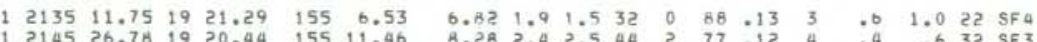

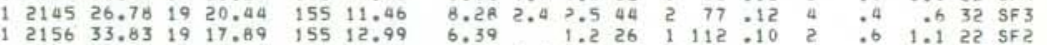

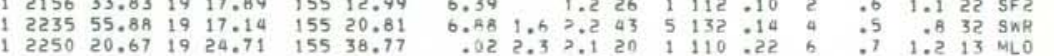

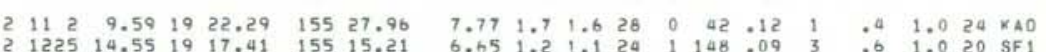

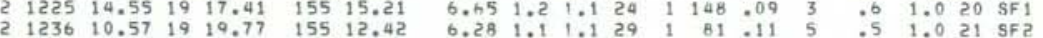

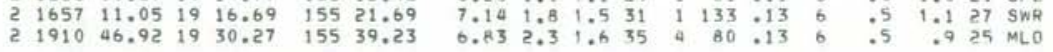

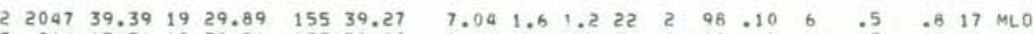

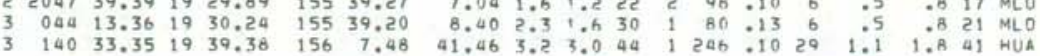


HVO EARTHQUAKE SUMMARY LIST

PAGE 5

ORIGIN TIME
DA HRMN SAT

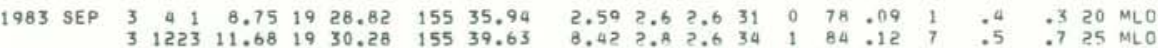

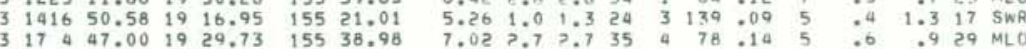

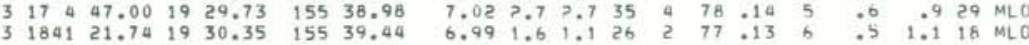

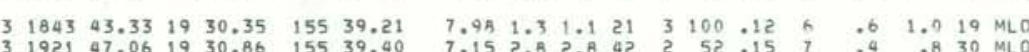

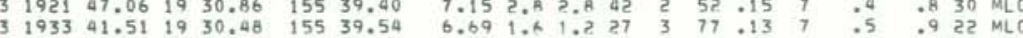

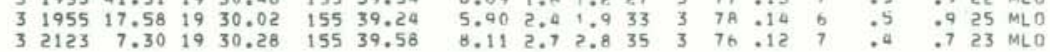

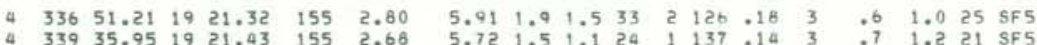

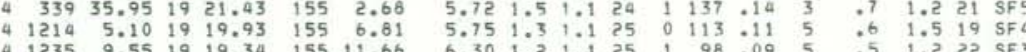

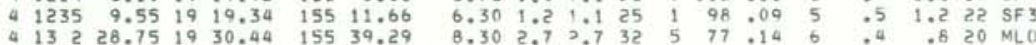

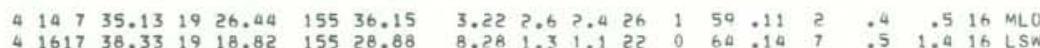

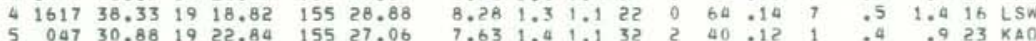

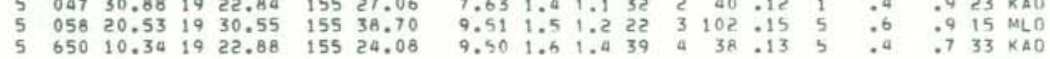

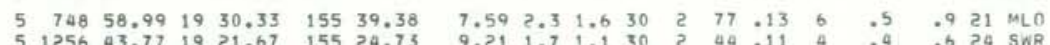

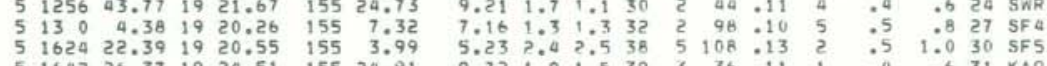

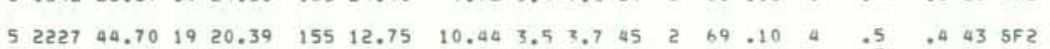

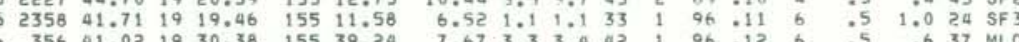

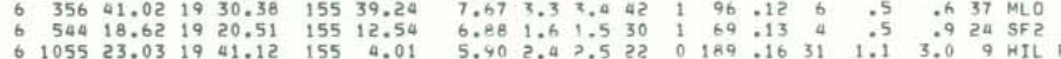

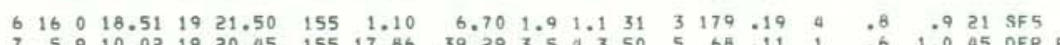

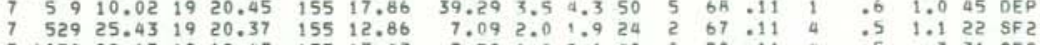

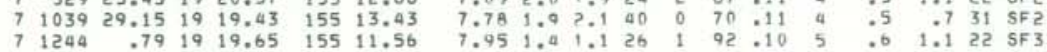

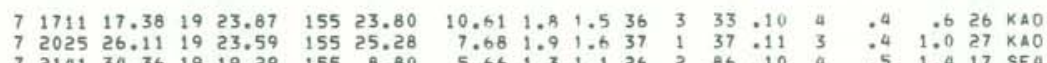

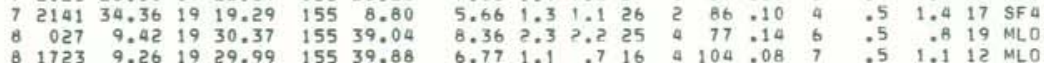

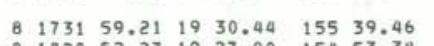

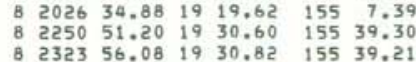

$82332 \quad 17.81 \quad 1929.96 \quad 15539.8$

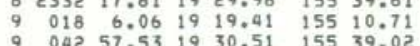

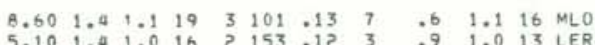

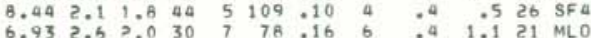

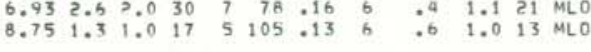
$\begin{array}{lllllllllllll}5.90 & 1.9 & 1.2 & 18 & 4 & 103 & 07 & 7 & .5 & 1.3 & 13 & M L O \\ 8.11 & 1.8 & 1.1 & 26 & 0 & 100 & : 09 & 5 & .6 & 1.0 & 26 & \text { SF } & \end{array}$
HVO EARTHDUAKE SUMMARY LIST

PAGE 52

ORIGIN TIME LAT N LON W DEPTH AMP MUR GAP RMS MIN ERH ERT NO YEAR MON DA HPMN 1983 SEP 9

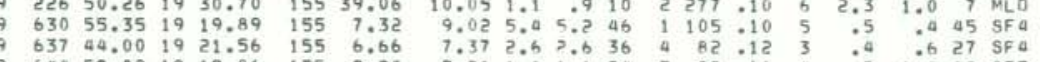

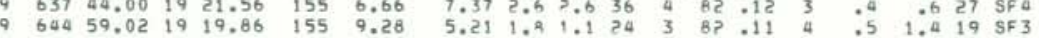
$\begin{array}{llllllllllllllllllll}9 & 726 & 20.73 & 19 & 18.00 & 155 & 6.87 & 3.17 & 1.4 & 1.0 & 15 & 1 & 176 & .09 & 2 & 1.2 & .8 & 12 & \text { SSF }\end{array}$ 75737,76
9

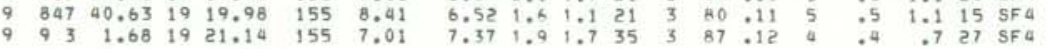
$\begin{array}{lllllll}9 & 914 & 29.63 & 19 & 19.77 & 155 & 6.03\end{array}$

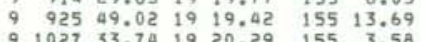

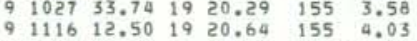
$91143 \quad 27.31 \quad 1920.52 \quad 155 \quad 11,45$ $\begin{array}{llllllllllll}3.96 & 2.0 & 1.6 & 34 & 1 & 131 & .17 & 5 & .6 & 2.1 & 20 & \text { SSF }\end{array}$

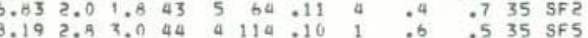

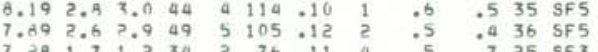

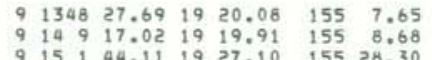

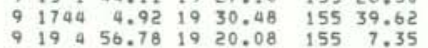
$92028 \quad 12.23 \quad 19 \quad 10.03 \quad 155 \quad 37.86$ $9232 \begin{array}{lllll}93.70 & 19 & 21.36 & 155 & 6.45\end{array}$ $\begin{array}{lllllll}10 & 229 & 19.53 & 19 & 20.69 & 155 & 12.94 \\ 10 & 549 & 44.90 & 19 & 23.02 & 155 & 2.44\end{array}$

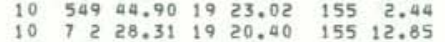
$\begin{array}{lllllll}10 & 810 & 49.07 & 19 & 17.93 & 155 & 13.28 \\ 10 & 840 & 25.93 & 19 & 20.83 & 155 & 3.32\end{array}$ 10.1392515 19 $10.03 \quad 155 \quad 33.32$

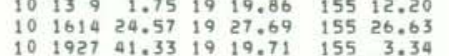

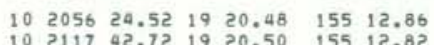

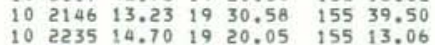

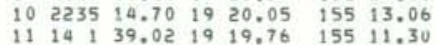

$\begin{array}{lllllll}11 & 1513 & 14.77 & 19 & 31.12 & 155 & 39.28 \\ 11 & 1931 & 21.62 & 19 & 20.57 & 155 & 12.79\end{array}$

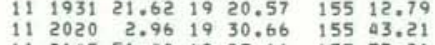

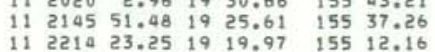
$\begin{array}{llllllll}11 & 23 & 2 & 28.57 & 19 & 30.17 & 155 & 39.23\end{array}$

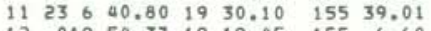

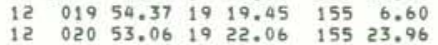
$\begin{array}{lllllll}12 & 332 & 15.30 & 19 & 19.37 & 155 & 13.05 \\ 12 & 351 & 59.17 & 19 & 30.23 & 155 & 38.81\end{array}$

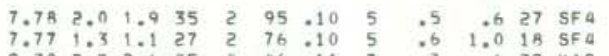

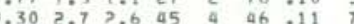

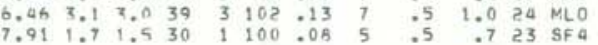
$\begin{array}{lllllllllllll}8.52 & 2.6 & 2.2 & 33 & 3 & 115 & .13 & 9 & .4 & .8 & 21 & \text { LSW }\end{array}$

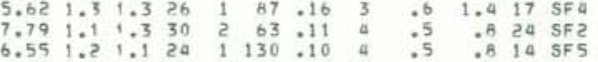
$\begin{array}{rlllllllllllll}6.55 & .2 & 1.1 & 2 a & 1 & 130 & 10 & 4 & .5 & .8 & 14 & 5 F 5 \\ 6.28 & 1.6 & 1.5 & 36 & 2 & 67 & 14 & 4 & : 5 & .8 & 29 & 5 F 2\end{array}$ $\begin{array}{lllllllllllll}6.71 & 1.4 & 1.3 & 30 & 3 & 95 & .09 & 2 & .5 & .8 & 19 & \text { SF } 2 \\ 0.48 & .6 & 1 & 29 & 2 & 98 & 09 & 11 & 2 & .5 & : 9 & 15 & \text { SFS }\end{array}$

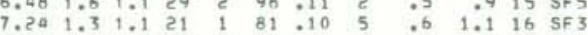

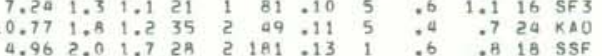
$\begin{array}{llllllllllll}7.83 & 1.6 & 1.6 & 35 & 2 & 66 & .11 & 4 & .5 & .6 & 23 & \mathrm{sF}\end{array}$

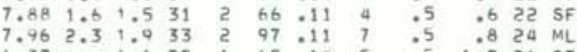
$\begin{array}{llllllllllll}6.33 & & 1.1 & 29 & 1 & 69 & 114 & 5 & 0.5 & 1.2 & 26 & 5 F 2 \\ 5.15 & 1.7 & 1.5 & 33 & 1 & 40 & -11 & 6 & : 4 & 1.3 & 26 & 5 F 3\end{array}$ $\begin{array}{llllllllllll}6.55 & 2.9 & 2 . A & 38 & 6 & 80 & .17 & 7 & .4 & .9 & 21 & M L 0 \\ 7.00 & 1.6 & 1: 1 & 26 & 1 & 66 & 011 & 4 & .5 & : 9 & 19 & \text { SF }\end{array}$

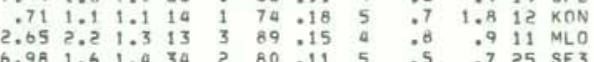

$\begin{array}{llllllllllllll}8.70 & 1.3 & .0 & 19 & 3 & 100 & .16 & 6 & .7 & 1.1 & 12 & \text { MLO } & 0\end{array}$

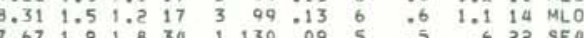

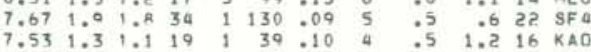

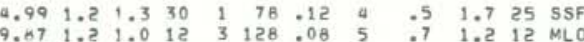


HVO EARTHQUAKE SUMMARY LIST

PAGE 53

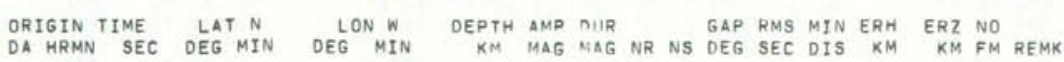
YEAR MON DA HRMN SEC DEG MIN DEG MIN KM MAGG NAG NR NS DEG SEC DIS KM KM FM REM

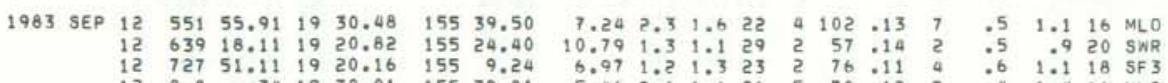

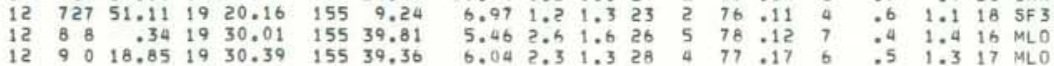

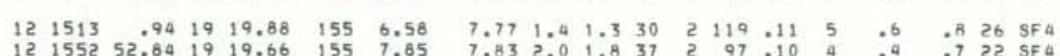

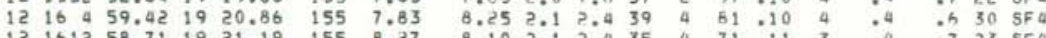

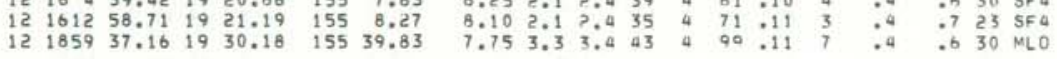

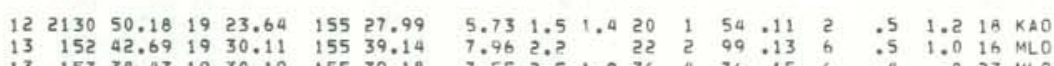

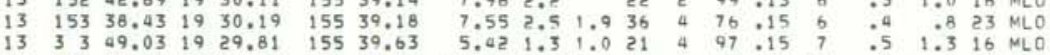

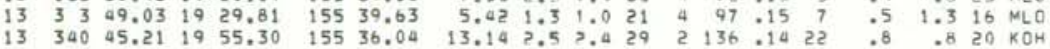

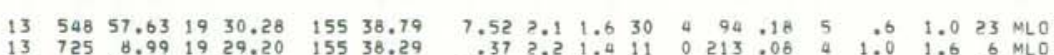

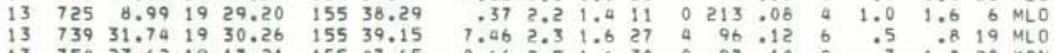

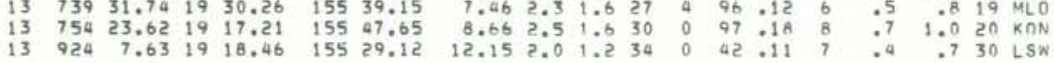

$13 \quad 1215 \quad 42.22 \quad 19 \quad 19.85 \quad 155 \quad 9.99$

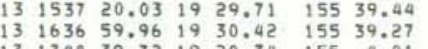

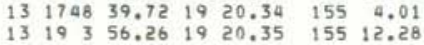
$\begin{array}{lllllll}13 & 1939 & 57.53 & 19 & 21.25 & 155 & 3.14 \\ 13 & 1943 & 43.11 & 19 & 21.09 & 155 & 3.21\end{array}$

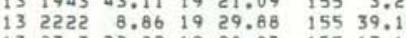

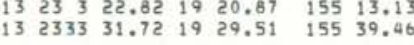

$14 \quad 04925.86 \quad 19 \quad 30.34 \quad 155 \quad 38.86$ $14 \quad 216 \quad 51.74 \quad 1929.60 \quad 15539.25$

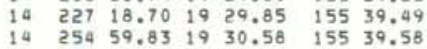

$\begin{array}{lrrrrrr}14 & 515 & 59.32 & 19 & 17.96 & 155 & 13.29 \\ 14 & 523 & 2.39 & 19 & 21.13 & 155 & 1.35\end{array}$ 1455718.28 19 46.17 155 34.48

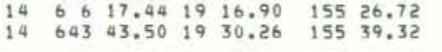

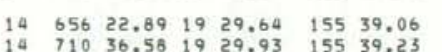
$14 \quad 720 \quad 23.56$ 19 $29.91 \quad 15559.19$

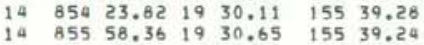
$\begin{array}{lllllll}14 & 921 & 18.93 & 19 & 30.23 & 155 & 39.17 \\ 14 & 955 & 24.11 & 19 & 30.12 & 155 & 39.11\end{array}$

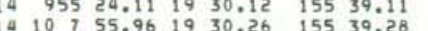

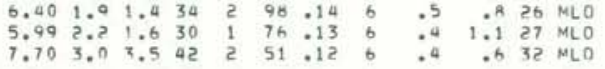
$\begin{array}{llllllllllllll}6.57 & 2.3 & 1.6 & 27 & 3 & 80 & .13 & 6 & .5 & 1.0 & 23 & \text { MLO } \\ 5.31 & 2.3 & 2.1 & 38 & 5 & 76 & .13 & 6 & .5 & 1.3 & 32 & \text { MLO } \\ 6.12 & 2.4 & .5 & 36 & 3 & 80 & .13 & 6 & .5 & 1.1 & 30 & & & \end{array}$
HVO EARTHQUAKE SUMMARY LIST

PAGE 54

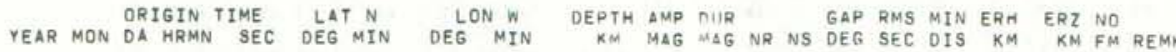
1983 SEP $141016 \quad 41.321929 .64 \quad 15539.13$ $14 \quad 1048 \quad 32.26 \quad 19 \quad 18.17 \quad 15513.28$ 4 1258 17.08 19 $31.02 \quad 155 \quad 39.51$

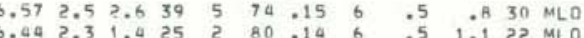

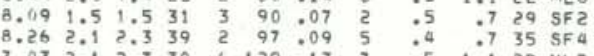

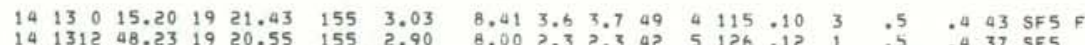

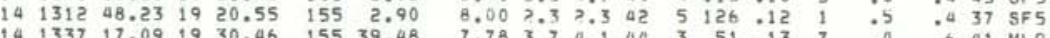

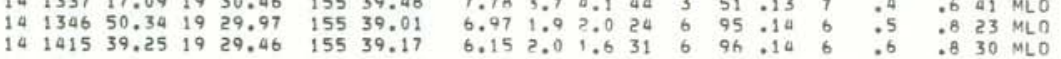

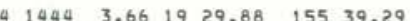
$\begin{array}{llllllllllllll}6.45 & 2.3 & 2.0 & 39 & 6 & 75 & .14 & 6 & .4 & .7 & 36 & \text { MLO }\end{array}$

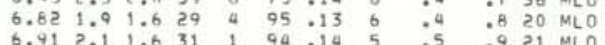
$\begin{array}{lllllllllllll}6.91 & 2.1 & 1.6 & 31 & 1 & 94 & 14 & 5 & .5 & .9 & 21 & M L 0 \\ 4.94 & 2.1 & 1.5 & 23 & 2 & 96 & -14 & 6 & : 5 & 1.6 & 18 & M L 0\end{array}$ $1015912.111929 .82 \quad 15538.96$ $1417248.76 \quad 1929.72 \quad 15539.25$ $\begin{array}{lllllll}14 & 2014 & 35.17 & 19 & 24.69 & 155 & 34.02 \\ 14 & 2239 & 31.17 & 19 & 20.30 & 155 & 12.00\end{array}$ $14.235635 .591920 .30 \quad 155 \quad 12.00$

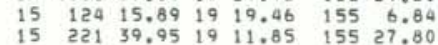
$\begin{array}{lllllll}15 & 338 & 30.46 & 19 & 30.31 & 155 & 38.97\end{array}$ $15 \quad 6952.9519 \quad 30.12 \quad 15538.61$

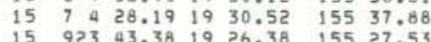

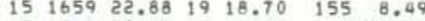
$15181920.171911 .92 \quad 155 \quad 28.36$ $15185828.571930 .46 \quad 155$ 29.85

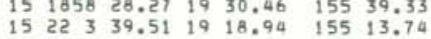

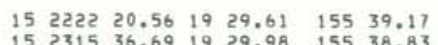

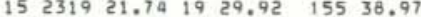

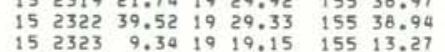
$\begin{array}{lllllll}16 & 138 & 4.88 & 19 & 29.78 & 155 & 38.98\end{array}$

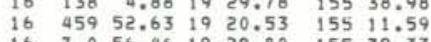
$16 \quad 93211.461930 .80 \quad 15539.33$ $1693721.05 \quad 1929.50 \quad 15539.01$

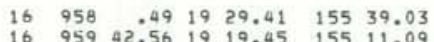
$16 \quad 1024 \quad 3.8819 \quad 29.78 \quad 155 \quad 39.16$

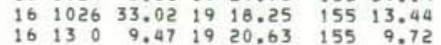
$\begin{array}{lllllll}16 & 159 & 53.21 & 19 & 30.08 & 155 & 39.06 \\ 16 & 1512 & 22.82 & 19 & 29.34 & 155 & 38.92\end{array}$

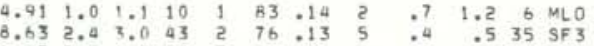

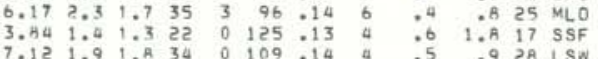
$\begin{array}{lllllllllllll}6.13 & 2.3 & 3 & 20 & 26 & 4 & 94 & 19 & 6 & .5 & 1.4 & 21 & \text { MLO } \\ 5.16 & 2.0 & 1.5 & 35 & 4 & 137 & 0.20 & 5 & : 6 & 1: 6 & 22 & 5 F 5\end{array}$

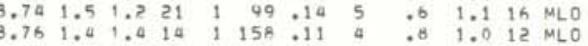
$\begin{array}{llllllllllll}6.63 & 1.7 & 1.3 & 38 & 3 & 92 & .10 & 3 & .5 & .9 & 2 h & \text { SF } 4\end{array}$

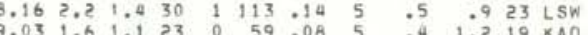

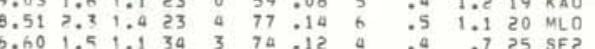

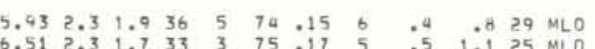
$\begin{array}{llllllllllll}6.93 & 1.9 & 1.2 & 19 & 1 & 75 & .12 & 5 & .5 & 1.0 & 14 & \text { MLO }\end{array}$ $\begin{array}{llllllllllll}6.02 & 2.4 & 1.4 & 32 & 3 & 92 & .13 & 5 & .5 & .9 & 25 & \text { MLO } \\ 7.30 & 1.9 & 1.5 & 31 & 1 & 76 & .11 & 4 & .5 & .6 & 23 & \text { SF }\end{array}$ $6.62 \begin{array}{lllllllllll}6.2 & 1.5 & 34 & 3 & 78 & 14 & 5 & .4 & .9 & 24 & M L O\end{array}$

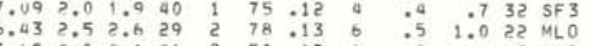
$\begin{array}{lllllllllll}0.63 & 0.1 & 46 & 2 & 50 & 0.12 & 6 & .4 & .6 & 43 & \text { MLO }\end{array}$

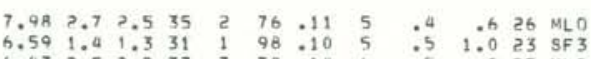

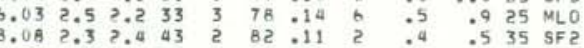

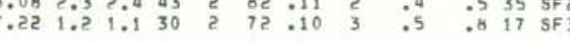

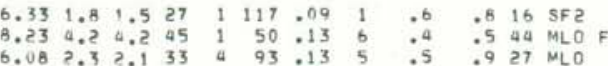


HVO EARTHQUAKE SUMMARY LIST

PAGE SS

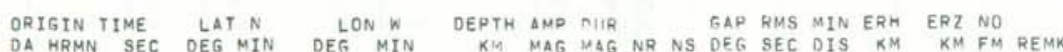

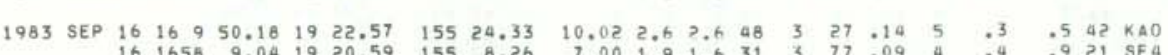

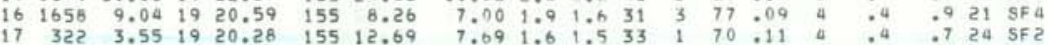

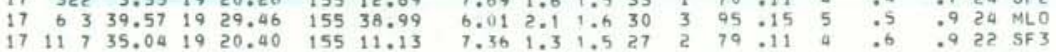

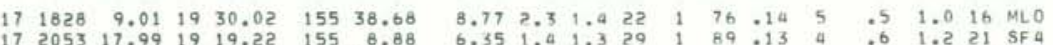

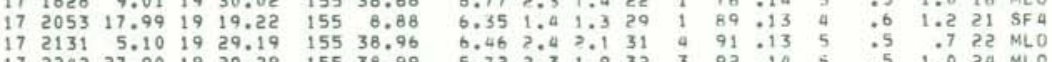

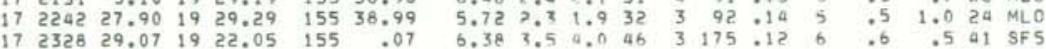

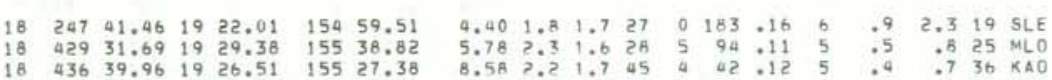

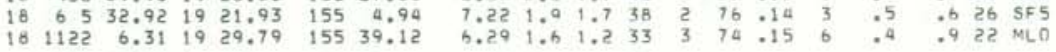

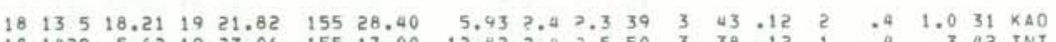

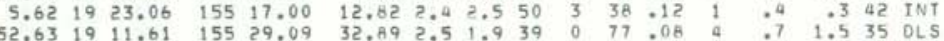

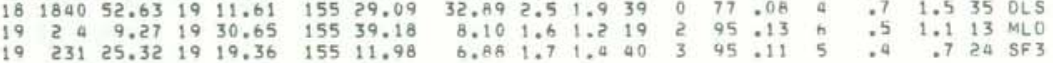

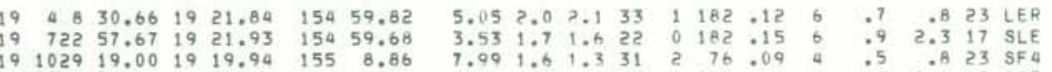

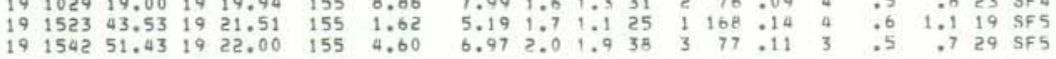

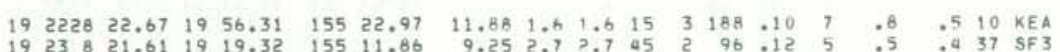

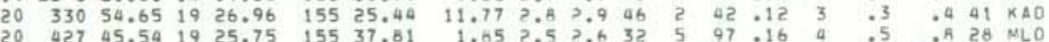

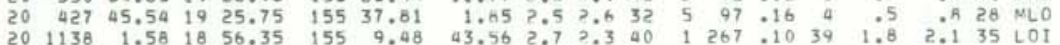

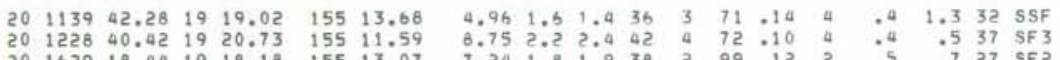

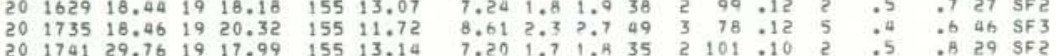

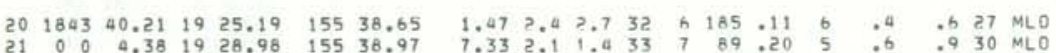

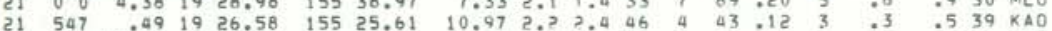

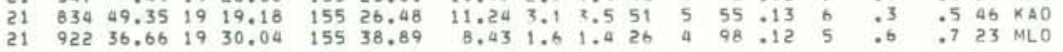

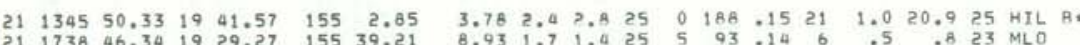

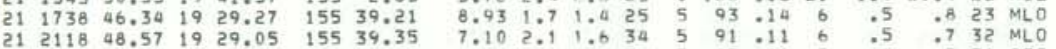

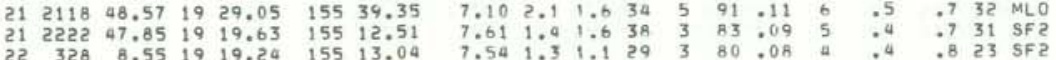

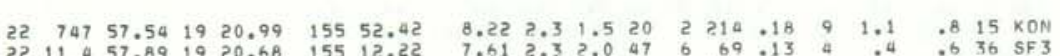

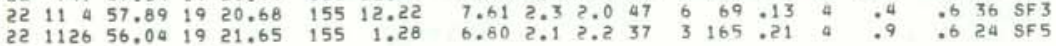

hVO EARTHQUAKE SUMMARY LIST

PAGE 56

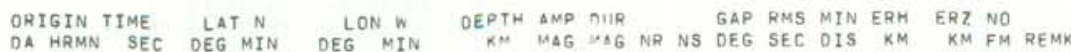

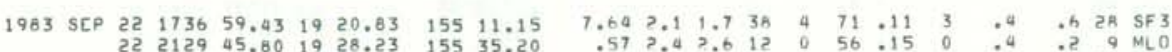

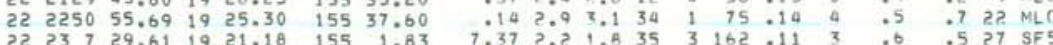

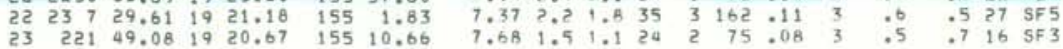

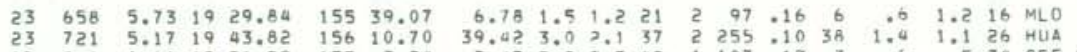

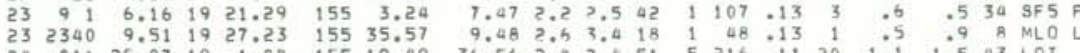

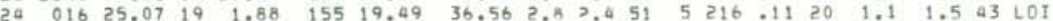

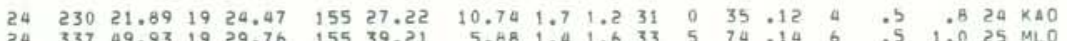

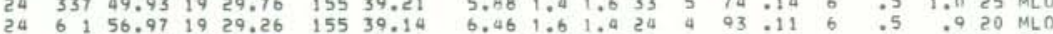

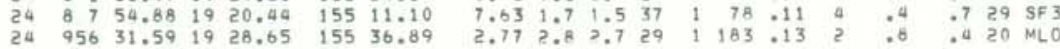
$\begin{array}{lllllllllllllllllll}24 & 1519 & 16.54 & 19 & 27.99 & 155 & .66 & 43.83 & 2.5 & 2.5 & 46 & 3 & 105 & .09 & 6 & .9 & 1.3 & 40 & \text { DEP }\end{array}$

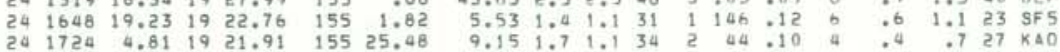

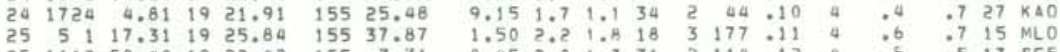

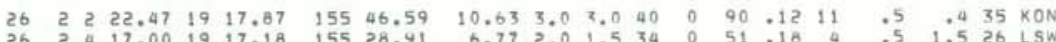

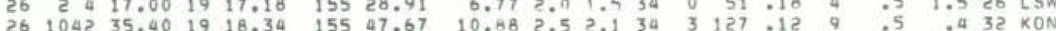

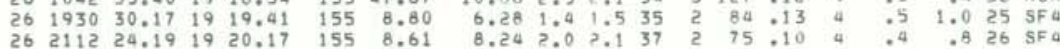

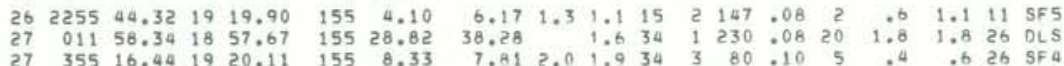

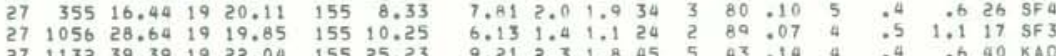

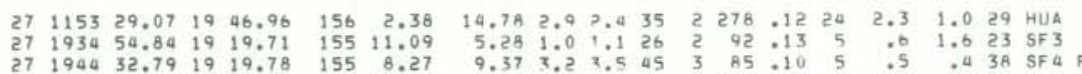

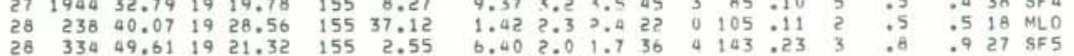
$\begin{array}{lllllllllllllllllllll}28 & 457 & 33.16 & 19 & 20.07 & 155 & 10.49 & 7.45 & 1.3 & 1.1 & 24 & 2 & 85 & .09 & 4 & .6 & 1.0 & 18 & \text { SF } 3\end{array}$

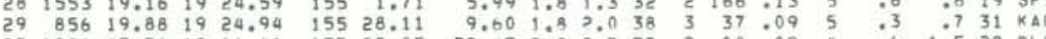

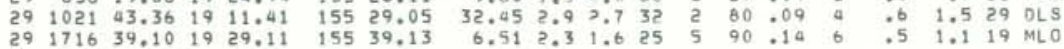

$\begin{array}{llllllllllllllllllll}30 & 226 & 28.58 & 19 & 20.84 & 155 & 12.94 & 7.65 & 2.1 & 1.9 & 40 & 4 & 62 & 12 & 3 & .4 & .6 & 36 & 5 F 2 \\ 30 & 247 & 13.24 & 19 & 21.29 & 155 & 7.03 & 6.74 & 1.5 & 1.1 & 29 & 2 & 84 & 0.12 & 3 & : 6 & .09 & 21 & 5 F 4\end{array}$

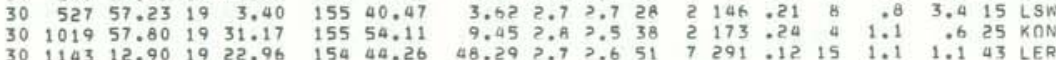

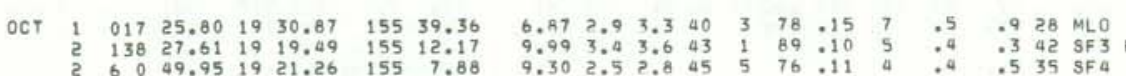


ORIGIN TIME LAT N
YEAR MON DA HRMN SEC DEG MIN DE W MIN OEPTH AMP DIR NR GAP RMS MIN ERH ERZ ND
KM MAG MAG NR NS DEG SEC OIS KM KM FM REMK

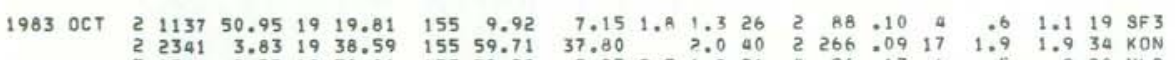

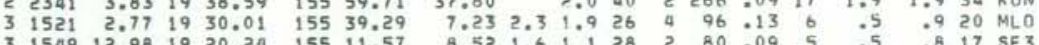

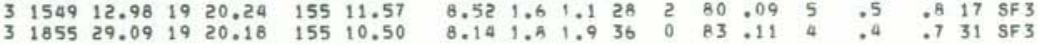

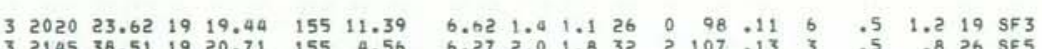

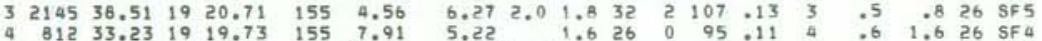

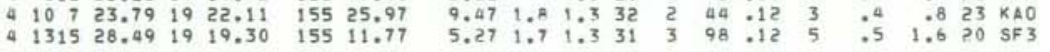
$\begin{array}{lllllllllllllllllll}4 & 1428 & 16.53 & 19 & 19.44 & 155 & 8.50 & 5.16 & 1.7 & 1.1 & 27 & 2 & 82 & .10 & 4 & .5 & 1.5 & 23 & 5 F 4 \\ 4 F 4\end{array}$

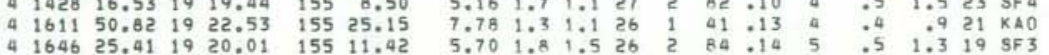

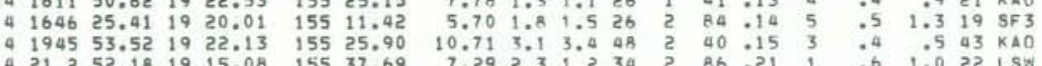

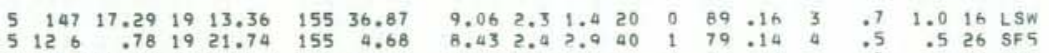

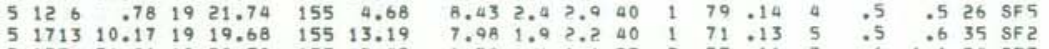

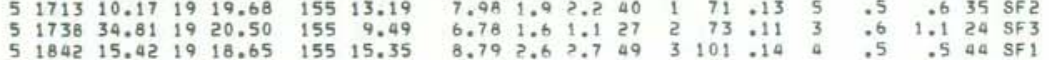

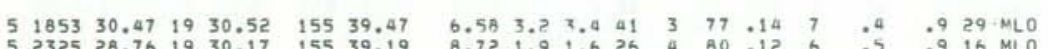
6353
6
6

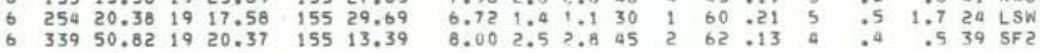

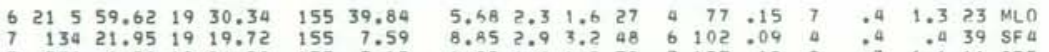

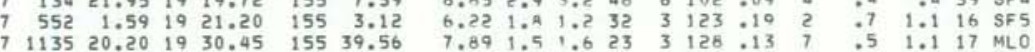

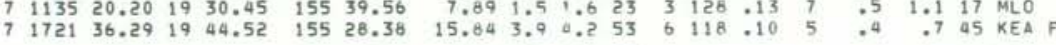

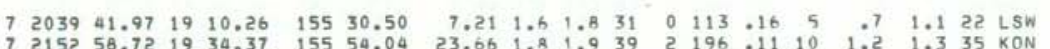

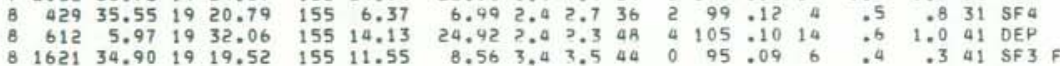

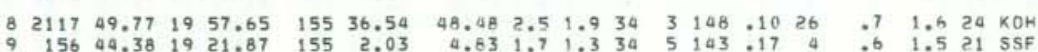

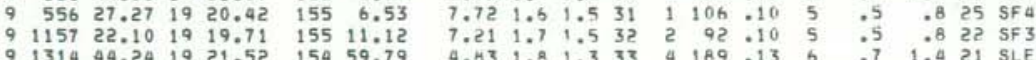

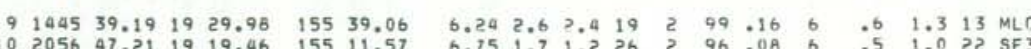

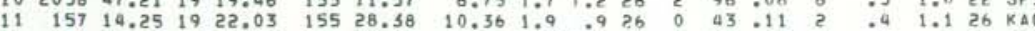

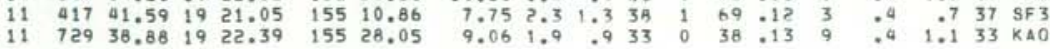

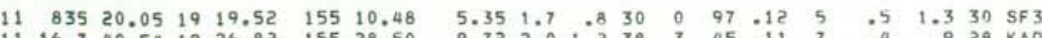

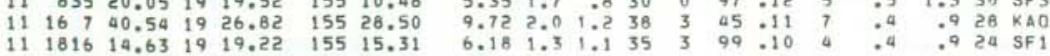

ORIGIN TIME LAT N LON W DEPTH AMP NIIR GAP RMS MIN ERH ERZ NO YEAR MON DA HRMN

$\begin{array}{llllllllllllllllllllllll}1983 & \text { OCT } 11 & 19 & 1 & 39.54 & 20 & 9.96 & 155 & 52.98 & 25.94 & 1.9 & 31 & 1 & 288 & 12 & 12 & 2.8 & 1.6 & 22 & \mathrm{KOH}\end{array}$

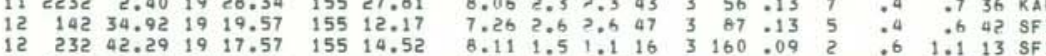

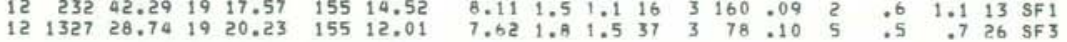

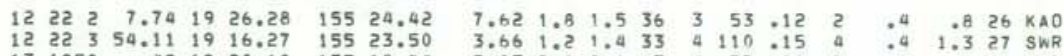

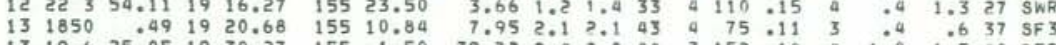

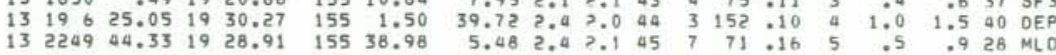
$\begin{array}{llllllllllllllllll}14 & 426 & 44.19 & 19 & 30.84 & 155 & 39.49 & 7.42 & 1.5 & 1.4 & 20 & 5 & 79 & .13 & 7 & .5 & 1.2 & \text { 1h MLO }\end{array}$

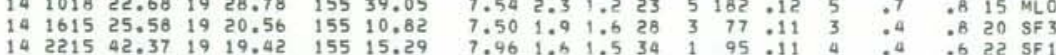

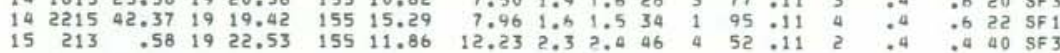

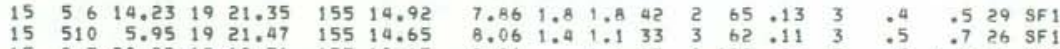

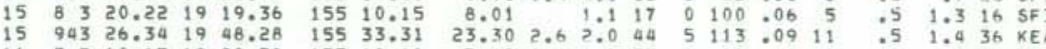

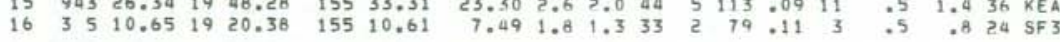

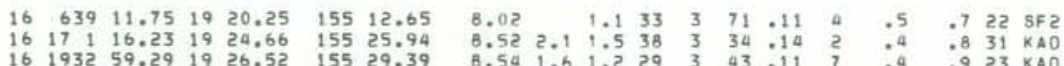

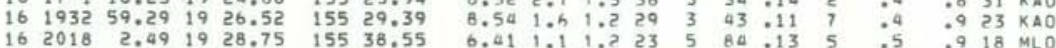

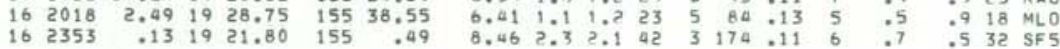

$\begin{array}{llllllllllllllllllll}17 & 124 & 9.74 & 19 & 23.15 & 155 & 26.43 & 8.45 & 2.1 & 1.7 & 37 & 2 & 31 & 12 & 2 & .4 & .7 & 31 & \text { KAO } \\ 17 & 957 & 30.59 & 19 & 20.21 & 155 & 12.42 & 7.74 & 2.0 & 1.3 & 31 & 2 & 74 & : 11 & 5 & : 5 & .7 & 26 & \text { SF2 }\end{array}$

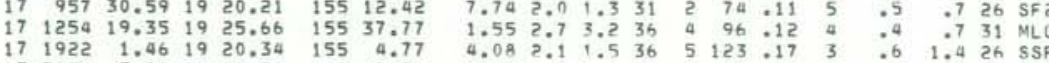

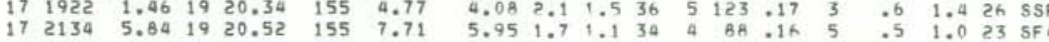

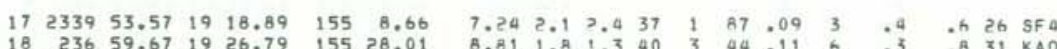

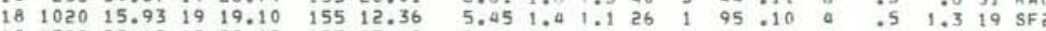

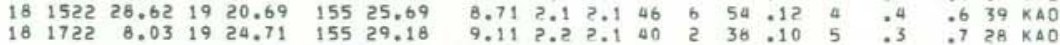

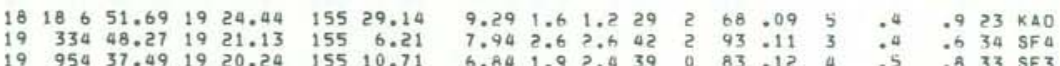

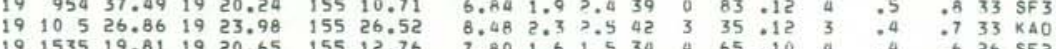

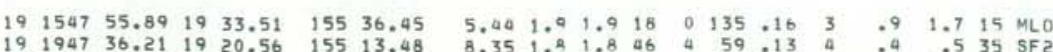

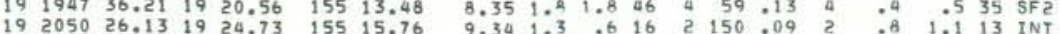

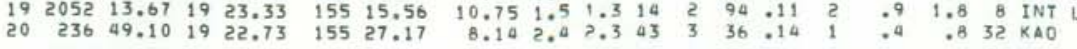

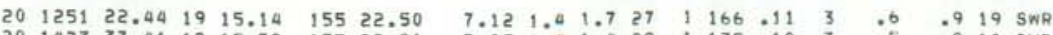

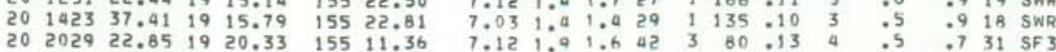




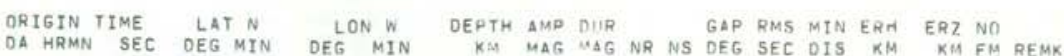

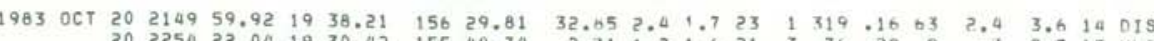
$\begin{array}{lllllllllllll} & & & \end{array}$

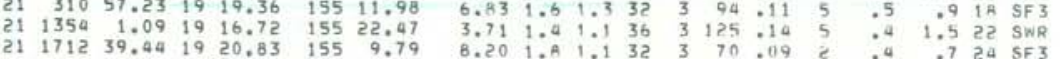

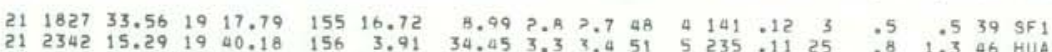

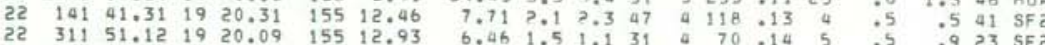

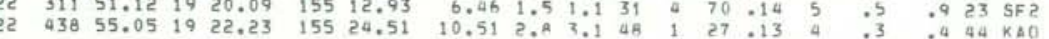

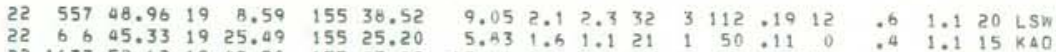

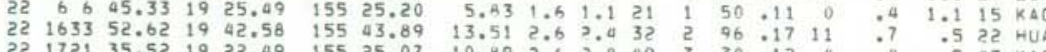

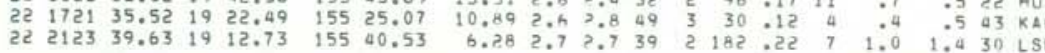

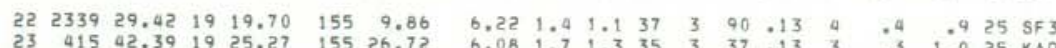

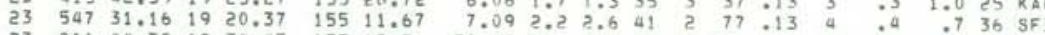

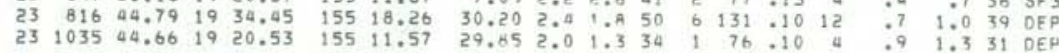

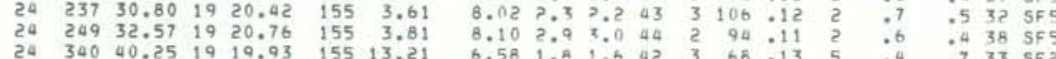

$\begin{array}{lllllllllllllllllll}24 & 1335 & 54.04 & 19 & 24.20 & 155 & 16.41 & 9.05 & 1.3 & 1.1 & 15 & 2 & 101 & .06 & 1 & .7 & .6 & 11 & \text { INT }\end{array}$

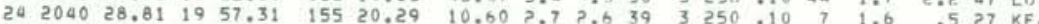

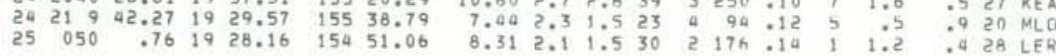

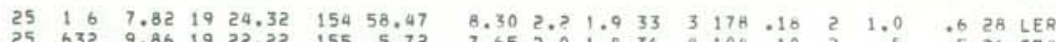

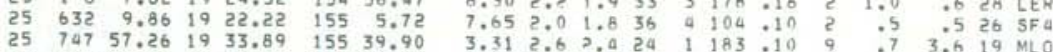

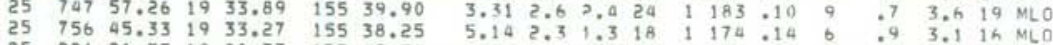

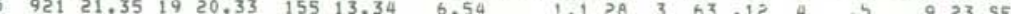

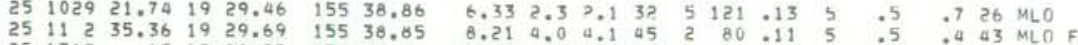

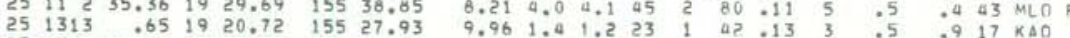

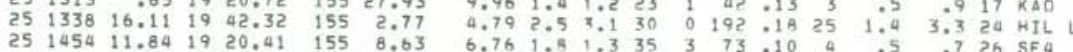

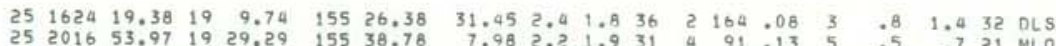

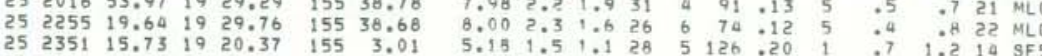

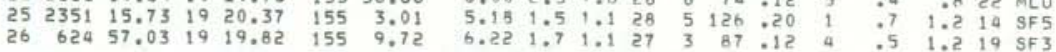

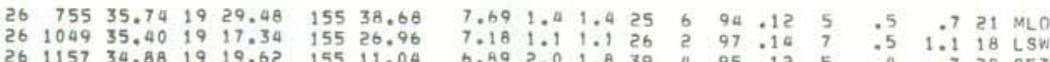

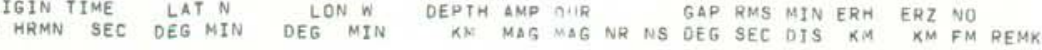

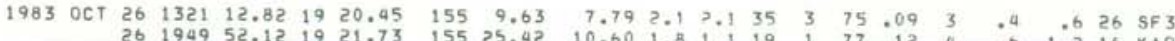

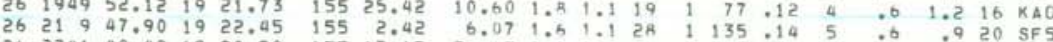

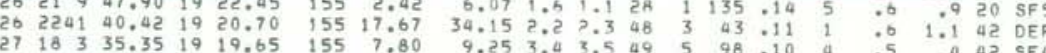

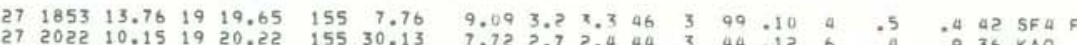

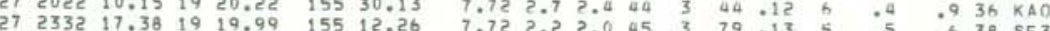

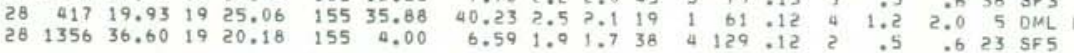
$28 \quad 1554 \quad 42.40 \quad 19 \quad 18.73 \quad 155 \quad 13.73$ $\begin{array}{lllllll}29 & 0 & 35.26 & 19 & 18.53 & 155 & 13.96 \\ 29 & 42 & 28.84 & 12.96 & \end{array}$ $\begin{array}{lllllll}29 & 421 & 28.84 & 19 & 19.09 & 155 & 14.77 \\ 29 & 447 & 59.50 & 19 & 17.57 & 155 & 22.96\end{array}$

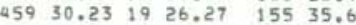

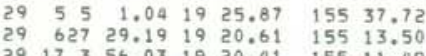
$\begin{array}{ccccccc}29 & 1926 & 9.09 & 19 & 20.87 & 155 & 2.84 \\ 30 & 19 & 48.94 & 19 & 11.50 & 155 & 30.53\end{array}$

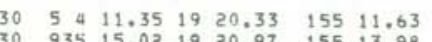

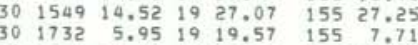
$\begin{array}{llll} & \end{array}$

$\begin{array}{lllllll}30 & 1946 & 50.55 & 19 & 21.24 & 155 & 3.33 \\ 30 & 2027 & 43.34 & 30 & 19.57 & 155 & 12.21\end{array}$ $302226 \quad 30.801925 .00 \quad 15526.71$ $\begin{array}{lllllll}31 & 832 & 38.27 & 19 & 27.08 & 155 & 27.32 \\ 31 & 1636 & 43.47 & 19 & 27.08 & 155 & 27.32\end{array}$

NoY 11.020 11.19 19 21.06

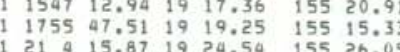

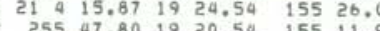

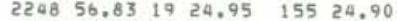

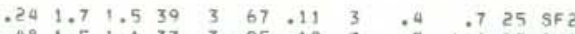

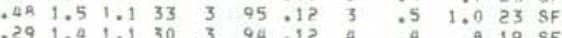

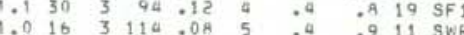

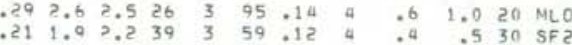

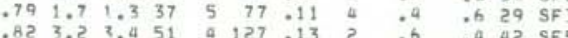

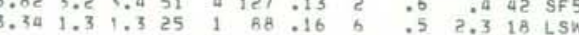

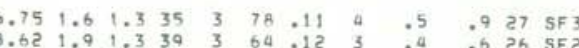

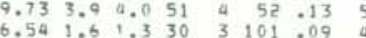

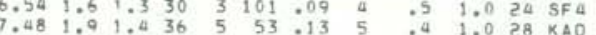

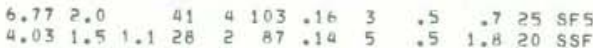

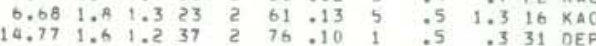

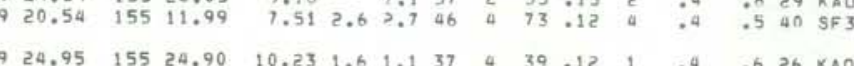

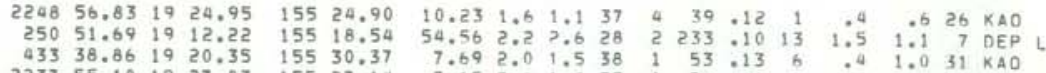

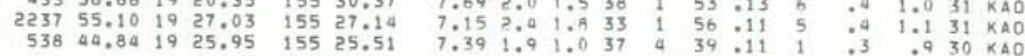

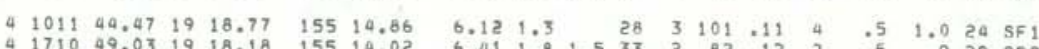

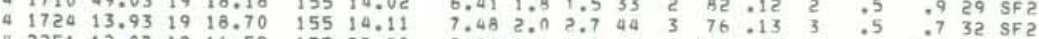

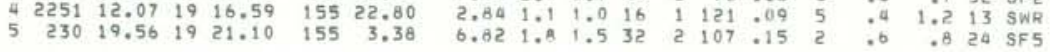

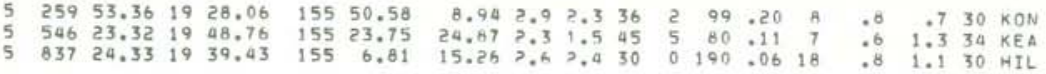


HVO EARTHQUAKE SUMMARY LIST

PAGE G1

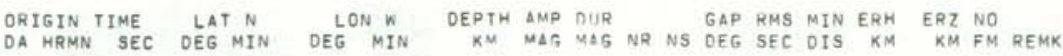

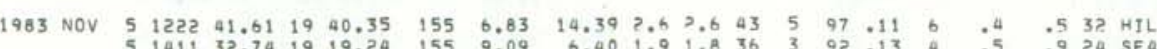

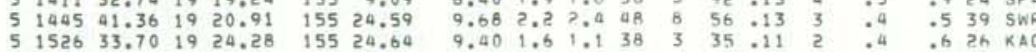

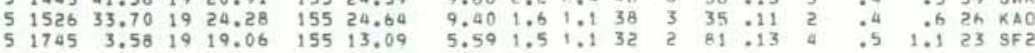

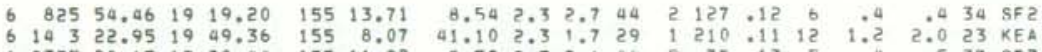

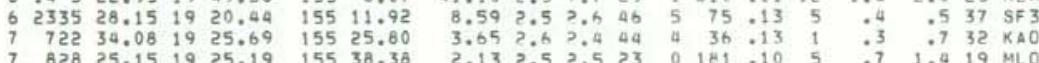

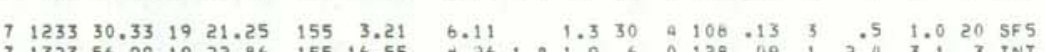

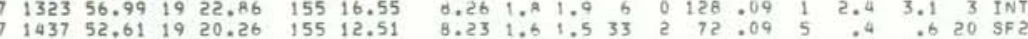

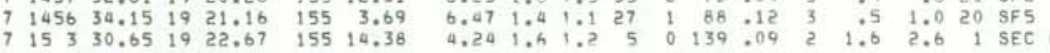

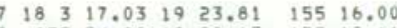
$855 \quad 54.88 \quad 1920.63 \quad 155 \quad 12.1$

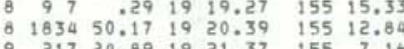

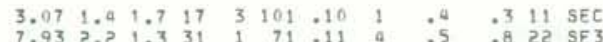

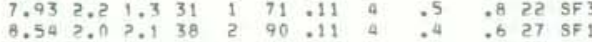

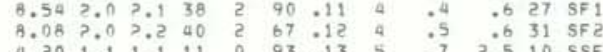

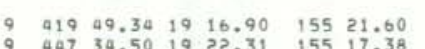
$\begin{array}{r}45420.401919 .07 \\ 45516.02 \\ \hline 1013 \quad 55.07\end{array}$

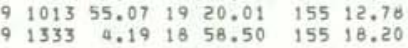
$\begin{array}{rrrrrrrr}10 & 6 & 6 & 45.76 & 19 & 20.99 & 155 & 13.16 \\ 10 & 17 & 4 & 25.49 & 19 & 19.85 & 155 & 12.92\end{array}$ $\begin{array}{rrrrrrr}11 & 755 & 43.73 & 19 & 25.94 & 155 & 37.43 \\ 11 & 914 & 3.75 & 19 & 23.66 & 155 & 20.33\end{array}$ $11.9005 \quad 6.251921 .17 \quad 155$ 26. $\begin{array}{lllllll}11 & 1459 & 33.13 & 19 & 19.66 & 155 & 15.19 \\ 11 & 1711 & 24.53 & 19 & 20.70 & 155 & 11.36\end{array}$ $11 \quad 2030 \quad 25.7919 \quad 18.28$ 155 13.90

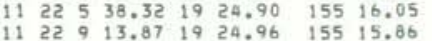
$\begin{array}{lllllll}11 & 2229 & 33.21 & 19 & 24.99 & 155 & 15.96\end{array}$

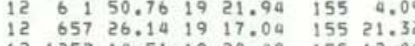

$\begin{array}{lllllll}12 & 1425 & 51.26 & 19 & 18.70 & 155 & 12.71 \\ 12 & 1627 & 56.55 & 19 & 19.80 & 155 & 11.54\end{array}$

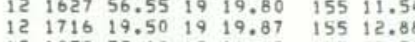

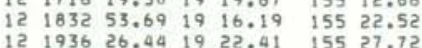
$\begin{array}{llllllll}12 & 20 & 5 & 17.61 & 19 & 19.52 & 155 & 10.83\end{array}$ $13 \quad 047 \quad 12.46 \quad 1920.98 \quad 155 \quad 3.38$
HVO EARTHQUAKE SUMMARY LIST

PAGF GP ORIGIN TIME
OA HRMN SEC DEG MIN DEG MIN DEPTH AMP OIIR N N RA GAP RMS MIN ERH ERZ NO
MAG SOG NR NS DEG SEC DIS KM KM FM REMK

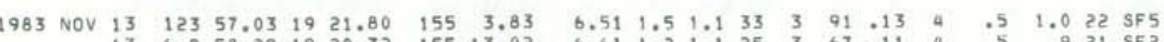

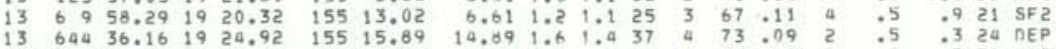

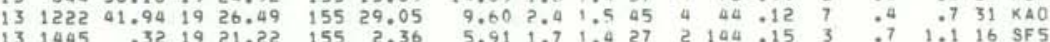

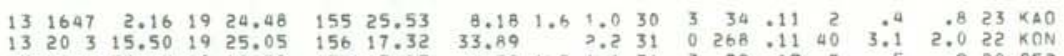

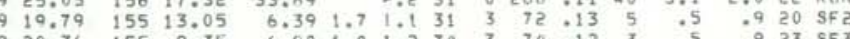

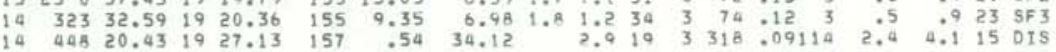

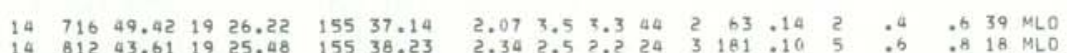

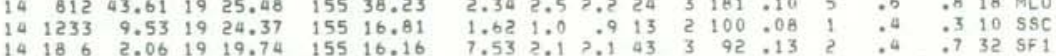

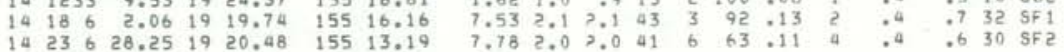
$14 \quad 2343 \quad 34.48 \quad 1921.50 \quad 155 \quad 28.31$ $15 \quad 014 \quad 12.54 \quad 1921.61 \quad 155 \quad 28.55$

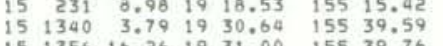

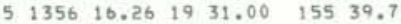
$1514348.42 \quad 1930.16 \quad 15539.58$

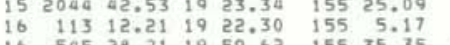

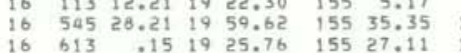

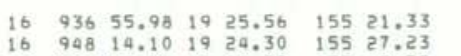

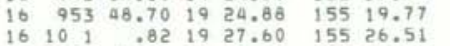

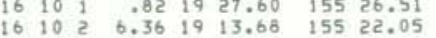

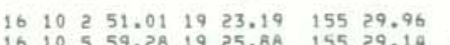

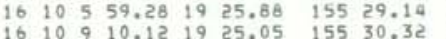
$\begin{array}{lllllll}16 & 1016 & 16.69 & 19 & 24.84 & 155 & 26.73 \\ 16 & 1016 & 46.12 & 19 & 15.11 & 155 & 30.99\end{array}$

$\begin{array}{lllllll}16 & 1020 & 2.26 & 19 & 25.28 & 155 & 21.15\end{array}$ $16 \quad 102258.611920 .50 \quad 15516.28$

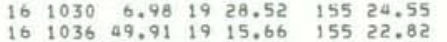
$16 \quad 1045 \quad 50,41 \quad 19 \quad 25,36 \quad 155 \quad 19,62$

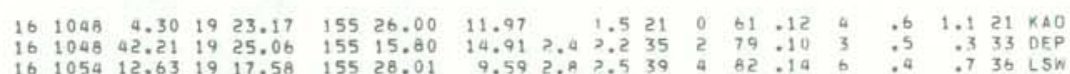

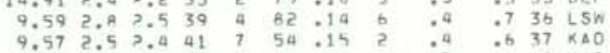
152
13.51

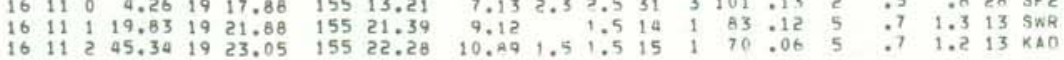




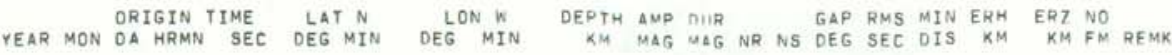

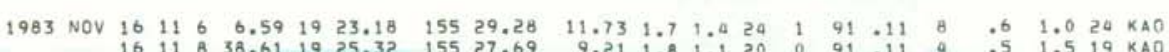

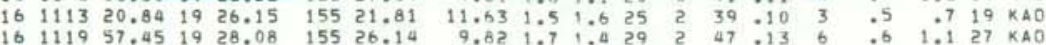

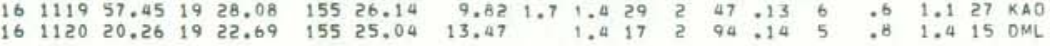

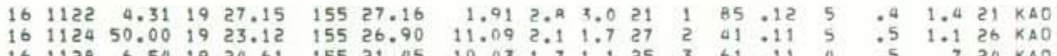

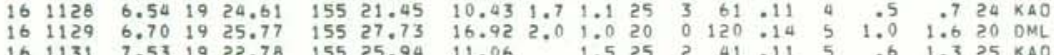
$\begin{array}{llllllllllllllllll}16 & 1133 & 22.72 & 19 & 20.86 & 155 & 23.10 & 11.22 & 1.8 & .3 & 12 & 0 & 72 & .12 & 1 & .8 & 1.3 & 9 \\ \text { SWR }\end{array}$

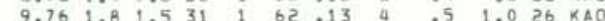

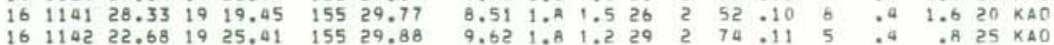

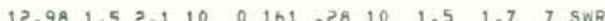

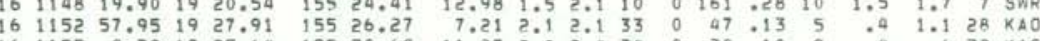

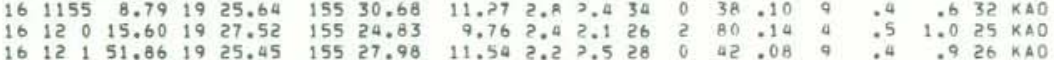

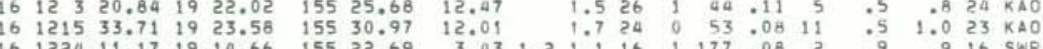

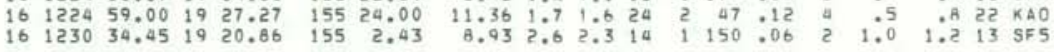

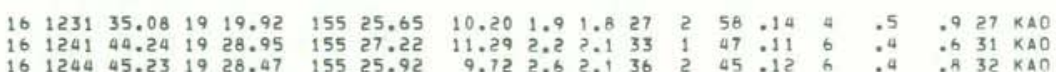

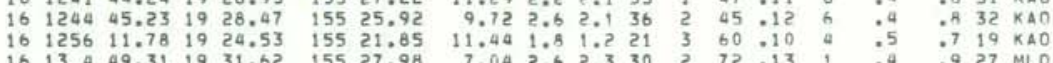

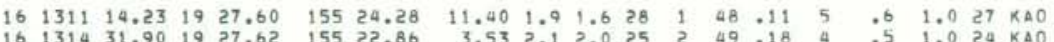

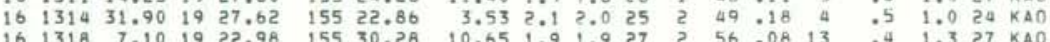

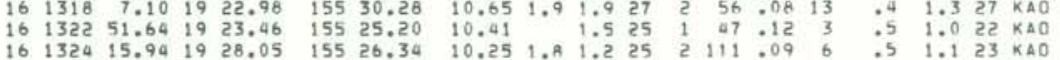

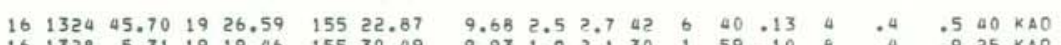

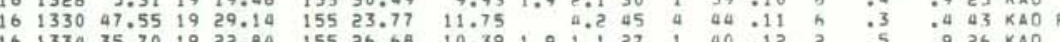

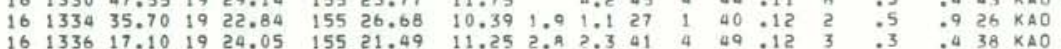

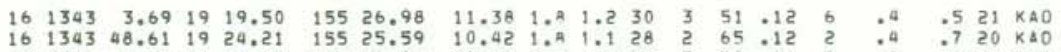

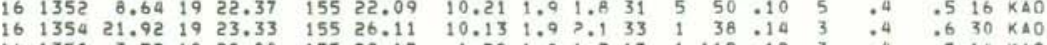

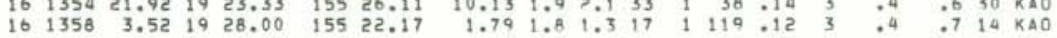

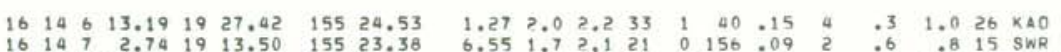

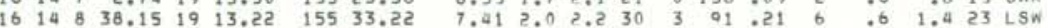

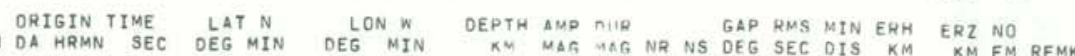

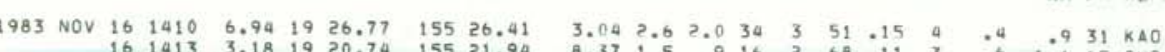

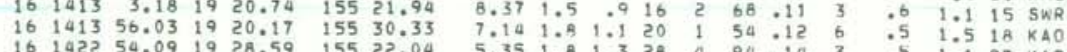

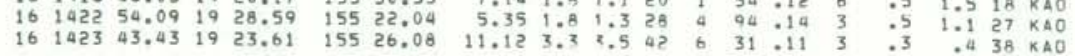
$\begin{array}{lllllll}16 & 1427 & 38.40 & 19 & 21.73 & 155 & 28.06 \\ 16 & 1439 & 14.29 & 19 & 25.69 & 155 & 20.46\end{array}$

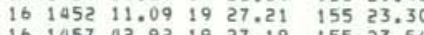

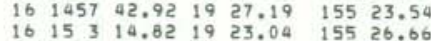
$\begin{array}{lllllllllllll}8.78 & 2.0 & 1.3 & 35 & 5 & 40 & .13 & 5 & .9 & .7 & 57 & \times 40\end{array}$

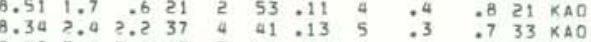

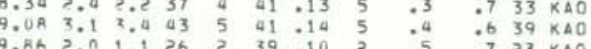

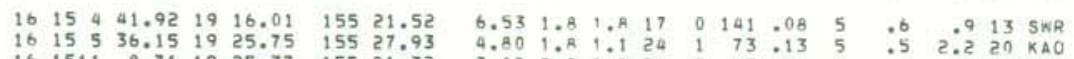

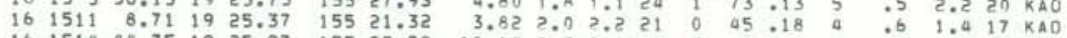

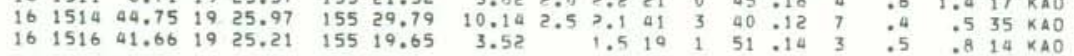

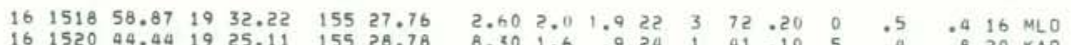

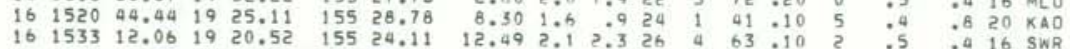

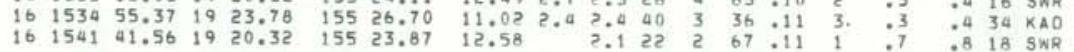

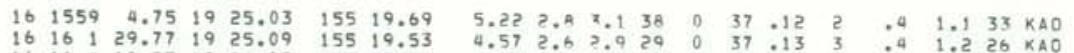

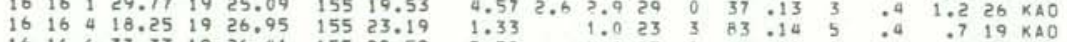

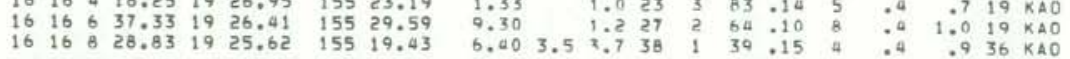

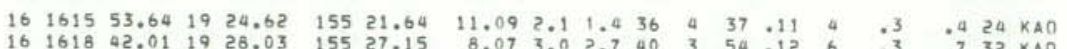

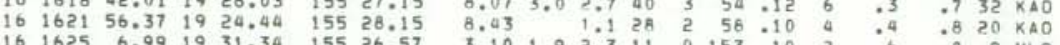

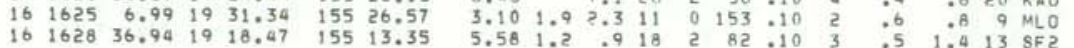
$\begin{array}{lllllllllllllllllll}16 & 1632 & 46.91 & 19 & 22.22 & 155 & 28.30 & 6.90 & 2.5 & 2.9 & 31 & 0 & 38 & .12 & 8 & .4 & 1.1 & 25 & \text { KAO } \\ 16 & 1640 & 19.84 & 19 & 29.55 & 155 & 25.94 & 2.53 & 2.8 & 2.5 & 25 & 0 & 98 & -12 & 5 & : 4 & 2.3 & 25 & k A 0\end{array}$

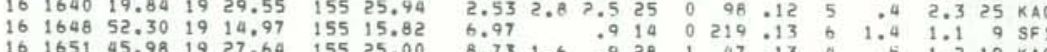

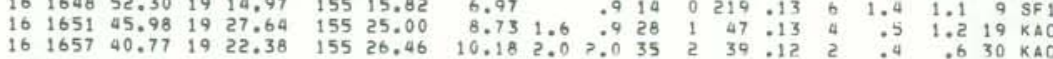

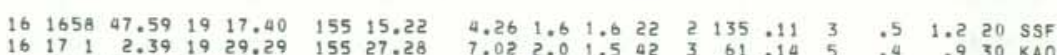

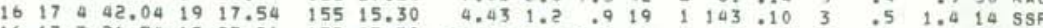

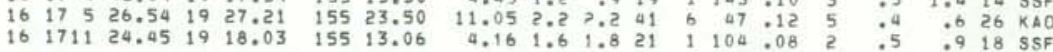

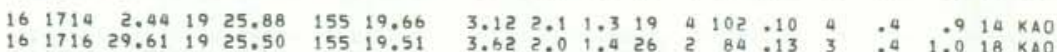

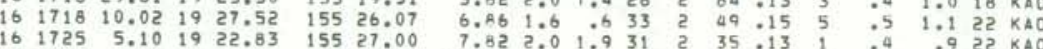

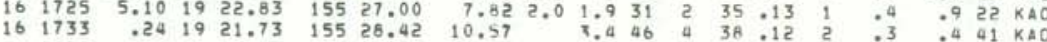

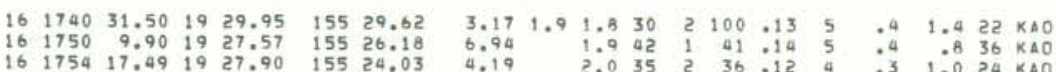


HVO EARTHQUAKE SUMMARY LIST

PAGE 65

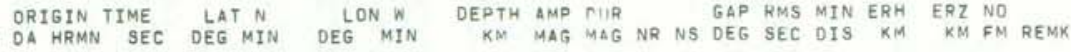

1983 NOV $16 \quad 1015$ 19.85 $1917.27 \quad 15513.57$

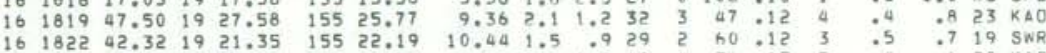

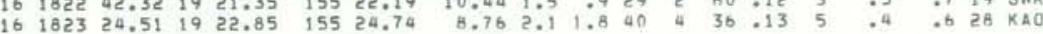

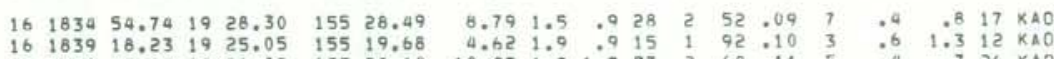
$16.1840-47.101925 .02155$.

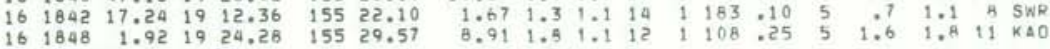

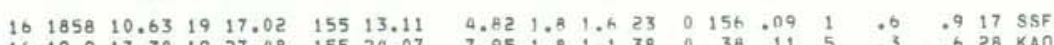

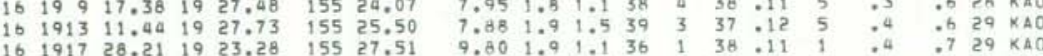

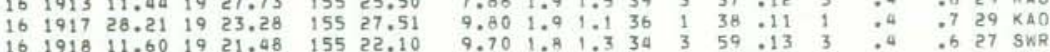

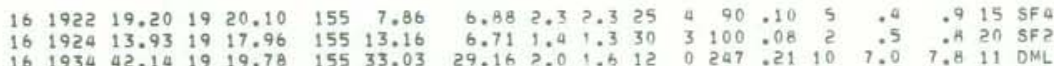

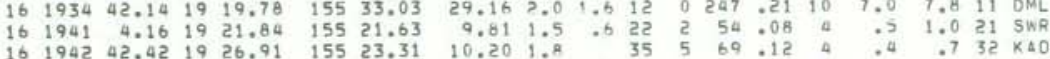
$\begin{array}{lllllllllllllllllll}16 & 1946 & 30.06 & 19 & 22.91 & 155 & 26.01 & 10.72 & 1.7 & 1.5 & 34 & 4 & 40 & .11 & 3 & .4 & .7 & 32 & \times 40\end{array}$

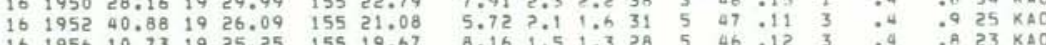

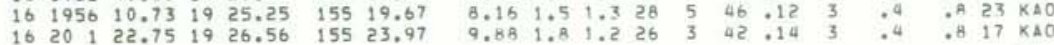
$16 \quad 207 \quad 17.051928 .14 \quad 15525.10$ 16 2026 $54.061928 .06 \quad 15521.35$ 16203037.28 19 $28.42 \quad 15526.19$

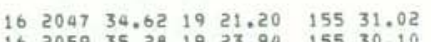
16.

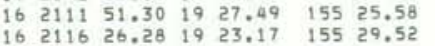

$\begin{array}{lllllll}16 & 2117 & 23.43 \quad 19 & 30.07 \quad 155 \quad 27.18\end{array}$

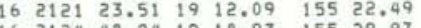

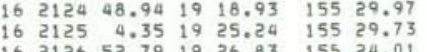

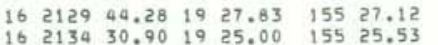
16 .1140 1.70

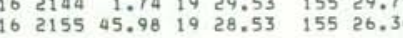
$\begin{array}{lllllll}16 & 2158 \quad 7.77 \quad 19 & 14.05 \quad 155 & 21.27\end{array}$ 16 22 $4 \quad 6.77 \quad 1925.01 \quad 155 \quad 19,30$
HVO EARTHQUAKE SUMMARY LIST

PAGE 66

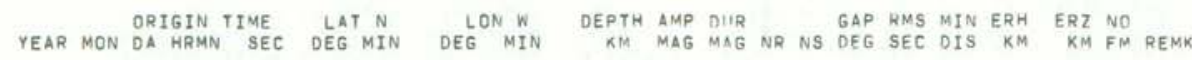

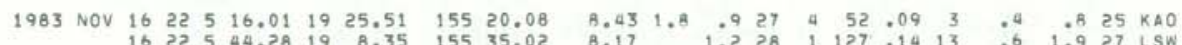

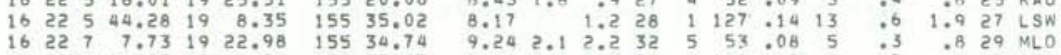

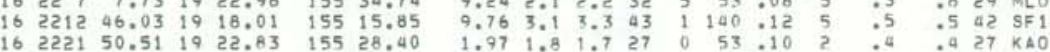
$\begin{array}{llllllllllllrllllll}16 & 2223 & 25.27 & 19 & 26.57 & 155 & 22.86 & 6.82 & 1.9 & 1.7 & 34 & 4 & 40 & .12 & 4 & .3 & .8 & 30 & K A O\end{array}$

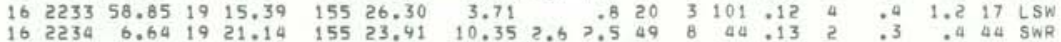

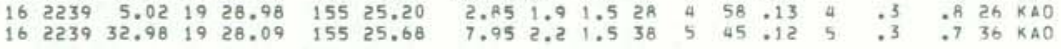

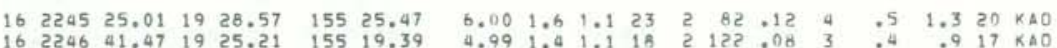

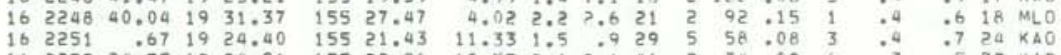

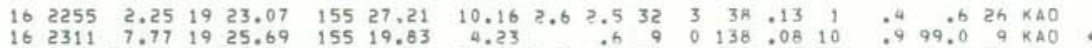

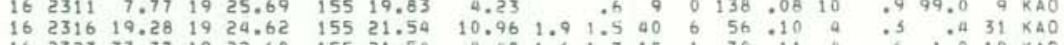

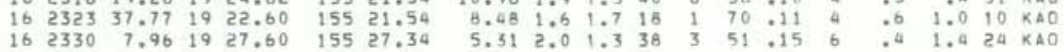

$\begin{array}{llllllllllllllllllll}16 & 2353 & 2.39 & 19 & 21.55 & 155 & 22.31 & 10.48 & 1.8 & 1.7 & 35 & 4 & 58 & .14 & 3 & .4 & .5 & 26 & \text { SWR }\end{array}$

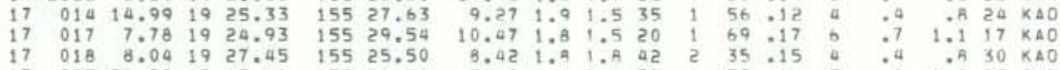

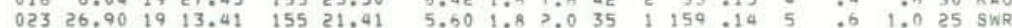

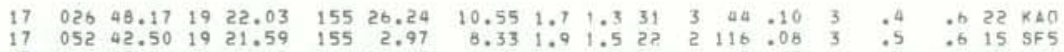

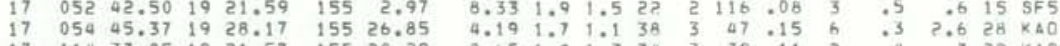

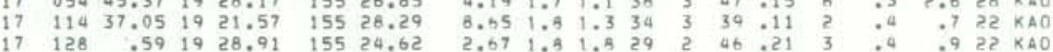
$\begin{array}{lllllllllllllllllll}17 & 130 & 32.78 & 19 & 27.67 & 155 & 26.15 & 7.02 & 2.0 & 1.5 & 43 & 3 & 41 & .12 & 5 & .3 & .8 & 30 & \text { KAO }\end{array}$

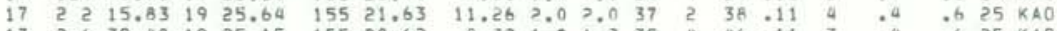

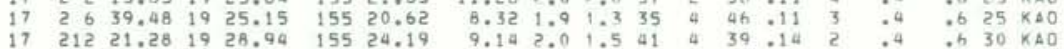

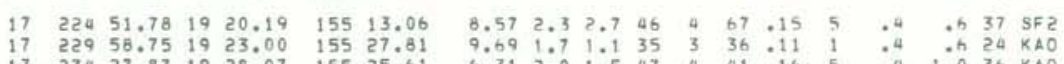

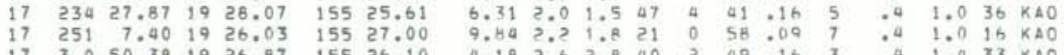

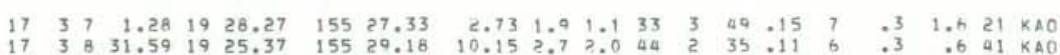

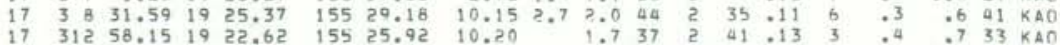

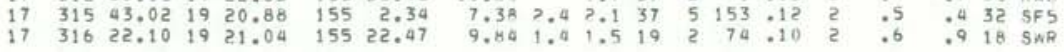

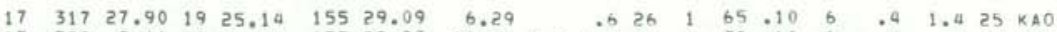

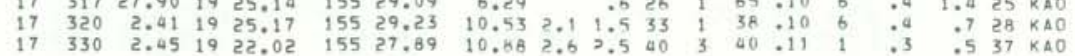


HVO EARTHQUAKE SUMMARY LIST

PAGE 67

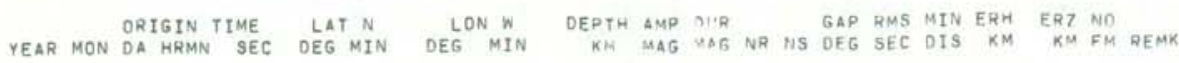

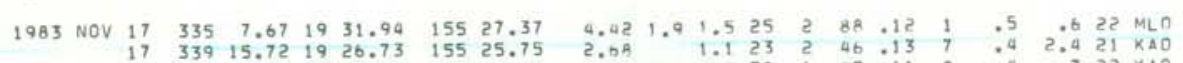

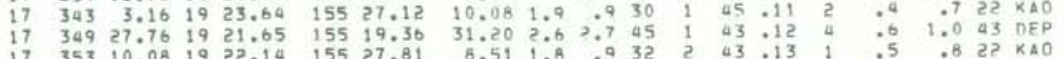

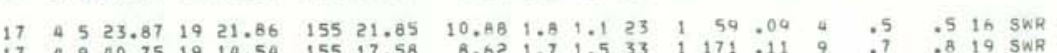

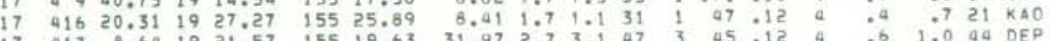

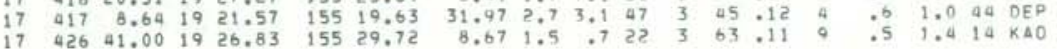
$\begin{array}{lllllllllllllllllllll}17 & 431 & 22.24 & 19 & 30.16 & 155 & 27.52 & 5.33 & 1.5 & .5 & 19 & 2 & 80 & 08 & 3 & .4 & 1.4 & 14 & \text { MLO } \\ 17 & 437 & 22.19 & 19 & 26.12 & 155 & 30.27 & 8.45 & 2.5 & 1.5 & 36 & 2 & 41 & 11 & 8 & .3 & .9 & 29 & \text { KAO }\end{array}$

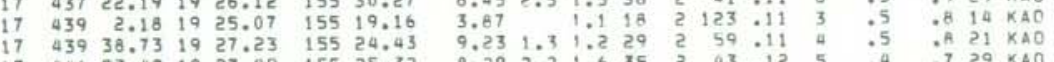

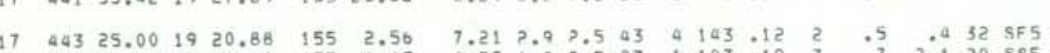

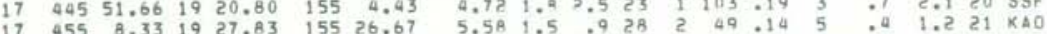

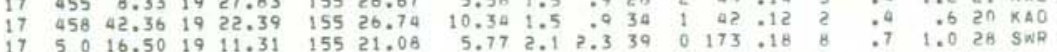

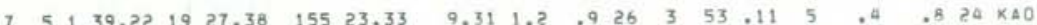

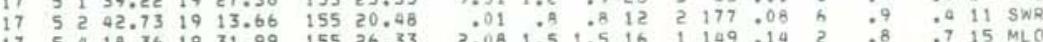

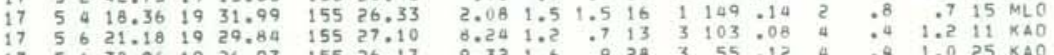

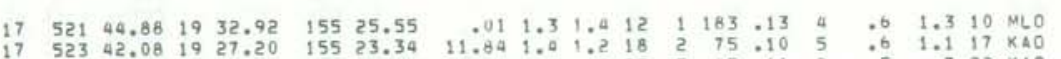

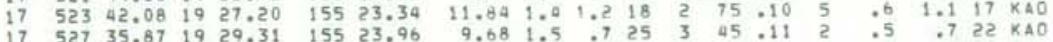

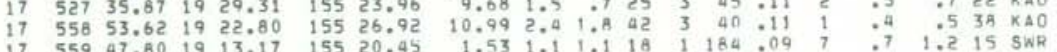

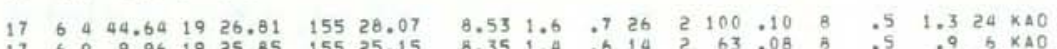

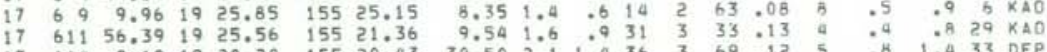

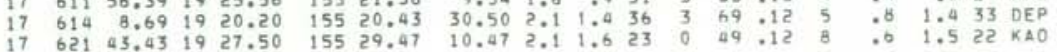
$\begin{array}{lllllllllllllllllll}17 & 624 & 13.78 & 19 & 27.59 & 155 & 26.01 & 8.05 & 1.8 & .9 & 31 & 2 & 76 & .13 & 5 & .4 & .9 & 28 & K A 0 \\ 17 & 629 & 4.20 & 19 & 20.87 & 155 & 29.99 & 5.74 & 1.4 & 1.3 & 21 & 1 & 53 & .10 & 5 & .4 & 1.6 & 20 & K A 0\end{array}$

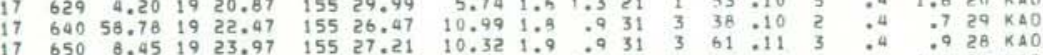

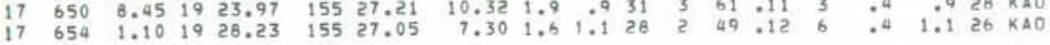

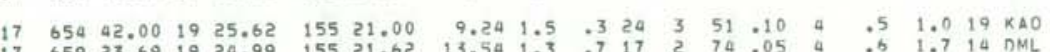

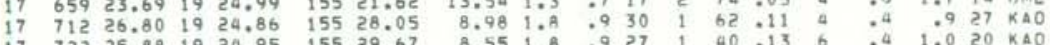

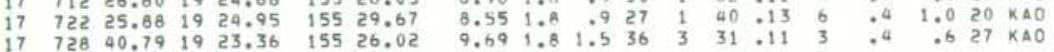

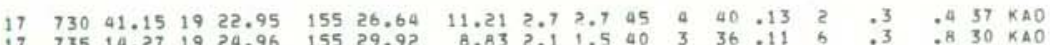

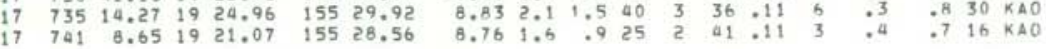

HVO EARTHOUAKE SUMMARY LIST

PAGE 68

ORIGIN TIME LAT N LON W DEPTH AMP RUR GAP RMS MIN ERH ERZ NO

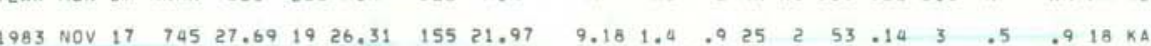

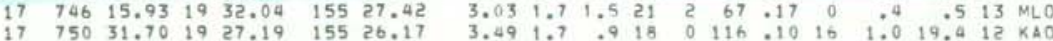

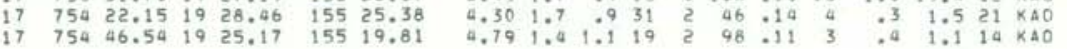

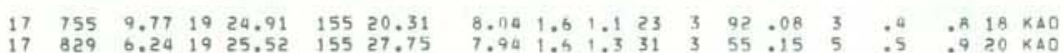

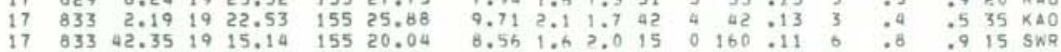

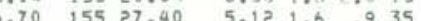

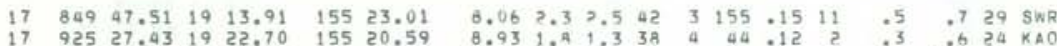

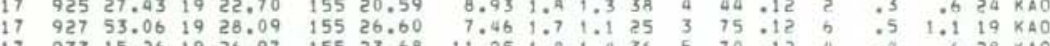

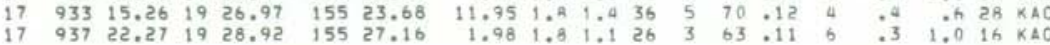

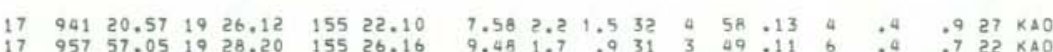

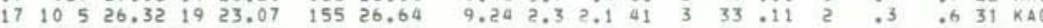

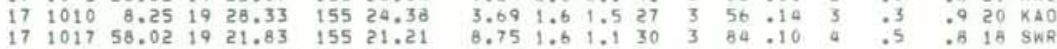

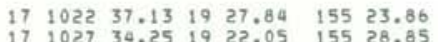

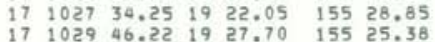

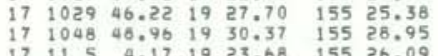
$\begin{array}{llllllll}17 & 11 & 6 & 40.55 & 19 & 25.54 & 155 & 28.54\end{array}$ $17 \quad 1114 \quad 47.67 \quad 19 \quad 27.74 \quad 15526.92$ $17111536.921921 .93 \quad 15526,46$

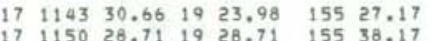

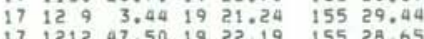
$171215 \quad 1.96 \quad 1924,99 \quad 155 \quad 19.84$

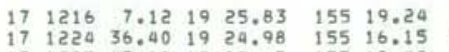
1771225 45.62 $1925.67 \quad 15519.48$

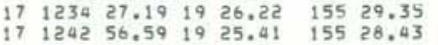
$\begin{array}{llllllll}17 & 1247 & 37.07 & 19 & 25.01 & 155 & 19.21\end{array}$

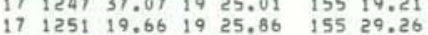

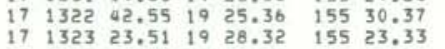

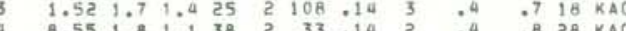

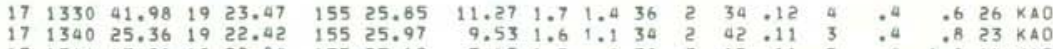


HVO EARTHQUAKE SUMMARY LIST

PAGE 69

ORIGIN TIME LAT N LON W DEPTH AMP OIR N GAP RMS MIN ERH ERZ NO

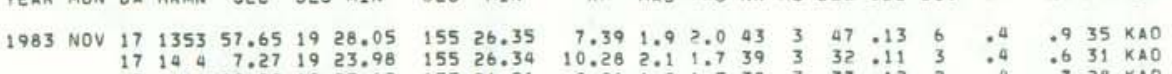

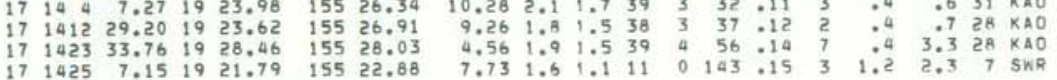

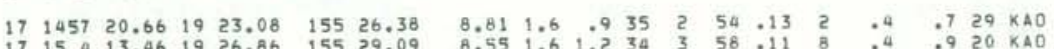

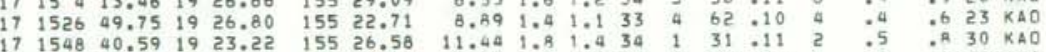

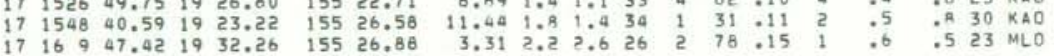

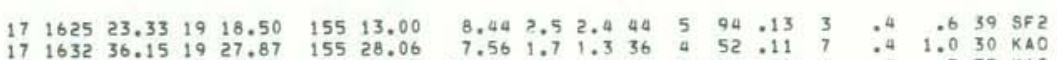

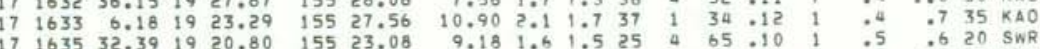

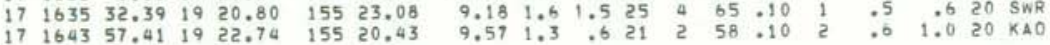

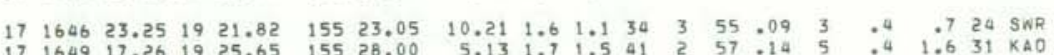

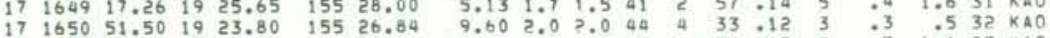

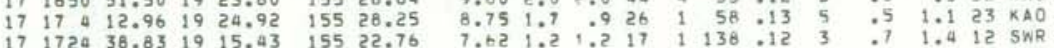

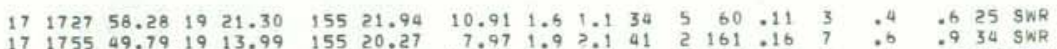

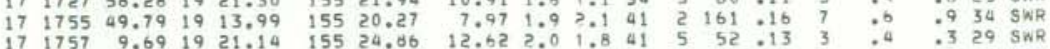

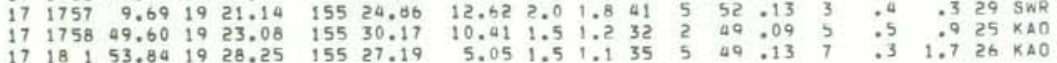

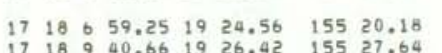
17 1820 25.58 19 25. 16 155 19.53

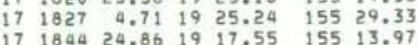
$\begin{array}{lllllll}17 & 1848 & 2.42 & 19 & 24.86 & 155 & 20.73\end{array}$

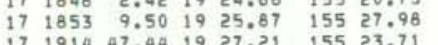

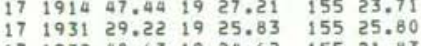

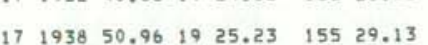
$17 \quad 1939 \quad 40.901927 .45 \quad 15525.54$

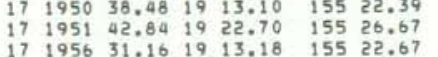

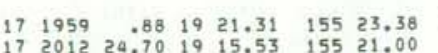

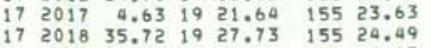

c)

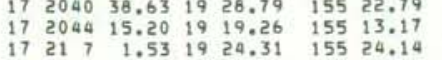

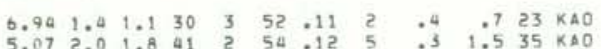

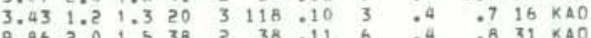

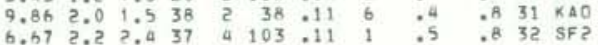
$\begin{array}{llllllllllllll}9.47 & 1.6 & 1.1 & 37 & 5 & 45 & -10 & 3 & .4 & .5 & 22 & K A 0 \\ 0.58 & .6 & .1 & 34 & 2 & 40 & 013 & 5 & 4 & 1.2 & 29 & K A O\end{array}$

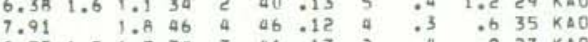
$\begin{array}{lllllllllllll}8.57 & 1.7 & .5 & 34 & 3 & 41 & 13 & 2 & .4 & .9 & 27 & K A O \\ 9.44 & 1.6 & 1.5 & 40 & 5 & 37 & .12 & 4 & .4 & .6 & 34 & K A O\end{array}$

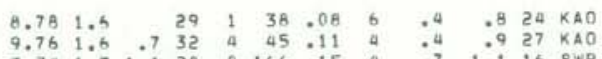

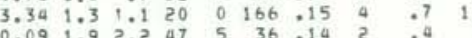

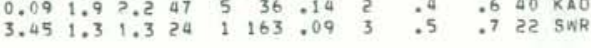
$\begin{array}{rrrrrrrrrrrr}1.18 & 1.5 & .1 & 30 & 5 & 48 & 10 & 2 & .4 & .7 & 25 & \text { SWR } \\ 7.47 & 1.2 & 1.7 & 29 & 0 & 147 & .13 & 6 & 06 & 1.1 & 27 & \text { SNR }\end{array}$

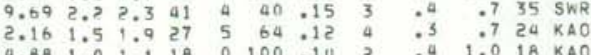
$\begin{array}{llllllllllll}3.15 & 1.3 & 1.5 & 21 & 2 & 142 & .14 & 2 & .7 & .6 & 19 & \mathrm{KAC}\end{array}$

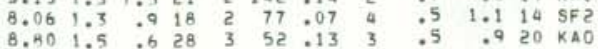

HVO EARTHQUAKE SUMMARY LIST

PAGE 70

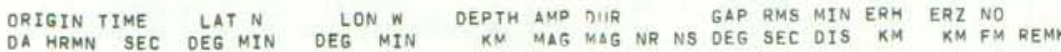

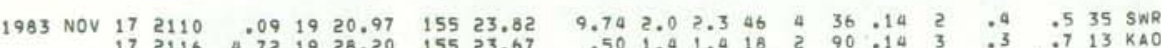

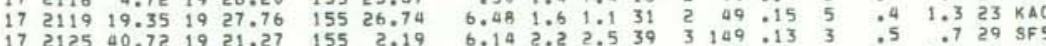

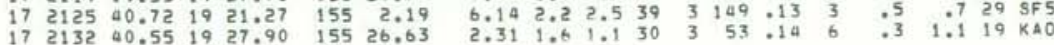

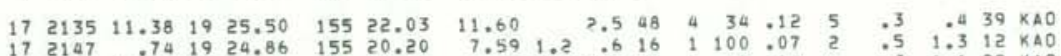

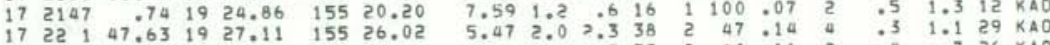

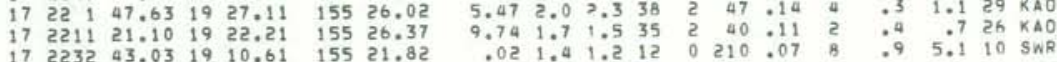

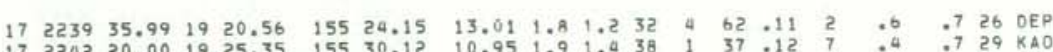

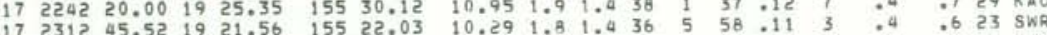

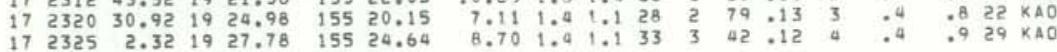

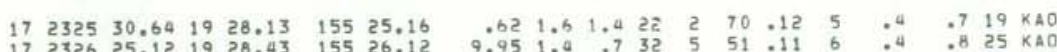

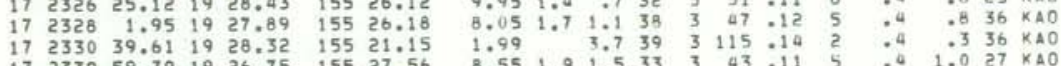

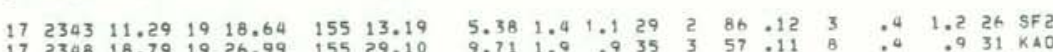

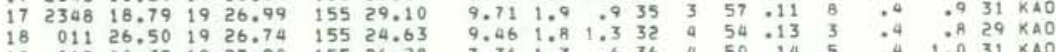

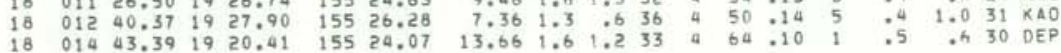

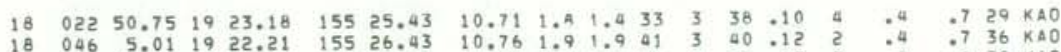

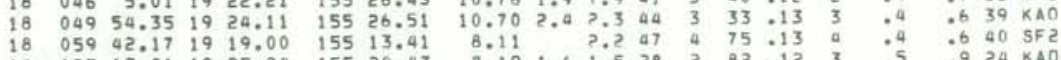
$\begin{array}{llllllllllllll}4 & 13 & 6 & .4 & .5 & 37 & k A O\end{array}$

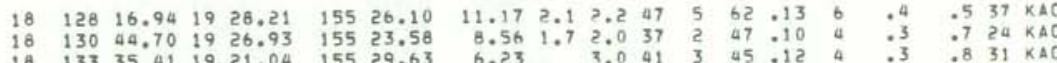

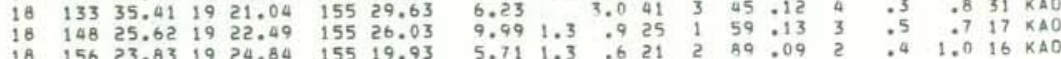

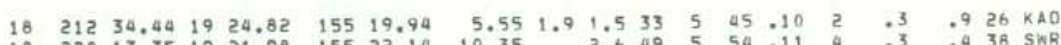

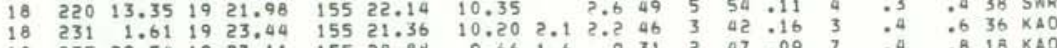

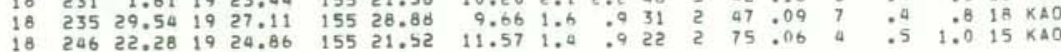

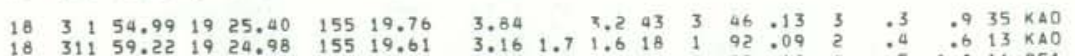

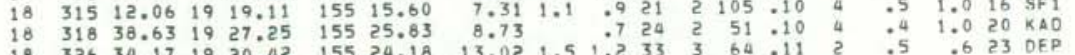
$\begin{array}{llllllllllllllllllll}18 & 418 & 33.91 & 19 & 27.06 & 155 & 24.16 & 7.89 & 1.4 & 1.1 & 29 & 4 & 71 & .15 & 4 & .4 & 1.0 & 17 \quad \text { KAD }\end{array}$

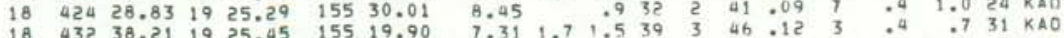




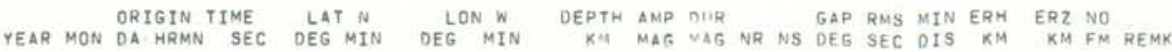

1983 NOV 18 439 $1.641926 .74 \begin{array}{llllllllllllll}155 & 23.75 & 10.43 & 1.5 & .2 & 26 & 4 & 65 & .13 & 4 & .4 & 77 & 17 & K 40\end{array}$

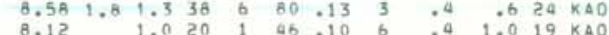

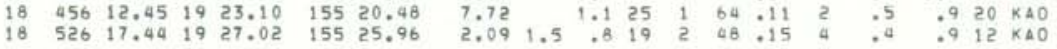

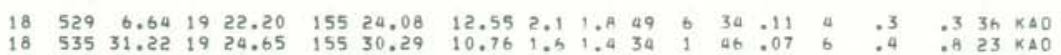

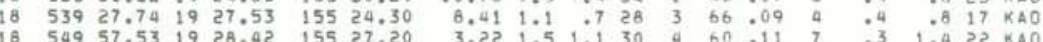

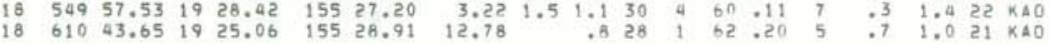

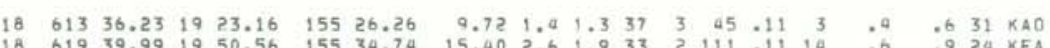

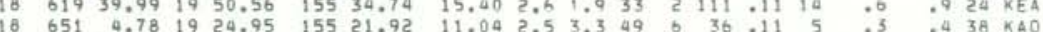

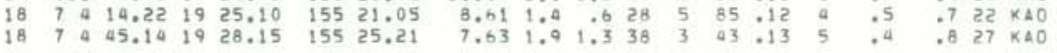
$\begin{array}{llllllllllllllllllll}18 & 7 & 5 & 24.88 & 19 & 23.24 & 155 & 25.82 & 9.81 & 2.1 & 2.0 & 40 & 2 & 30 & .12 & 3 & .4 & .7 & 30 & \text { KAO }\end{array}$

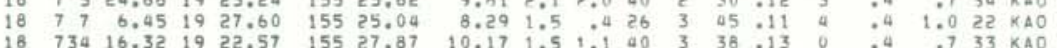

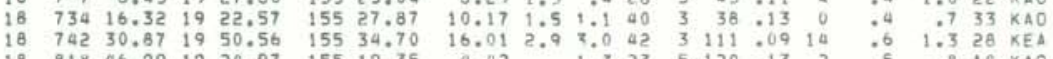

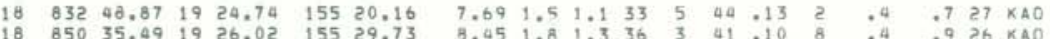

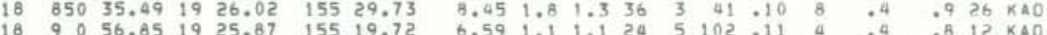

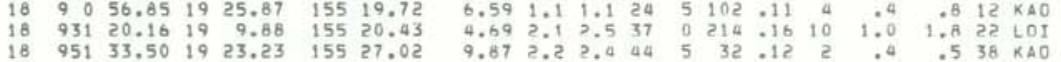
$\begin{array}{llllllllllllllllll}18 & 954 & 38.15 & 19 & 25.93 & 155 & 19.91 & 3.83 & 2.2 & 2.6 & 32 & 1 & 47 & .13 & 4 & .4 & 1.3 & 23 \\ \mathrm{KAO}\end{array}$

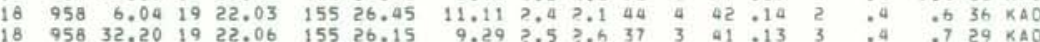

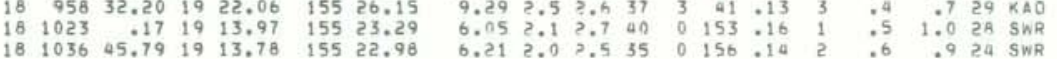
$\begin{array}{rrrrrrrrrrrrrrrrrrr}18 & 1052 & 7.23 & 19 & 18.05 & 155 & 23.77 & 8.18 & 1.7 & 1.9 & 33 & 2 & 89 & .14 & 9 & .5 & .9 & 21 & \text { SWR } \\ 18 & 1059 & 46.07 & 19 & 29.20 & 155 & 24.84 & 1.42 & 1.7 & 2.0 & 23 & 4 & 88 & .15 & 3 & .3 & .8 & 20 & K 40\end{array}$

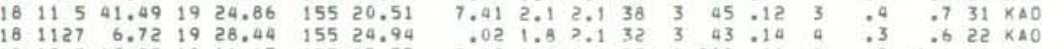

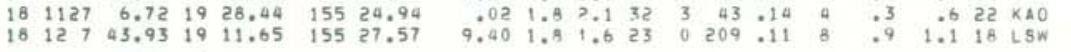

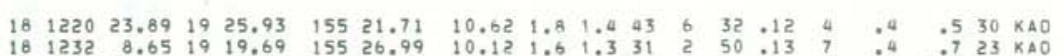

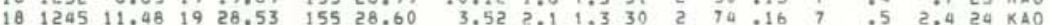

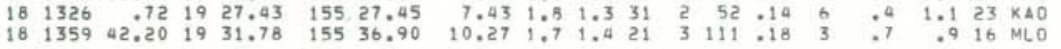

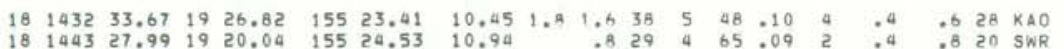

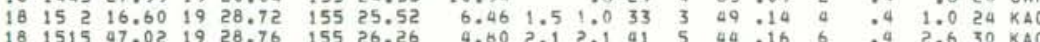

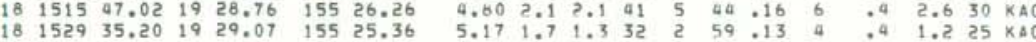

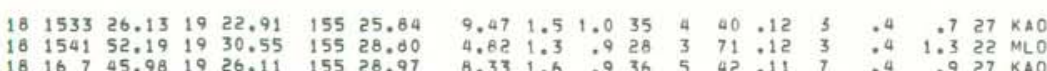

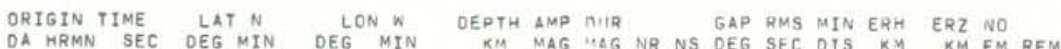

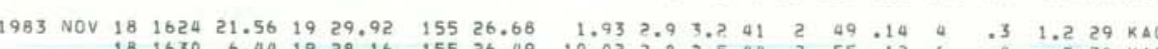

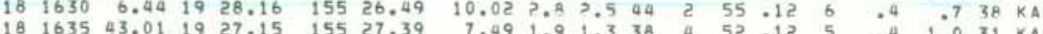

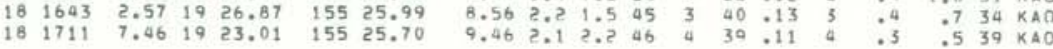

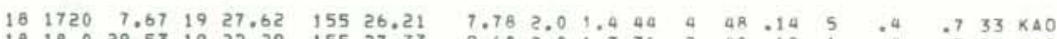

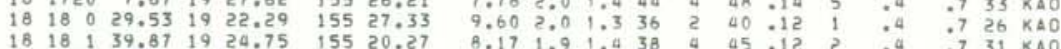

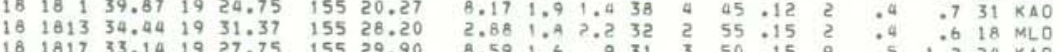

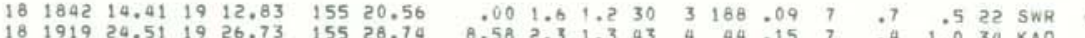

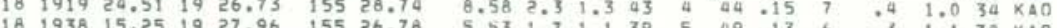

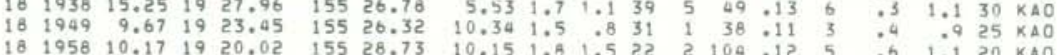

$\begin{array}{llllllllllllllllllll}18 & 2024 & 15.37 & 19 & 27.56 & 155 & 28.01 & 9.35 & 1.6 & 1.2 & 24 & 3 & 78 & .10 & 7 & .5 & 1.1 & 20 & \mathrm{kAO}\end{array}$

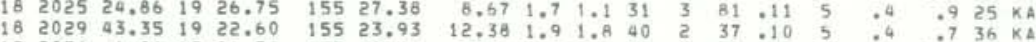

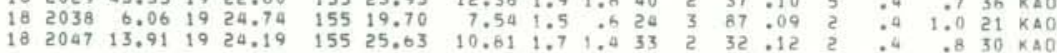

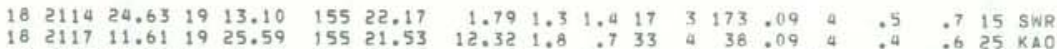

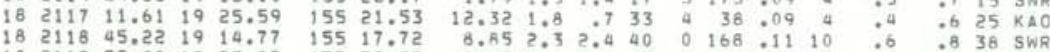

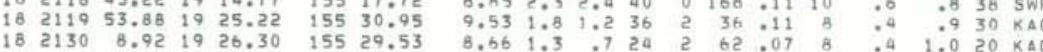

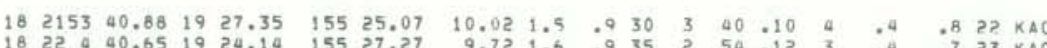

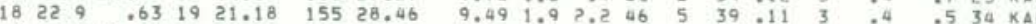

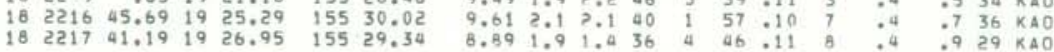

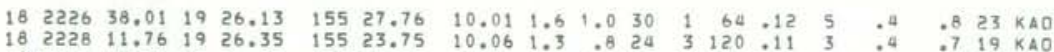

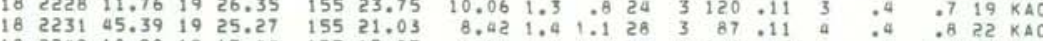

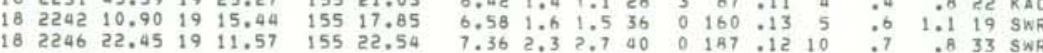

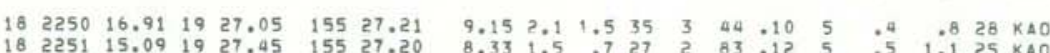

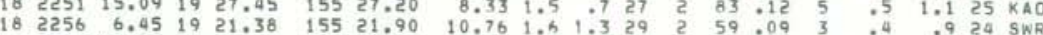

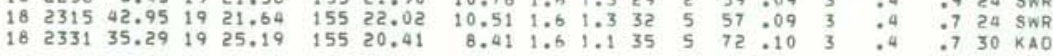

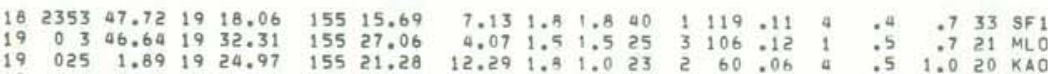

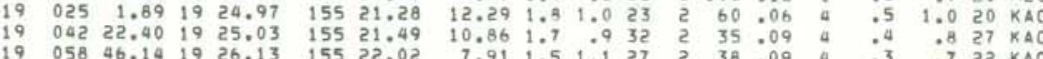

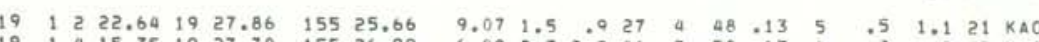

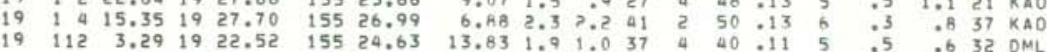




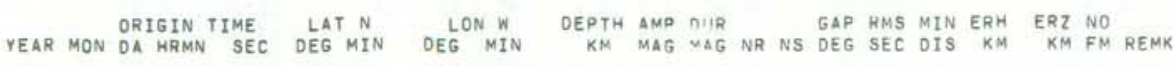

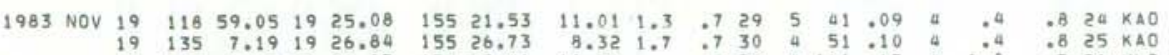

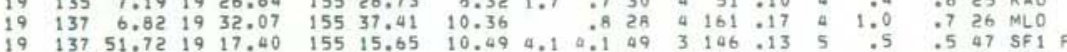

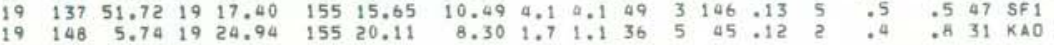

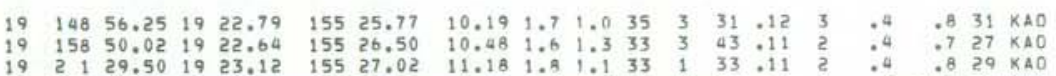

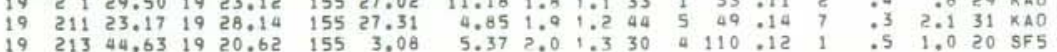

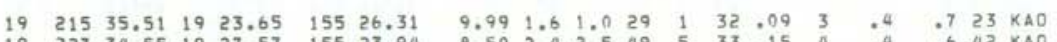

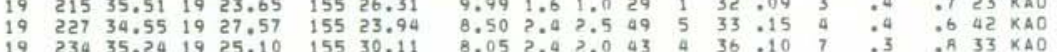

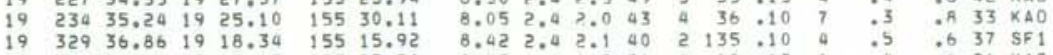

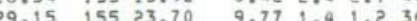
$\begin{array}{rrrrrrrrrrrrrrrrrrr}19 & 354 & 9.39 & 19 & 18.05 & 155 & 15.86 & 6.16 & 1.8 & 1.5 & 35 & 0 & 132 & .12 & 5 & .5 & .9 & 30 & \text { SF } 1 \\ 19 & 420 & 13.60 & 19 & 23.42 & 155 & 26.02 & 10.89 & 1.6 & 1.2 & 33 & 2 & 37 & .09 & 3 & : .4 & .5 & 26 & \text { KAO }\end{array}$

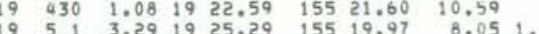

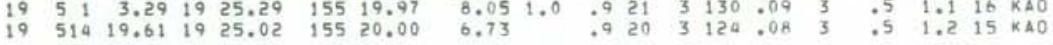

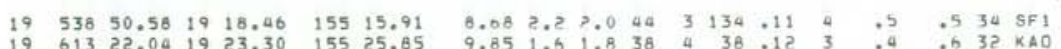

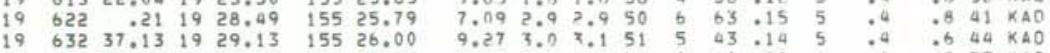

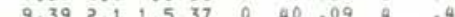

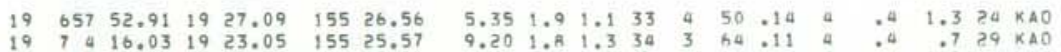

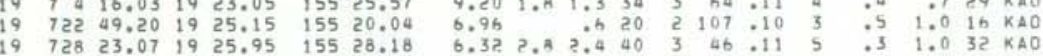

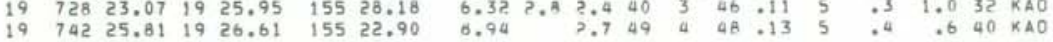

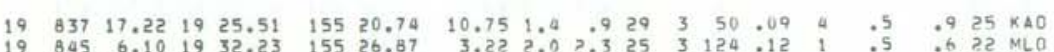

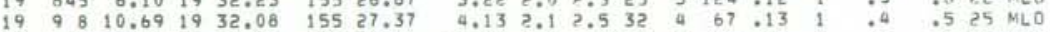

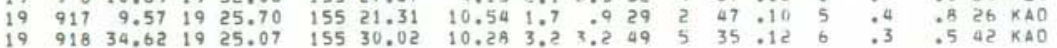

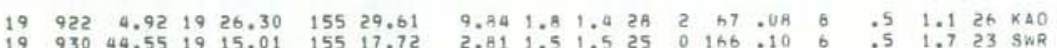

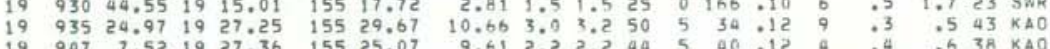

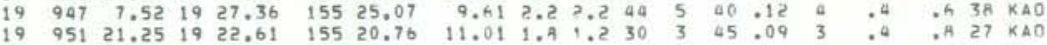
$\begin{array}{lllllllllllllllllll}19 & 1121 & 12.34 & 19 & 28.05 & 155 & 26.91 & 3.31 & 1.2 & .0 & 35 & 4 & 63 & .13 & 6 & .4 & 1.5 & 30 & \text { KAO }\end{array}$

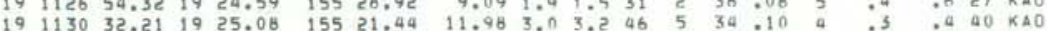

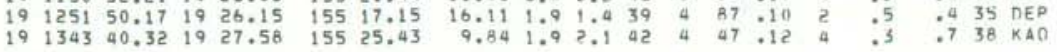

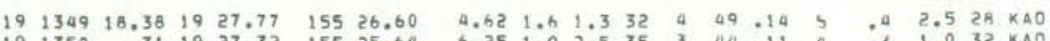

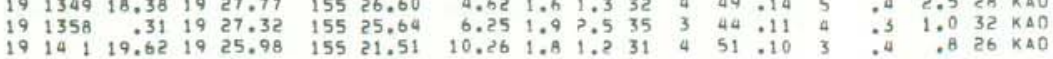

PAGE 74 ORIGIN TIME LAT N
LON N

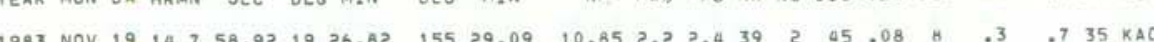

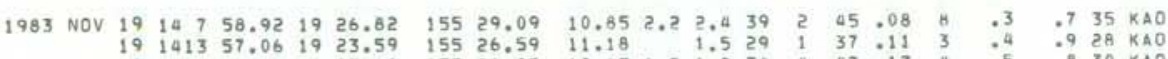

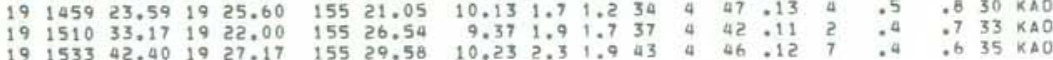

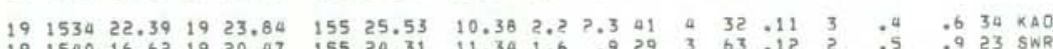

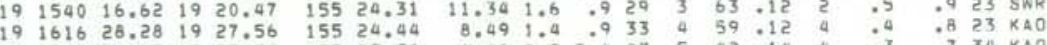

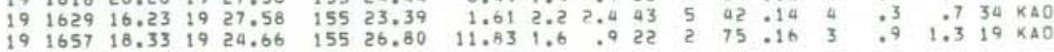

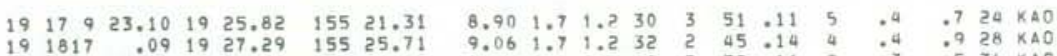

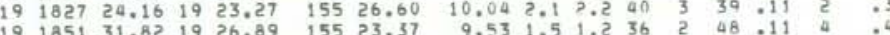

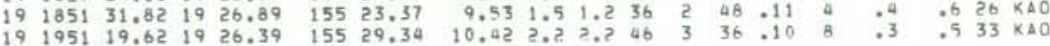

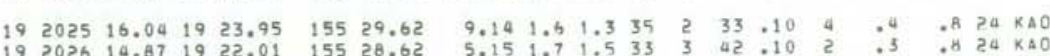

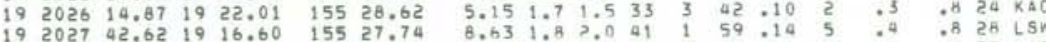

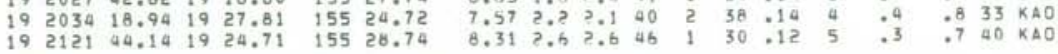

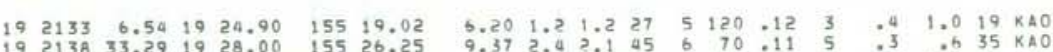

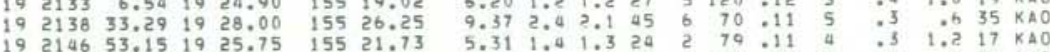

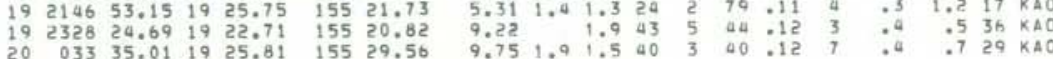

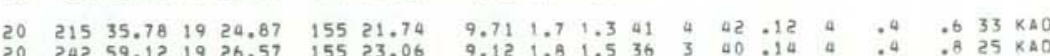

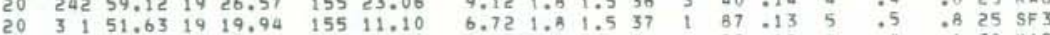

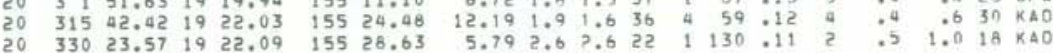

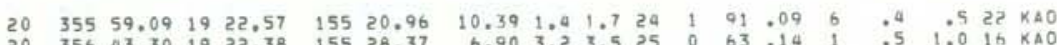

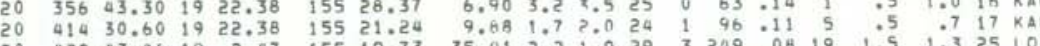

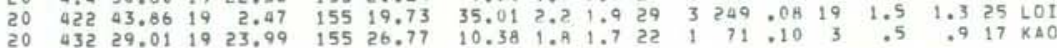

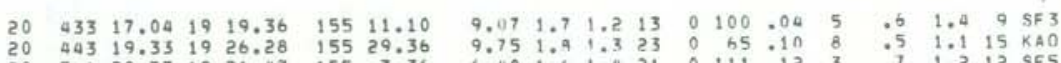

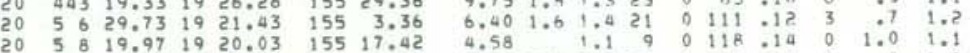

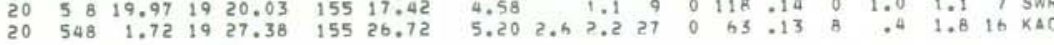

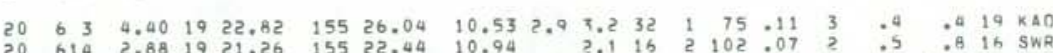

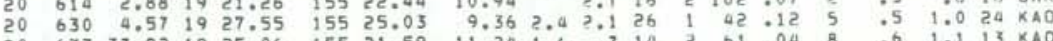

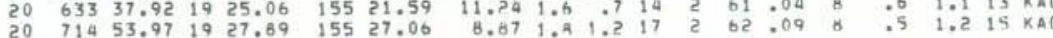

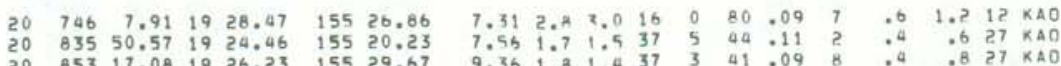




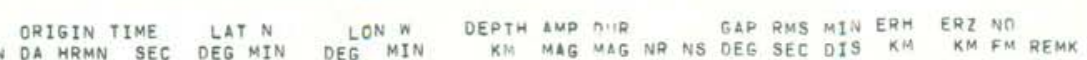

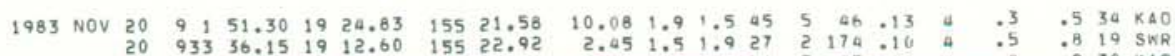

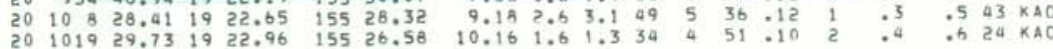
$\begin{array}{llllllllllllllllll}20 & 1030 & 48.08 & 19 & 25.66 & 155 & 20.00 & 8.78 & 1.7 & 1.1 & 30 & 4 & 118 & .10 & 4 & .4 & .7 & 21 \\ K A O & 0\end{array}$

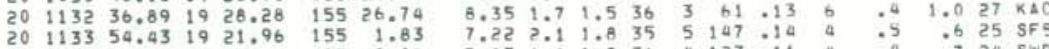

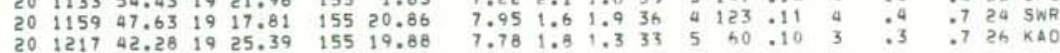
$\begin{array}{lllllllllllllllllll}20 & 1233 & 12.50 & 19 & 21.18 & 155 & 22.34 & 9.53 & 1.5 & 1.3 & 18 & 2 & 91 & .10 & 2 & .8 & 1.0 & 12 & \text { SWR } \\ 20 & 12 & .3 & 31 & \text { KAO }\end{array}$

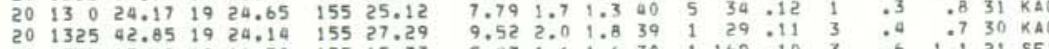

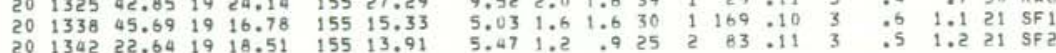

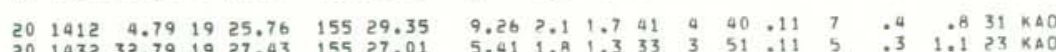

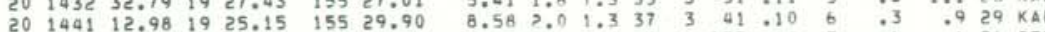

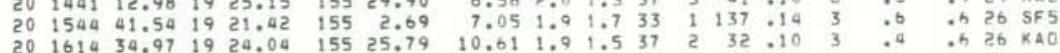

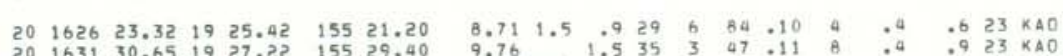

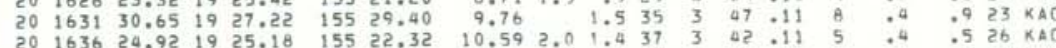

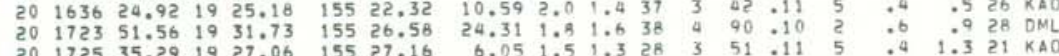

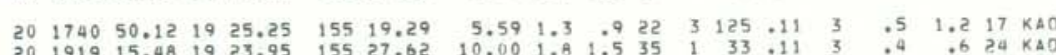

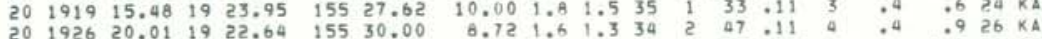

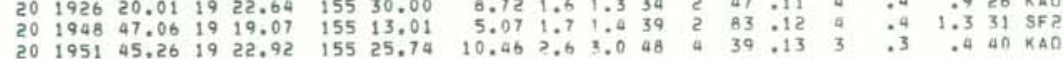
$\begin{array}{llllllllllllllllll}20 & 1956 & 23.24 & 19 & 24.65 & 155 & 21.42 & 10.46 & 1.7 & 1.4 & 39 & 4 & 36 & .12 & 4 & .4 & .5 & 29 \\ 2 A 0\end{array}$

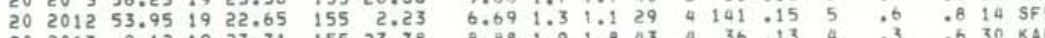

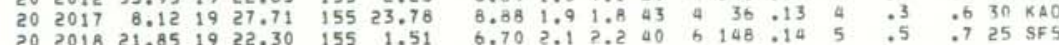

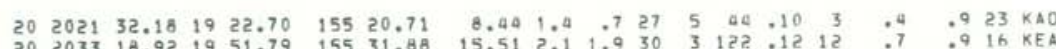

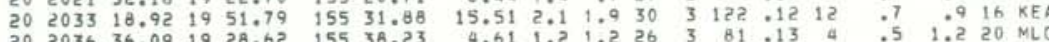

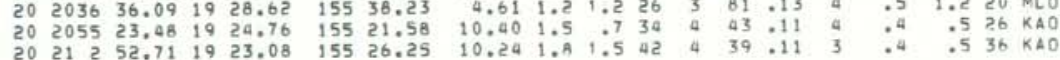

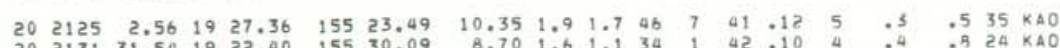

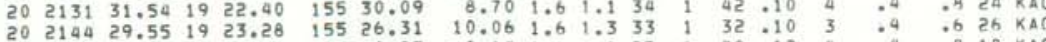

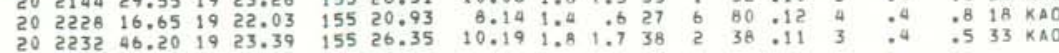

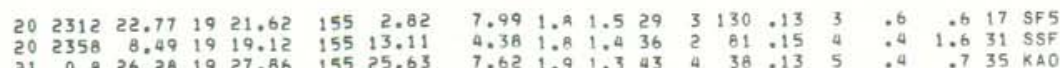

ORIGIN TIME LATN LON N OEPTH AMP NIR N GAP RMS MIN ERH ERZ NO

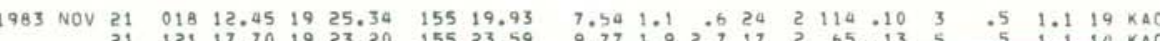

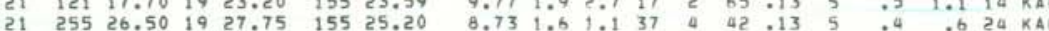

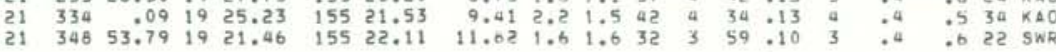
$\begin{array}{llllllllllllllllllll}21 & 911 & 33.94 & 19 & 24.78 & 155 & 26.47 & 8.34 & 2.0 & 1.5 & 34 & 1 & 36 & 10 & 5 & .40 & .4 & .9 & 30 & K A O \\ 21 & 440 & 48.15 & 19 & 28.08 & 155 & 26.45 & 8.84 & 1.9 & 1.5 & 36 & 3 & 48 & .12 & 6 & : 4 & .9 & 29 & K \Delta 0\end{array}$

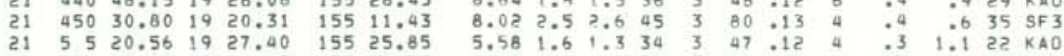
$\begin{array}{lllllllll}-1.15 & 1.3 & 1.5 & 29 & 1 & 163 & -10 & 3\end{array}$ 653 23.50 19 25.0815521 .25 8. 155 $.6 \quad 1.83155 \%$

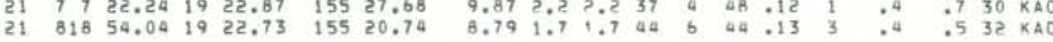

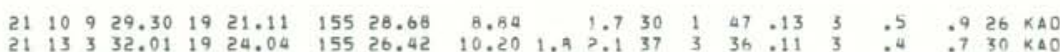

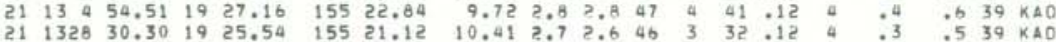

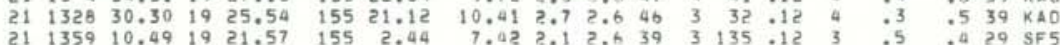
$\begin{array}{llllllllllllllllllllll}21 & 14 & 2 & 8.73 & 19 & 27.48 & 155 & 26.88 & 5.18 & 1.5 & 1.1 & 30 & 5 & 65 & .13 & 5 & .3 & 1.4 & 24 & K A C \\ 21 & 1448 & 45.74 & 19 & 22.15 & 155 & 29.93 & 8.83 & 2.6 & 3.1 & 46 & 2 & 33 & .11 & 4 & .3 & .6 & 42 & K A O\end{array}$

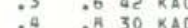

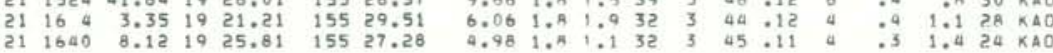

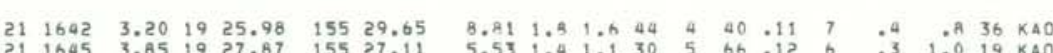

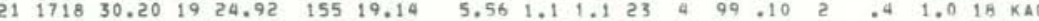

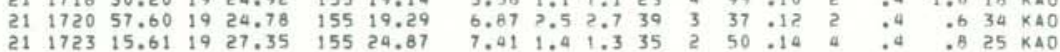

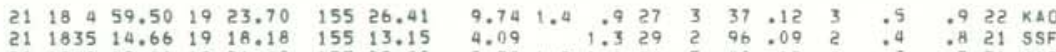

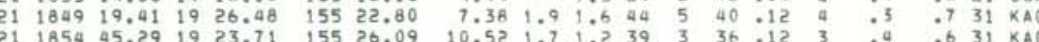

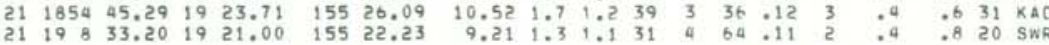
$\begin{array}{lllllllllllllllllll}21 & 1928 & 7.98 & 19 & 27.17 & 155 & 26.48 & 6.51 & 2.3 & 2.2 & 46 & 6 & 50 & .19 & 4 & .3 & .8 & 36 & \mathrm{KAO}\end{array}$

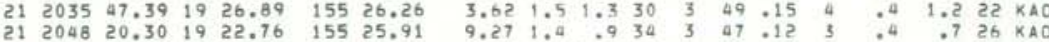

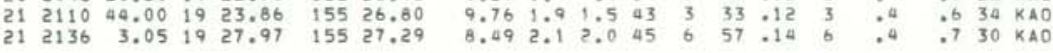

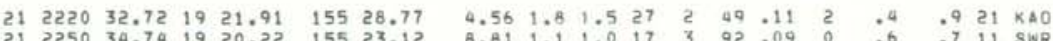

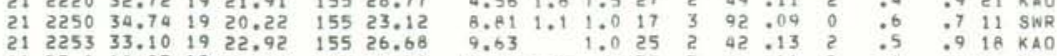

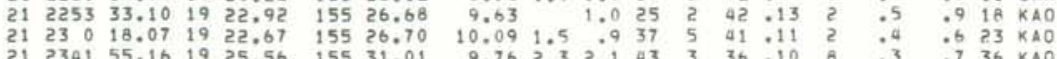

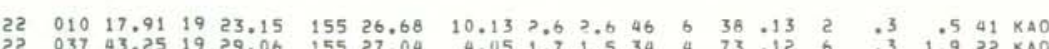

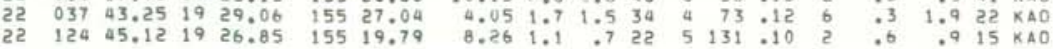


HVO EARTHQUAKE SUMMARY LIST

PAGE 77

ORIGIN TIME LAT N L LON W DEPTH AMP OUR NR GAP RMS MIN ERH ERZ ND
YEAR MON DA HRMN SEC OEG MIN OEG MIN KMM MAG MAG NR NS OEG SEC OIS KM KM FM REMK

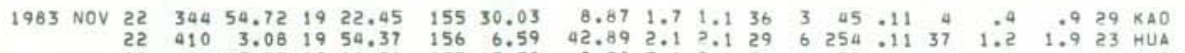

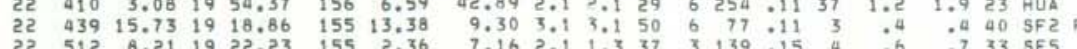

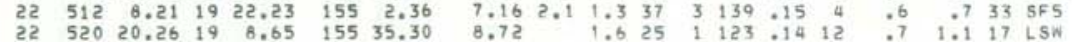
$\begin{array}{lllllllllllllllllll}22 & 550 & 39.01 & 19 & 26.21 & 155 & 23.62 & 7.48 & 2.0 & .4 & 41 & 2 & 40 & .13 & 3 & .9 & .9 & 34 & \text { KAO } \\ 22 & 553 & 11.34 & 19 & 56.49 & 155 & 31.11 & 34.18 & 3.2 & 3.0 & 52 & 6 & 159 & .12 & 19 & .7 & 1.7 & 45 & \text { KEA F }\end{array}$

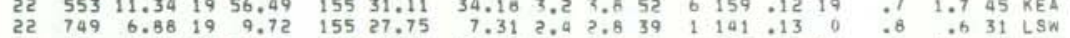

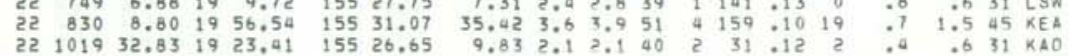

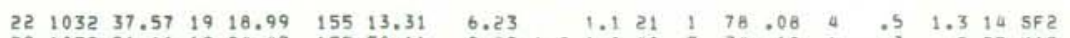

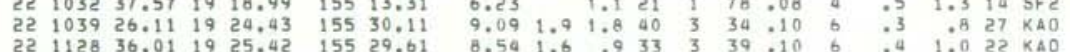

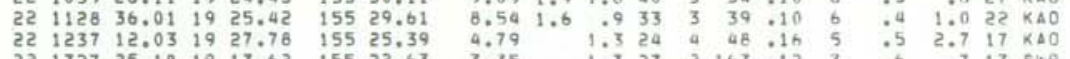

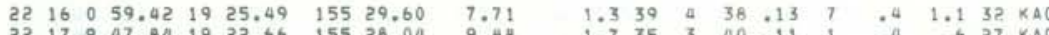

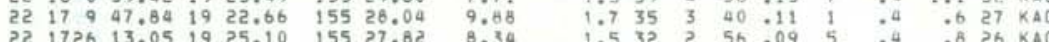

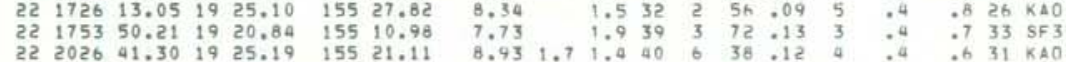

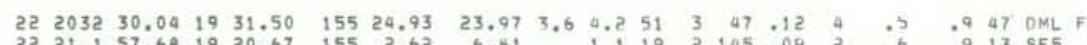

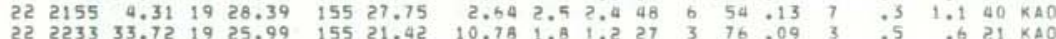

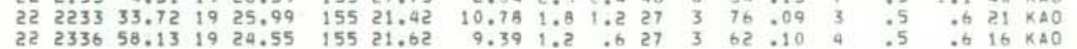

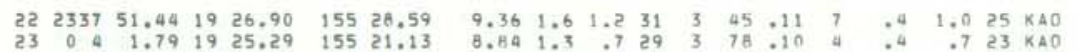

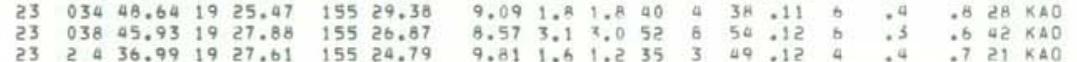

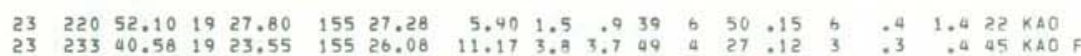

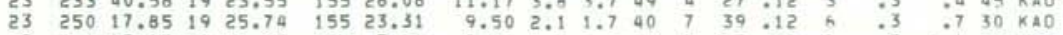

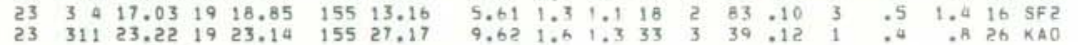

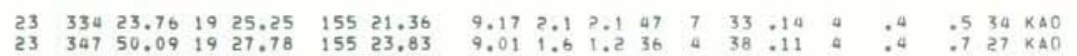

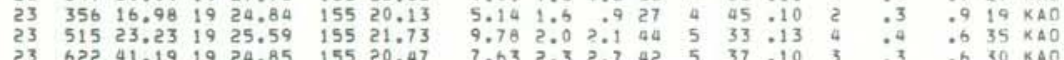

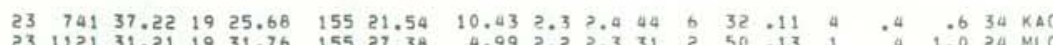

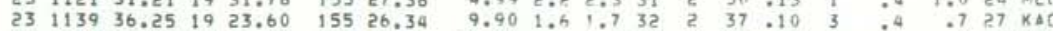

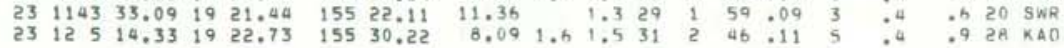

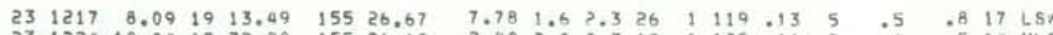

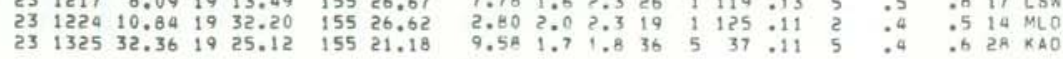

HVO EARTHQUAKE SUMMARY LIST

PAGE 7 B ORIGIN TIME LAT N LON W DEPTH AMP DIIR GAP RMS MIN ERH ERZ NO YEAR MON DA HRMN

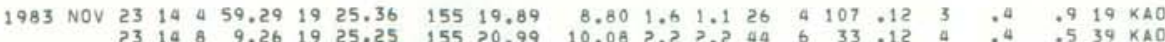

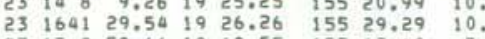

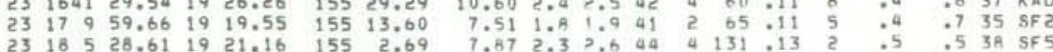

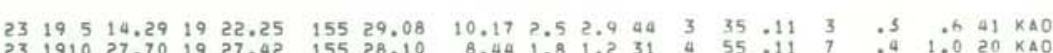

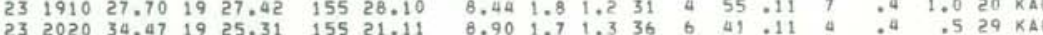

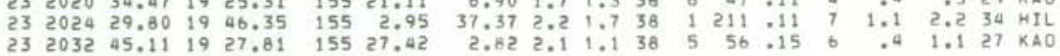

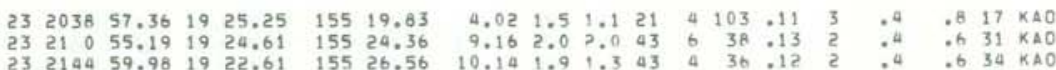

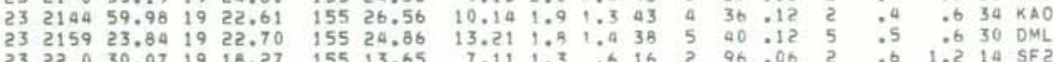

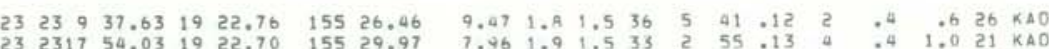

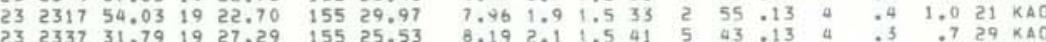

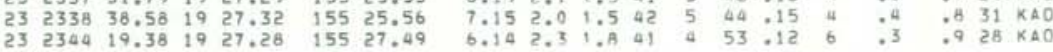

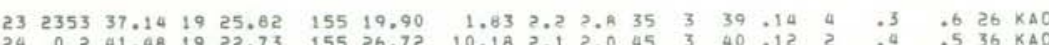

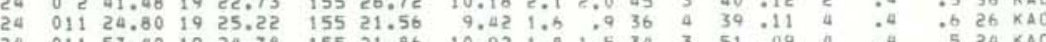

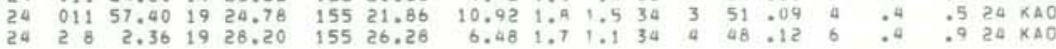

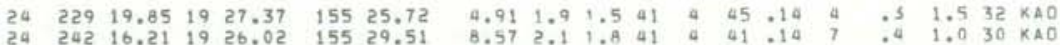

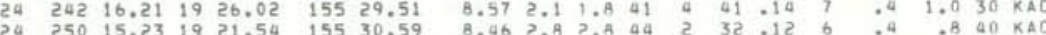

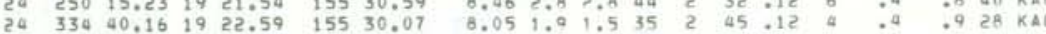

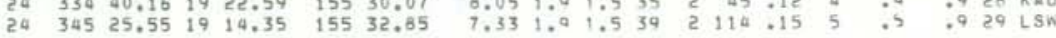

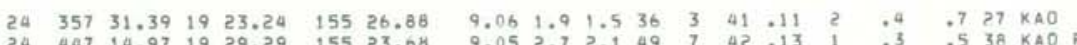

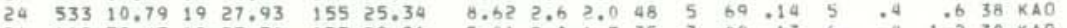

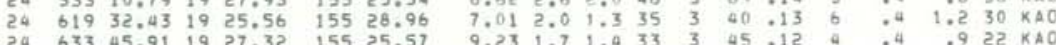
$24 \quad 740 \begin{array}{lllllllllllllllll}56.46 & 19 & 24.83 & 155 & 25.67 & 9.56 & 1.6 & 1.3 & 38 & 3 & 34 & .12 & 1 & .4 & .7 & 30 & K A O\end{array}$

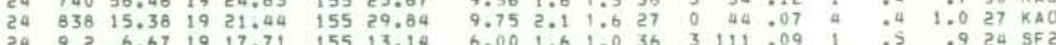

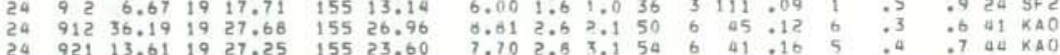
$\begin{array}{llllllllllllllllll}24 & 10 & 2 & 12.41 & 19 & 58.91 & 155 & 18.30 & 11.40 & 2.4 & 1.920 & 4 & 208 & .11 & 11 & 1.0 & .5 & 15 \\ \text { KEA }\end{array}$

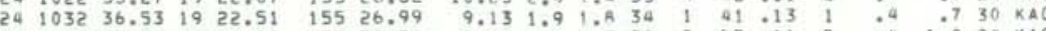

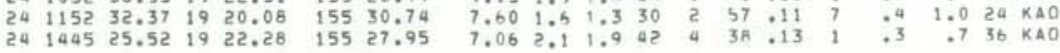
$\begin{array}{llllllllllllllllll}24 & 1456 & 15.63 & 19 & 27.28 & 155 & 28.44 & 8.85 & 1,8 & 1.0 & 32 & 2 & 54.13 & 7 & .4 & 1.0 & 19 & K A 0\end{array}$

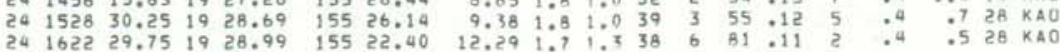


HVO EARTHQUAKE SUMMARY LIST

PAGE 79

ORIGIN TIME LATN LON N OEPTH AMP RIR G GAP RMS MIN ERH ERZ NO

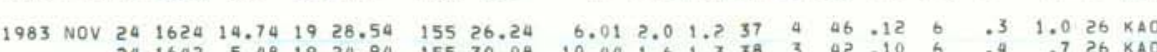

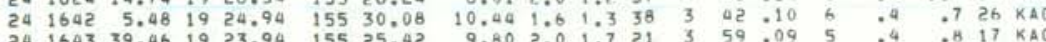

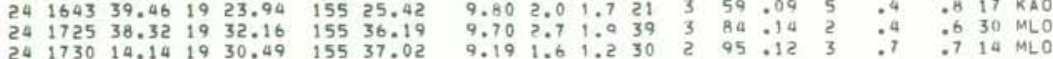

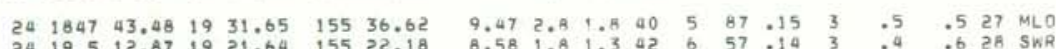

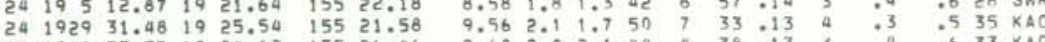

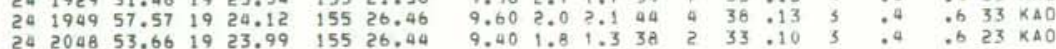

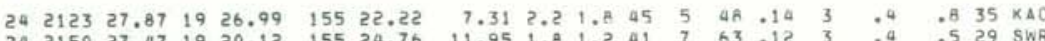

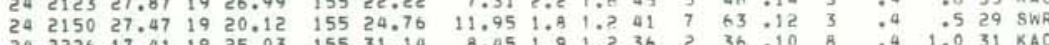

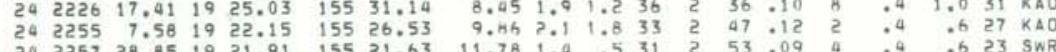

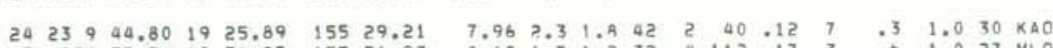

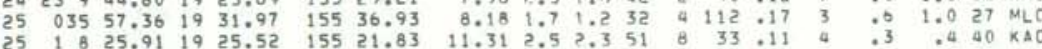

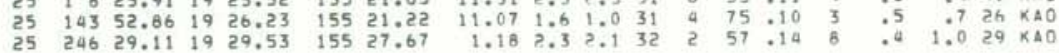

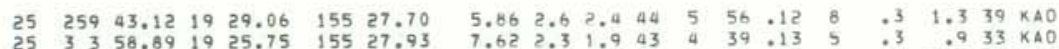

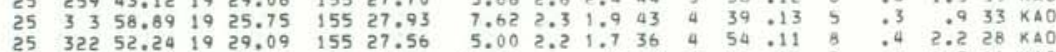

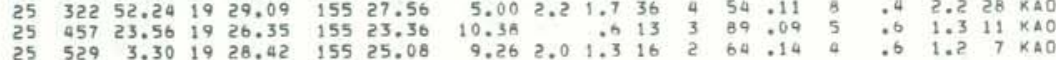

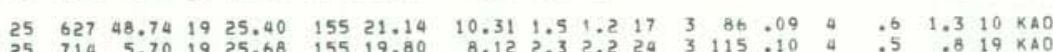

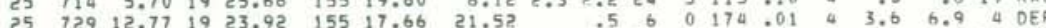

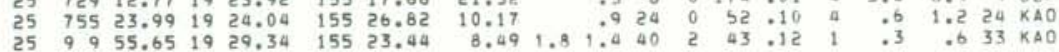

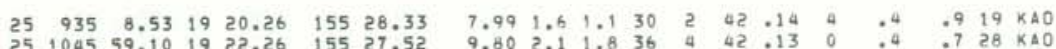

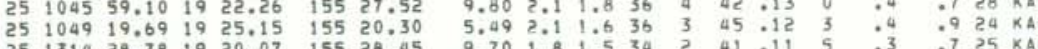

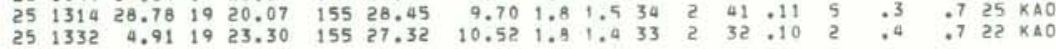
$\begin{array}{lllllllllllllllllll}25 & 1343 & 51.47 & 19 & 27.51 & 155 & 25.87 & 8.67 & 2.1 & 1.1 & 44 & 6 & 47 & 11 & 4 & .3 & .6 & 30 & \mathrm{KAO}\end{array}$

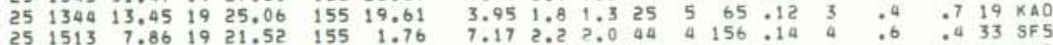

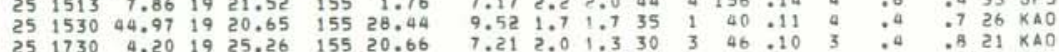

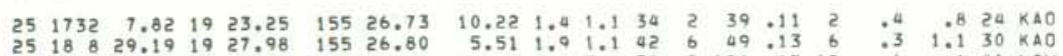

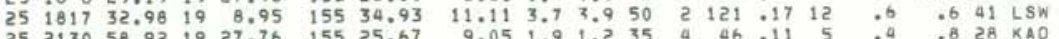

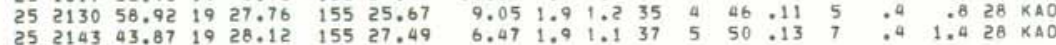

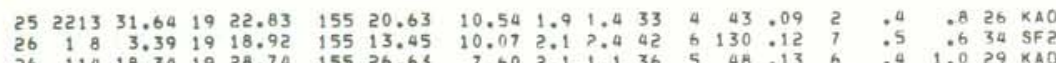

HVO EARTHQUAKE SUMMARY LIST

PAGE 80

ORIGIN TIME LAT N LON W DEPTH AMP DUR GAP RMS MIN ERH ERZ NO

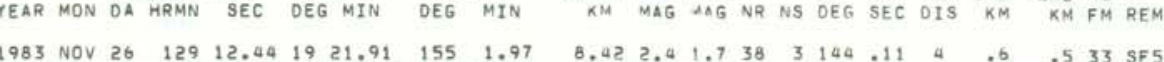

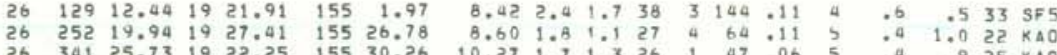

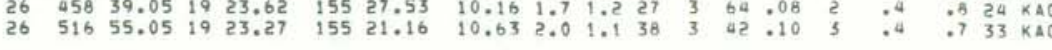

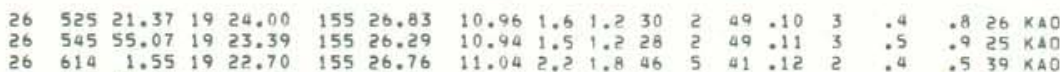

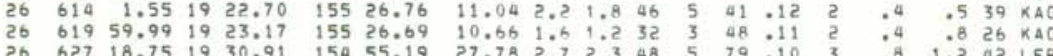

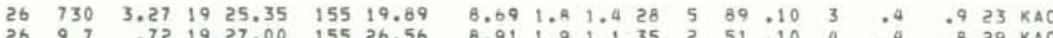

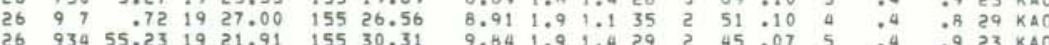

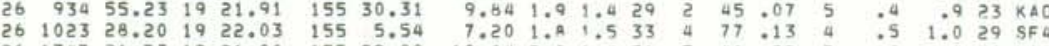

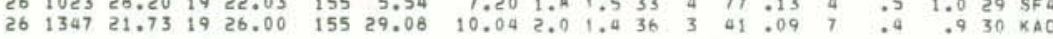

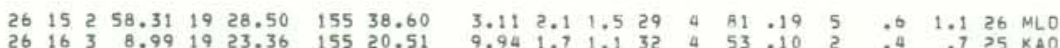

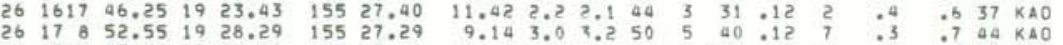

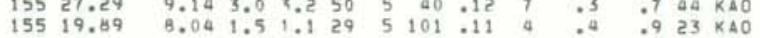

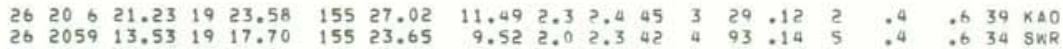

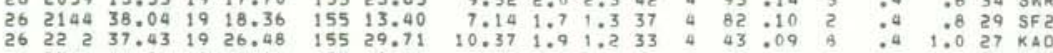

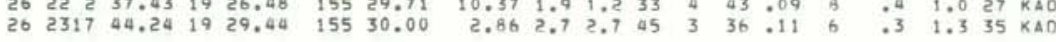

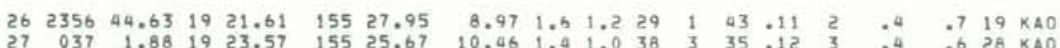

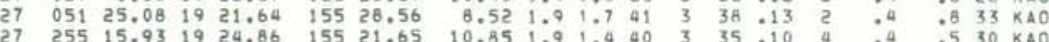

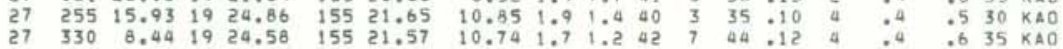

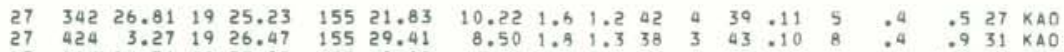

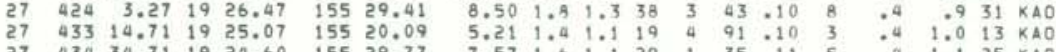

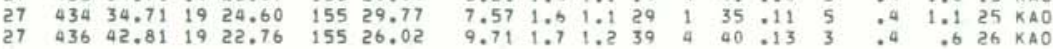

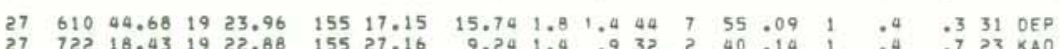

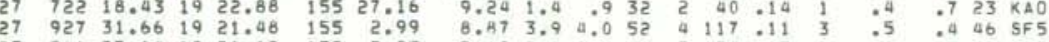

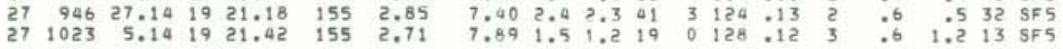

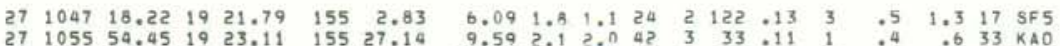

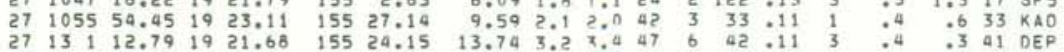

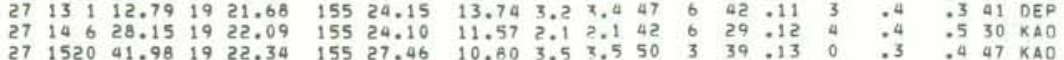

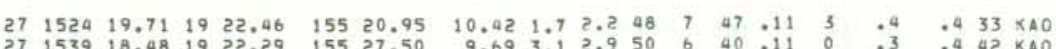

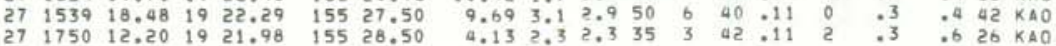




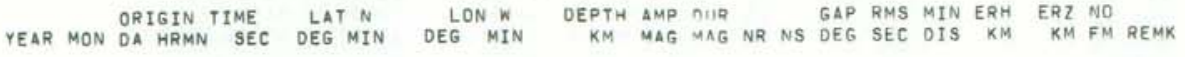

1983 Nov $27 \quad 19249.1019 \quad 19.91 \quad 15513.29$

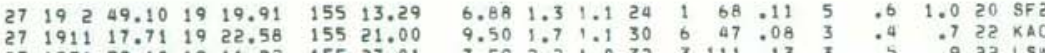

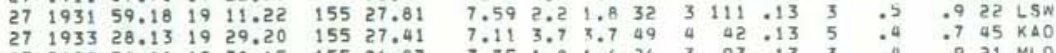

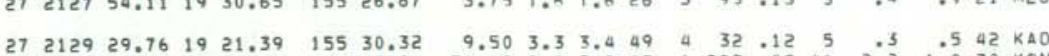

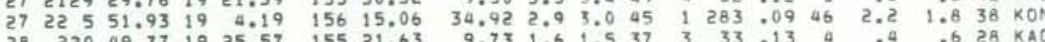

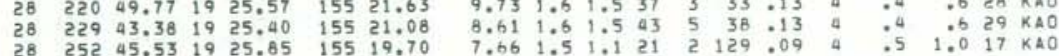

$\begin{array}{lllllllllllllllllll}28 & 314 & 4.78 & 19 & 23.74 & 155 & 26.27 & 10.72 & 2.4 & 2.5 & 45 & 3 & 26 & .13 & 3 & .4 & .6 & 37 & K A O\end{array}$

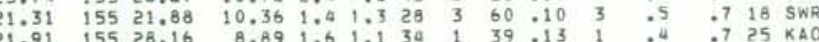

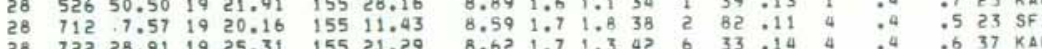

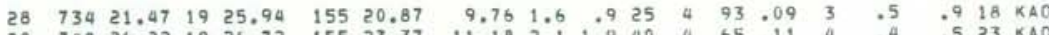

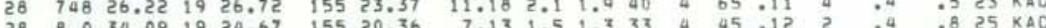

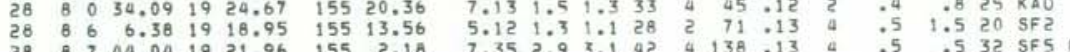

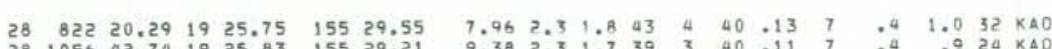

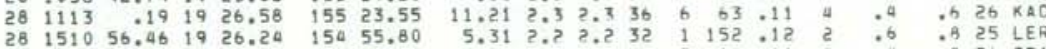

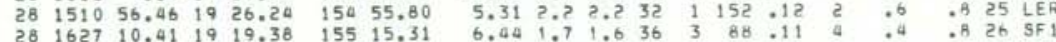

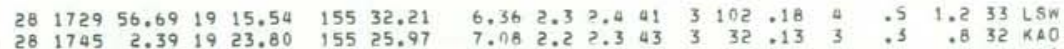

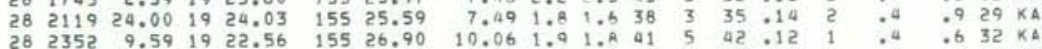

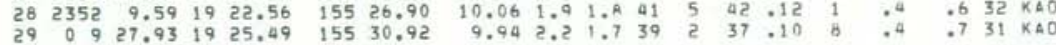
$\begin{array}{lllllllrlllllllllllll}29 & 151 & 58.01 & 19 & 17.47 & 155 & 12.94 & 5.81 & 1.8 & .8 & 24 & 2 & 138 & .12 & 1 & .6 & 1.1 & 16 & 5 F 2 \\ 29 & 233 & 4.76 & 19 & 24.87 & 155 & 21.59 & 10.45 & 1.7 & 7.7 & 39 & 5 & 36 & .11 & 4 & .4 & .5 & 27 & \mathrm{KAD}\end{array}$

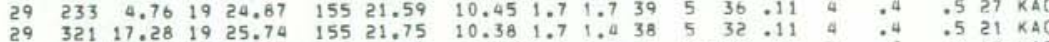

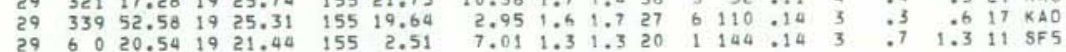
\begin{tabular}{lllllllllllllllllll}
29 & 830 & 26.30 & 19 & 25.85 & 155 & 27.50 & 7.21 & 2.2 & 1.9 & 34 & 1 & 39 & .12 & 4 & .4 & 1.1 & 33 & $K A 0$ \\
\hline
\end{tabular}

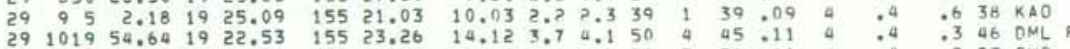

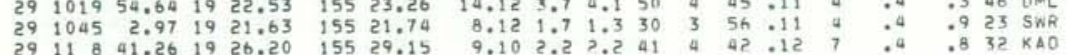

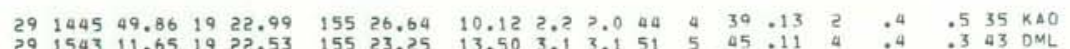

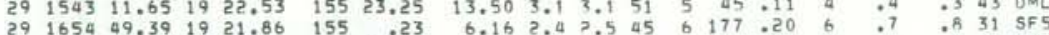

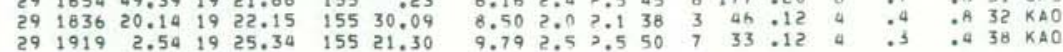
$\begin{array}{llllllllllllllllllll}29 & 2131 & 51.32 & 19 & 23.88 & 155 & 27.03 & 10.11 & 2.0 & 2.1 & 42 & 3 & 36 & .12 & 3 & .4 & .6 & 32 & K A 0\end{array}$

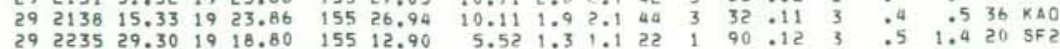

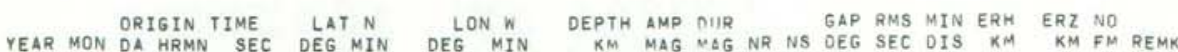
1983 NOV $30 \quad 152 \quad 6.411927 .03 \quad 15525603$

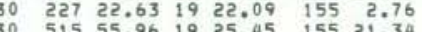

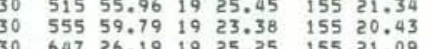
$30 \quad 155950.84 \quad 1926.98 \quad 15523.64$ $30 \quad 163844.79$ 19 $20.21 \quad 15512.64$

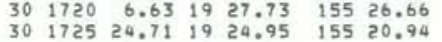
$\begin{array}{llllllll}30 & 20 & 6 & 58.43 & 19 & 23.18 & 155 & 25.47\end{array}$ DEC $1 \quad 013 \quad 25.83 \quad 1921.83 \quad 15521.51$

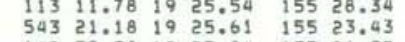

$\begin{array}{lllll}71 & .65 \quad 19 & 22.42 \quad 155 & 29.96\end{array}$

$\begin{array}{llllll}716 & 31.96 & 19 & 23.99 & 155 & 26.94 \\ 733 & 47.36 & 19 & 25.86 & 155 & 24.13\end{array}$

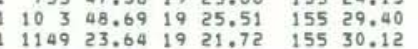

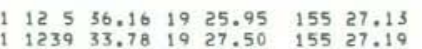

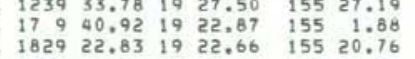

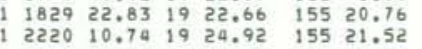

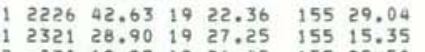

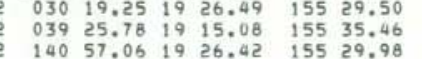

$\begin{array}{lllllll}2 & 256 & 4.54 & 19 & 21.43 & 155 & 2.67\end{array}$

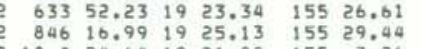

$\begin{array}{lllll}1014 & 52.33 \quad 19 & 20.23 \quad 155 & 30.17\end{array}$

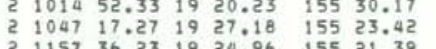

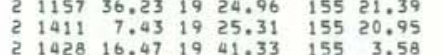

$\begin{array}{lllllll}2 & 1532 & 8.19 & 19 & 22.54 & 155 & 20.79 \\ 2 & 1620 & 23.91 & 19 & 23.83 & 155 & 16.88\end{array}$

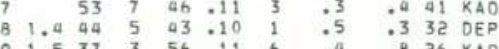

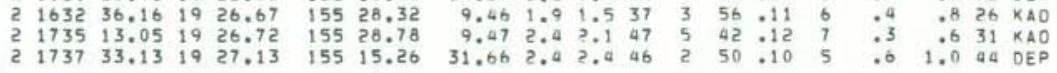
$\begin{array}{llllllllllllllllllll}2 & 1839 & 6.13 & 19 & 25.42 & 155 & 29.60 & 7.11 & 2.2 & 1.5 & 39 & 3 & 38 & 13 & 6 & .9 & 1.1 & 27 & K A O \\ 2 & 1926 & 7.10 & 19 & 28.67 & 155 & 23.99 & 9.97 & 1.8 & 1.2 & 36 & 2 & 68 & .13 & 2 & .5 & .7 & 27 & K A O\end{array}$ 
HVO EARTHQUAKE SUMMARY LIST

PAGE Aै

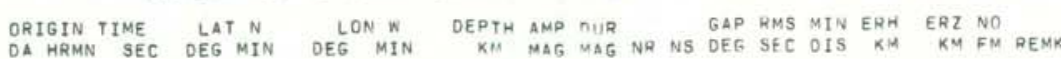
YEAR MON DA HRMN SECC DEG MIN OEG MIN OEM MAG MAG NA NS DEG SEC OIS KM EQZ NE DEMK

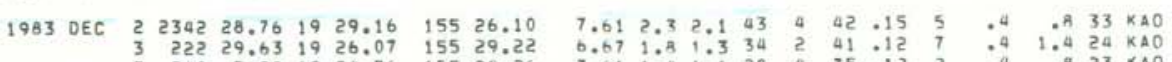

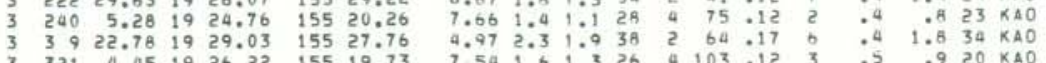

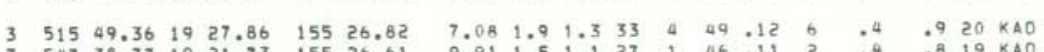

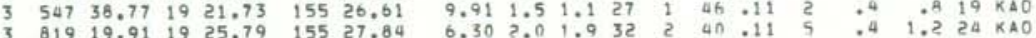

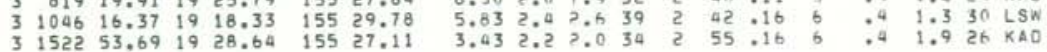

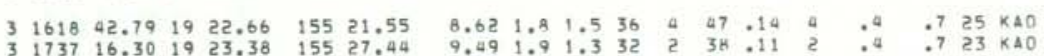

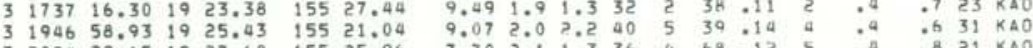

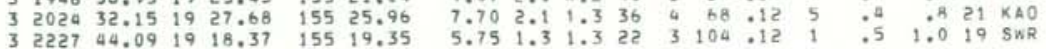

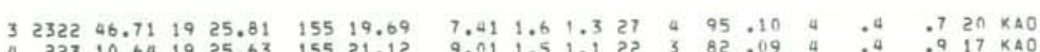

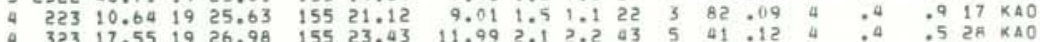

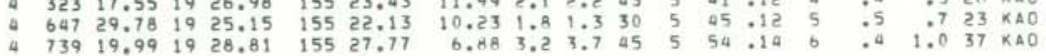

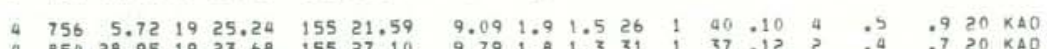

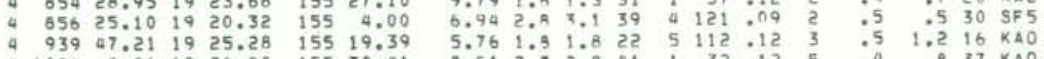

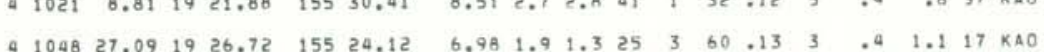

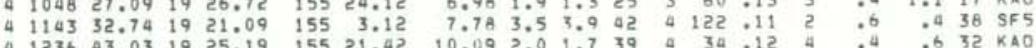

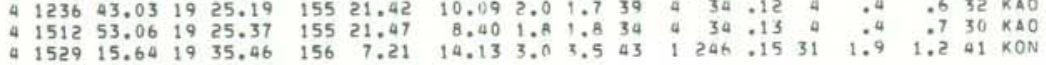

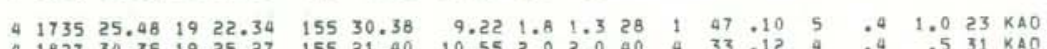

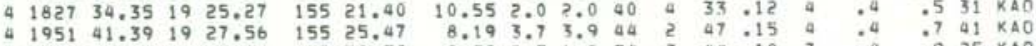

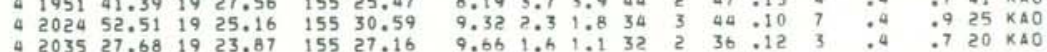

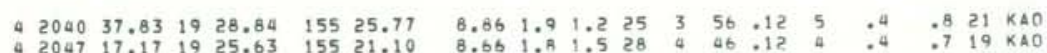

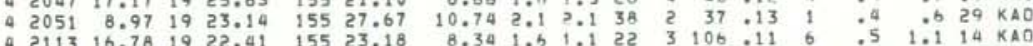

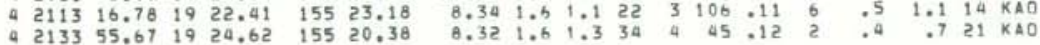

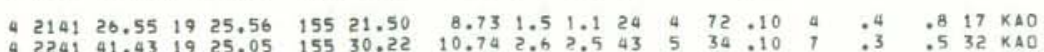

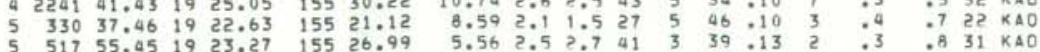

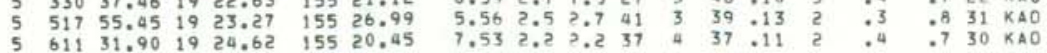

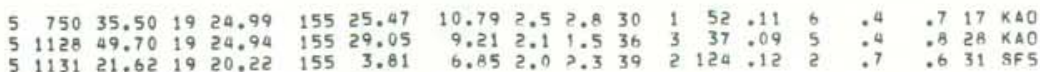

HVO EARTHQUARE SUMMARY LIST

PAGE AS

ORIGIN TIME
DA HRMN SAT N

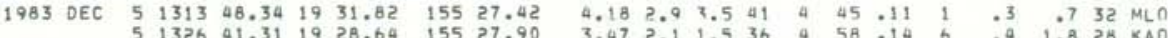

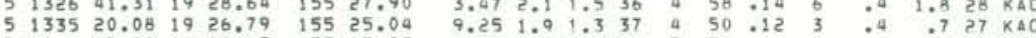

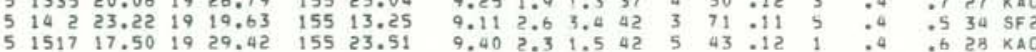
$\begin{array}{lllllllllllllllllll}5 & 1817 & 30.50 & 19 & 22.12 & 155 & 17.15 & 31.24 & 2.4 & 1.9 & 44 & 4 & 44 & .12 & 2 & .7 & .9 & 35 & \text { DEP }\end{array}$

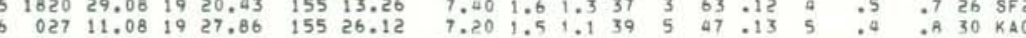

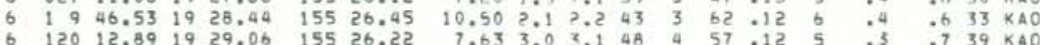

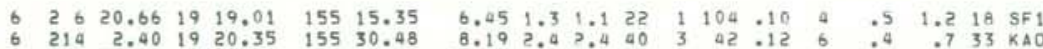

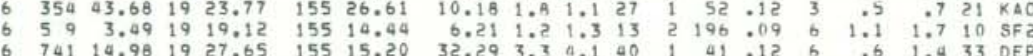

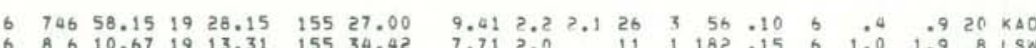

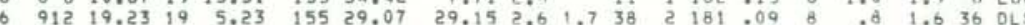

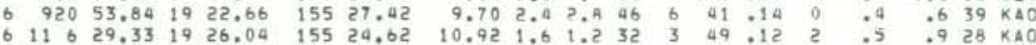

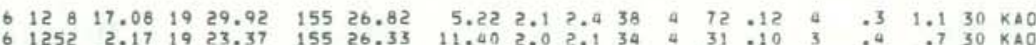

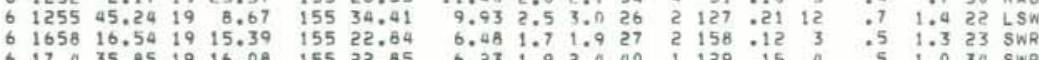

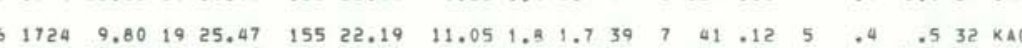

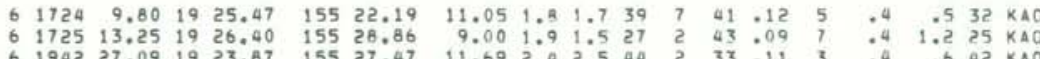

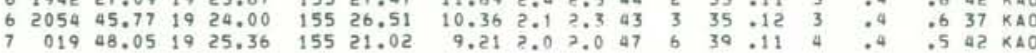

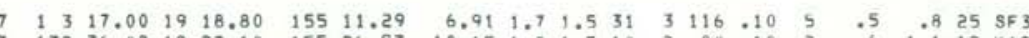

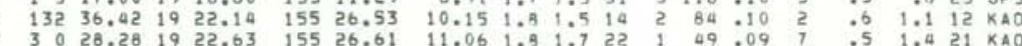

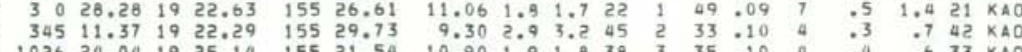

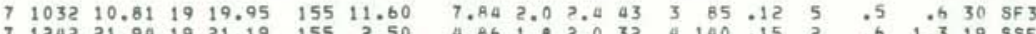

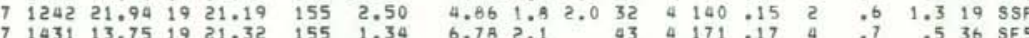

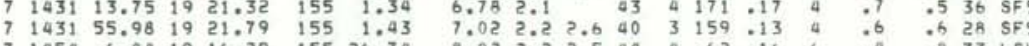

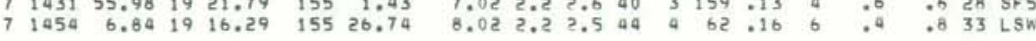
$\begin{array}{lllllllllllllllllll}7 & 1744 & 13.18 & 19 & 23.14 & 155 & 27.23 & 9.69 & 1.9 & 1.3 & 38 & 3 & 43 & .13 & 1 & .4 & .6 & 23 & \mathrm{kAO}\end{array}$

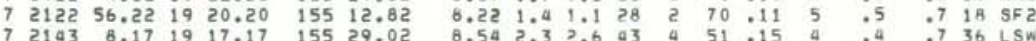

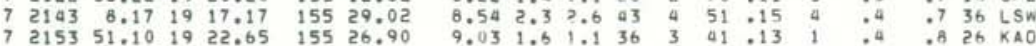

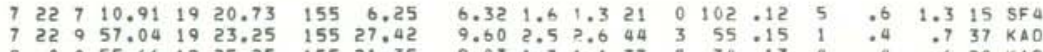


1983 DEC $8 \begin{array}{lllllllllllllllllll}235 & 17.28 & 19 & 22.96 & 155 & 27.69 & 10.30 & 2.6 & 2.7 & 95 & 5 & 35 & .12 & 1 & .4 & .6 & 38 & K A O\end{array}$

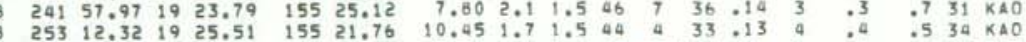

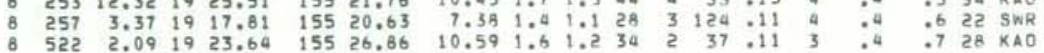

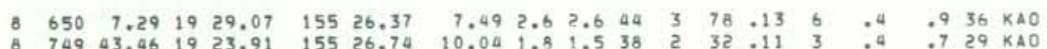

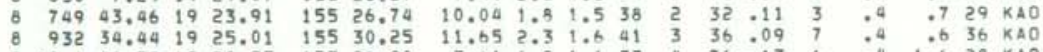

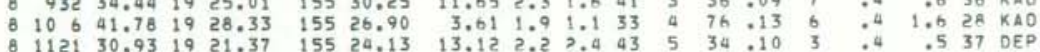
$\begin{array}{lllllllllllllllllll}8 & 1419 & 45.82 & 19 & 25.07 & 155 & 21.69 & 11.46 & 2.8 & 3.2 & 54 & 10 & 35 & .11 & 4 & .3 & .3 & 97 & K A O\end{array}$

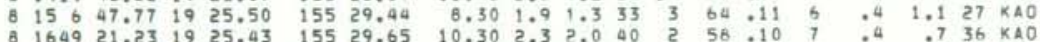

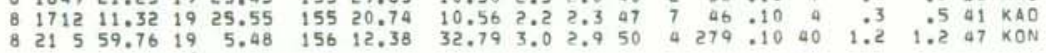

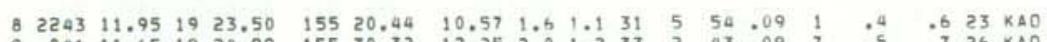

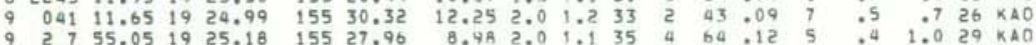

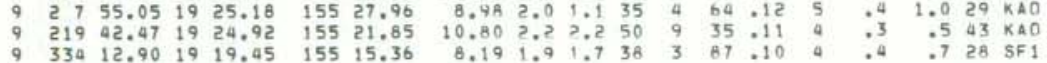

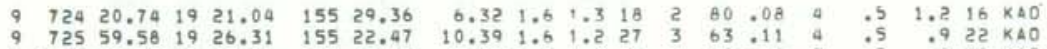
$9 \begin{aligned} & 1023 \\ & 93.30 \\ & 9\end{aligned}$

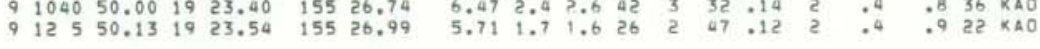

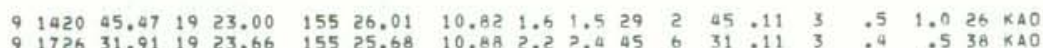

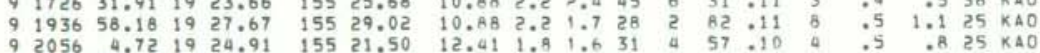

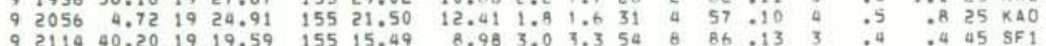

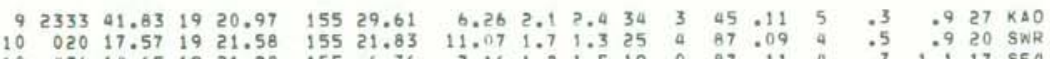

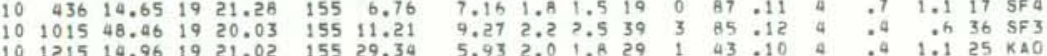
$\begin{array}{lllllllllllllllllll}10 & 1230 & 31.24 & 19 & 20.97 & 155 & 23.61 & 10.47 & 2.2 & 2.5 & 40 & 4 & 35 & .11 & 2 & .4 & .5 & 29 & \text { SWR } \\ 10 & 324 & 54.09 & 19 & 2.10 & 155 & 24.24 & 12.73 & 2.4 & 2.4 & 53 & 8 & 27 & -11 & 4 & .3 & .3 & 39 & \text { KAO }\end{array}$

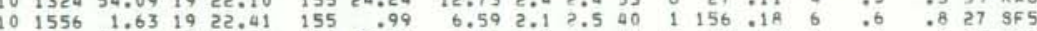

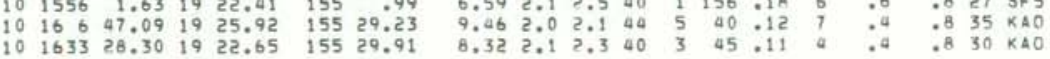

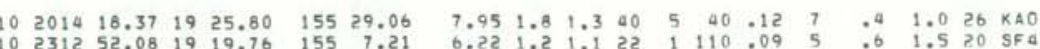

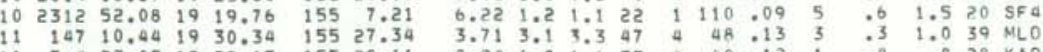

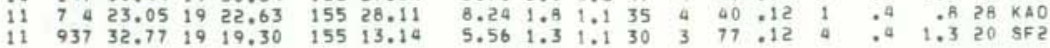

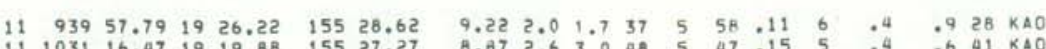

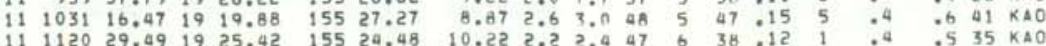

HVO EARTHQUAKE SUMMARY LIST

PAGE B6

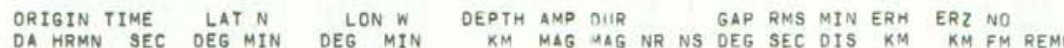

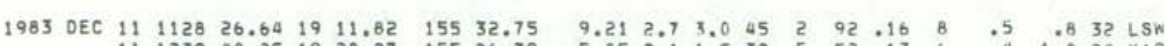

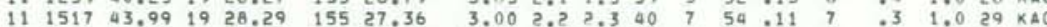

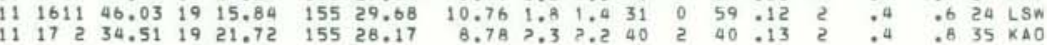

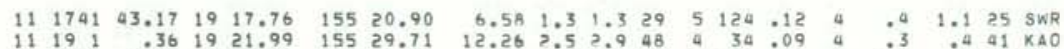

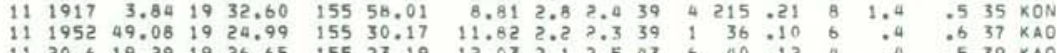
$\begin{array}{lllllllllllllllllllll}11 & 2152 & 53.01 & 19 & 20.23 & 155 & 12.97 & 6.98 & 1.4 & 1.3 & 34 & 3 & 68 & .15 & 4 & .5 & .8 & 29 & \text { SF } & \end{array}$

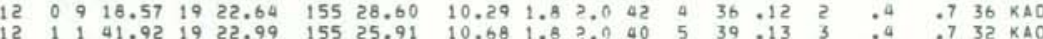

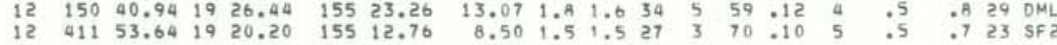

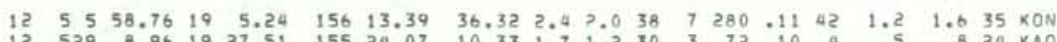

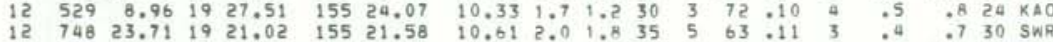

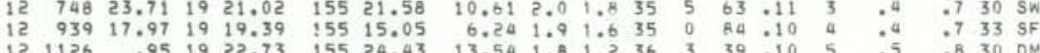
\begin{tabular}{rlllllllllllllllllll}
12 & 1251 & 57.96 & 19 & 24.02 & 155 & 26.92 & 10.66 & 2.0 & 2.4 & 43 & 4 & 33 & .12 & 3 & .4 & .6 & 37 & KAO \\
12 & 1422 & 20.04 & 19 & 17.58 & 155 & 21.16 & 8.01 & 1.0 & 1.3 & 29 & 3 & 125 & 010 & 5 & $: 4$ & 1.1 & 21 & SWR \\
\hline
\end{tabular}

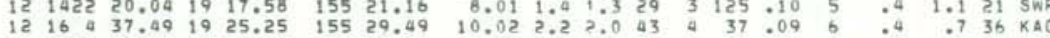

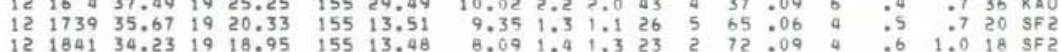

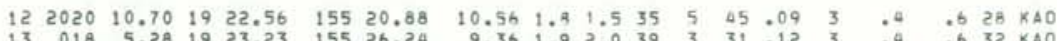

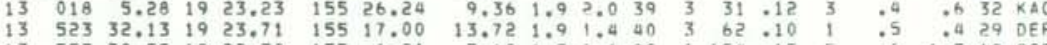

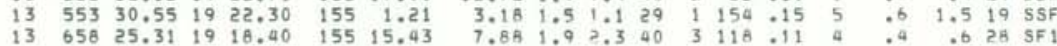

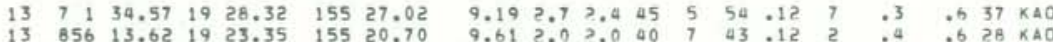

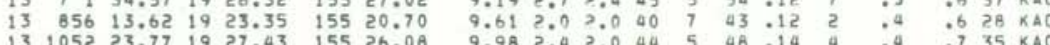

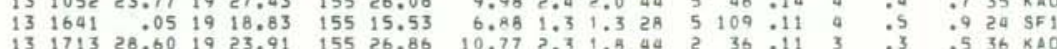

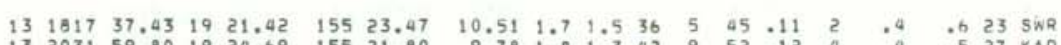

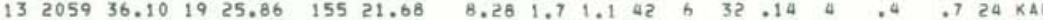

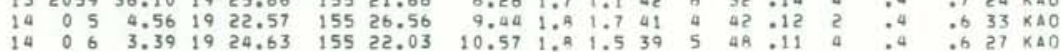
$\begin{array}{lllllllllllllllllllll}14 & 225 & 18.28 & 19 & 20.50 & 155 & 29.89 & 6.08 & 2.0 & 1.8 & 34 & 3 & 48 & 11 & 5 & .4 & 1.1 & 27 & \times 40 \\ 14 & 42 & 48.17 & 19 & 29.29 & 155 & 47.63 & 8.55 & 2.3 & 1.8 & 29 & 4 & 79 & : 14 & 4 & : 6 & .7 & 20 & \times 0 N\end{array}$

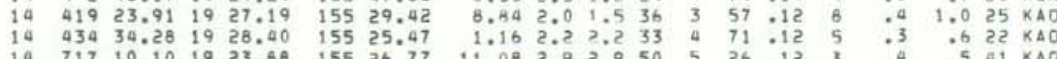

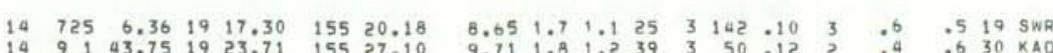

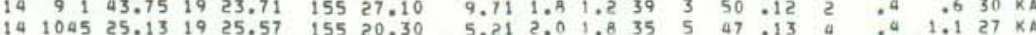


HVO EARTHQUAKE SUMMARY LIST

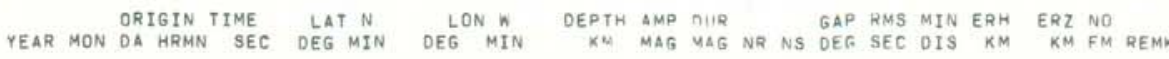

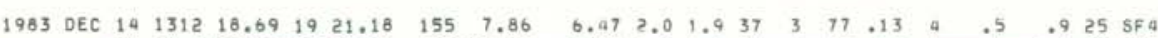

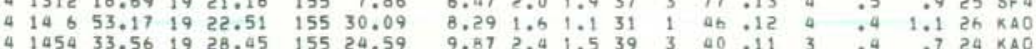

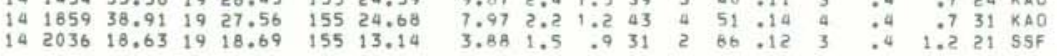

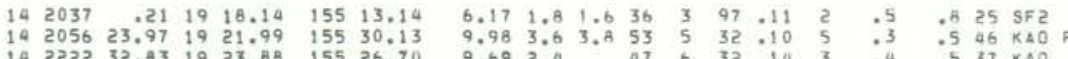

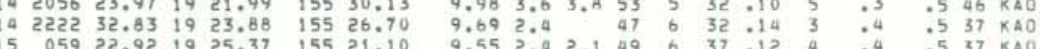

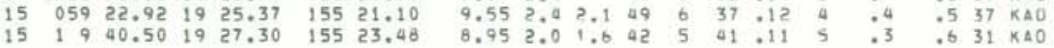

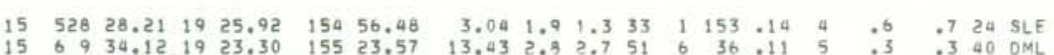

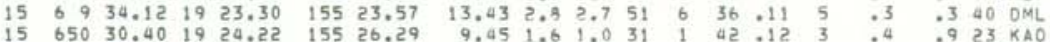

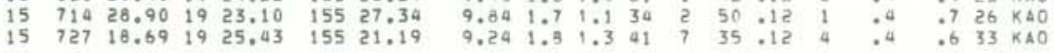

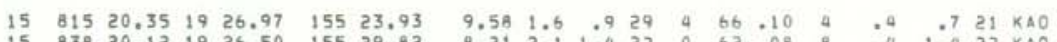

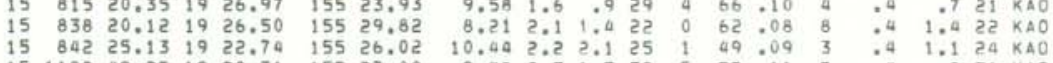

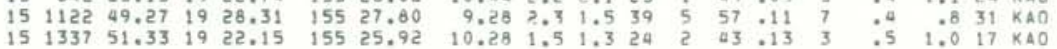

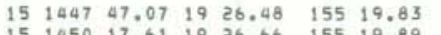

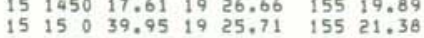

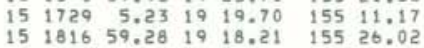

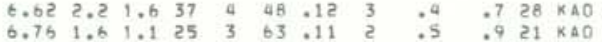

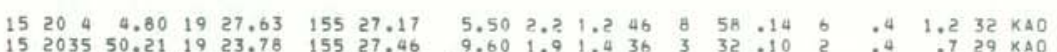

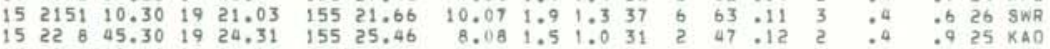

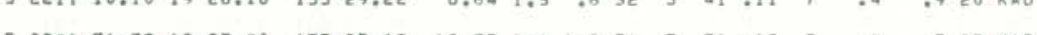

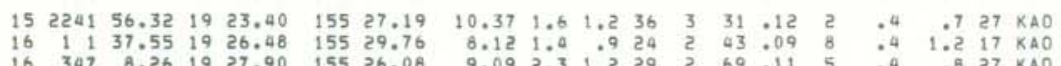

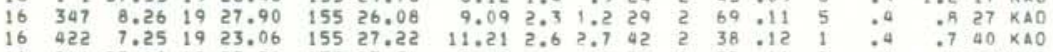

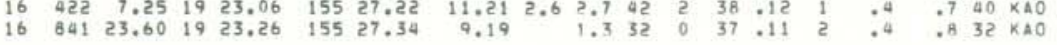

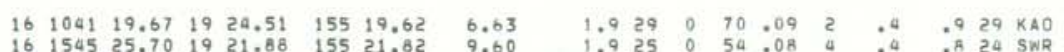

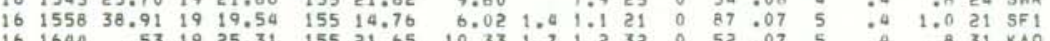

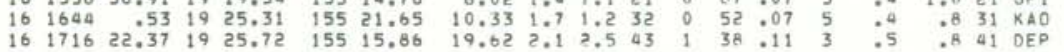
$\begin{array}{lllllllllllllllllllll}16 & 2031 & 2.52 & 19 & 22.78 & 155 & 20.76 & 8.78 & 2.0 & 1.5 & 33 & 0 & 47 & .11 & 3 & .4 & .6 & 33 & \text { KAO } \\ 16 & 2047 & 3.43 & 19 & 19.52 & 155 & 15.65 & 6.34 & 2.1 & 1.3 & 29 & 0 & 90 & -10 & 3 & .4 & .6 & 29 & 5 F 10\end{array}$

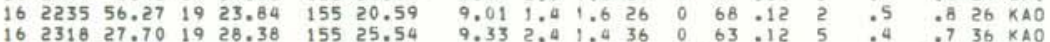

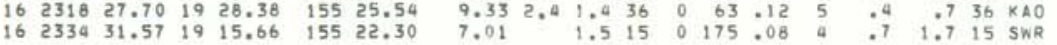

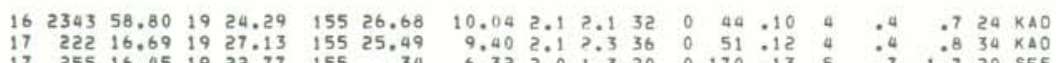

HVO EARTHQUAKE SUMMARY LIST

PAGE BQ

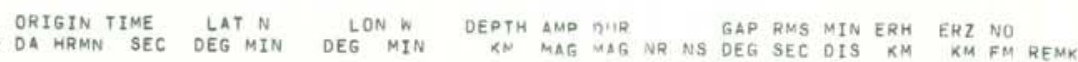

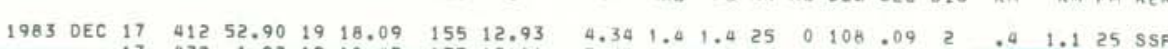

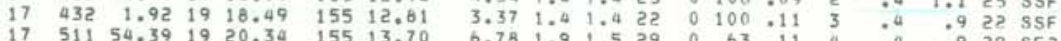

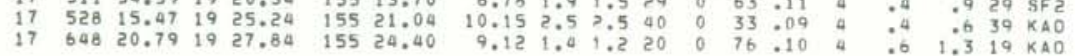

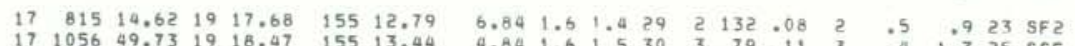

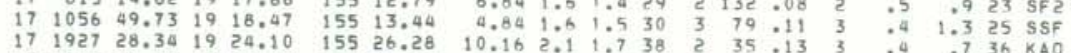

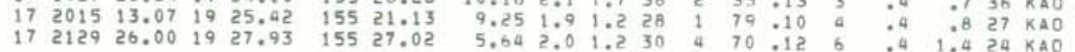

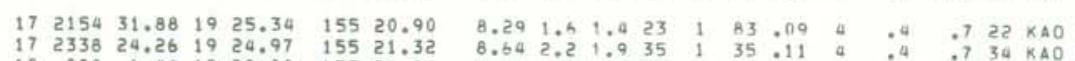

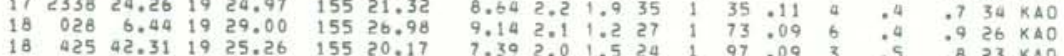

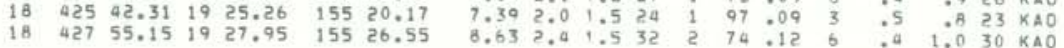

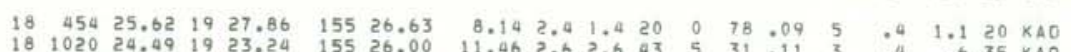

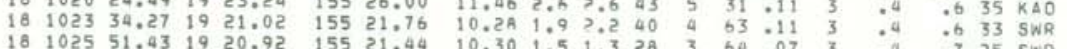

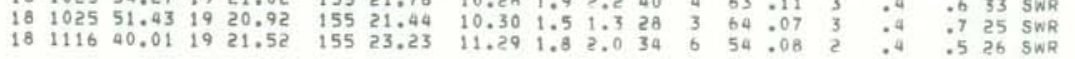

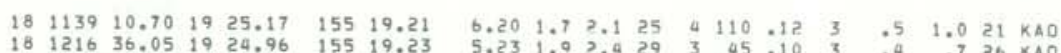

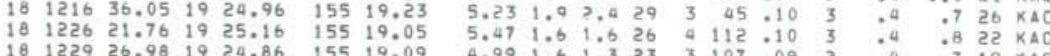

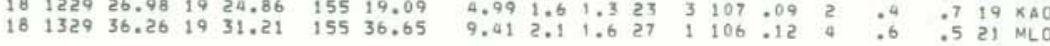

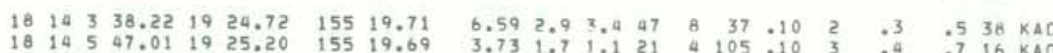
$\begin{array}{llllllllllllllll}18 & 1411 & 9.15 & 19 & 25.14 & 155 & 19.44 & 3.58 & 1.4 & 1.3 & 26 & 5 & 106 & 12 & 3 & 4\end{array}$

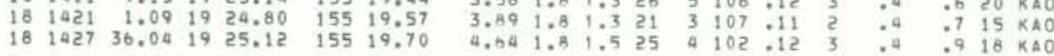

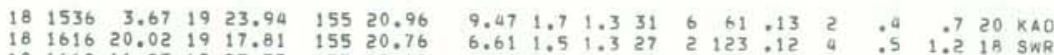

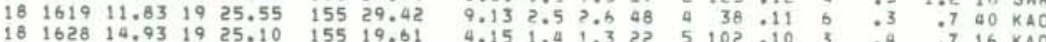

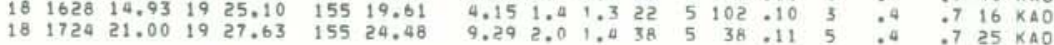

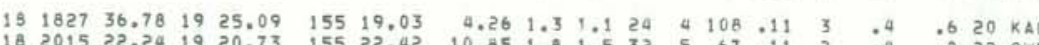

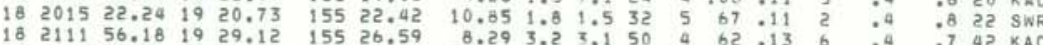

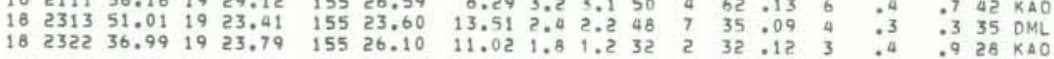

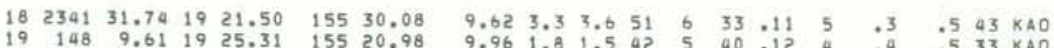

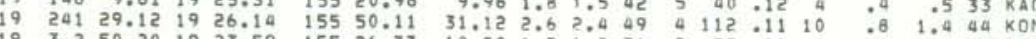

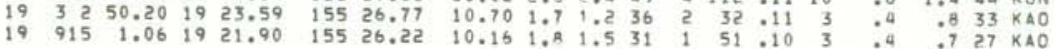

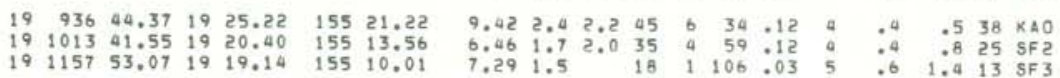


ORIGIN TIME LAT N
YEAR MON DA HRMN SEC DEG MIN DEG MIN OEPTH AMP OUR NR GAP RMS MIN ERH ERZ NO
KAG MAG NR NS DEG SEC DIS KM KM FM REMK

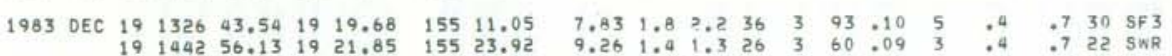

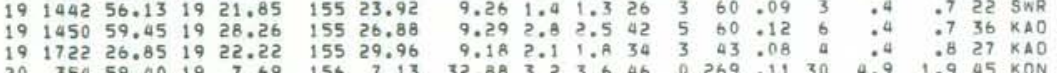

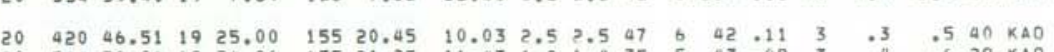

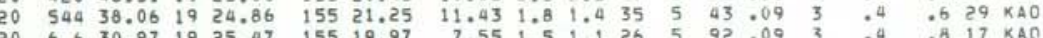

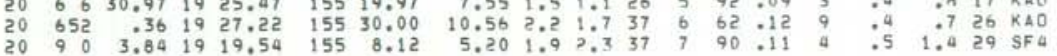

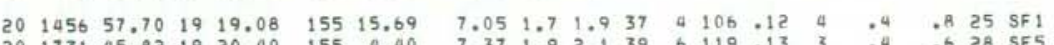

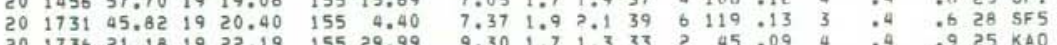

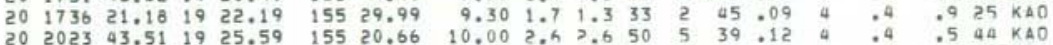

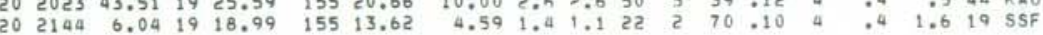

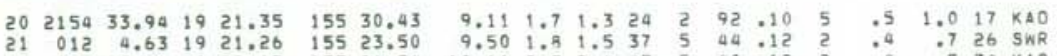

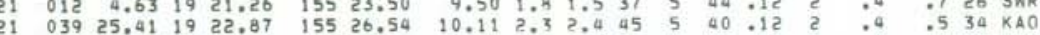

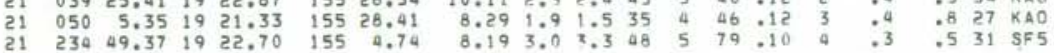

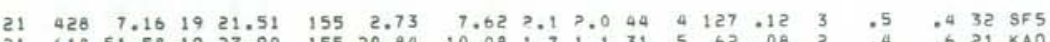

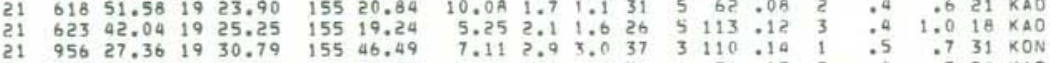

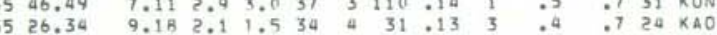

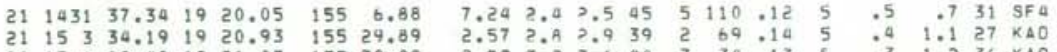

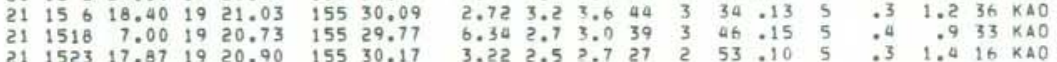

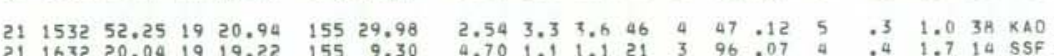

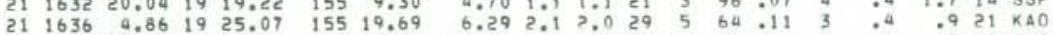

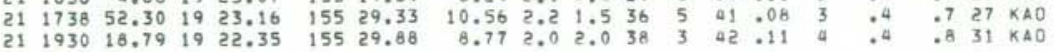

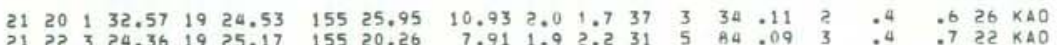

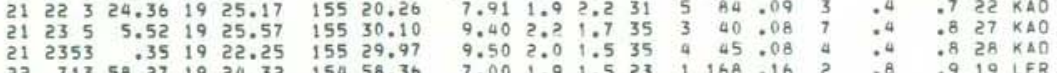

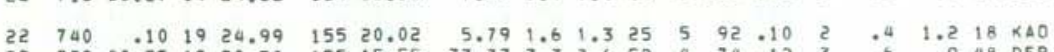

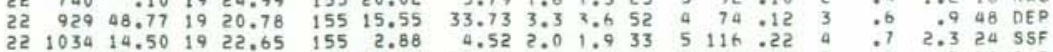

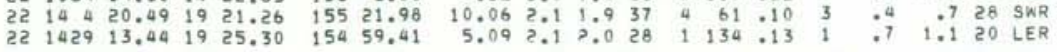

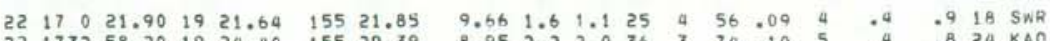

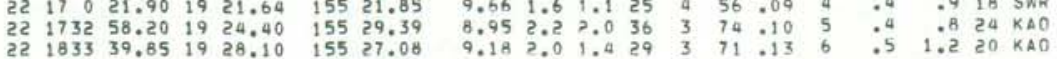

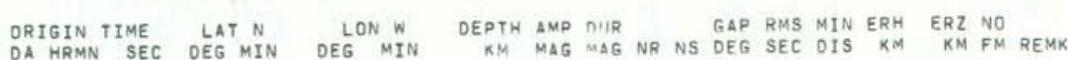

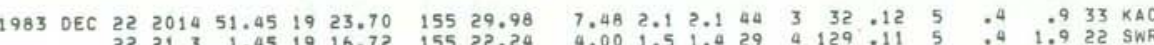

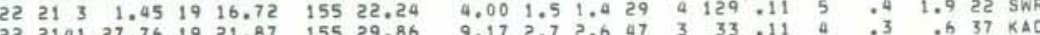

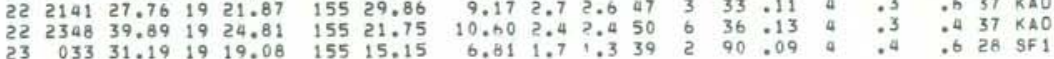

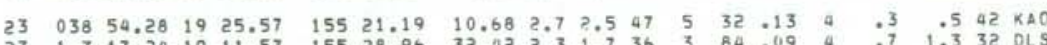

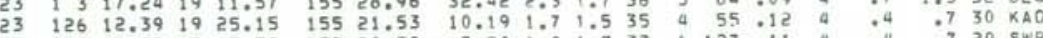

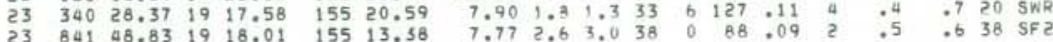

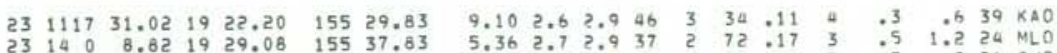

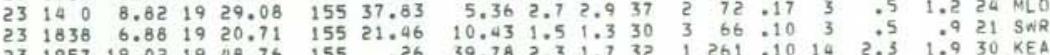

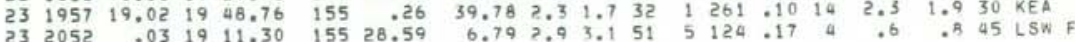

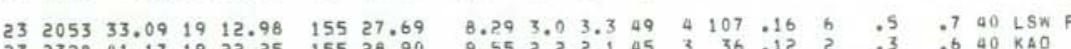

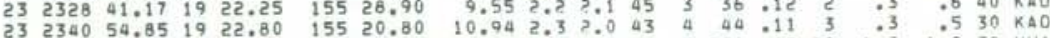

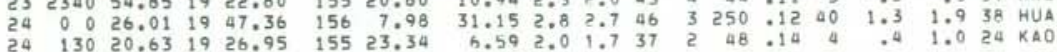

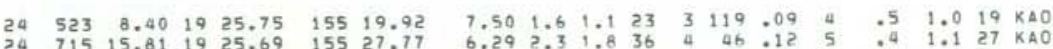

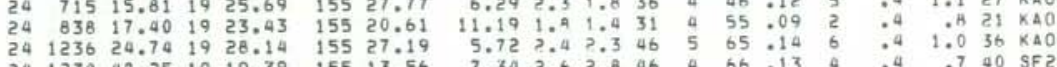

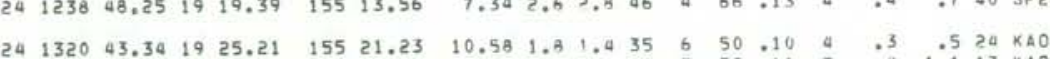

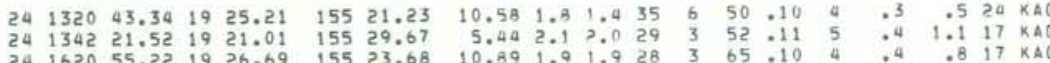

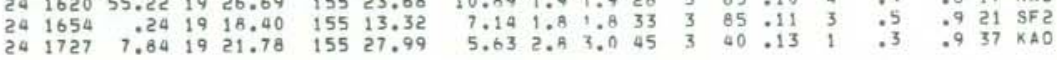

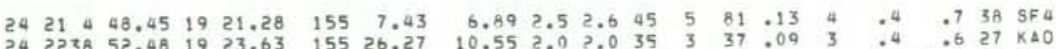

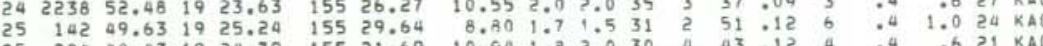

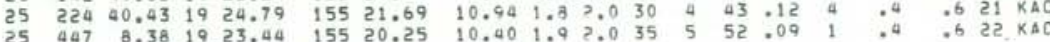

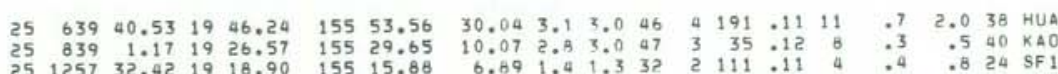

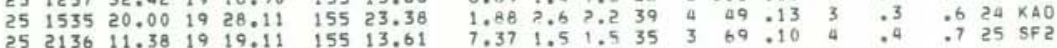
$\begin{array}{lllllllllllllllllll}26 & 214 & 52.11 & 19 & 20.98 & 155 & 2.77 & 4.80 & 1.9 & 2.1 & 34 & 5 & 130 & .17 & 2 & .5 & 1.3 & 18 & 55 F\end{array}$

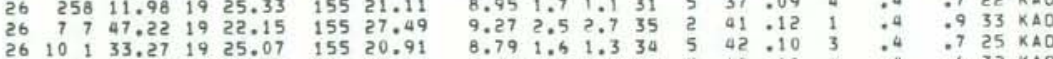

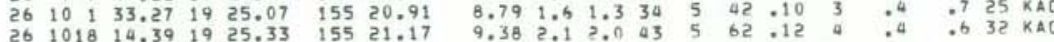

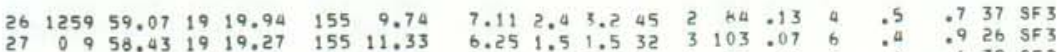




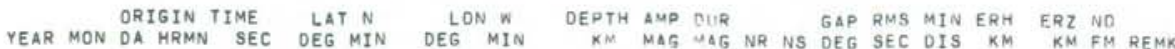

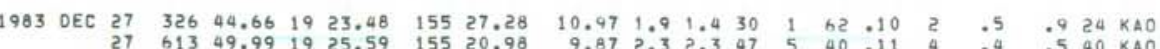

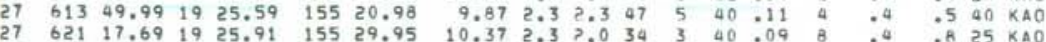

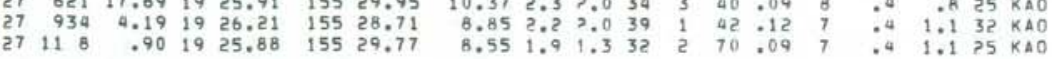

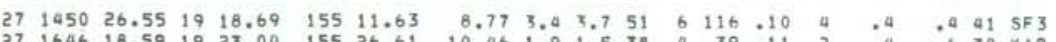

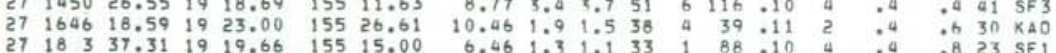

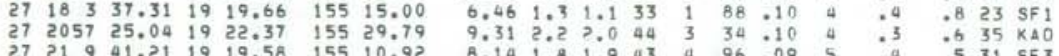

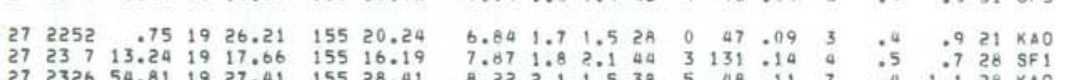

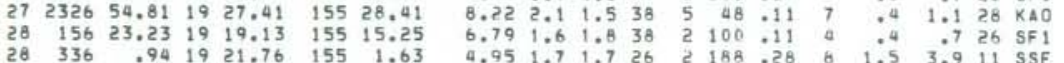

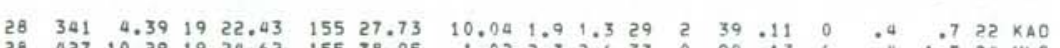

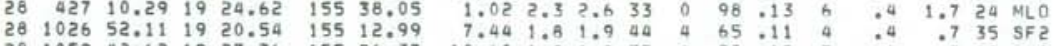

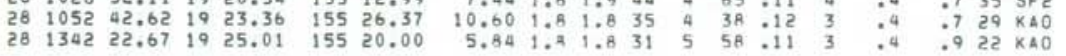

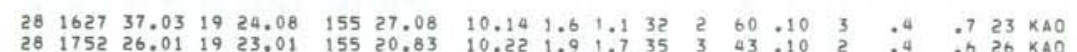

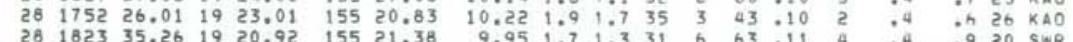

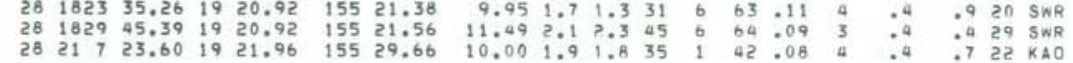

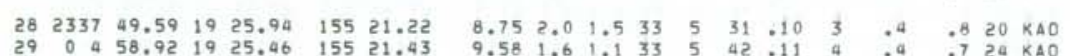

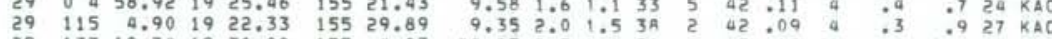

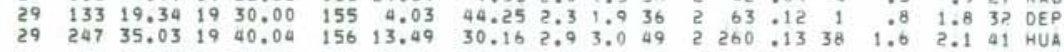

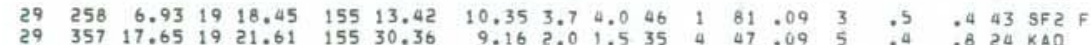

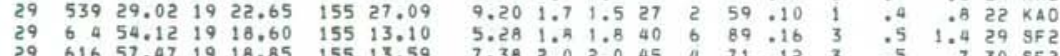
$\begin{array}{llllllllllllllllllll}29 & 620 & 57.19 & 19 & 19.84 & 155 & 12.41 & 6.19 & 1.7 & 1.3 & 29 & 2 & 80 & .11 & 5 & .5 & 1.1 & 23 & 5 F 2\end{array}$

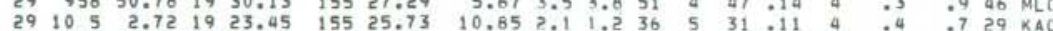

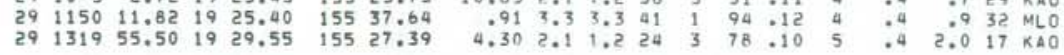

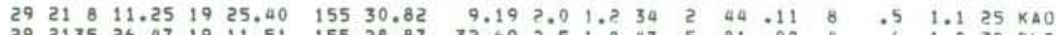

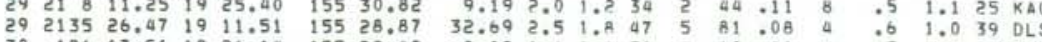

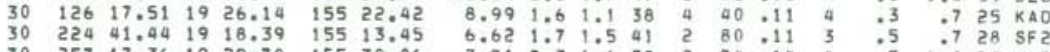

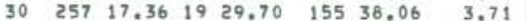

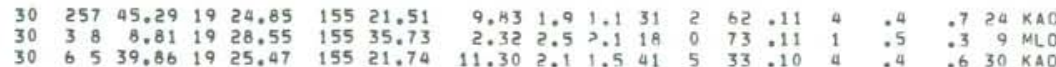

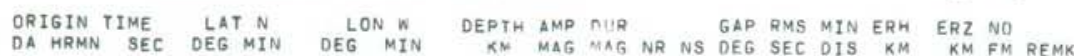

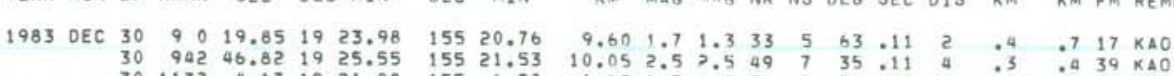

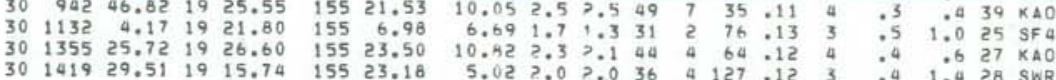
$\begin{array}{llllllllllllllllllll}30 & 1535 & 27.21 & 19 & 18.37 & 155 & 13.33 & 5.23 & 1.5 & 1.5 & 27 & 3 & 85 & .10 & 3 & .4 & 1.1 & 21 & \text { SF } & \end{array}$

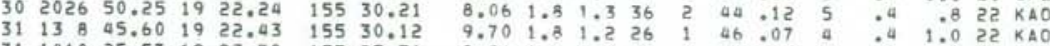

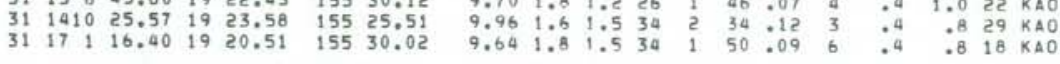
$\begin{array}{lllllllllllllllllll}31 & 2127 & 50.06 & 19 & 16.56 & 155 & 22.83 & 5.87 & 1.9 & 9.9 & 36 & 1 & 121 & .15 & 5 & .5 & 1.2 & 24 & \text { SWR } \\ 31 & 2243 & 14.83 & 19 & 26.36 & 155 & 37.53 & 1.42 & 2.5 & 2.2 & 23 & 1 & 93 & .14 & 3 & .04 & .7 & 14 & \text { MLO }\end{array}$ 
Table 6. HVO EARTHQUAKE SUMMARY LIST

PAGE 1

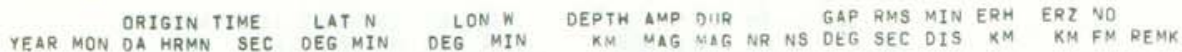

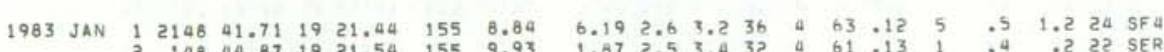

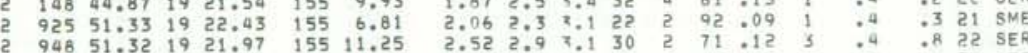

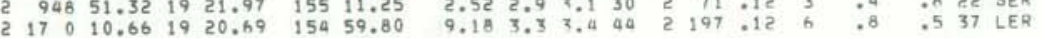

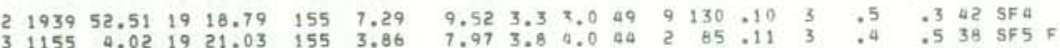

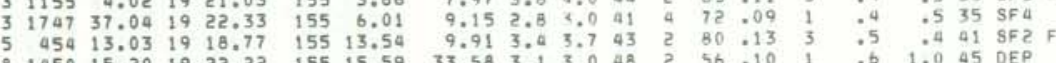

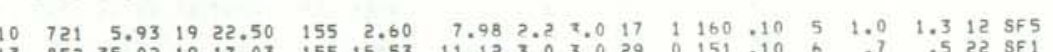

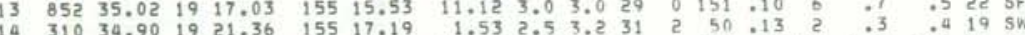

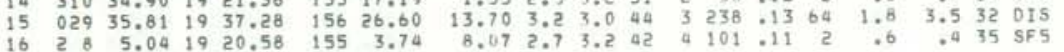

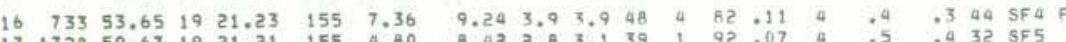

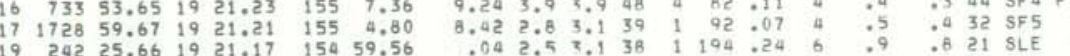

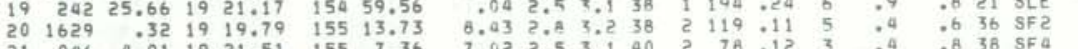

$\begin{array}{lllllll}21 & 122 & 36.12 & 19 & 25.65 & 155 & 37.83 \\ 21 & 859 & 42.36 & 19 & 20.91 & 154 & 59.03\end{array}$

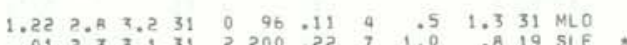

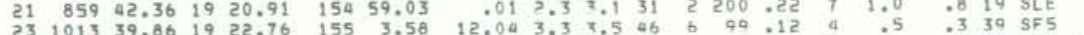

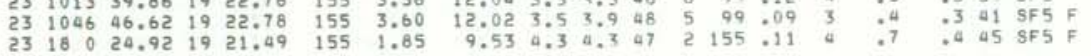

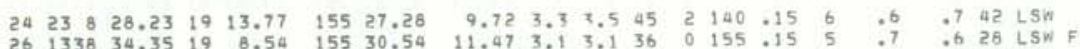

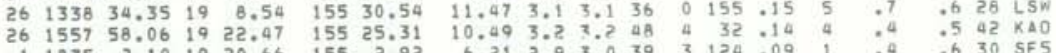

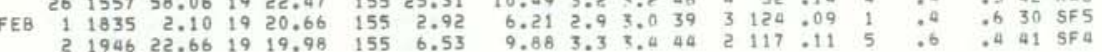

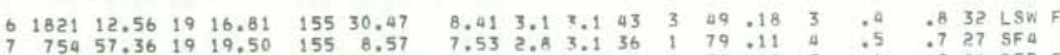

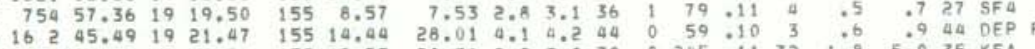

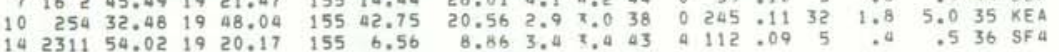

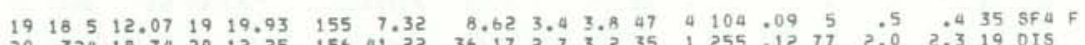

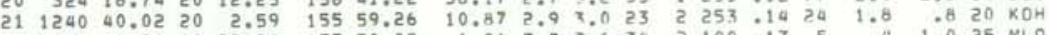

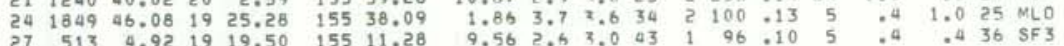

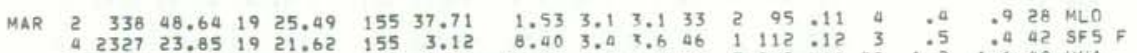

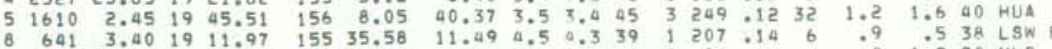

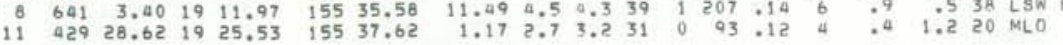

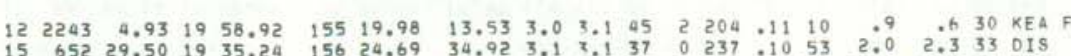

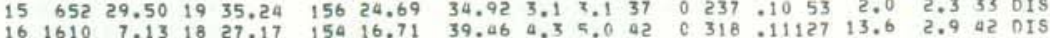

HVO EARTHQUAKE SUMMARY LIST

PAGE

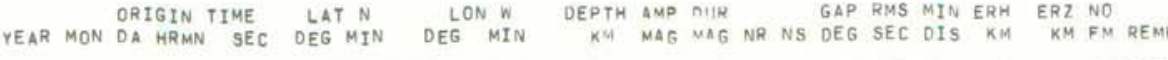

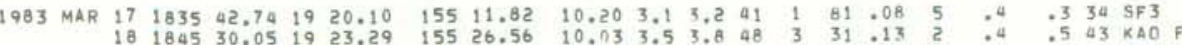

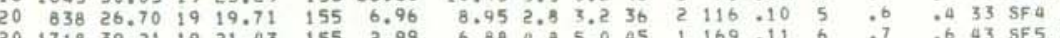

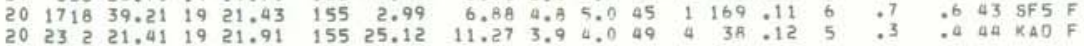

$\begin{array}{lllllllllllllllllll}21 & 037 & 28.88 & 19 & 22.01 & 155 & 25.42 & 11.32 & 3.4 & 3.6 & 46 & 3 & 39 & 13 & 4 & .4 & .4 & 41 & k A 0 \\ 21 & 2136 & 23.05 & 19 & 21.22 & 155 & 27.76 & 11.16 & 3.3 & 7.3 & 47 & 4 & 42 & 013 & 2 & .4 & \text {.5 } & 36 & k 40\end{array}$

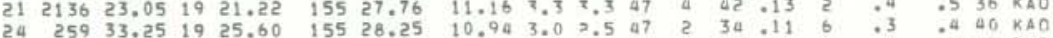

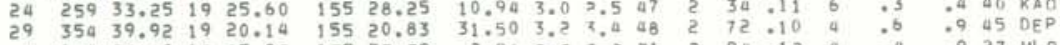

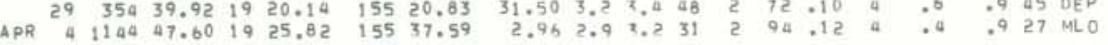

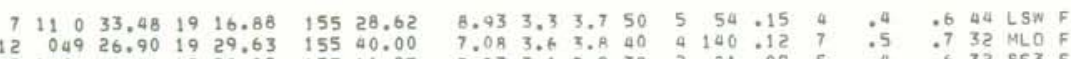

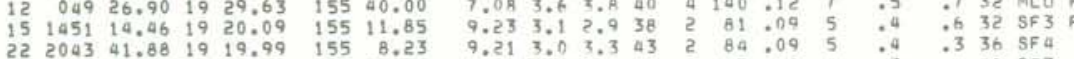

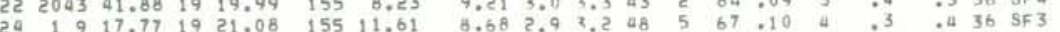

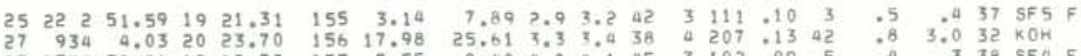

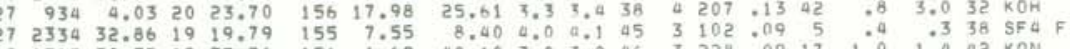

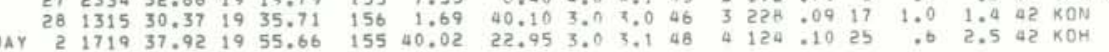

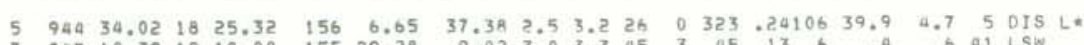

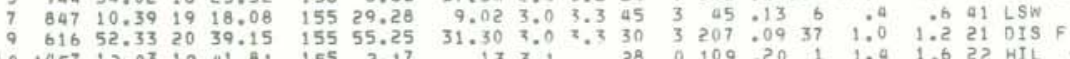

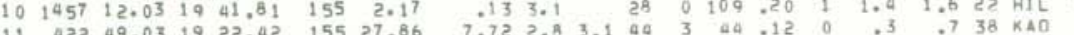

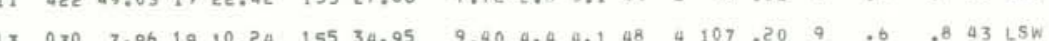

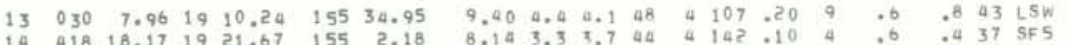

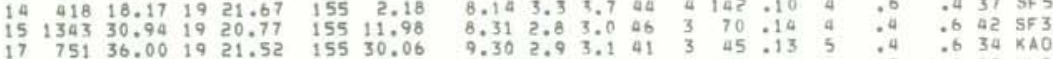

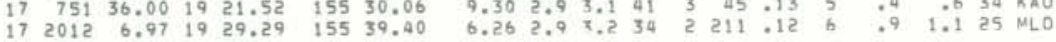

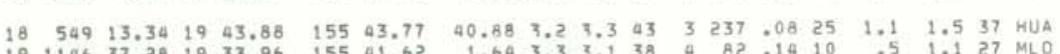

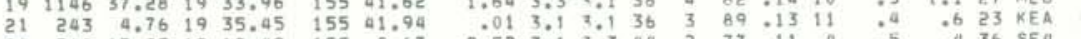

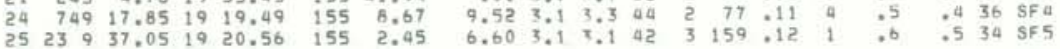

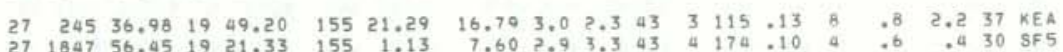

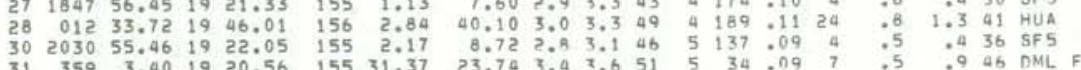

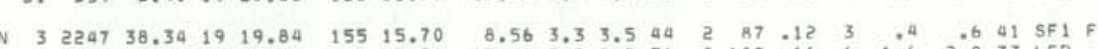

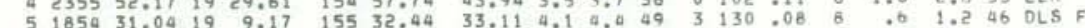

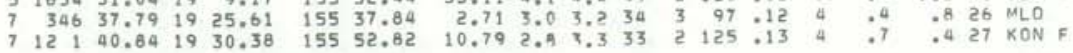

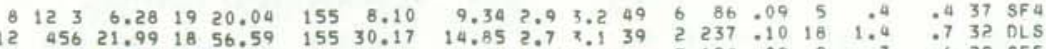

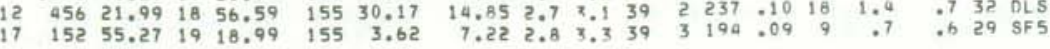


HVO EARTHQUAKE SUMMARY LIST

ORIGIN TIME LAT N
OA LON N

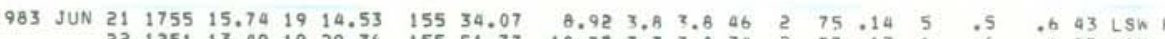

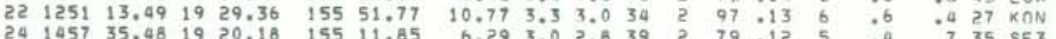

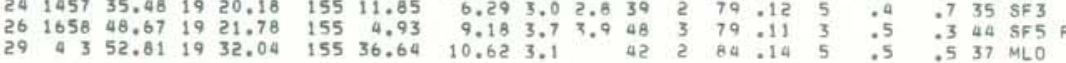

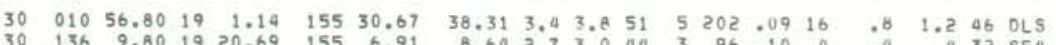

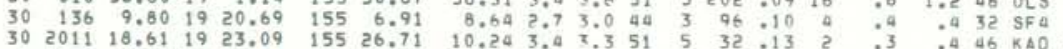

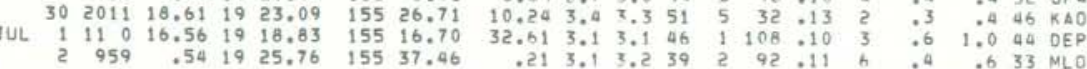

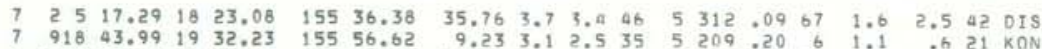

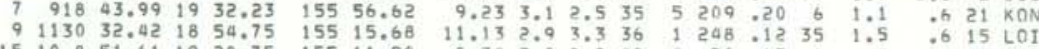

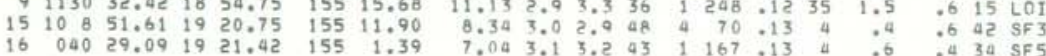

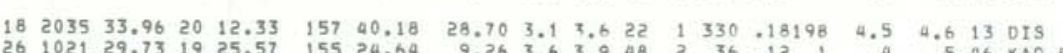

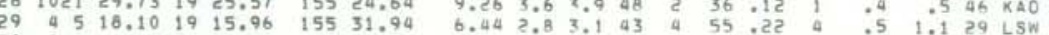

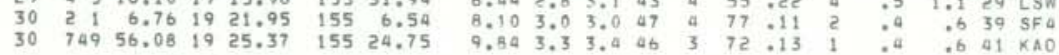

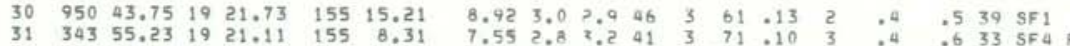

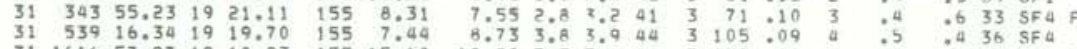

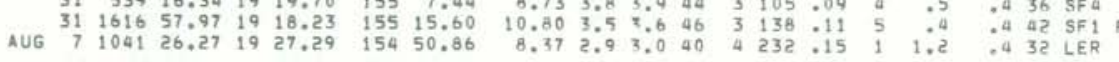

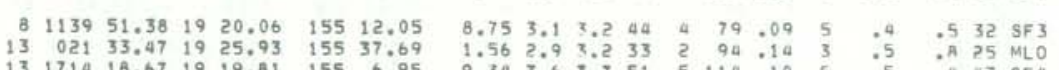

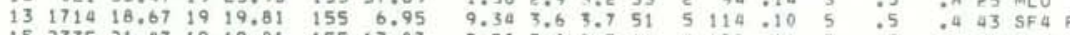

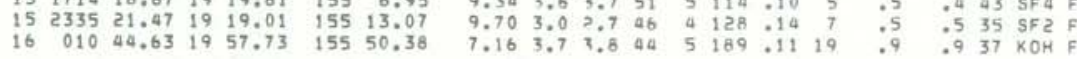

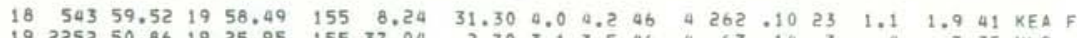

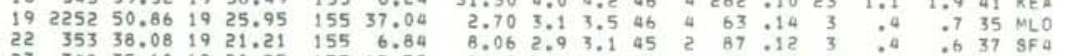

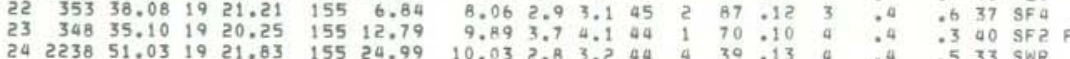

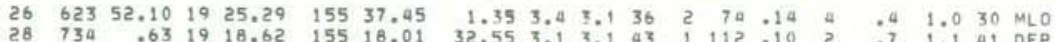

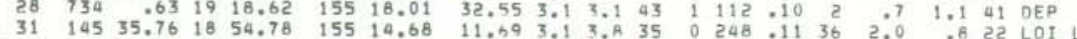

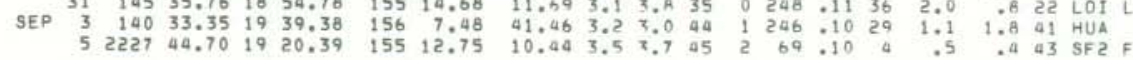

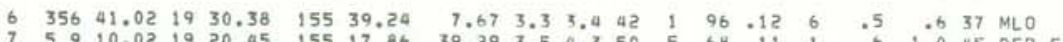

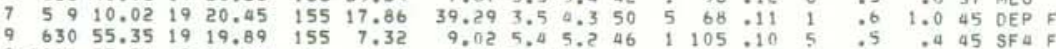

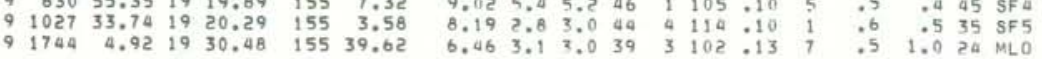

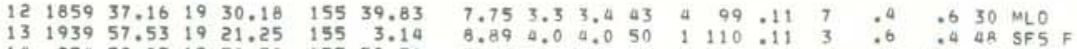

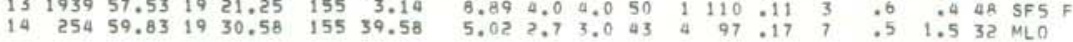

HVO EARTHQUAKE SUMMARY LIST

PAGE Q ORIGIN TIME LAT N LON W DEPTH AMP NUR GAP RMS MIN ERH ERZ NO

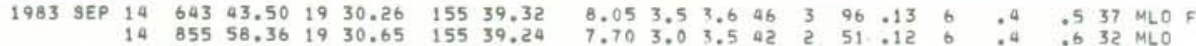

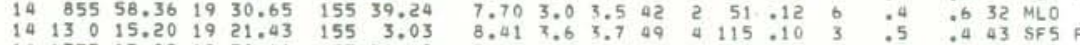

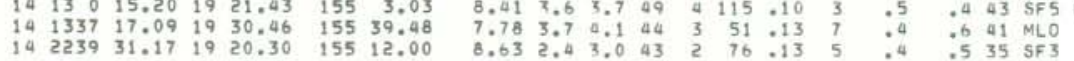

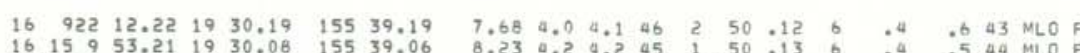

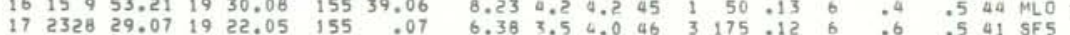

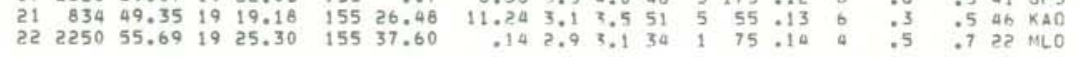

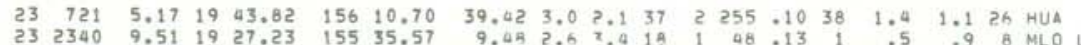

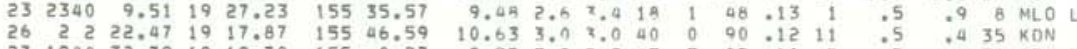

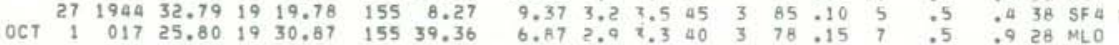

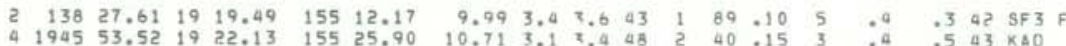

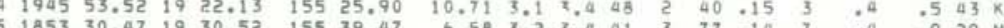

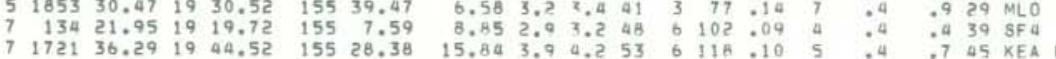

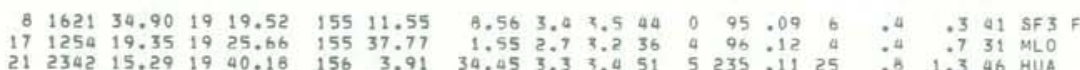

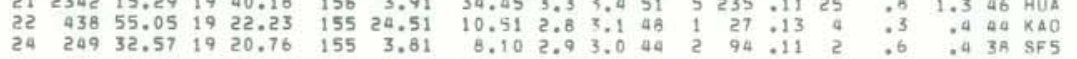

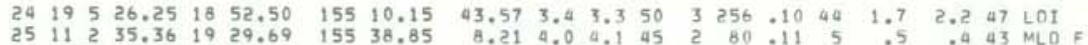

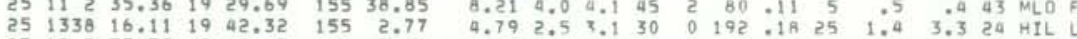

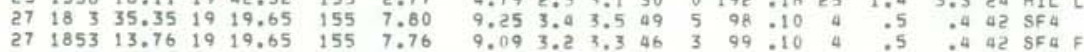

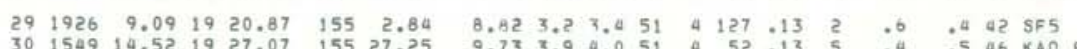

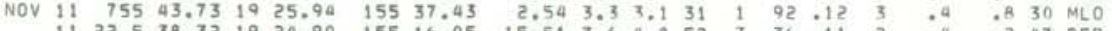

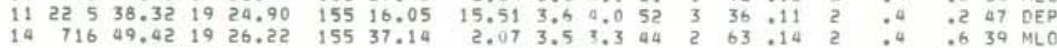

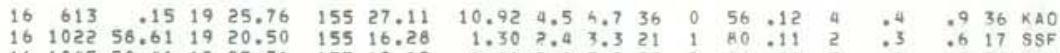

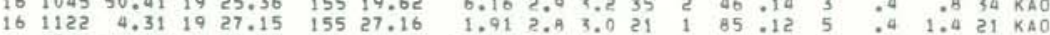

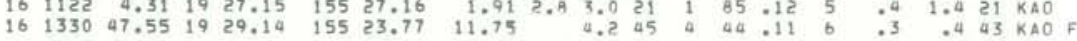

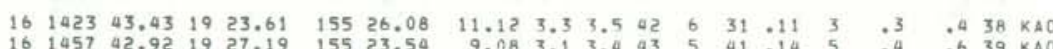

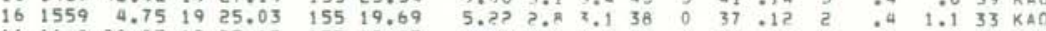

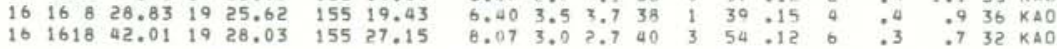

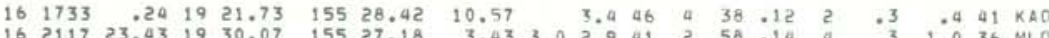

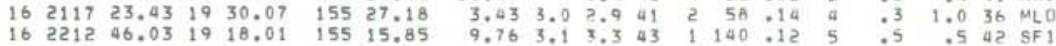


HVO EARTHQUAKE SUMMARY LIST

PAGE 5

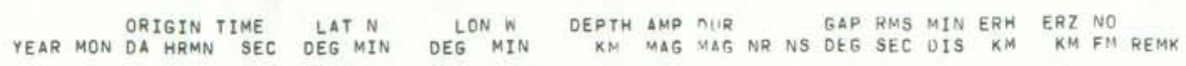
$\begin{array}{rlrlllllllllllllllll}1983 \text { NOV } & 17 & 417 & 8.64 & 19 & 21.57 & 155 & 19.63 & 31.97 & 2.7 & 7.1 & 47 & 3 & 45 & .12 & 4 & .6 & 1.0 & 44 & \text { DEP } \\ 17 & 1212 & 47.50 & 19 & 22.19 & 155 & 28.65 & 8.69 & 3.7 & 3.9 & 48 & 4 & 37 & .12 & 8 & .3 & .7 & 38 & K A O\end{array}$

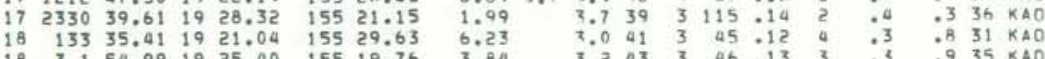
$\begin{array}{lllllllllllllllllll}18 & 651 & 4.78 & 19 & 24.95 & 155 & 21.92 & 11.04 & 2.5 & 3.3 & 49 & 6 & 36 & .11 & 5 & .3 & .4 & 38 & \text { KAO }\end{array}$

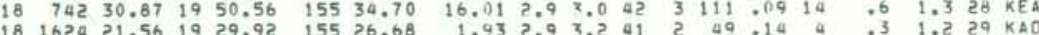

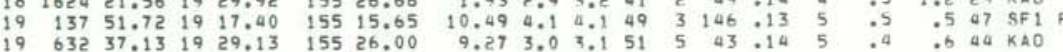

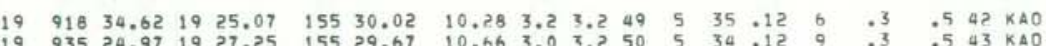

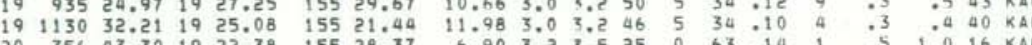

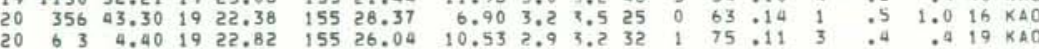

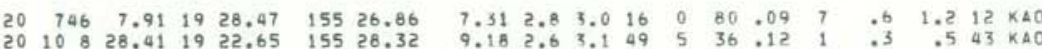
$\begin{array}{llllllll}50 & .543 k 40\end{array}$

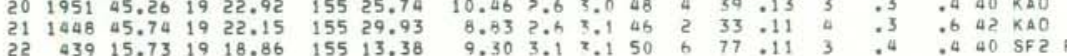

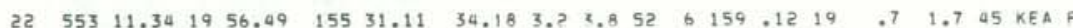

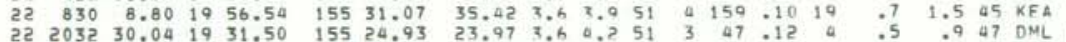

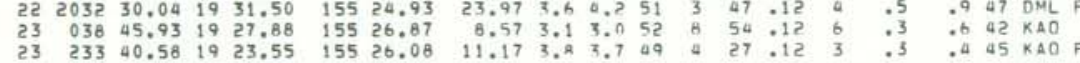

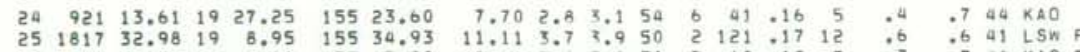

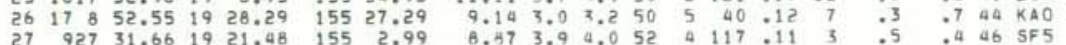

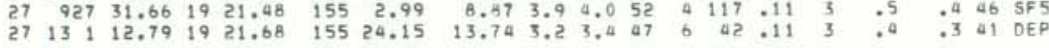

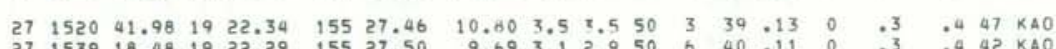

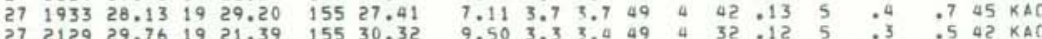

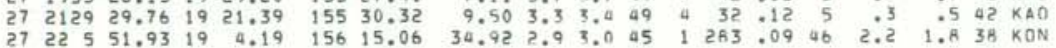

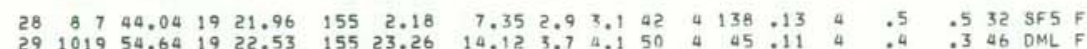

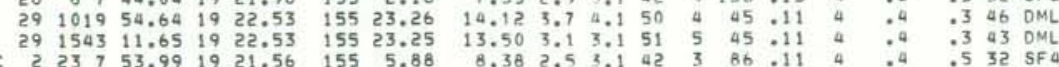

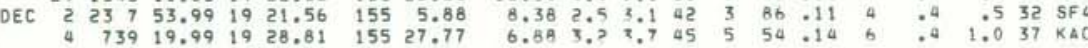

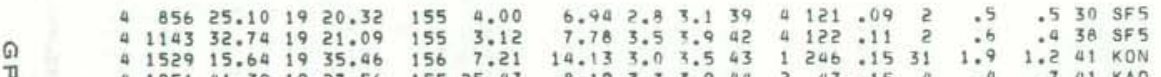

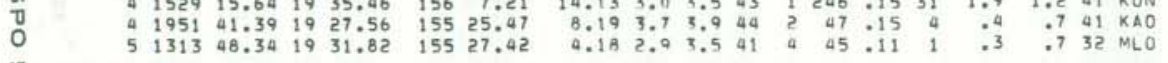

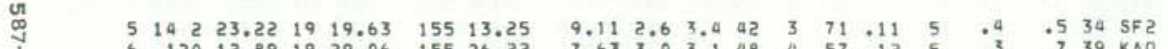

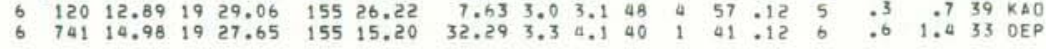

HVO EARTHQUAKE SUMMARY LIST

PAGE 6

ORIGIN TIME LAT N LON W OEPTH AMP RUR GAP RMS MIN ERH ERZ NO

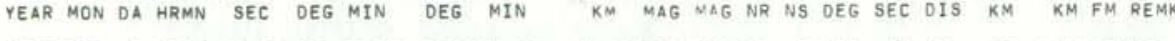
$\begin{array}{lllllllllllllllllllllll}1983 \text { DEC } & 6 & 1255 & 45.24 & 19 & 8.67 & 155 & 34.41 & 9.93 & 2.5 & 3.0 & 26 & 2 & 127 & .21 & 12 & .7 & 1.4 & 22 & \text { LSW } \\ & 7 & 345 & 11.37 & 19 & 22.29 & 155 & 29.73 & 9.30 & 2.9 & 3.2 & 45 & 2 & 33 & .10 & 4 & .3 & .7 & 42 & K A 0\end{array}$

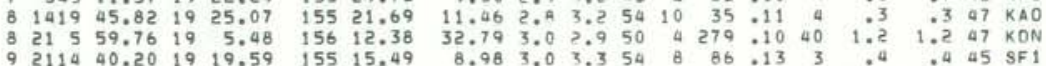

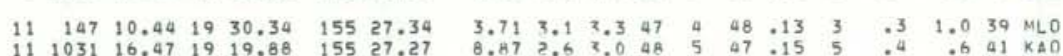

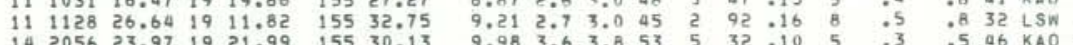

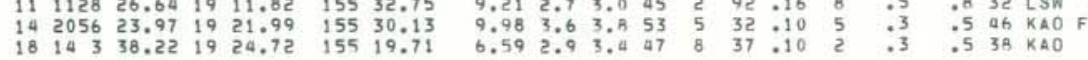
$\begin{array}{lllllllllllllllllll}18 & 2111 & 56.18 & 19 & 29.12 & 155 & 26.59 & 8.29 & 3.2 & 3.1 & 50 & 4 & 62 & .13 & 6 & .4 & .7 & 42 & \text { KAO } \\ 18 & 2341 & 31.74 & 19 & 21.50 & 155 & 30.08 & 9.62 & 3.3 & 3.6 & 51 & 6 & 33 & .11 & 5 & .3 & .5 & 43 & \text { KAO }\end{array}$

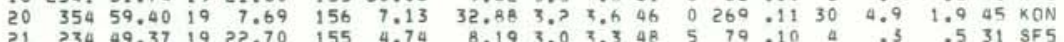

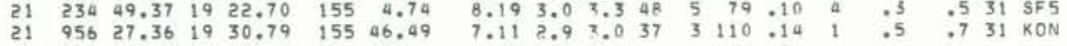

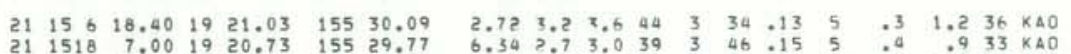

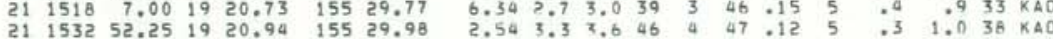

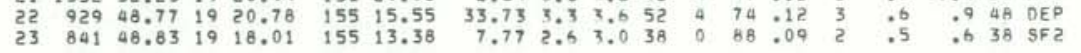

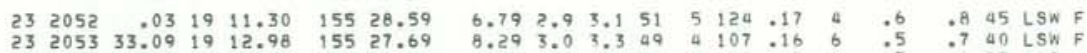

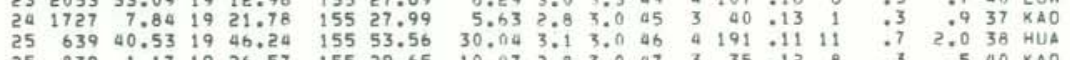

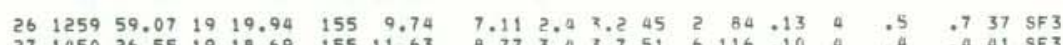

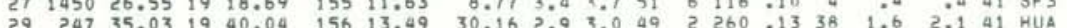

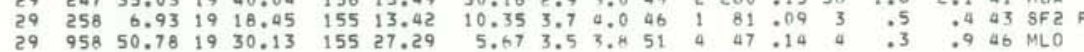

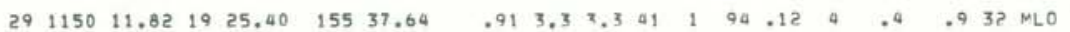

